\title{
Palladium-Catalyzed Homocoupling of Highly Fluorinated Arylboronates: Studies of the Influence of Strongly vs. Weakly Coordinating Solvents on the Reductive Elimination Process
}

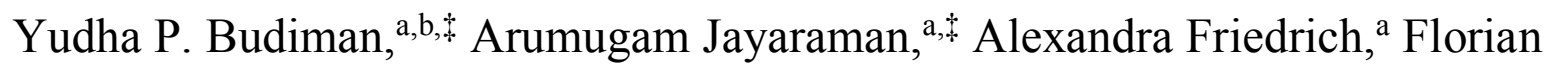
Kerner, ${ }^{\mathrm{a}}$ Udo Radius, ${ }^{\mathrm{a},}{ }^{*}$ Todd B. Marder ${ }^{\mathrm{a}, *}$

\footnotetext{
${ }^{a}$ Institut für Anorganische Chemie, and Institute for Sustainable Chemistry \& Catalysis with Boron, Julius-Maximilians-Universität Würzburg, Am Hubland, 97074 Würzburg, Germany

${ }^{b}$ Department of Chemistry, Faculty of Mathematics and Natural Sciences,

Universitas Padjadjaran, 45363 Jatinangor, Indonesia

†Y.P.B. and A.J. contributed equally.
}

Table of Contents

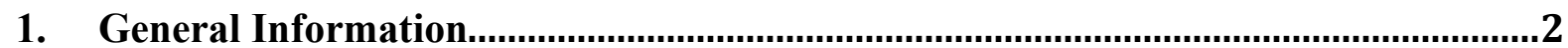

2. Synthesis and Characterization of Pentafluorophenyl Boronic Acid Pinacol Ester.3

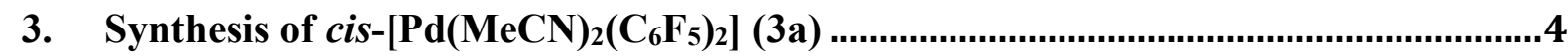

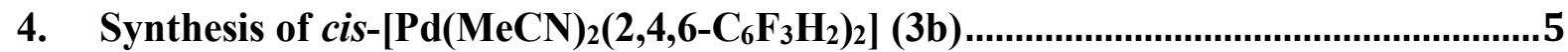

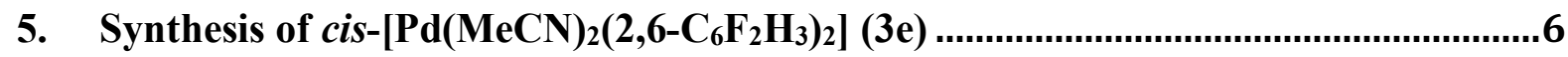

6. General Procedure for Homocoupling of Fluorinated Aryl Boronic Acid Pinacol

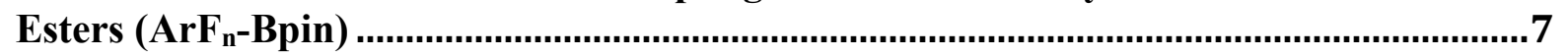

7. Experimental Procedures and Characterization of Products...................................8

8. A Test for the Possibility of C-F Oxidative Addition ............................................. 13

9. ${ }^{1} \mathrm{H},{ }^{13} \mathrm{C}\left\{{ }^{1} \mathrm{H}\right\},{ }^{11} \mathrm{~B}\left\{{ }^{1} \mathrm{H}\right\},{ }^{19} \mathrm{~F},{ }^{19} \mathrm{~F}\left\{{ }^{1} \mathrm{H}\right\}$ NMR, IR Spectra and GC-MS Data ................. 16

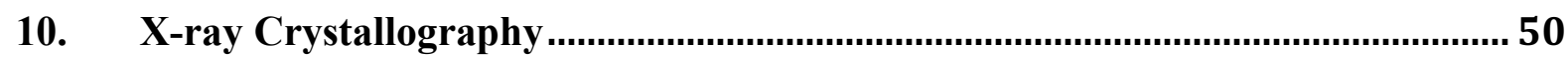

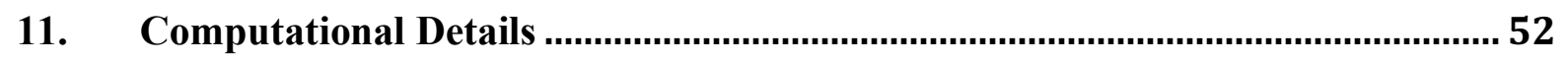

12. Optimized Structures, Cartesian Coordinates, and Energies ............................52

13. EDA-NOCV Analysis and Their Results .......................................................113

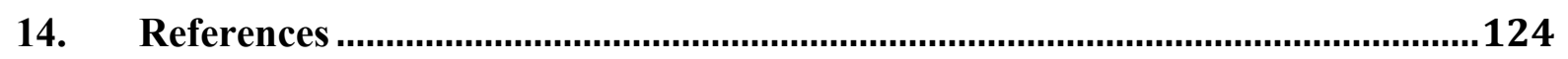




\section{General Information}

Unless otherwise noted, all reagents were purchased from Alfa-Aesar, Sigma-Aldrich, ABCR, VWR, Fluorochem or Acros, and were checked for purity by GC-MS and/or ${ }^{1} \mathrm{H}$ NMR spectroscopy and used as received. $\mathrm{B}_{2} \operatorname{pin}_{2}$ was kindly provided by AllyChem Co. Ltd. (Dalian, China). Acetonitrile (Honeywell, HPLC grade), triethylamine (Aldrich, $\geq 99.5 \%$ ), tetrahydrofuran (Carl Roth, HPLC grade), dimethyl sulfoxide (Acros, $\geq$ 99.8\%), dimethylformamide (VWR, HPLC grade), benzene (Honeywell, ACS Reagent grade), hexafluorobenzene (Fluorochem, $\geq 97 \%$ ), $m$-xylene (Acros, $\geq 99 \%$ ), and toluene (VWR, ACS Reagent grade) were used as received and were not dried.

Automated flash chromatography was performed using silica gel (Biotage SNAP cartridge KP-Sil $10 \mathrm{~g}$ and KP-Sil $25 \mathrm{~g}$ ) using a Biotage ${ }^{\circledR}$ Isolera Four system. Commercially available, precoated TLC plates (Polygram ${ }^{\circledR}$ Sil G/UV254) were purchased from MacheryNagel. The removal of solvent was performed on a rotary evaporator in vacuo at a maximum temperature of $30^{\circ} \mathrm{C}$.

GC-MS analyses were performed using an Agilent 7890A gas chromatograph (column: HP-5MS 5\% phenylmethylsiloxane, $10 \mathrm{~m}, \varnothing 0.25 \mathrm{~mm}$, film $0.25 \mu \mathrm{m}$; injector: 250 ${ }^{\circ} \mathrm{C}$; oven: $40{ }^{\circ} \mathrm{C}(2 \mathrm{~min}), 40{ }^{\circ} \mathrm{C}$ to $280{ }^{\circ} \mathrm{C}\left(20{ }^{\circ} \mathrm{C} \cdot \mathrm{min}^{-1}\right)$; carrier gas: He $\left(1.2 \mathrm{~mL} \mathrm{~min}{ }^{-1}\right)$ equipped with an Agilent 5975C inert MSD with triple-axis detector operating in EI mode and an Agilent 7693A series auto sampler/injector. HRMS analyses were performed using a Thermo Fischer Scientific Exactive Plus Orbitrap MS system (APCI and ASAP probe). Infrared spectra were recorded on a Nicolet 380 FT-IR spectrometer as solids, using an ATR unit, and are reported in $\mathrm{cm}^{-1}$. Elemental analyses were performed on a LECO CHNS-932 Elemental Analyzer in our institute.

All NMR spectra were recorded at $298 \mathrm{~K}$ using a Bruker Avance I $500\left({ }^{1} \mathrm{H}\right.$ NMR, 500 MHz; ${ }^{13} \mathrm{C}\left\{{ }^{1} \mathrm{H}\right\}$ NMR, $125 \mathrm{MHz} ;{ }^{13} \mathrm{C}\left\{{ }^{19} \mathrm{~F}\right\} \mathrm{NMR}, 125 \mathrm{MHz} ;{ }^{19} \mathrm{~F}$ NMR, $470 \mathrm{MHz}$ ), a Bruker 
DRX-300 $\left({ }^{1} \mathrm{H}, 300 \mathrm{MHz} ;{ }^{11} \mathrm{~B}\left\{{ }^{1} \mathrm{H}\right\}, 96 \mathrm{MHz}\right)$, or a Bruker Avance Neo $\left({ }^{13} \mathrm{C}\left\{{ }^{1} \mathrm{H}\right\} \mathrm{NMR}, 101\right.$ $\left.\mathrm{MHz} ;{ }^{19} \mathrm{~F}\left\{{ }^{1} \mathrm{H}\right\} \mathrm{NMR}, 376 \mathrm{MHz}\right)$ spectrometer. ${ }^{1} \mathrm{H}$ NMR chemical shifts are reported relative to TMS and were referenced via residual proton resonances of the corresponding deuterated solvent $\left(\mathrm{CDCl}_{3}: 7.26\right.$ ppm; $\mathrm{C}_{6} \mathrm{D}_{6}: 7.16$ ppm; $\mathrm{CD}_{3} \mathrm{CN}: 1.94$; DMSO- $\left.d_{6}: 2.50 \mathrm{ppm}\right),{ }^{13} \mathrm{C}\left\{{ }^{1} \mathrm{H}\right\}$ NMR and ${ }^{13} \mathrm{C}\left\{{ }^{19} \mathrm{~F}\right\}$ NMR spectra are reported relative to TMS via the carbon signals of the deuterated solvent $\left(\mathrm{CDCl}_{3}: 77.16\right.$ ppm; $\mathrm{C}_{6} \mathrm{D}_{6}: 128.06$ ppm; $\mathrm{CD}_{3} \mathrm{CN}: 1.32 \mathrm{ppm}, 118.26 \mathrm{ppm}$; DMSO- $\left.d_{6}: 39.52 \mathrm{ppm}\right),{ }^{19} \mathrm{~F}$ NMR and ${ }^{19} \mathrm{~F}\left\{{ }^{1} \mathrm{H}\right\}$ spectra are reported relative to external $\mathrm{CFCl}_{3}$, and ${ }^{11} \mathrm{~B}\left\{{ }^{1} \mathrm{H}\right\}$ NMR chemical shifts are quoted relative to $\mathrm{BF}_{3} \cdot \mathrm{Et}_{2} \mathrm{O}$ as external standard.

\section{Synthesis and Characterization of Pentafluorophenyl Boronic Acid Pinacol Ester ${ }^{\mathrm{S} 1}$}
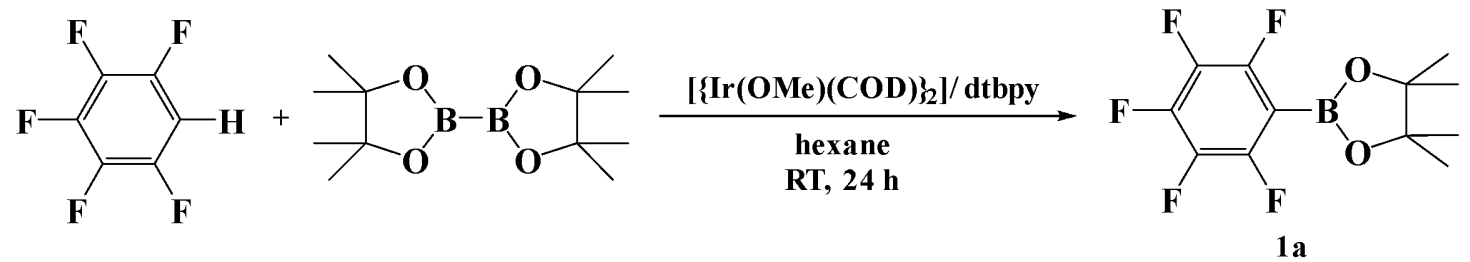

$\mathrm{C}_{6} \mathrm{~F}_{5} \mathrm{Bpin}(\mathbf{1 a})$ was synthesized via $\mathrm{C}-\mathrm{H}$ borylation according to a literature procedure. ${ }^{\mathrm{S} 1} \mathrm{In}$ a glove box, bis(pinacolato)diboron $(9.06 \mathrm{~g}, 35.70 \mathrm{mmol}),[\operatorname{Ir}(\mathrm{COD})(\mathrm{OMe})]_{2}(0.47 \mathrm{~g}, 0.71$ mmol), 4-4' -di-tert-butyl-2,2' -bypyridine ( $0.38 \mathrm{~g} / 1.42 \mathrm{mmol})$, degassed pentafluorobenzene $(6.00 \mathrm{~g}, 35.70 \mathrm{mmol})$, and $50 \mathrm{~mL}$ of hexane was added into a $250 \mathrm{~mL}$ two-neck round bottom flask. The flask was capped, and the solution was stirred at room temperature. After $24 \mathrm{~h}$, the solution was concentrated in vacuo. The residue was purified via column chromatography on silica gel (hexane). After concentrating the fractions containing the product, the residue was dried under reduced pressure to yield the pure corresponding product as a white solid ( $9.95 \mathrm{~g}$, $95 \%)$.

${ }^{\mathbf{1}} \mathbf{H}$ NMR $\left(300 \mathrm{MHz}, \mathrm{CDCl}_{3}\right) \delta=1.38\left(s, 12 \mathrm{H}, \mathrm{CH}_{3}\right) ;{ }^{\mathbf{1}} \mathbf{C}\left\{{ }^{\mathbf{1}} \mathbf{H}\right\}$ NMR $\left(125 \mathrm{MHz}, \mathrm{CDCl}_{3}\right) \delta=$ $149.4\left(d m,{ }^{1} J_{\mathrm{F}-\mathrm{C}}=251 \mathrm{~Hz}, \mathrm{C}_{\mathrm{arom}}-\mathrm{F}_{\mathrm{o}}\right), 143.1\left(d m,{ }^{1} J_{\mathrm{F}-\mathrm{C}}=257 \mathrm{~Hz}, \mathrm{C}_{\mathrm{arom}}-\mathrm{F}_{\mathrm{p}}\right), 137.4\left(d m,{ }^{1} J_{\mathrm{F}-\mathrm{C}}=\right.$ $\left.252 \mathrm{~Hz}, \mathrm{C}_{\text {arom }}-\mathrm{F}_{\mathrm{m}}\right), 85.2,24.8\left(\mathrm{CH}_{3}\right) ;{ }^{19} \mathbf{F} \mathbf{N M R}\left(470 \mathrm{MHz}, \mathrm{CDCl}_{3}\right) \delta=-162.0--161.9(m$, 
$\left.2 \mathrm{~F}_{\mathrm{o}}\right),-149.8\left(t t,{ }^{3} J_{\mathrm{F}-\mathrm{F}}=20 \mathrm{~Hz},{ }^{4} J_{\mathrm{F}-\mathrm{F}}=4 \mathrm{~Hz}, 1 \mathrm{~F}_{\mathrm{p}}\right),-129.6--129.5\left(m, 2 \mathrm{~F}_{\mathrm{m}}\right) .{ }^{11} \mathbf{B}\left\{{ }^{1} \mathbf{H}\right\} \mathrm{NMR}(96$ $\left.\mathrm{MHz}, \mathrm{CDCl}_{3}\right) \delta=29.0$. GC-MS: $[\mathrm{t}=8.058 \mathrm{~min}] \mathrm{m} / \mathrm{z}: 294[M]^{+}$. HRMS (ASAP) calcd. for $\left[\mathrm{C}_{12} \mathrm{H}_{13} \mathrm{BF}_{5} \mathrm{O}_{2}\right]^{+}: 295.0923[M+\mathrm{H}]^{+}$; found: 295.0912. Spectroscopic data matched those in the literature. ${ }^{\mathrm{S} 2}$

\section{Synthesis of cis-[Pd(MeCN) $\left.)_{2}\left(\mathrm{C}_{6} \mathrm{~F}_{5}\right)_{2}\right](3 \mathrm{a})$}

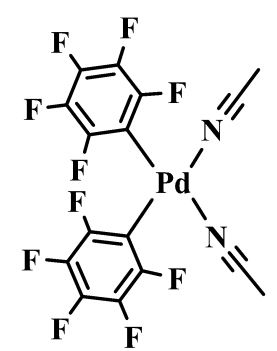

In air, into a dried vial containing $4 \mathrm{~mL}$ of $\mathrm{MeCN}$ solvent and equipped with a stirring bar was added $\mathrm{Pd}(\mathrm{OAc})_{2}(90 \mathrm{mg}, 0.40 \mathrm{mmol})$ and the mixture was stirred until homogenous. Then, $\mathrm{C}_{6} \mathrm{~F}_{5} \mathrm{Bpin}(\mathbf{1 a})(353 \mathrm{mg}, 1.2 \mathrm{mmol})$ or $\mathrm{C}_{6} \mathrm{~F}_{5} \mathrm{H}(672 \mathrm{mg}, 4 \mathrm{mmol})$ and $\mathrm{Ag}_{2} \mathrm{O}(371 \mathrm{mg}$, $1.60 \mathrm{mmol})$ were added to the solution and the vial was sealed. The suspension was then stirred at $60{ }^{\circ} \mathrm{C}$ for $16 \mathrm{~h}$. After cooling to room temperature, the resulting mixture was filtered through a pad of Celite. The solution was evaporated to dryness under reduced pressure at $30{ }^{\circ} \mathrm{C}$ and the residue was diluted with $\mathrm{Et}_{2} \mathrm{O}: \mathrm{DCM}(1: 1)$. The resulting precipitate was collected by filtration and washed with $\mathrm{Et}_{2} \mathrm{O}: \mathrm{DCM}(1: 1)$, hexane, and air-dried to give the product as a brownish-white solid (157 mg, 75\%, if via $\left.\mathrm{C}_{6} \mathrm{~F}_{5} \mathrm{Bpin}\right),(157 \mathrm{mg}, 74 \%$, if via $\left.\mathrm{C}_{6} \mathrm{~F}_{5} \mathrm{H}\right)$. Colorless single crystals suitable for $\mathrm{X}$-ray diffraction were grown via vapor diffusion of $\mathrm{CD}_{3} \mathrm{CN} / \mathrm{Et}_{2} \mathrm{O}$ solutions placed in a larger vessel containing toluene at room temperature.

${ }^{1} \mathbf{H}$ NMR $\left(500 \mathrm{MHz}, \mathrm{DMSO}-d_{6}\right) \delta=2.07\left(s, 6 \mathrm{H}, \mathrm{CH}_{3} \mathrm{CN}\right) ;{ }^{13} \mathbf{C}\left\{{ }^{1} \mathbf{H}\right\}$ NMR $(125 \mathrm{MHz}$, DMSO- $\left.d_{6}\right) \delta=146.9\left(d m,{ }^{1} J_{\mathrm{H}-\mathrm{C}}=226 \mathrm{~Hz}, \mathrm{C}_{\mathrm{arom}}-\mathrm{F}_{\mathrm{o}}\right), 136.8\left(d m,{ }^{1} J_{\mathrm{H}-\mathrm{C}}=212 \mathrm{~Hz}, \mathrm{C}_{\text {arom }}-\mathrm{F}_{\mathrm{p}}\right)$, $135.0\left(d m,{ }^{1} J_{\mathrm{H}-\mathrm{C}}=240 \mathrm{~Hz}, \mathrm{C}_{\text {arom }}-\mathrm{F}_{\mathrm{m}}\right), 118.1\left(\mathrm{CH}_{3} \mathrm{CN}\right), 1.2\left(\mathrm{CH}_{3} \mathrm{CN}\right) ;{ }^{13} \mathbf{C}\left\{{ }^{\mathbf{1}} \mathbf{F}\right\} \mathbf{N M R}(125$ MHz, DMSO- $d_{6}$, for $\left.\mathbf{C}_{6} \mathbf{F}_{5}\right) \delta=146.9\left(\mathrm{C}_{\text {arom }}-\mathrm{F}_{\mathrm{o}}\right), 136.8\left(\mathrm{C}_{\text {arom }}-\mathrm{F}_{\mathrm{p}}\right), 135.0\left(\mathrm{C}_{\text {arom }}-\mathrm{F}_{\mathrm{m}}\right) 118.1(m$, 
$\left.\mathrm{CH}_{3} \mathrm{CN}\right), 1.2\left(q,{ }^{1} \mathrm{~J}_{\mathrm{H}-\mathrm{C}}=136 \mathrm{~Hz}, \mathrm{CH}_{3} \mathrm{CN}\right)$; the chemical shift of $\mathrm{C}_{\text {arom }}-\mathrm{Pd}$ can be seen clearly in $\mathrm{CD}_{3} \mathrm{CN} ;{ }^{13} \mathbf{C}\left\{{ }^{19} \mathbf{F}\right\}$ NMR $\left(125 \mathrm{MHz}, \mathrm{CD}_{3} \mathrm{CN}\right.$, for $\left.\mathbf{C}_{\mathbf{6}} \mathbf{F}_{\mathbf{5}}\right) \delta=148.4\left(\mathrm{C}_{\text {arom }}-\mathrm{F}_{\mathrm{o}}\right), 138.3\left(\mathrm{C}_{\text {arom }}{ }^{-}\right.$ $\left.\mathrm{F}_{\mathrm{p}}\right), 136.6\left(\mathrm{C}_{\text {arom }}-\mathrm{F}_{\mathrm{m}}\right), 109.4\left(\mathrm{C}_{\text {arom }}-\mathrm{Pd}\right)$, the chemical shifts of $\mathrm{CH}_{3} \mathrm{CN}$ overlapped with those of $\mathrm{CD}_{3} \mathrm{CN} ;{ }^{19} \mathbf{F}\left\{{ }^{1} \mathbf{H}\right\}$ NMR $\left(376 \mathrm{MHz}, \mathrm{CD}_{3} \mathrm{CN}\right) \delta=-166.1--165.9\left(m, 4 \mathrm{~F}_{\mathrm{m}}\right),-162.9\left(t,{ }^{3} J_{\mathrm{F}-\mathrm{F}}\right.$ $\left.=19 \mathrm{~Hz}, 2 \mathrm{~F}_{\mathrm{p}}\right),-117.7--117.6\left(m, 4 \mathrm{~F}_{\mathrm{o}}\right) ; \mathrm{Mp}: 163{ }^{\circ} \mathrm{C}$ dec.; Anal. Calcd for $\mathrm{C}_{16} \mathrm{H}_{6} \mathrm{~F}_{10} \mathrm{~N}_{2} \mathrm{Pd}: \mathrm{C}$, 36.77; H, 1.16; N, 5.36. Found: C, 36.93; H, 1.44; N, 5.59; IR $\left(\right.$ ATR $\left.\left[\mathrm{cm}^{-1}\right]\right)=2331\left(v_{\mathrm{CN}}\right)$, $2304\left(v_{\mathrm{CN}}\right)$.

\section{Synthesis of cis-[Pd( $\left.\mathrm{MeCN})_{2}\left(2,4,6-\mathrm{C}_{6} \mathrm{~F}_{3} \mathrm{H}_{2}\right)_{2}\right](3 \mathrm{~b})$}

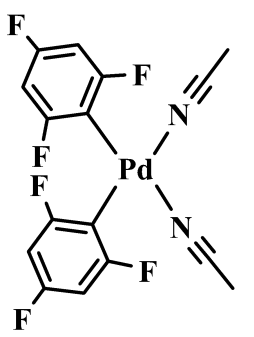

In air, into a dried vial containing $4 \mathrm{~mL}$ of $\mathrm{MeCN}$ solvent and equipped with a stirring bar was added $\mathrm{Pd}(\mathrm{OAc})_{2}(90 \mathrm{mg}, 0.40 \mathrm{mmol})$ and the mixture was stirred until homogeneous. Then, 2,4,6-trifluorophenyl-Bpin (1b) (310 mg, $1.20 \mathrm{mmol})$ and $\mathrm{Ag}_{2} \mathrm{O}$ (371 mg, $\left.1.60 \mathrm{mmol}\right)$ were added to the solution and the vial was sealed. The suspension was then stirred at $60{ }^{\circ} \mathrm{C}$ for $16 \mathrm{~h}$. After cooling to room temperature, the resulting mixture was filtered through a pad of Celite. The solution was evaporated to dryness under reduced pressure at $30{ }^{\circ} \mathrm{C}$ and the residue was diluted with $\mathrm{Et}_{2} \mathrm{O}: \mathrm{DCM}(1: 1)$ and the resulting precipitate was collected via filtration to give a pale yellow solution. This was concentrated, and addition of hexane precipitated a yellow solid, which was collected by filtration, washed with hexane, and airdried to give a pale yellow solid (144 mg, 80\%). Colorless single crystals suitable for X-ray diffraction were grown via vapor diffusion of $\mathrm{Et}_{2} \mathrm{O}$ solutions placed in a larger vessel containing hexane at room temperature. 
${ }^{1} \mathbf{H}$ NMR $\left(300 \mathrm{MHz}, \mathrm{CDCl}_{3}\right) \delta=6.31\left(q,{ }^{3} J_{\mathrm{H}-\mathrm{F}}=5 \mathrm{~Hz}, 2 \mathrm{H}, \mathrm{C}_{\text {arom }}-\mathrm{H}\right), 2.14\left(s, 6 \mathrm{H}, \mathrm{CH}_{3} \mathrm{CN}\right)$; ${ }^{13} \mathbf{C}\left\{{ }^{1} \mathbf{H}\right\}$ NMR $\left(125 \mathrm{MHz}, \mathrm{DMSO}-d_{6}\right) \delta=165.2\left(d m,{ }^{1} J_{\mathrm{F}-\mathrm{C}}=230.8 \mathrm{~Hz}, \mathrm{C}_{\text {arom }}-\mathrm{F}_{\mathrm{o}}\right), 160.6(d m$, $\left.{ }^{1} J_{\mathrm{F}-\mathrm{C}}=237.8 \mathrm{~Hz}, \mathrm{C}_{\text {arom }}-\mathrm{F}_{\mathrm{p}}\right) 118.1\left(\mathrm{~m}, \mathrm{CH}_{3} \mathrm{CN}\right), 98.4\left(d d,{ }^{2} J_{\mathrm{F}-\mathrm{C}}=38 \mathrm{~Hz},{ }^{2} J_{\mathrm{F}-\mathrm{C}}=24 \mathrm{~Hz}, \mathrm{C}_{\text {arom }}{ }^{-}\right.$ $\mathrm{H}), 1.2\left(\mathrm{CH}_{3} \mathrm{CN}\right) ;{ }^{13} \mathbf{C}\left\{{ }^{\mathbf{1 9}} \mathbf{F}\right\}$ NMR $\left(125 \mathrm{MHz}, \mathrm{DMSO}-d_{6}\right) \delta=165.2\left(m, \mathrm{C}_{\text {arom }}-\mathrm{F}_{\mathrm{o}}\right), 160.5(t$, $\left.{ }^{2} J_{\mathrm{H}-\mathrm{C}}=6 \mathrm{~Hz}, \mathrm{C}_{\mathrm{arom}}-\mathrm{F}_{\mathrm{p}}\right), 118.1\left(m, \mathrm{CH}_{3} C \mathrm{~N}\right), 98.4\left(d,{ }^{1} J_{\mathrm{H}-\mathrm{C}}=166 \mathrm{~Hz},{ }^{3} J_{\mathrm{H}-\mathrm{C}}=4 \mathrm{~Hz}, \mathrm{C}_{\text {arom }}-\mathrm{H}\right)$, $1.1\left(q,{ }^{1} J_{\mathrm{H}-\mathrm{C}}=136 \mathrm{~Hz}, \mathrm{CH}_{3} \mathrm{CN}\right)$; the chemical shift of $\mathrm{C}_{\text {arom- }} \mathrm{Pd}$ can be seen clearly in $\mathrm{CDCl}_{3}$; ${ }^{13} \mathbf{C}\left\{{ }^{19} \mathbf{F}\right\}$ NMR $\left(125 \mathrm{MHz}, \mathrm{CDCl}_{3}\right) \delta=165.9\left(m, \mathrm{C}_{\mathrm{arom}}-\mathrm{F}_{\mathrm{o}}\right), 161.1\left(t,{ }^{2} J_{\mathrm{H}-\mathrm{C}}=6 \mathrm{~Hz}, \mathrm{C}_{\mathrm{arom}}-\mathrm{F}_{\mathrm{p}}\right)$, $117.4\left(m, \mathrm{CH}_{3} \mathrm{CN}\right), 106.3\left(\mathrm{C}_{\mathrm{arom}}-\mathrm{Pd}\right), 98.2\left(d d,{ }^{1} J_{\mathrm{H}-\mathrm{C}}=165 \mathrm{~Hz},{ }^{3} J_{\mathrm{H}-\mathrm{C}}=4 \mathrm{~Hz}, \mathrm{C}_{\mathrm{arom}}-\mathrm{H}\right), 2.9(q$, $\left.{ }^{1} J_{\mathrm{H}-\mathrm{C}}=146 \mathrm{~Hz}, C \mathrm{H}_{3} \mathrm{CN}\right) ;{ }^{19} \mathbf{F}\left\{{ }^{1} \mathbf{H}\right\}$ NMR $\left(376 \mathrm{MHz}, \mathrm{CD}_{3} \mathrm{CN}\right) \delta=-119.8--119.7\left(m, 2 \mathrm{~F}_{\mathrm{p}}\right),-$ $86.3--86.2\left(m,{ }^{4} J_{\mathrm{F}-\mathrm{F}}=3 \mathrm{~Hz}, 4 \mathrm{~F}_{\mathrm{o}}\right) ; \mathrm{Mp}: 133{ }^{\circ} \mathrm{C}$ dec.; Anal. Calcd for $\mathrm{C}_{16} \mathrm{H}_{10} \mathrm{~F}_{6} \mathrm{~N}_{2} \mathrm{Pd}: \mathrm{C}$, 42.64; H, 2.24; N, 6.22. Found: C, 42.25; H, 2.22; N, 6.22; IR (ATR [ $\left.\left.\mathrm{cm}^{-1}\right]\right): 2324\left(v_{\mathrm{CN}}\right)$, $2230\left(v_{\mathrm{CN}}\right)$.

\section{Synthesis of cis- $\left[\mathrm{Pd}(\mathrm{MeCN})_{2}\left(2,6-\mathrm{C}_{6} \mathrm{~F}_{2} \mathrm{H}_{3}\right)_{2}\right](3 \mathrm{e})$}

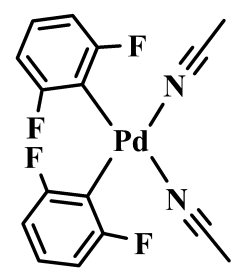

In air, into a dried vial containing $4 \mathrm{~mL}$ of $\mathrm{MeCN}$ solvent and equipped with a stirring bar was added $\mathrm{Pd}(\mathrm{OAc})_{2}(90 \mathrm{mg}, 0.40 \mathrm{mmol})$ and the mixture was stirred until homogenous. Then, 2,6-difluorophenylboronic acid pinacol ester (1g) (288 mg, $1.20 \mathrm{mmol})$, and $\operatorname{Ag}_{2} \mathrm{O}$ (371 mg, $1.60 \mathrm{mmol}$ ) were added to the solution and the vial was sealed. The suspension was then stirred at $60{ }^{\circ} \mathrm{C}$ for $16 \mathrm{~h}$. After cooling to room temperature, the resulting mixture was filtered through a pad of Celite. The solution was evaporated to dryness under reduced pressure at $30^{\circ} \mathrm{C}$ and the residue was diluted with $\mathrm{Et}_{2} \mathrm{O}: \mathrm{DCM}(1: 1)$. The resulting precipitate was collected by filtration and washed with $\mathrm{Et}_{2} \mathrm{O}: \mathrm{DCM}(1: 1)$, hexane, and air dried to give 
the product as a yellowish-white solid (155 mg, 93\%). Colorless single crystals suitable for $\mathrm{X}$-ray diffraction were grown via vapor diffusion of $\mathrm{CD}_{3} \mathrm{CN} / \mathrm{Et}_{2} \mathrm{O}$ solutions placed in a larger vessel containing toluene at room temperature.

${ }^{1} \mathbf{H}$ NMR $\left(300 \mathrm{MHz}, \mathrm{DMSO}-d_{6}\right) \delta=6.96-6.86\left(m, 2 \mathrm{H}, \mathrm{C}_{\mathrm{arom}}-\mathrm{H}_{\mathrm{p}}\right), 6.57-6.53\left(d d,{ }^{3} J_{\mathrm{H}-\mathrm{H}}=8\right.$ $\left.\mathrm{Hz},{ }^{3} J_{\mathrm{H}-\mathrm{F}}=6 \mathrm{~Hz}, 4 \mathrm{H}, \mathrm{C}_{\text {arom }}-\mathrm{H}_{\mathrm{m}}\right), 2.07\left(s, 6 \mathrm{H}, \mathrm{CH}_{3} \mathrm{CN}\right) ;{ }^{13} \mathbf{C}\left\{{ }^{1} \mathbf{H}\right\}$ NMR $\left(101 \mathrm{MHz}, \mathrm{DMSO}-d_{6}\right)$ $\delta=126.2\left(\mathrm{C}_{\text {arom }}-\mathrm{H}_{\mathrm{p}}\right), 118.1\left(\mathrm{CH}_{3} \mathrm{CN}\right), 1.1\left(\mathrm{CH}_{3} \mathrm{CN}\right)$; due to low solubility in DMSO- $d_{6}$, the chemical shifts of $\mathrm{C}_{\text {arom }}-\mathrm{F}$ and $\mathrm{C}_{\text {arom }}-\mathrm{Pd}$ bonds were not detected, but it can been seen clearly in $\mathrm{CD}_{3} \mathrm{CN} ;{ }^{13} \mathbf{C}\left\{{ }^{1} \mathbf{H}\right\}$ NMR $\left(101 \mathrm{MHz}, \mathrm{CD}_{3} \mathrm{CN}\right) \delta=167.8\left(d m,{ }^{1} J_{\mathrm{F}-\mathrm{C}}=231 \mathrm{~Hz}, \mathrm{C}_{\text {arom-m}} \mathrm{F}\right), 126.5$ $\left(m, \mathrm{C}_{\text {arom }}-\mathrm{H}_{\mathrm{p}}\right), 114.6\left(\mathrm{C}_{\mathrm{arom}}-\mathrm{Pd}\right), 110.0\left(d,{ }^{2} J_{\mathrm{F}-\mathrm{C}}=33 \mathrm{~Hz}, \mathrm{C}_{\mathrm{arom}}-\mathrm{H}_{\mathrm{m}}\right)$, the chemical shifts of $\mathrm{CH}_{3} \mathrm{CN}$ overlapped with those of $\mathrm{CD}_{3} \mathrm{CN} ;{ }^{13} \mathbf{C}\left\{{ }^{19} \mathbf{F}\right\} \mathbf{N M R}\left(126 \mathrm{MHz}, \mathrm{CD}_{3} \mathrm{CN}\right) \delta=167.7$ $\left(d m,{ }^{2} J_{\mathrm{H}-\mathrm{C}}=13 \mathrm{~Hz}, \mathrm{C}_{\text {arom }}-\mathrm{F}\right), 126.5\left(d,{ }^{1} J_{\mathrm{H}-\mathrm{C}}=162 \mathrm{~Hz}, \mathrm{C}_{\text {arom }}-\mathrm{H}_{\mathrm{p}}\right), 114.6\left(\mathrm{C}_{\text {arom}}-\mathrm{Pd}\right), 110.0$ $\left(d d,{ }^{1} J_{\mathrm{H}-\mathrm{C}}=161 \mathrm{~Hz},{ }^{2} J_{\mathrm{H}-\mathrm{C}}=9 \mathrm{~Hz}, \mathrm{C}_{\mathrm{arom}}-\mathrm{H}_{\mathrm{m}}\right)$, the chemical shifts of $\mathrm{CH}_{3} \mathrm{CN}$ overlapped with those of $\mathrm{CD}_{3} \mathrm{CN} ;{ }^{19} \mathbf{F}\left\{{ }^{1} \mathbf{H}\right\}$ NMR $\left(376 \mathrm{MHz}, \mathrm{CD}_{3} \mathrm{CN}\right) \delta=-88.5\left(s, 4 \mathrm{~F}_{\mathrm{o}}\right) ; \mathbf{M p}: 129{ }^{\circ} \mathrm{C}$ dec.; Anal. Calcd for $\mathrm{C}_{16} \mathrm{H}_{12} \mathrm{~F}_{4} \mathrm{~N}_{2} \mathrm{Pd}$ : C, 46.34; H, 2.92; N, 6.76. Found: C, 46.02; H, 2.89; N, 6.62. IR (ATR $\left.\left[\mathrm{cm}^{-1}\right]\right): 2322\left(v_{\mathrm{CN}}\right), 2294\left(v_{\mathrm{CN}}\right)$.

\section{General Procedure for Homocoupling of Fluorinated Aryl Boronic Acid Pinacol Esters (ArF $\mathbf{n}-B p i n)$}

In air, into a dried vial that was equipped with a stirring bar contained $1.5 \mathrm{~mL}$ of toluene, was added the corresponding $\operatorname{ArF}_{\mathrm{n}}-\mathrm{Bpin}(0.6 \mathrm{mmol}), \mathrm{Pd}(\mathrm{OAc})_{2}(3 \mathrm{mg}, 0.012 \mathrm{mmol}, 2 \mathrm{~mol} \%)$, and $\mathrm{Ag}_{2} \mathrm{O}$ (139 mg, $0.60 \mathrm{mmol}, 1$ equiv). After sealing the vial, the suspension was stirred for $5 \mathrm{~h}$ at $75{ }^{\circ} \mathrm{C}$. After cooling to room temperature, the solvent was evaporated in vacuo and the residue was purified by flash column chromatography on silica gel (hexane) to obtain the corresponding product. 


\section{Experimental Procedures and Characterization of Products}

\section{Synthesis of perfluorobiphenyl (2a)}

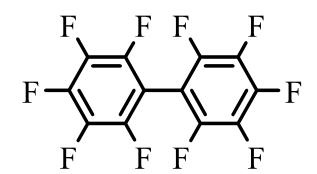

Compound 2a was synthesized following the general procedure and using the following chemicals and conditions: $\mathrm{C}_{6} \mathrm{~F}_{5} \mathrm{Bpin}(\mathbf{1 a})(176 \mathrm{mg}, 0.60 \mathrm{mmol}), \mathrm{Pd}(\mathrm{OAc})_{2}(3 \mathrm{mg}, 0.012$ mmol, $2 \mathrm{~mol} \%$ ), $\mathrm{Ag}_{2} \mathrm{O}$ (139 mg, $0.60 \mathrm{mmol}, 1$ equiv), and toluene (1.5 mL), $75^{\circ} \mathrm{C}, 5 \mathrm{~h}$. After flash column chromatography (hexane), product 2a was obtained as a white solid (97 mg, $97 \%)$.

${ }^{13} \mathbf{C}\left\{{ }^{19} \mathbf{F}\right\}$ NMR $\left(125 \mathrm{MHz}, \mathrm{CDCl}_{3}\right) \delta=144.8\left(\mathrm{C}_{\text {arom }}-\mathrm{F}_{\mathrm{o}}\right), 142.8\left(\mathrm{C}_{\text {arom }}-\mathrm{F}_{\mathrm{p}}\right), 138.1\left(\mathrm{C}_{\text {arom }}-\mathrm{F}_{\mathrm{m}}\right)$, $101.7\left(\mathrm{C}_{\text {arom }}-\mathrm{C}_{\text {arom }}\right) ;{ }^{19} \mathbf{F}\left\{{ }^{1} \mathbf{H}\right\}$ NMR $\left(376 \mathrm{MHz}, \mathrm{CDCl}_{3}\right) \delta=-160.3--160.2\left(m, 4 \mathrm{~F}_{\mathrm{m}}\right),-149.7$ $\left(t m,{ }^{3} J_{\mathrm{F}-\mathrm{F}}=21 \mathrm{~Hz}, 2 \mathrm{~F}_{\mathrm{p}}\right),-137.4--137.3\left(\mathrm{~m}, 4 \mathrm{~F}_{\mathrm{o}}\right)$; GC-MS: $[\mathrm{t}=6.492 \mathrm{~min}] \mathrm{m} / \mathrm{z}: 334[M]^{+}$; HRMS (APCI) calcd for $\mathrm{C}_{12} \mathrm{~F}_{10}[M]^{+}:$333.9835; found: 333.9825. Spectroscopic data matched those in the literature. ${ }^{\mathrm{S} 3}$

\section{Synthesis of $2,2^{\prime}, 4,4^{\prime}, 6,6^{\prime}-$ hexafluorobiphenyl (2b)}

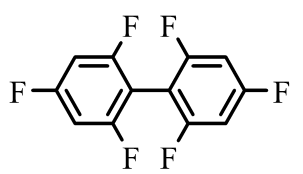

Compound $\mathbf{2 b}$ was synthesized following the general procedure and using the following chemicals and conditions: 2,4,6-trifluorophenylboronic acid pinacol ester (1b) (155 mg, 0.60 mmol), $\mathrm{Pd}(\mathrm{OAc})_{2}(3 \mathrm{mg}, 0.012 \mathrm{mmol}, 2 \mathrm{~mol} \%), \mathrm{Ag}_{2} \mathrm{O}$ (139 mg, $0.6 \mathrm{mmol}, 1$ equiv), and toluene $(1.5 \mathrm{~mL}), 75^{\circ} \mathrm{C}, 5 \mathrm{~h}$. After flash column chromatography (hexane), product $\mathbf{2 b}$ was obtained as a white solid ( $75 \mathrm{mg}, 96 \%)$.

${ }^{1} \mathbf{H}$ NMR $\left(300 \mathrm{MHz}, \mathrm{C}_{6} \mathrm{D}_{6}\right) \delta=6.32-6.22\left(m, 4 \mathrm{H}, \mathrm{C}_{\text {arom }}-\mathrm{H}_{\mathrm{m}}\right) ;{ }^{13} \mathbf{C}\left\{{ }^{1} \mathbf{H}\right\}$ NMR $(125 \mathrm{MHz}$, $\left.\mathrm{CDCl}_{3}\right) \delta=163.6\left(d t,{ }^{1} J_{\mathrm{F}-\mathrm{C}}=251 \mathrm{~Hz},{ }^{3} J_{\mathrm{F}-\mathrm{C}}=15 \mathrm{~Hz}, \mathrm{C}_{\mathrm{arom}}-\mathrm{F}_{\mathrm{p}}\right), 160.9\left(d m,{ }^{1} J_{\mathrm{F}-\mathrm{C}}=251 \mathrm{~Hz}\right.$ 
$\left.\mathrm{C}_{\text {arom }}-\mathrm{F}_{\mathrm{o}}\right), 102.4\left(m, \mathrm{C}_{\text {arom }}-\mathrm{C}_{\text {arom }}\right), 100.7\left(m, \mathrm{C}_{\text {arom }}-\mathrm{H}_{\mathrm{m}}\right) ;{ }^{3} \mathbf{C}\left\{{ }^{19} \mathbf{F}\right\}$ NMR $\left(125 \mathrm{MHz}, \mathrm{CDCl}_{3}\right) \delta=$ $163.6\left(t,{ }^{2} J_{\mathrm{H}-\mathrm{C}}=6 \mathrm{~Hz}, \mathrm{C}_{\text {arom }}-\mathrm{F}_{\mathrm{p}}\right), 160.9\left(t,{ }^{2} J_{\mathrm{H}-\mathrm{C}}=3 \mathrm{~Hz}, \mathrm{C}_{\text {arom }}-\mathrm{F}_{\mathrm{o}}\right), 102.4\left(t t,{ }^{3} J_{\mathrm{H}-\mathrm{C}}=5 \mathrm{~Hz},{ }^{4} J_{\mathrm{H}-\mathrm{C}}\right.$ $\left.=1 \mathrm{~Hz}, \mathrm{C}_{\text {arom }}-\mathrm{C}_{\text {arom }}\right), 100.7\left(d d,{ }^{1} J_{\mathrm{H}-\mathrm{C}}=169 \mathrm{~Hz},{ }^{3} J_{\mathrm{H}-\mathrm{C}}=4 \mathrm{~Hz}, \mathrm{C}_{\text {arom }}-\mathrm{H}_{\mathrm{m}}\right) ;{ }^{19} \mathbf{F}\left\{{ }^{1} \mathbf{H}\right\} \mathbf{N M R}(376$ $\left.\mathrm{MHz}, \mathrm{CDCl}_{3}\right) \delta=-107.4--107.3\left(m, 4 \mathrm{~F}_{\mathrm{o}}\right),-106.1--106.0\left(m, 2 \mathrm{~F}_{\mathrm{p}}\right)$; GC-MS: $[\mathrm{t}=4.520$ $\min ] \mathrm{m} / \mathrm{z}: 262[M]^{+}$; HRMS (APCI) calcd for $\mathrm{C}_{12} \mathrm{H}_{3} \mathrm{~F}_{6}[M-\mathrm{H}]^{-}:$261.0144; found: 261.0146. Spectroscopic data matched those in the literature. ${ }^{\mathrm{S} 3}$

\section{Synthesis of $2,2^{\prime}, 3,3^{\prime}, 4,4^{\prime}, 6,6^{\prime}-$-octafluorobiphenyl (2c)}

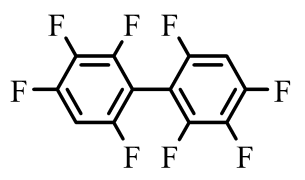

Compound 2c was synthesized following general procedure and using following chemicals and conditions: 2,3,4,6-tetrafluorophenylboronic acid pinacol ester (1c) (179 mg, 0.60 mmol), $\mathrm{Pd}(\mathrm{OAc})_{2}(3 \mathrm{mg}, 0.012 \mathrm{mmol}, 2 \mathrm{~mol} \%), \mathrm{Ag}_{2} \mathrm{O}$ (139 mg, $0.6 \mathrm{mmol}, 1$ equiv), and toluene $(1.5 \mathrm{~mL}), 75^{\circ} \mathrm{C}, 5 \mathrm{~h}$. After column chromatography (hexane) product 2c was obtained as colorless oil (83 mg, 93\%).

${ }^{1} \mathbf{H}$ NMR $\left(500 \mathrm{MHz}, \mathrm{CDCl}_{3}\right) \delta=6.96-6.91\left(m, 2 \mathrm{H}, \mathrm{C}_{\mathrm{arom}}-\mathrm{H}_{\mathrm{m}}\right) ;{ }^{13} \mathbf{C}\left\{{ }^{1} \mathbf{H}\right\} \mathbf{N M R}(125 \mathrm{MHz}$ $\left.\mathrm{CDCl}_{3}\right) \delta=101.4\left(\mathrm{~m}, \mathrm{C}_{\mathrm{arom}}-\mathrm{H}_{\mathrm{m}}\right)$, chemical shifts of $\mathrm{C}-\mathrm{F}$ bonds were not detected (but see ${ }^{13} \mathbf{C}\left\{{ }^{19} \mathbf{F}\right\}$ NMR spectra below $) ;{ }^{13} \mathbf{C}\left\{{ }^{19} \mathbf{F}\right\}$ NMR $\left(125 \mathrm{MHz}, \mathrm{CDCl}_{3}\right) \delta=154.9\left(d,{ }^{2} J_{\mathrm{H}-\mathrm{C}}=7 \mathrm{~Hz}\right.$, $\left.\mathrm{C}_{\text {arom }}-\mathrm{F}_{\mathrm{o}}{ }^{\prime}\right), 151.9\left(d,{ }^{2} J_{\mathrm{H}-\mathrm{C}}=7 \mathrm{~Hz}, \mathrm{C}_{\text {arom }}-\mathrm{F}_{\mathrm{p}}\right), 149.5\left(\mathrm{C}_{\text {arom }}-\mathrm{F}_{\mathrm{o}}\right), 137.6\left(d,{ }^{3} J_{\mathrm{H}-\mathrm{C}}=7 \mathrm{~Hz}, \mathrm{C}_{\text {arom }}{ }^{-}\right.$ $\left.\mathrm{F}_{\mathrm{m}}\right), 102.7\left(d,{ }^{3} J_{\mathrm{H}-\mathrm{C}}=6 \mathrm{~Hz}, \mathrm{C}_{\text {arom }}-\mathrm{C}_{\text {arom }}\right), 101.4\left(d,{ }^{1} J_{\mathrm{H}-\mathrm{C}}=170 \mathrm{~Hz}, \mathrm{C}_{\text {arom }}-\mathrm{H}_{\mathrm{m}}\right) ;{ }^{19} \mathbf{F}\left\{{ }^{1} \mathbf{H}\right\} \mathbf{N M R}$ (376 MHz, CDCl $\left.\mathbf{l}_{3}\right) \delta=-163.9--163.7\left(m, 2 \mathrm{~F}_{\mathrm{m}}\right),-130.4--130.3\left(m, 2 \mathrm{~F}_{\mathrm{o}}\right),-128.9\left(d m,{ }^{3} J_{\mathrm{F}-\mathrm{F}}\right.$ $\left.=23 \mathrm{~Hz}, 2 \mathrm{~F}_{\mathrm{p}}\right),-113.7--113.6\left(\mathrm{~m}, 2 \mathrm{~F}_{\mathrm{o}}{ }^{\prime}\right) ; \mathbf{G C}-\mathbf{M S}:[\mathrm{t}=6.860 \mathrm{~min}] \mathrm{m} / \mathrm{z}: 298[\mathrm{M}]^{+} ; \mathbf{H R M S}$ (APCI) calcd for $\mathrm{C}_{12} \mathrm{H}_{1} \mathrm{~F}_{8}[M-\mathrm{H}]^{-}:$:296.9956; found: 296.9956. Spectroscopic data matched those in the literature. ${ }^{\mathrm{S} 4}$ 


\section{Synthesis of $2,2^{\prime}, 3,3^{\prime}, 5,5^{\prime}, 6,6^{\prime}$-octafluorobiphenyl (2d)}

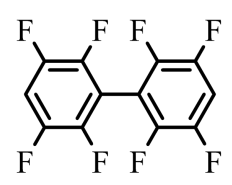

Compound 2d was synthesized following general procedure and using following chemicals and conditions: 2,3,5,6-tetrafluorophenylboronic acid pinacol ester (1d) (179 mg, $0.6 \mathrm{mmol}$ ), $\mathrm{Pd}(\mathrm{OAc})_{2}$ (3 mg, $0.012 \mathrm{mmol}, 2 \mathrm{~mol} \%$ ), $\mathrm{Ag}_{2} \mathrm{O}$ (139 mg, $0.6 \mathrm{mmol}, 1$ equiv), and toluene (1.5 $\mathrm{mL}), 75^{\circ} \mathrm{C}, 5 \mathrm{~h}$. After flash column chromatography (hexane) product $\mathbf{2 d}$ was obtained as white solid (86 mg, 97\%). White colorless single crystals suitable for X-ray diffraction were obtained by slow evaporation of a hexane solution at ambient temperature.

${ }^{1} \mathbf{H}$ NMR $\left(500 \mathrm{MHz}, \mathrm{C}_{6} \mathrm{D}_{6}\right) \delta=6.10-6.16\left(m, 2 \mathrm{H}, \mathrm{C}_{\mathrm{arom}}-\mathrm{H}_{\mathrm{p}}\right) ;{ }^{13} \mathbf{C}\left\{{ }^{1} \mathbf{H}\right\}$ NMR $(125 \mathrm{MHz}$, $\left.\mathrm{C}_{6} \mathrm{D}_{6}\right) \delta=146.1\left(d m,{ }^{1} J_{\mathrm{F}-\mathrm{C}}=240 \mathrm{MHz}, \mathrm{C}_{\mathrm{arom}}-\mathrm{F}_{\mathrm{o}}\right), 144.2\left(d m,{ }^{1} J_{\mathrm{F}-\mathrm{C}}=245 \mathrm{MHz}, \mathrm{C}_{\text {arom }}-\mathrm{F}_{\mathrm{p}}\right)$, $108.0\left(t,{ }^{1} J_{\mathrm{F}-\mathrm{C}}=23 \mathrm{~Hz}, \mathrm{C}_{\text {arom }}-\mathrm{H}_{\mathrm{p}}\right) ;{ }^{13} \mathbf{C}\left\{{ }^{\mathbf{1 9}} \mathbf{F}\right\} \mathbf{N M R}\left(125 \mathrm{MHz}, \mathrm{C}_{6} \mathrm{D}_{6}\right) \delta=146.1\left(d,{ }^{3} J_{\mathrm{H}-\mathrm{C}}=7\right.$ $\left.\mathrm{Hz}, \mathrm{C}_{\mathrm{arom}}-\mathrm{F}_{\mathrm{o}}\right), 144.2\left(d,{ }^{2} J_{\mathrm{H}-\mathrm{C}}=9 \mathrm{~Hz}, \mathrm{C}_{\text {arom }}-\mathrm{F}_{\mathrm{m}}\right), 108.0\left(d,{ }^{1} J_{\mathrm{H}-\mathrm{C}}=108 \mathrm{~Hz}, \mathrm{C}_{\text {arom }}-\mathrm{H}_{\mathrm{p}}\right), 107.9(d$, $\left.{ }^{4} J_{\mathrm{H}-\mathrm{C}}=1 \mathrm{~Hz}\right) ;{ }^{19} \mathbf{F}\left\{{ }^{1} \mathbf{H}\right\}$ NMR $\left(376 \mathrm{MHz}, \mathrm{C}_{6} \mathrm{D}_{6}\right) \delta=-139.2--139.1\left(m, 4 \mathrm{~F}_{\mathrm{o}}\right),-137.8--137.7$ ( $m$, 4F ); GC-MS: $[\mathrm{t}=7.157 \mathrm{~min}] \mathrm{m} / \mathrm{z}: 298[M]^{+}$; HRMS (APCI) calcd for $\mathrm{C}_{12} \mathrm{H}_{2} \mathrm{~F}_{8}[M]^{+}$: 298.0023; found: 298.0015. Spectroscopic data matched those in the literature. ${ }^{\mathrm{S} 5}$

\section{Synthesis of 2,2',6,6'-tetrafluorobiphenyl (2e)}

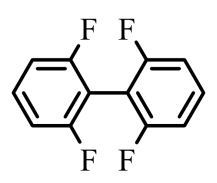

Compound 2e was synthesized following general procedure and using following chemicals and conditions: 2,6-difluorophenylboronic acid pinacol ester (1e) (136 mg, $0.60 \mathrm{mmol}$, $\mathrm{Pd}(\mathrm{OAc})_{2}(3 \mathrm{mg}, 0.012 \mathrm{mmol}, 2 \mathrm{~mol} \%), \mathrm{Ag}_{2} \mathrm{O}$ (139.04 mg, $\left.0.6 \mathrm{mmol}\right)$, and toluene $(1.5 \mathrm{~mL})$, $75^{\circ} \mathrm{C}, 5 \mathrm{~h}$. After flash column chromatography (hexane) product $2 \mathrm{e}$ was obtained as colorless oil (61 mg, 90\%). 
${ }^{1} \mathbf{H}$ NMR $\left(500 \mathrm{MHz}, \mathrm{CDCl}_{3}\right) \delta=7.43-7.38\left(m, 2 \mathrm{H}, \mathrm{C}_{\text {arom }}-\mathrm{H}_{\mathrm{p}}\right), 7.04-7.01\left(m, 4 \mathrm{H}, \mathrm{C}_{\text {arom- }}{ }^{-}\right.$ $\left.\mathrm{H}_{\mathrm{m}}\right) ;{ }^{13} \mathbf{C}\left\{{ }^{1} \mathbf{H}\right\}$ NMR $\left(125 \mathrm{MHz}, \mathrm{CDCl}_{3}\right) \delta=160.7\left(d m,{ }^{1} J_{\mathrm{F}-\mathrm{C}}=252 \mathrm{~Hz}, \mathrm{C}_{\text {arom }}-\mathrm{F}_{\mathrm{o}}\right), 131.0-$ $130.9\left(m, \mathrm{C}_{\mathrm{arom}}-\mathrm{H}_{\mathrm{p}}\right), 111.7-111.4\left(m, \mathrm{C}_{\mathrm{arom}}-\mathrm{H}_{\mathrm{m}}\right), 106.8\left(t,{ }^{2} J_{\mathrm{F}-\mathrm{C}}=21 \mathrm{~Hz}, \mathrm{C}_{\text {arom }}-\mathrm{C}_{\text {arom }}\right)$; ${ }^{13} \mathbf{C}\left\{{ }^{19} \mathbf{F}\right\}$ NMR $\left(125 \mathrm{MHz}, \mathrm{CDCl}_{3}\right) \delta=160.7\left(d m,{ }^{2} J_{\mathrm{H}-\mathrm{C}}=13 \mathrm{~Hz}, \mathrm{C}_{\text {arom }}-\mathrm{F}_{\mathrm{o}}\right), 131.0\left(d,{ }^{1} J_{\mathrm{H}-\mathrm{C}}=\right.$ $\left.164 \mathrm{~Hz}, \mathrm{C}_{\mathrm{arom}}-\mathrm{H}_{\mathrm{p}}\right), 111.5\left(d d d,{ }^{1} J_{\mathrm{H}-\mathrm{C}}=166 \mathrm{~Hz},{ }^{2} J_{\mathrm{H}-\mathrm{C}}=8 \mathrm{~Hz},{ }^{3} J_{\mathrm{H}-\mathrm{C}}=1 \mathrm{~Hz}, \mathrm{C}_{\mathrm{arom}}-\mathrm{H}_{\mathrm{m}}\right), 106.8-$ $106.7\left(m, \mathrm{C}_{\text {arom }}-\mathrm{C}_{\text {arom }}\right) ;{ }^{19} \mathbf{F}\left\{{ }^{1} \mathbf{H}\right\} \mathbf{N M R}\left(376 \mathrm{MHz}, \mathrm{CDCl}_{3}\right) \delta=-110.6\left(s, 4 \mathrm{~F}_{\mathrm{o}}\right) ; \mathbf{G C}-\mathbf{M S}:[\mathrm{t}=$ $7.797 \mathrm{~min}] \mathrm{m} / \mathrm{z}: 226[M]^{+}$; HRMS (APCI) calcd for $\mathrm{C}_{12} \mathrm{H}_{6} \mathrm{~F}_{4}\left[M^{+}\right]$: 226.0400; found: 226.0397. Spectroscopic data matched those in the literature. ${ }^{\mathrm{S} 6}$

\section{$2,2^{\prime}, 3,3^{\prime}, 4,4^{\prime}$-hexafluorobiphenyl (2f)}

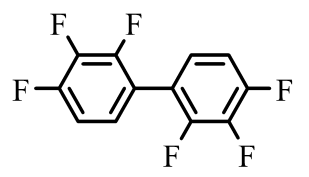

Compound $2 \mathbf{f}$ was synthesized following general procedure and using following chemicals and conditions: 2,3,4-trifluorophenylboronic acid pinacol ester (157 mg, $0.6 \mathrm{mmol}), \mathrm{Pd}(\mathrm{OAc})_{2}(3 \mathrm{mg}, 0.012 \mathrm{mmol}, 2 \mathrm{~mol} \%), \mathrm{Ag}_{2} \mathrm{O}$ (139 mg, $0.6 \mathrm{mmol}, 1$ equiv), and toluene (1.5 mL), $75{ }^{\circ} \mathrm{C}, 5 \mathrm{~h}$. After flash column chromatography (hexane), product 2 f was obtained as white solid (76 mg, 97\%). White colorless single crystals suitable for X-ray diffraction were obtained by slow evaporation of a hexane solution at ambient temperature.

${ }^{1} \mathbf{H}$ NMR $\left(300 \mathrm{MHz}, \mathrm{C}_{6} \mathrm{D}_{6}\right) \delta=6.36-6.23\left(m, 4 \mathrm{H}, \mathrm{C}_{\mathrm{arom}}-\mathrm{H}_{\mathrm{o}, \mathrm{m}}\right) ;{ }^{13} \mathbf{C}\left\{{ }^{1} \mathbf{H}\right\}$ NMR $(125 \mathrm{MHz}$ $\left.\mathrm{C}_{6} \mathrm{D}_{6}\right) \delta=151.5\left(d d d,{ }^{1} J_{\mathrm{F}-\mathrm{C}}=251 \mathrm{~Hz},{ }^{2} J_{\mathrm{F}-\mathrm{C}}=10 \mathrm{~Hz},{ }^{3} J_{\mathrm{F}-\mathrm{C}}=3 \mathrm{~Hz}, \mathrm{C}_{\mathrm{arom}}-\mathrm{F}_{\mathrm{p}}\right), 149.2\left(d m,{ }^{1} J_{\mathrm{F}-\mathrm{C}}=\right.$ $\left.254 \mathrm{~Hz}, \mathrm{C}_{\mathrm{arom}}-\mathrm{F}_{\mathrm{o}}\right), 140.6\left(d t,{ }^{1} J_{\mathrm{F}-\mathrm{C}}=252 \mathrm{~Hz},{ }^{2} J_{\mathrm{F}-\mathrm{C}}=16 \mathrm{~Hz}, \mathrm{C}_{\mathrm{arom}}-\mathrm{F}_{\mathrm{m}}\right), 124.9\left(\right.$ oct,${ }^{2} J_{\mathrm{F}-\mathrm{C}}=2$ $\left.\mathrm{Hz}, \mathrm{C}_{\mathrm{arom}}-\mathrm{H}_{\mathrm{m}}\right), 119.4\left(m, \mathrm{C}_{\text {arom }}-\mathrm{C}_{\mathrm{arom}}\right), 112.3\left(d d,{ }^{3} J_{\mathrm{F}-\mathrm{C}}=18 \mathrm{~Hz},{ }^{4} J_{\mathrm{F}-\mathrm{C}}=4 \mathrm{~Hz}, \mathrm{C}_{\text {arom }}-\mathrm{H}_{\mathrm{o}}\right)$; ${ }^{13} \mathbf{C}\left\{{ }^{19} \mathbf{F}\right\}$ NMR $\left(125 \mathrm{MHz}, \mathrm{C}_{6} \mathrm{D}_{6}\right) \delta=151.5\left(d d,{ }^{2} J_{\mathrm{H}-\mathrm{C}}=12 \mathrm{~Hz},{ }^{3} J_{\mathrm{H}-\mathrm{C}}=4 \mathrm{~Hz}, \mathrm{C}_{\mathrm{arom}}-\mathrm{F}_{\mathrm{p}}\right), 149.2$ $\left(d,{ }^{3} J_{\mathrm{H}-\mathrm{C}}=11 \mathrm{~Hz}, \mathrm{C}_{\text {arom }}-\mathrm{F}_{\mathrm{o}}\right), 140.6\left(d,{ }^{3} J_{\mathrm{H}-\mathrm{C}}=8 \mathrm{~Hz}, \mathrm{C}_{\mathrm{arom}}-\mathrm{F}_{\mathrm{m}}\right), 124.9\left(d,{ }^{1} J_{\mathrm{H}-\mathrm{C}}=167 \mathrm{~Hz}, \mathrm{C}_{\text {arom }}{ }^{-}\right.$ 
$\left.\mathrm{H}_{\mathrm{m}}\right), 119.4\left(d d,{ }^{2} J_{\mathrm{H}-\mathrm{C}}=9 \mathrm{~Hz},{ }^{3} J_{\mathrm{H}-\mathrm{C}}=4 \mathrm{~Hz}, \mathrm{C}_{\text {arom }}-\mathrm{C}_{\text {arom }}\right), 112.3\left(d,{ }^{1} J_{\mathrm{H}-\mathrm{C}}=168 \mathrm{~Hz}, \mathrm{C}_{\text {arom }}-\mathrm{H}_{\mathrm{o}}\right)$; ${ }^{19} \mathbf{F}\left\{{ }^{1} \mathbf{H}\right\}$ NMR $\left(376 \mathrm{MHz}, \mathrm{C}_{6} \mathrm{D}_{6}\right) \delta=-159.4--159.3\left(m, 2 \mathrm{~F}_{\mathrm{m}}\right),-135.1--135.0\left(m, 2 \mathrm{~F}_{\mathrm{o}}\right),-$ $133.4\left(d m,{ }^{3} J_{\mathrm{F}-\mathrm{F}}=21 \mathrm{~Hz}, 2 \mathrm{~F}_{\mathrm{p}}\right)$; GC-MS: $[\mathrm{t}=8.119 \mathrm{~min}] \mathrm{m} / \mathrm{z}: 262[M]^{+}$; HRMS (APCI) calcd for $\mathrm{C}_{12} \mathrm{H}_{4} \mathrm{~F}_{6}[M]^{+}: 262.0212$; found: 262.0207 . Spectroscopic data matched those in the literature. ${ }^{\mathrm{S} 7}$

\section{Synthesis of $2,2^{\prime}, 5,5^{\prime}$-tetrafluorobiphenyl (2g)}

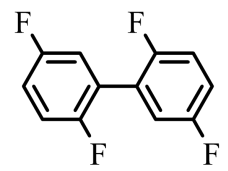

Compound $\mathbf{2 g}$ was synthesized following general procedure and using following chemicals and conditions: 2,5-difluorophenylboronic acid pinacol ester (1g) (136 mg, $0.60 \mathrm{mmol}$ ), $\mathrm{Pd}(\mathrm{OAc})_{2}(3 \mathrm{mg}, 0.012 \mathrm{mmol}, 2 \mathrm{~mol} \%), \mathrm{Ag}_{2} \mathrm{O}$ (139.04 mg, $\left.0.6 \mathrm{mmol}\right)$, and toluene $(1.5 \mathrm{~mL})$, $75^{\circ} \mathrm{C}, 5 \mathrm{~h}$. After flash column chromatography (hexane) product $\mathbf{2 g}$ was obtained as white solid (66 mg, 98\%).

${ }^{1} \mathbf{H}$ NMR $\left(300 \mathrm{MHz}, \mathrm{CDCl}_{3}\right) \delta=7.18-7.04\left(m, 6 \mathrm{H}, \mathrm{C}_{\mathrm{arom}}-\mathrm{H}_{\mathrm{o}, \mathrm{m}, \mathrm{p}}\right) ;{ }^{13} \mathbf{C}\left\{{ }^{1} \mathbf{H}\right\} \mathbf{N M R}(125 \mathrm{MHz}$ $\left.\mathrm{CDCl}_{3}\right) \delta=158.6\left(d d,{ }^{1} J_{\mathrm{F}-\mathrm{C}}=243 \mathrm{~Hz},{ }^{4} J_{\mathrm{F}-\mathrm{C}}=2 \mathrm{~Hz}, \mathrm{C}_{\mathrm{arom}}-\mathrm{F}_{\mathrm{m}}\right), 155.9\left(d d d,{ }^{1} J_{\mathrm{F}-\mathrm{C}}=247 \mathrm{~Hz},{ }^{4} J_{\mathrm{F}-}\right.$ $\left.\mathrm{C}=2 \mathrm{~Hz},{ }^{4} J_{\mathrm{F}-\mathrm{C}}=2 \mathrm{~Hz}, \mathrm{C}_{\text {arom }}-\mathrm{F}_{\mathrm{o}}\right), 123.9-123.7\left(m, \mathrm{C}_{\mathrm{arom}}-\mathrm{C}_{\mathrm{arom}}\right), 117.9\left(d t,{ }^{2} J_{\mathrm{F}-\mathrm{C}}=25 \mathrm{~Hz},{ }^{3} J_{\mathrm{F}-}\right.$ $\left.\mathrm{C}=3 \mathrm{~Hz}, \mathrm{C}_{\mathrm{arom}}-\mathrm{H}_{\mathrm{o}}\right), 117.4-117.0\left(m, \mathrm{C}_{\mathrm{arom}}-\mathrm{H}_{\mathrm{p}}\right), 116.8\left(d m,{ }^{2} J_{\mathrm{F}-\mathrm{C}}=24 \mathrm{~Hz}, \mathrm{C}_{\mathrm{arom}}-\mathrm{H}_{\mathrm{m}}\right)$ ${ }^{19} \mathbf{F}\left\{{ }^{1} \mathbf{H}\right\}$ NMR $\left(376 \mathrm{MHz}, \mathrm{CDCl}_{3}\right) \delta=-120.9--120.8\left(m, 2 \mathrm{~F}_{\mathrm{m}}\right),-118.9--118.8\left(m, 2 \mathrm{~F}_{\mathrm{o}}\right) ;$ GC-MS: $[\mathrm{t}=5.530 \mathrm{~min}] \mathrm{m} / \mathrm{z}: 226[M]^{+}$; HRMS (APCI) calcd. for $\mathrm{C}_{12} \mathrm{H}_{6} \mathrm{~F}_{4}\left[M^{+}\right]: 226.0400$; found: 226.0399 . Spectroscopic data matched those in the literature. ${ }^{\mathrm{S} 8}$

\section{3,3',5,5'-tetrakis(trifluoromethyl)biphenyl (2h)}




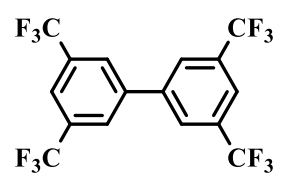

Compound $\mathbf{2 h}$ was synthesized following general procedure and using following chemicals and conditions: 3,5-di(trifluoromethyl)phenylboronic acid pinacol ester (1h) (204 mg, 0.60 $\mathrm{mmol}), \mathrm{Pd}(\mathrm{OAc})_{2}(3 \mathrm{mg}, 0.012 \mathrm{mmol}, 2 \mathrm{~mol} \%), \mathrm{Ag}_{2} \mathrm{O}$ (139.04 mg, $\left.0.6 \mathrm{mmol}\right)$, and toluene $(1.5 \mathrm{~mL}), 75^{\circ} \mathrm{C}, 5 \mathrm{~h}$. After flash column chromatography (hexane) product $\mathbf{2 h}$ was obtained as white solid (118 mg, 92\%).

${ }^{1} \mathbf{H}$ NMR $\left(500 \mathrm{MHz}, \mathrm{CDCl}_{3}\right) \delta=8.03\left(s, 4 \mathrm{H}, \mathrm{C}_{\text {arom }}-\mathrm{H}_{\mathrm{o}}\right), 7.99\left(s, 2 \mathrm{H}, \mathrm{C}_{\text {arom }}-\mathrm{H}_{\mathrm{p}}\right) ;{ }^{13} \mathbf{C}\left\{{ }^{1} \mathbf{H}\right\}$ NMR $\left(125 \mathrm{MHz}, \mathrm{CDCl}_{3}\right) \delta=140.6\left(\mathrm{C}_{\mathrm{arom}}-\mathrm{C}_{\mathrm{arom}}\right), 133.07\left(q,{ }^{2} J_{\mathrm{F}-\mathrm{C}}=34 \mathrm{~Hz}, C_{\mathrm{arom}}-\mathrm{CF}_{3}\right)$ $127.7\left(m, \mathrm{C}_{\mathrm{arom}}-\mathrm{H}_{\mathrm{o}}\right), 123.2\left(q,{ }^{1} J_{\mathrm{F}-\mathrm{C}}=273 \mathrm{~Hz}, \mathrm{CF}_{3}\right), 122.8\left(\right.$ quin, $\left.{ }^{3} J_{\mathrm{F}-\mathrm{C}}=4 \mathrm{~Hz}, \mathrm{C}_{\text {arom }}-\mathrm{H}_{\mathrm{p}}\right) ;{ }^{19} \mathbf{F}$ NMR $\left(470 \mathrm{MHz}, \mathrm{CDCl}_{3}\right) \delta=-62.9\left(s, 12 \mathrm{~F}, \mathrm{CF}_{3}\right)$; GC-MS: $[\mathrm{t}=6.985 \mathrm{~min}] \mathrm{m} / \mathrm{z}: 426[M]^{+}$; HRMS (APCI) calcd for $\mathrm{C}_{16} \mathrm{H}_{6} \mathrm{~F}_{12}[M]^{-}:$426.0283; found: 426.0275. Spectroscopic data matched those in the literature. ${ }^{\mathrm{S} 9}$

\section{A Test for the Possibility of C-F Oxidative Addition \\ Scheme S1.}

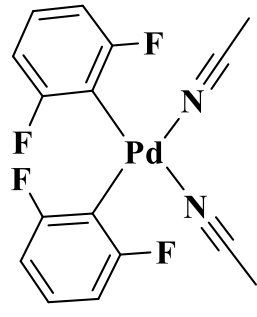

$3 \mathbf{e}$

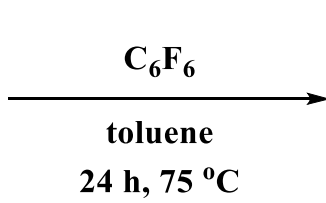

$24 \mathrm{~h}, 75^{\circ} \mathrm{C}$

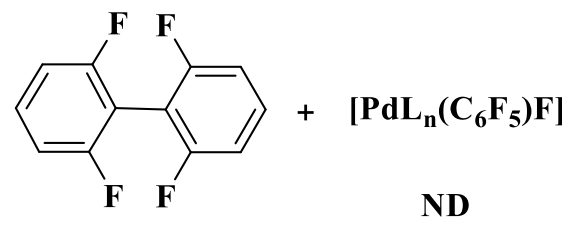

$2 \mathrm{e}$

Inside the glove box, under argon, into a J. Young NMR tube, were added compound 3e (8 $\mathrm{mg}, 0.02 \mathrm{mmol}), \mathrm{C}_{6} \mathrm{~F}_{6}(11 \mathrm{mg}, 0.06 \mathrm{mmol})$ and toluene $(0.4 \mathrm{~mL})$. After sealing, the NMR tube was taken out of the glove box, heated at $75{ }^{\circ} \mathrm{C}$ for $24 \mathrm{~h}$, and monitored by ${ }^{19} \mathrm{~F}$ NMR spectroscopy. The conversion to give 2e is complete after 10 minutes. However, after $24 \mathrm{~h}$, we did not detect any $\mathrm{Pd}$ complex arising from $\mathrm{C}-\mathrm{F}$ activation of $\mathrm{C}_{6} \mathrm{~F}_{6}$. The ${ }^{19} \mathrm{~F}$ NMR 
spectrum of our reaction mixture is shown below. According to the literature, ${ }^{\mathrm{S} 10}$ the ${ }^{19} \mathrm{~F}$ NMR chemical shifts for a typical $\left[\mathrm{PdL}_{2}\left(\mathrm{C}_{6} \mathrm{~F}_{5}\right) \mathrm{F}\right]$ complex such as trans $-\left[\mathrm{Pd}\left(\mathrm{Cy}_{3} \mathrm{P}\right)_{2}\left(\mathrm{C}_{6} \mathrm{~F}_{5}\right) \mathrm{F}\right]$ is $\delta=$ $-110.1\left(m, 2 \mathrm{~F}, \mathrm{C}_{6} \mathrm{~F}_{5}\right),-164.8\left(m, 1 \mathrm{~F}, \mathrm{C}_{6} \mathrm{~F}_{5}\right),-165.8\left(m, 2 \mathrm{~F}, \mathrm{C}_{6} \mathrm{~F}_{5}\right),-325.0$ (br s, 1F, Pd-F).

${ }^{19}$ F NMR spectrum of the reaction mixture in Scheme S1 $(-40--240 \mathrm{ppm}$ region)

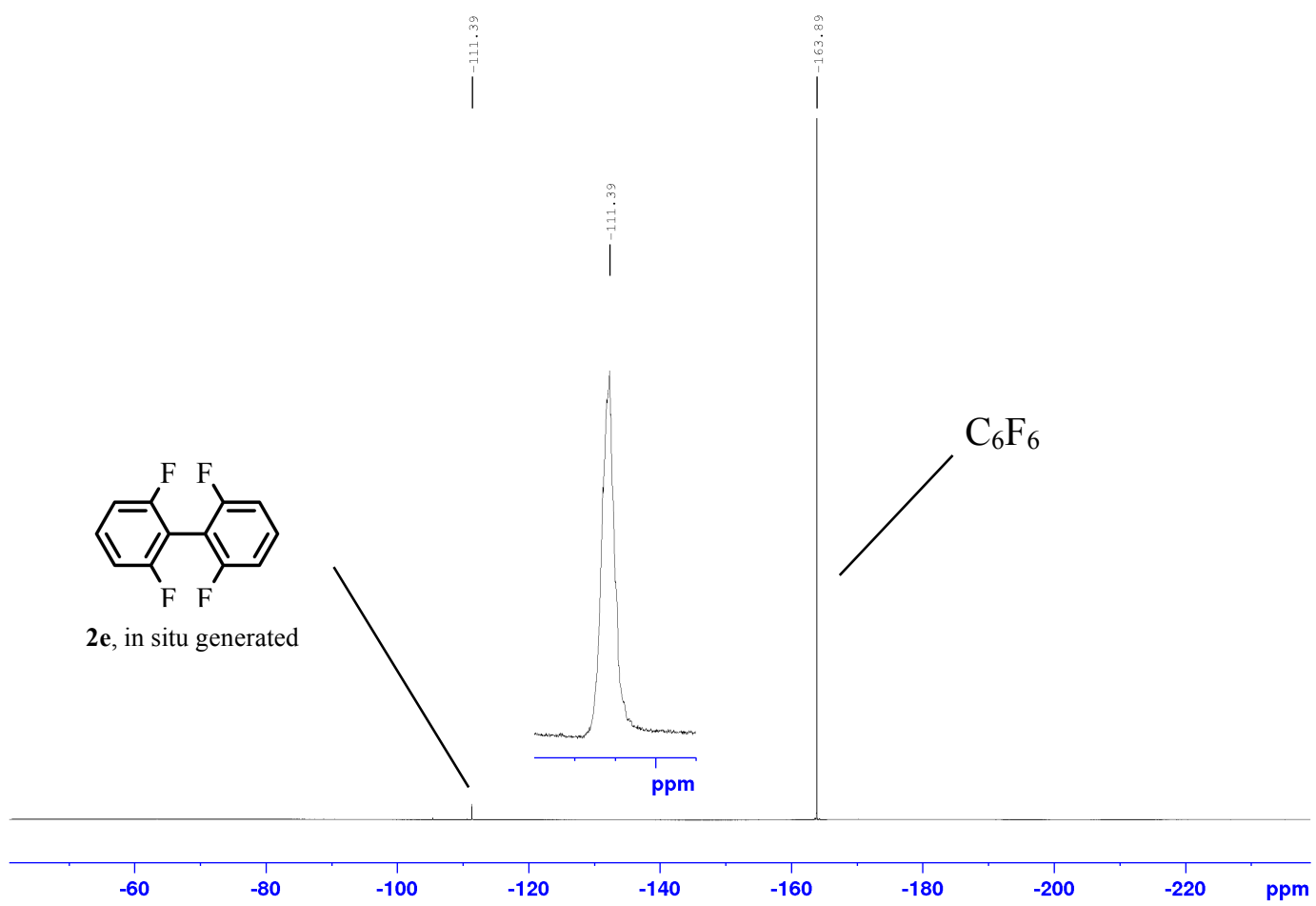

${ }^{19} \mathrm{~F}$ NMR spectrum of the reaction mixture in Scheme $S 1(-230--430 \mathrm{ppm}$ region) 


\begin{tabular}{|c|c|c|c|c|c|c|c|c|c|}
\hline $\begin{array}{c}1 \\
-240\end{array}$ & -260 & $\begin{array}{c}1 \\
-280\end{array}$ & -300 & -320 & $\begin{array}{c}1 \\
-340\end{array}$ & $\begin{array}{c}1 \\
-360\end{array}$ & -380 & -400 & ppm \\
\hline
\end{tabular}


9. ${ }^{1} \mathrm{H},{ }^{13} \mathrm{C}\left\{{ }^{1} \mathrm{H}\right\},{ }^{11} \mathrm{~B}\left\{{ }^{1} \mathrm{H}\right\},{ }^{19} \mathrm{~F},{ }^{19} \mathrm{~F}\left\{{ }^{1} \mathrm{H}\right\}$ NMR, IR Spectra and GC-MS Data ${ }^{1} \mathrm{H}$ NMR spectrum of $1 \mathrm{a}\left(300 \mathrm{MHz}, \mathrm{CDCl}_{3}\right)$<smiles></smiles>

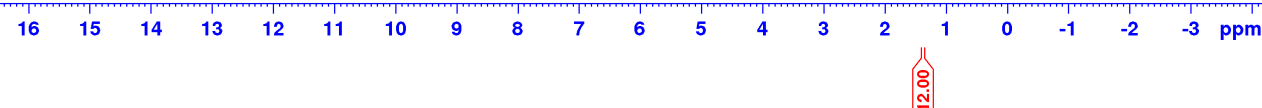

${ }^{13} \mathrm{C}\left\{{ }^{1} \mathrm{H}\right\}$ NMR spectrum of $1 \mathrm{a}\left(125 \mathrm{MHz}, \mathrm{CDCl}_{3}\right)$

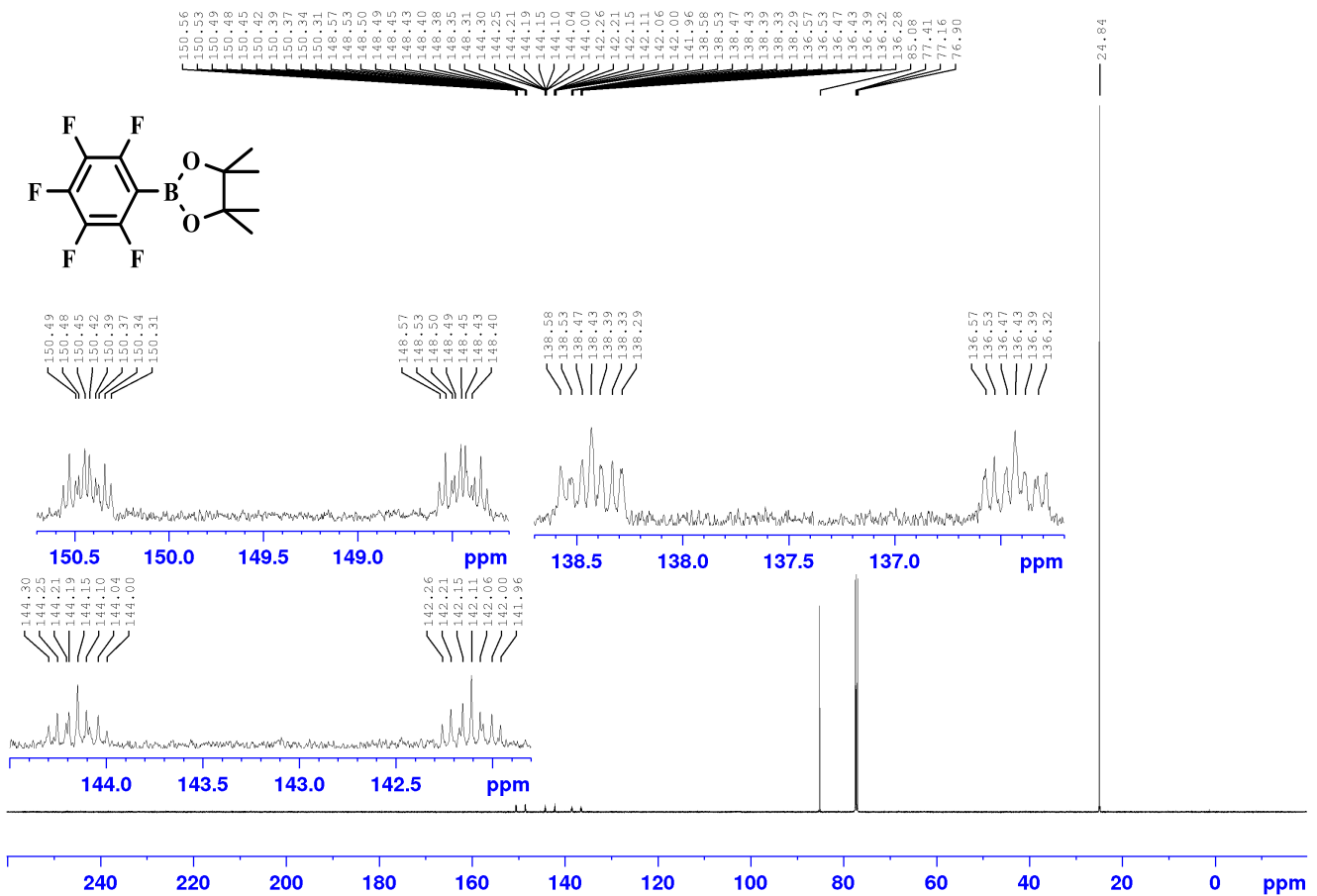


${ }^{19}$ F NMR spectrum of $1 \mathrm{a}\left(470 \mathrm{MHz}, \mathrm{CDCl}_{3}\right)$
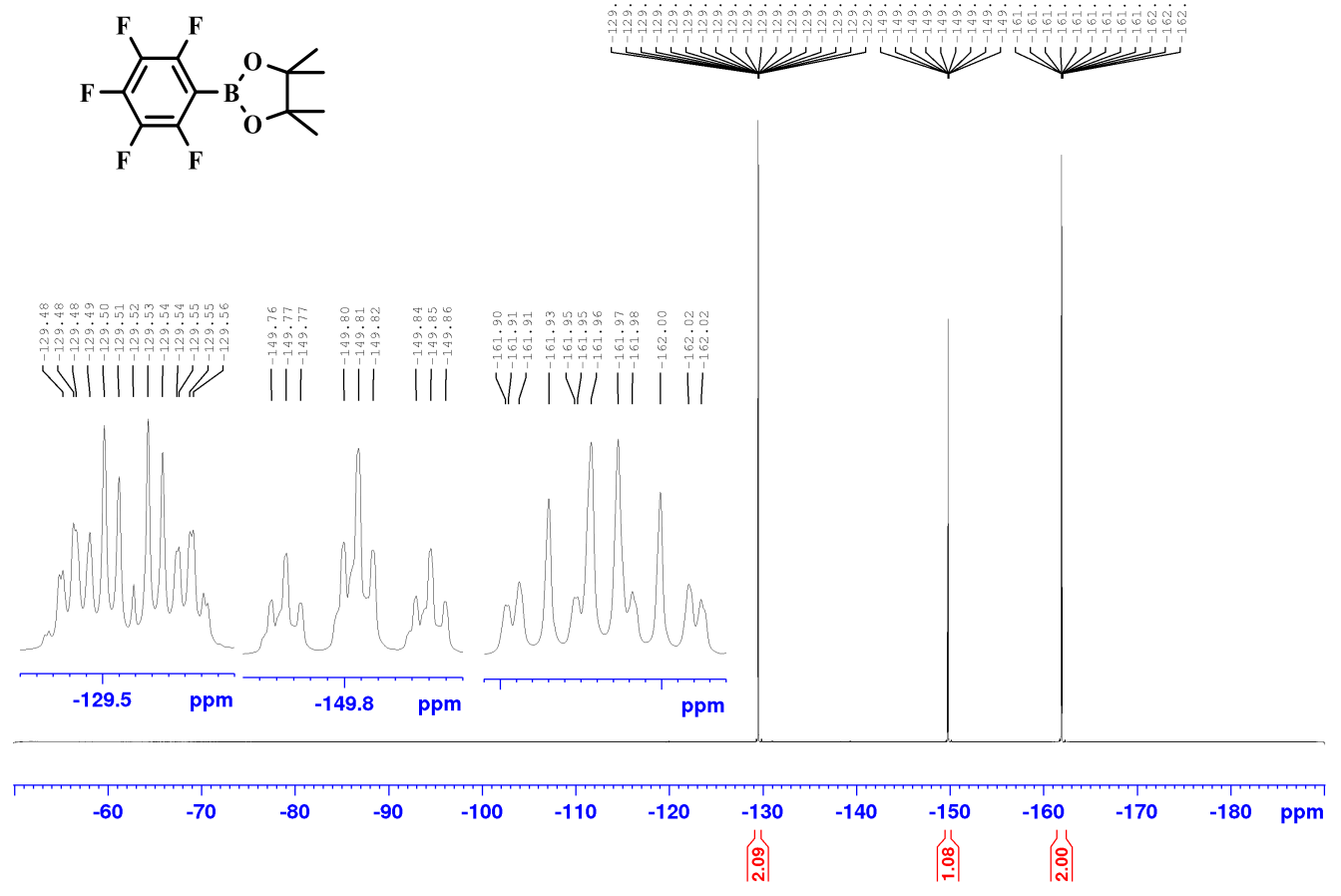

${ }^{11} \mathrm{~B}\left\{{ }^{1} \mathrm{H}\right\}$ NMR spectrum of $1 \mathrm{a}\left(96 \mathrm{MHz}, \mathrm{CDCl}_{3}\right)$
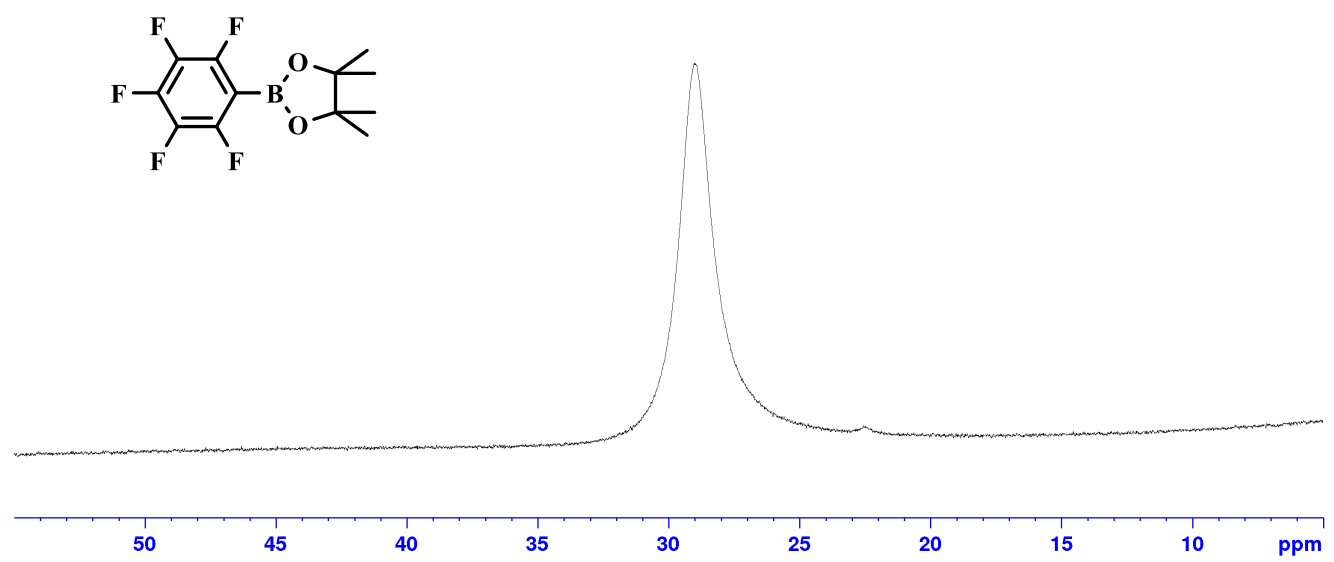


\section{GC-MS of 1a}

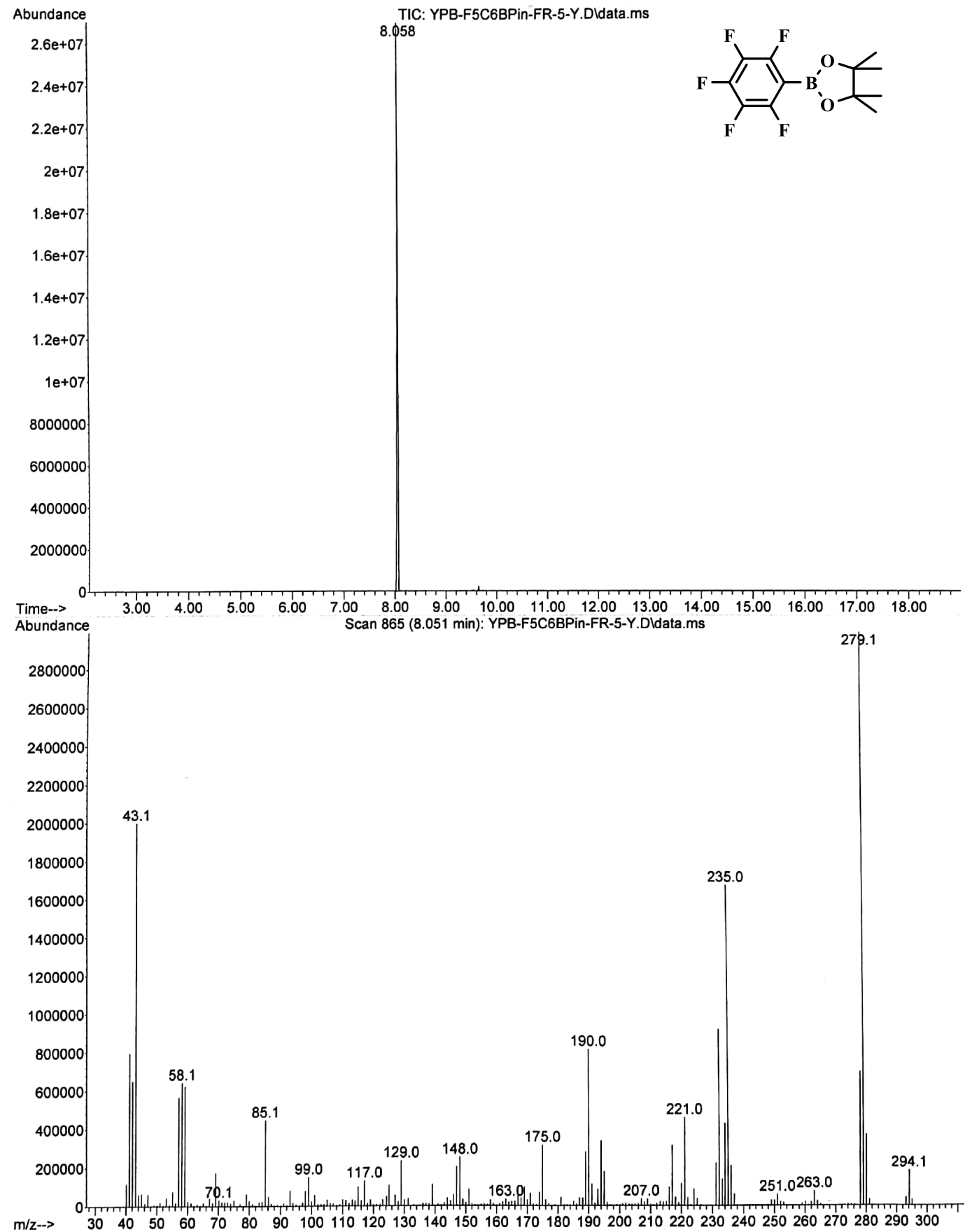


${ }^{1} \mathrm{H}$ NMR spectrum of 3a (500 MHz, DMSO- $\left.d_{6}\right)$
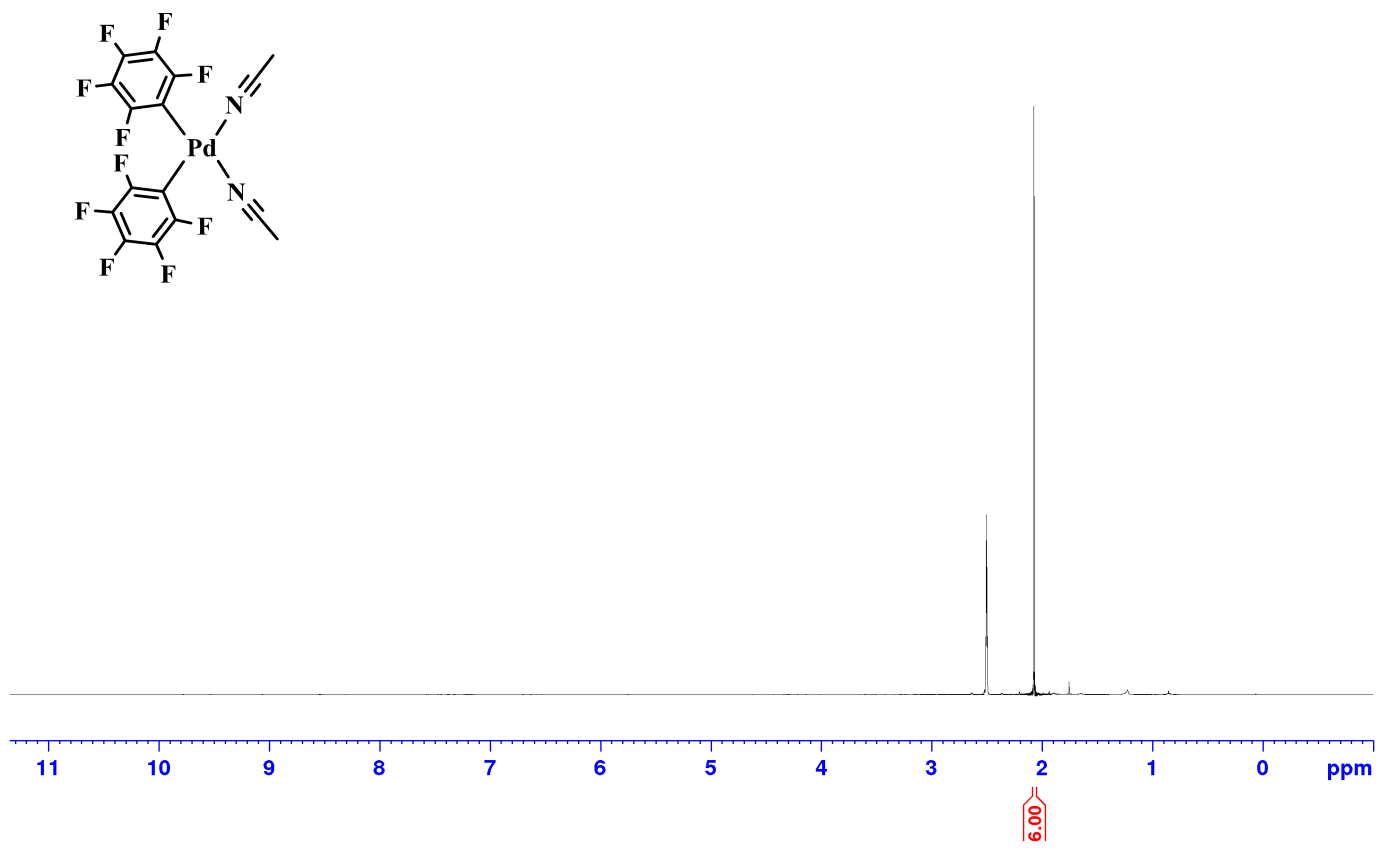

${ }^{13} \mathrm{C}\left\{{ }^{1} \mathrm{H}\right\}$ NMR spectrum of 3a (125 MHz, DMSO- $\left.d_{6}\right)$
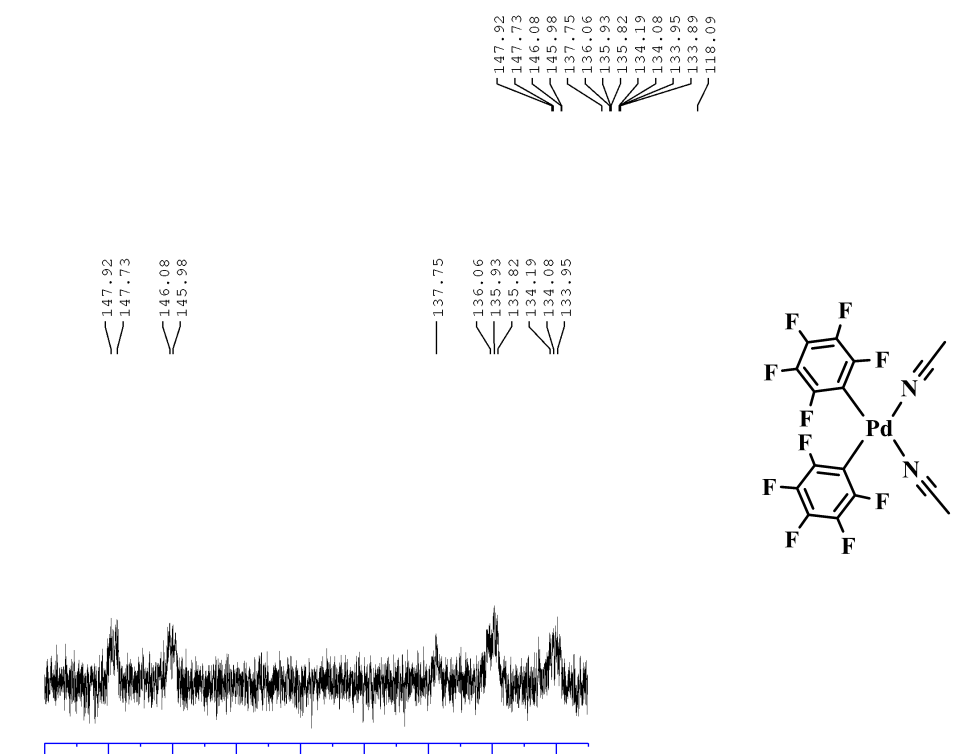

$\begin{array}{lllllllll}148 & 146 & 144 & 142 & 140 & 138 & 136 & \text { ppm }\end{array}$ 
${ }^{13} \mathrm{C}\left\{{ }^{19} \mathrm{~F}\right\}$ NMR spectrum of 3a (125 MHz, DMSO- $\left.d_{6}\right)$

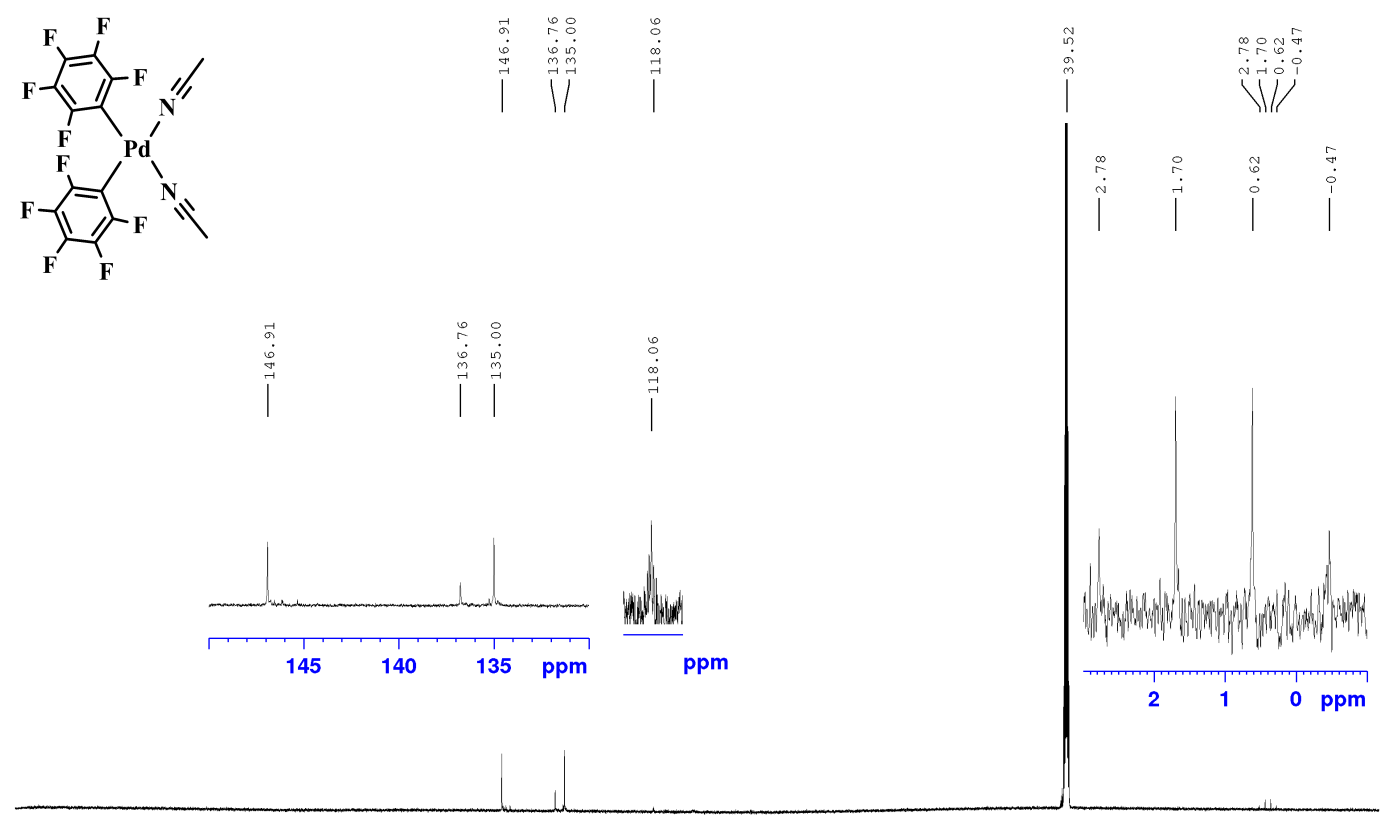

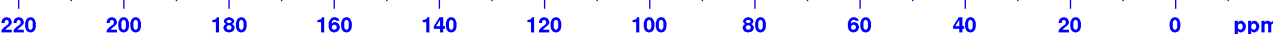

${ }^{13} \mathrm{C}\left\{{ }^{19} \mathrm{~F}\right\}$ NMR spectrum of $3 \mathrm{a}\left(125 \mathrm{MHz}, \mathrm{CD}_{3} \mathrm{CN}\right)$
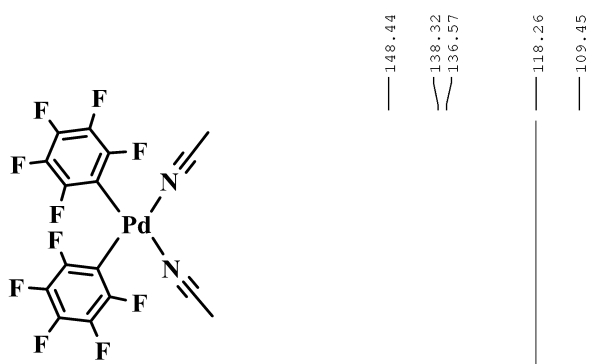
${ }^{19} \mathrm{~F}\left\{{ }^{1} \mathrm{H}\right\}$ NMR spectrum of $3 \mathrm{a}\left(376 \mathrm{MHz}, \mathrm{CD}_{3} \mathrm{CN}\right)$
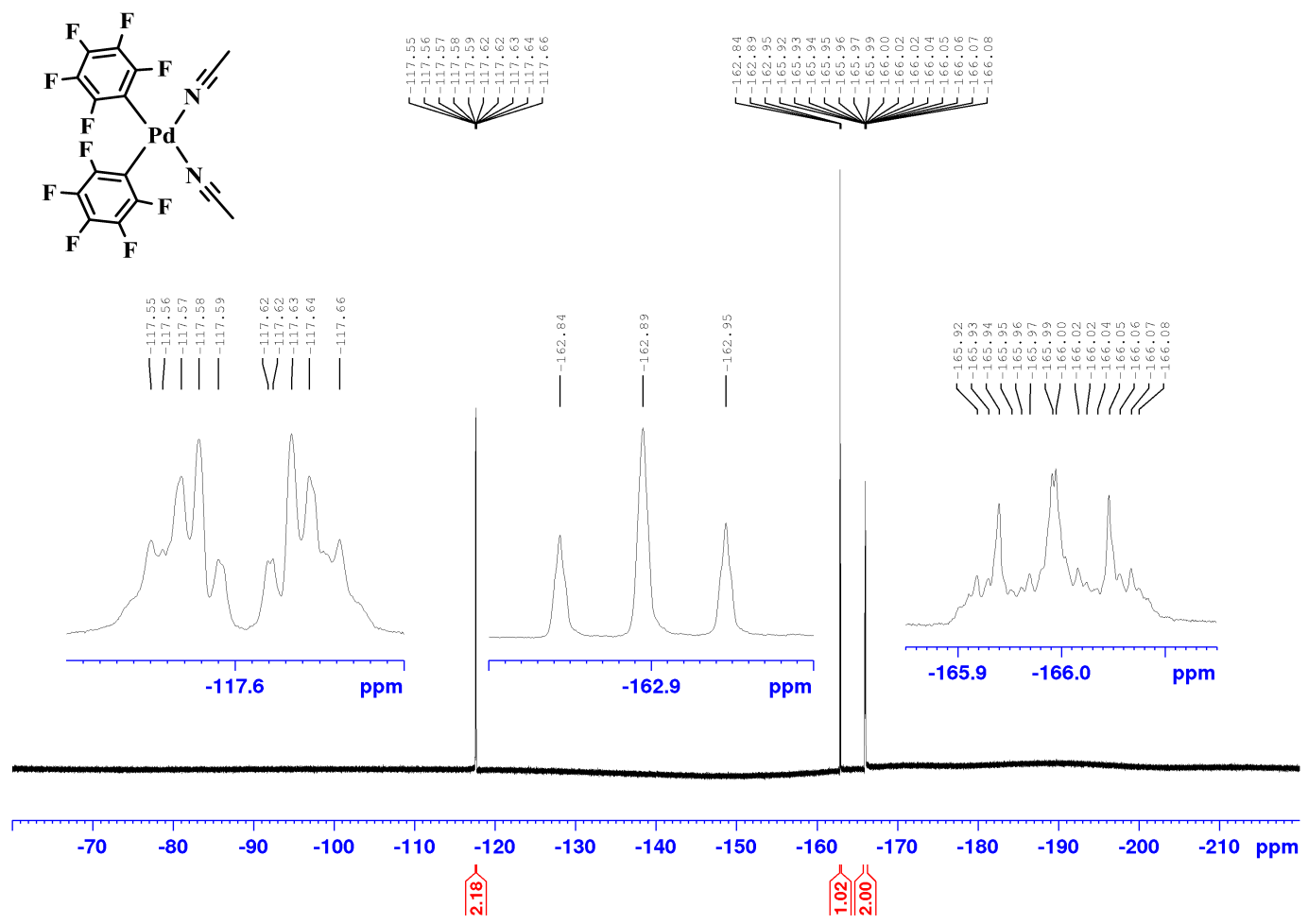

IR spectrum of 3a in the solid-state

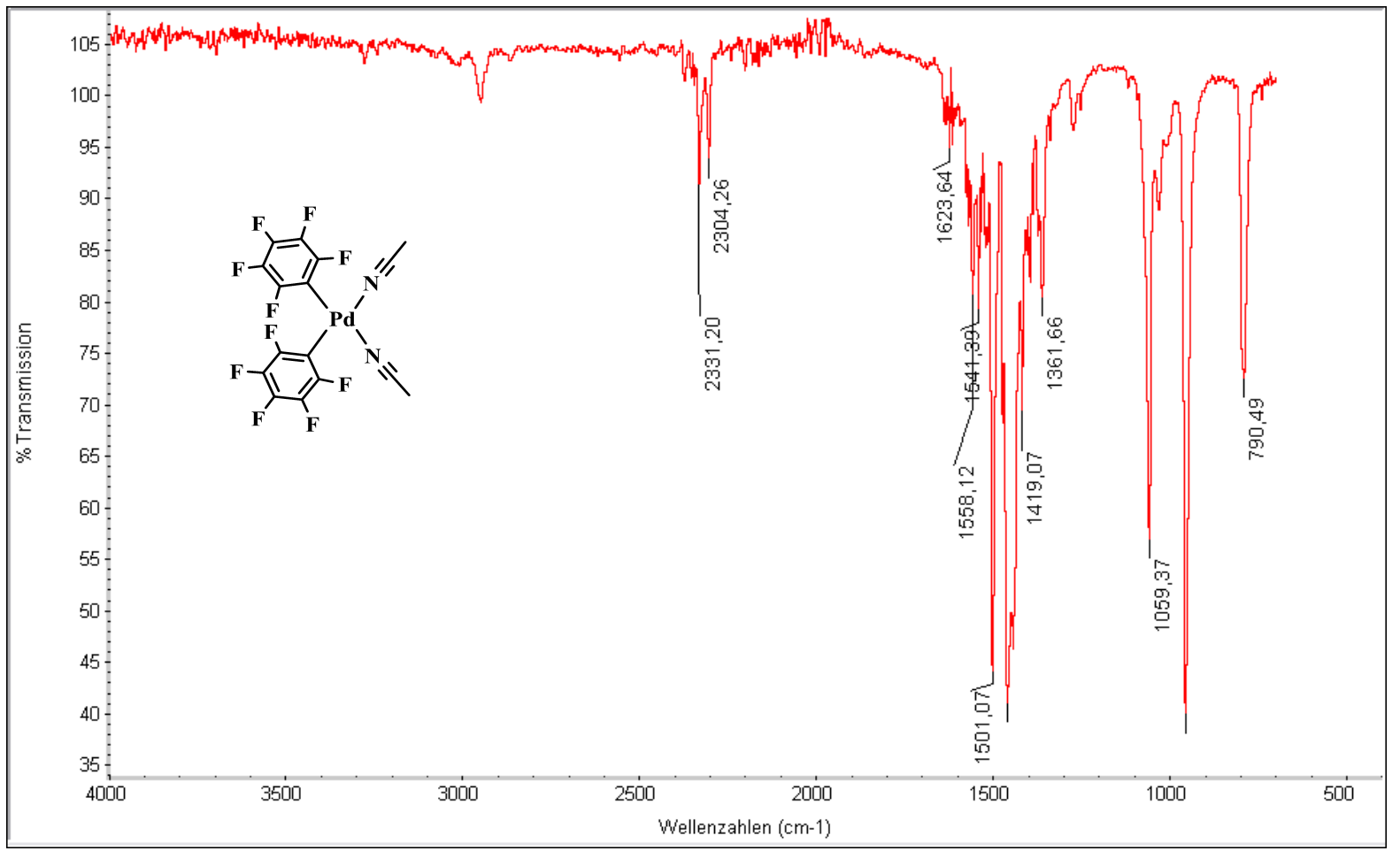


${ }^{1} \mathrm{H}$ NMR spectrum of $3 \mathrm{~b}\left(300 \mathrm{MHz}, \mathrm{CDCl}_{3}\right)$

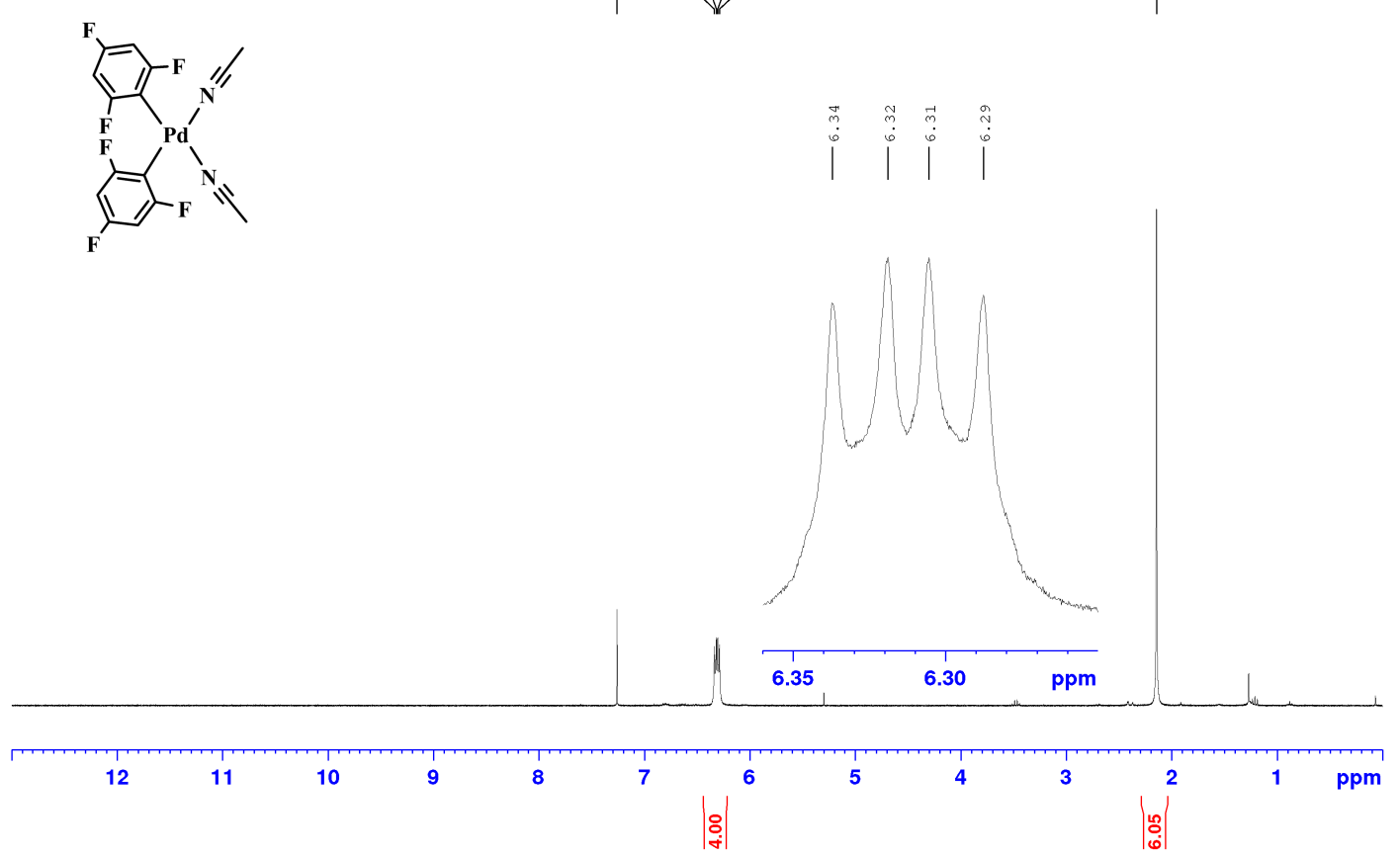

${ }^{13} \mathrm{C}\left\{{ }^{1} \mathrm{H}\right\}$ NMR spectrum of 3b (125 MHz, DMSO-d 6$)$
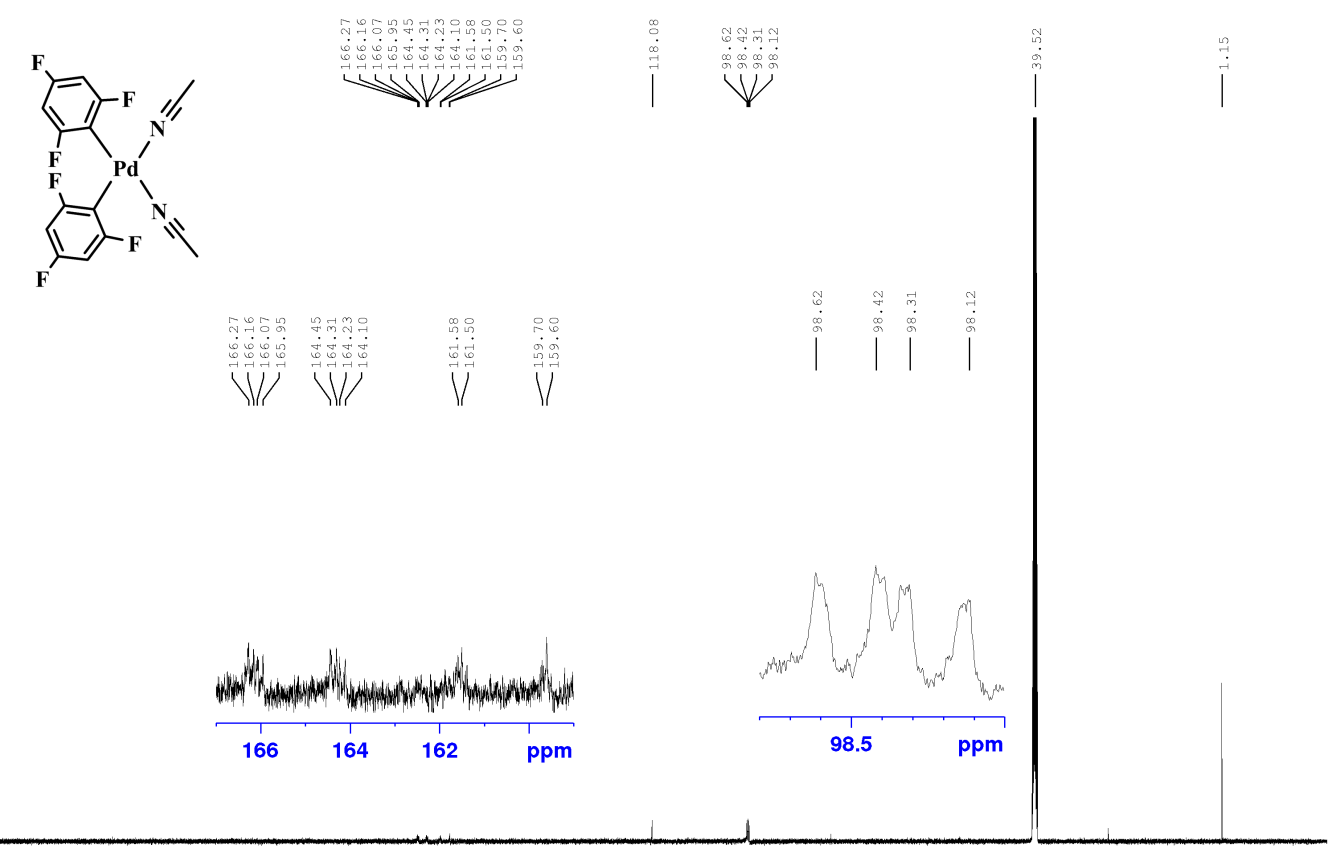

$240 \quad 220$

200

180

160

140

120

$100 \quad 80$

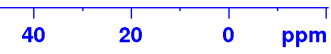


${ }^{13} \mathrm{C}\left\{{ }^{19} \mathrm{~F}\right\}$ NMR spectrum of $3 \mathrm{~b}\left(125 \mathrm{MHz}, \mathrm{DMSO}-d_{6}\right)$
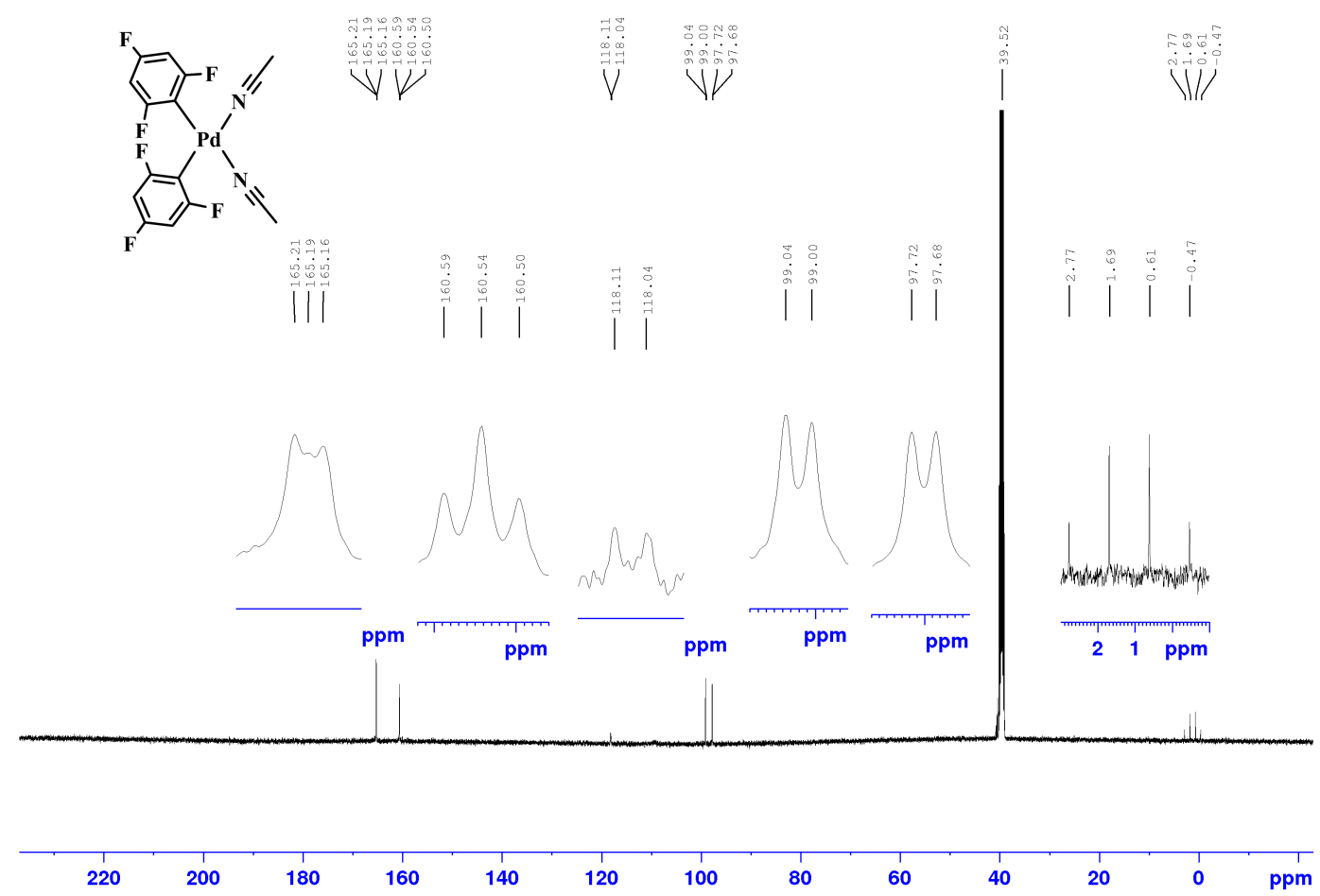

${ }^{13} \mathrm{C}\left\{{ }^{19} \mathrm{~F}\right\}$ NMR spectrum of $3 \mathrm{~b}\left(125 \mathrm{MHz}, \mathrm{CDCl}_{3}\right)$

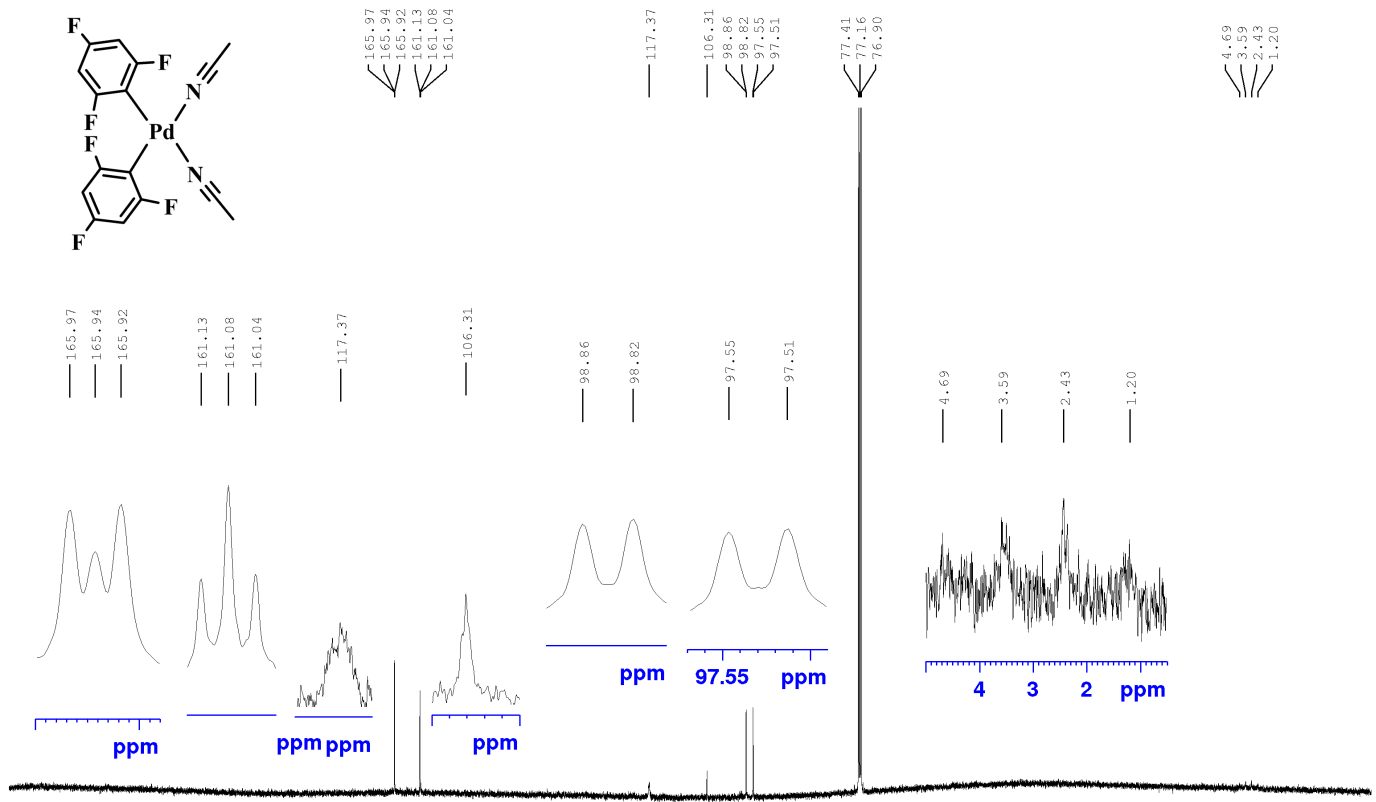

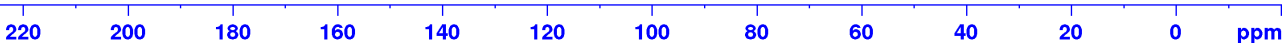


${ }^{19} \mathrm{~F}\left\{{ }^{1} \mathrm{H}\right\}$ NMR spectrum of $3 \mathrm{~b}\left(376 \mathrm{MHz}, \mathrm{CD}_{3} \mathrm{CN}\right)$

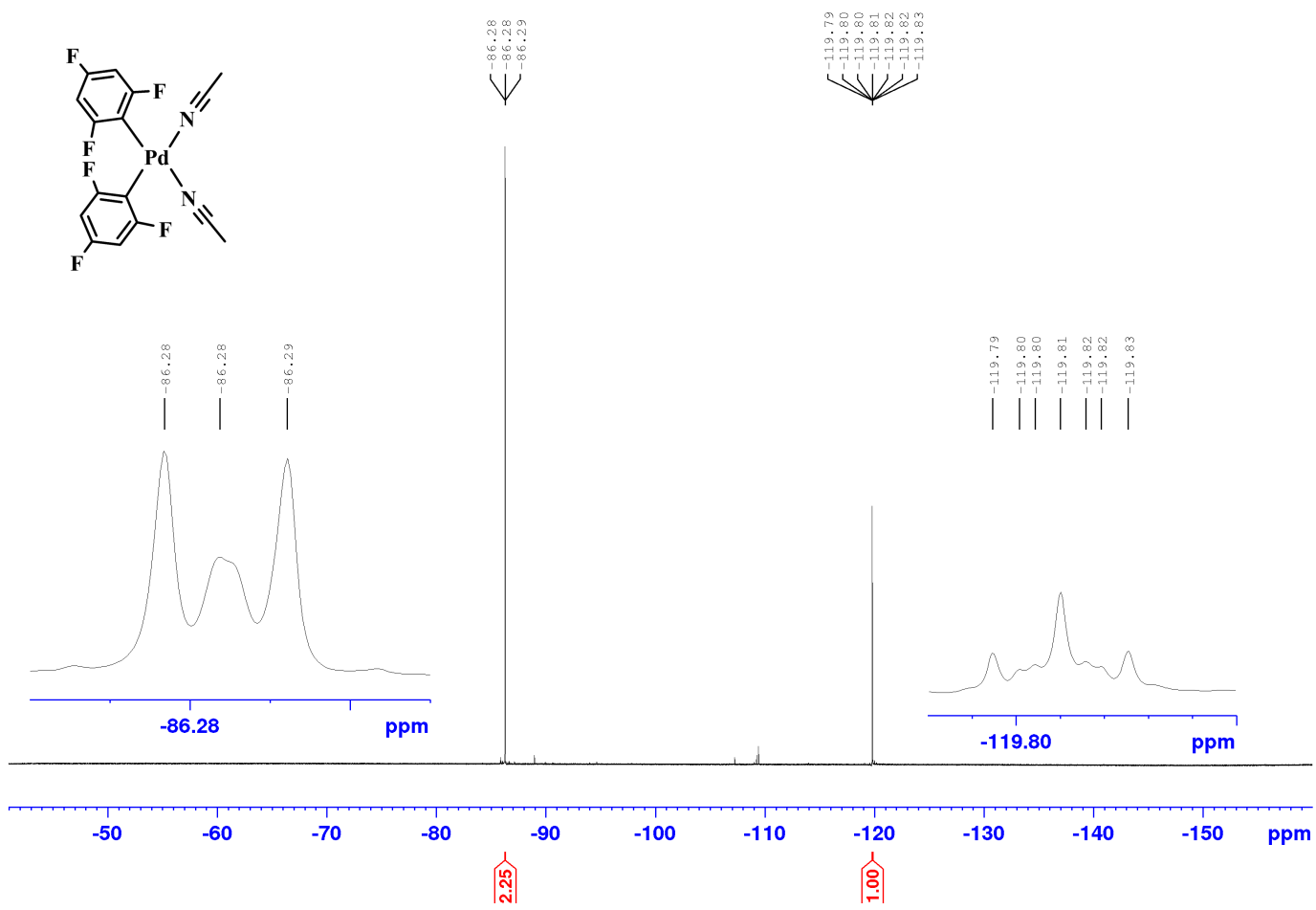

IR spectrum of $3 b$ in the solid-state

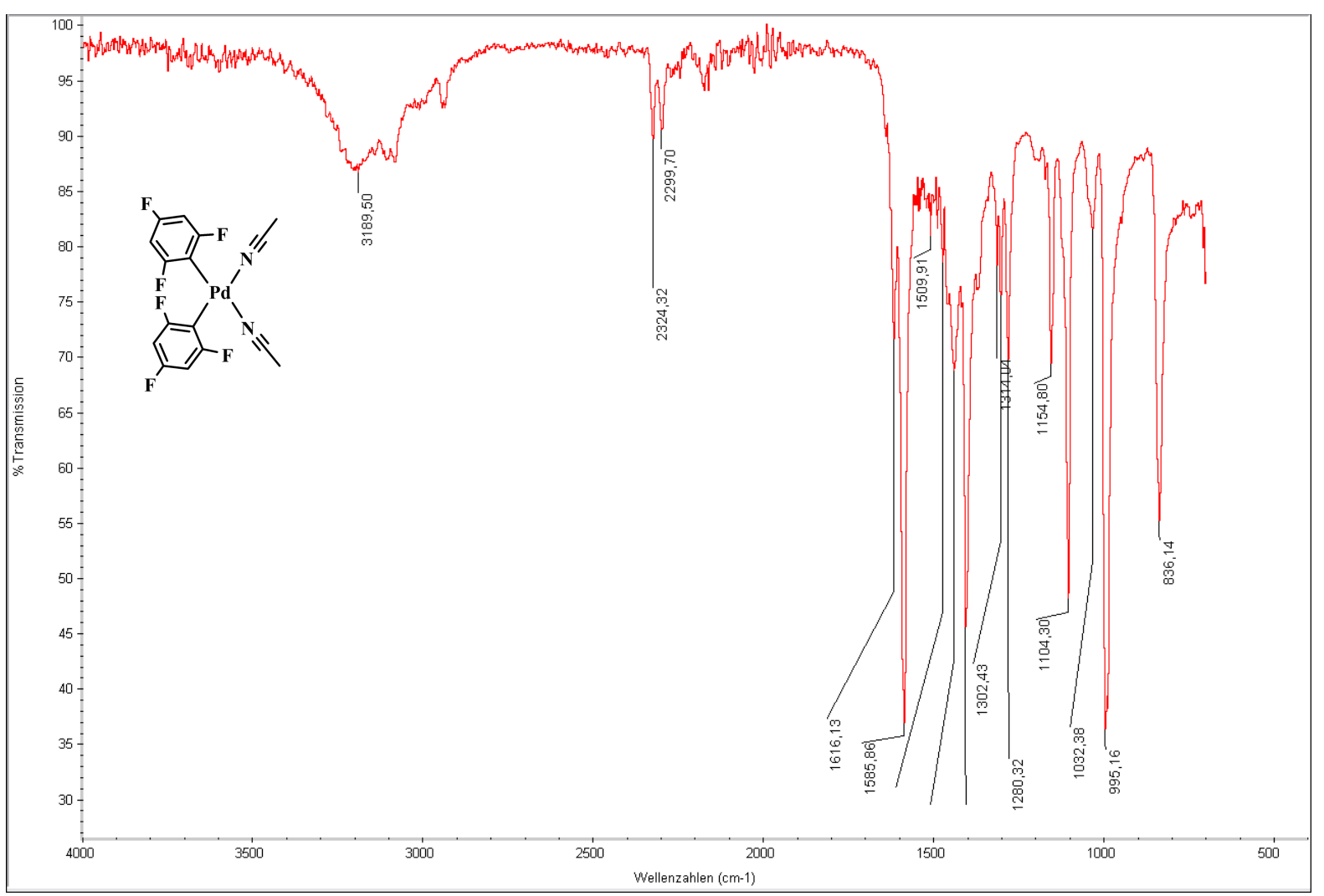


${ }^{1} \mathrm{H}$ NMR spectrum of $3 \mathrm{e}\left(300 \mathrm{MHz}\right.$, DMSO- $\left.d_{6}\right)$
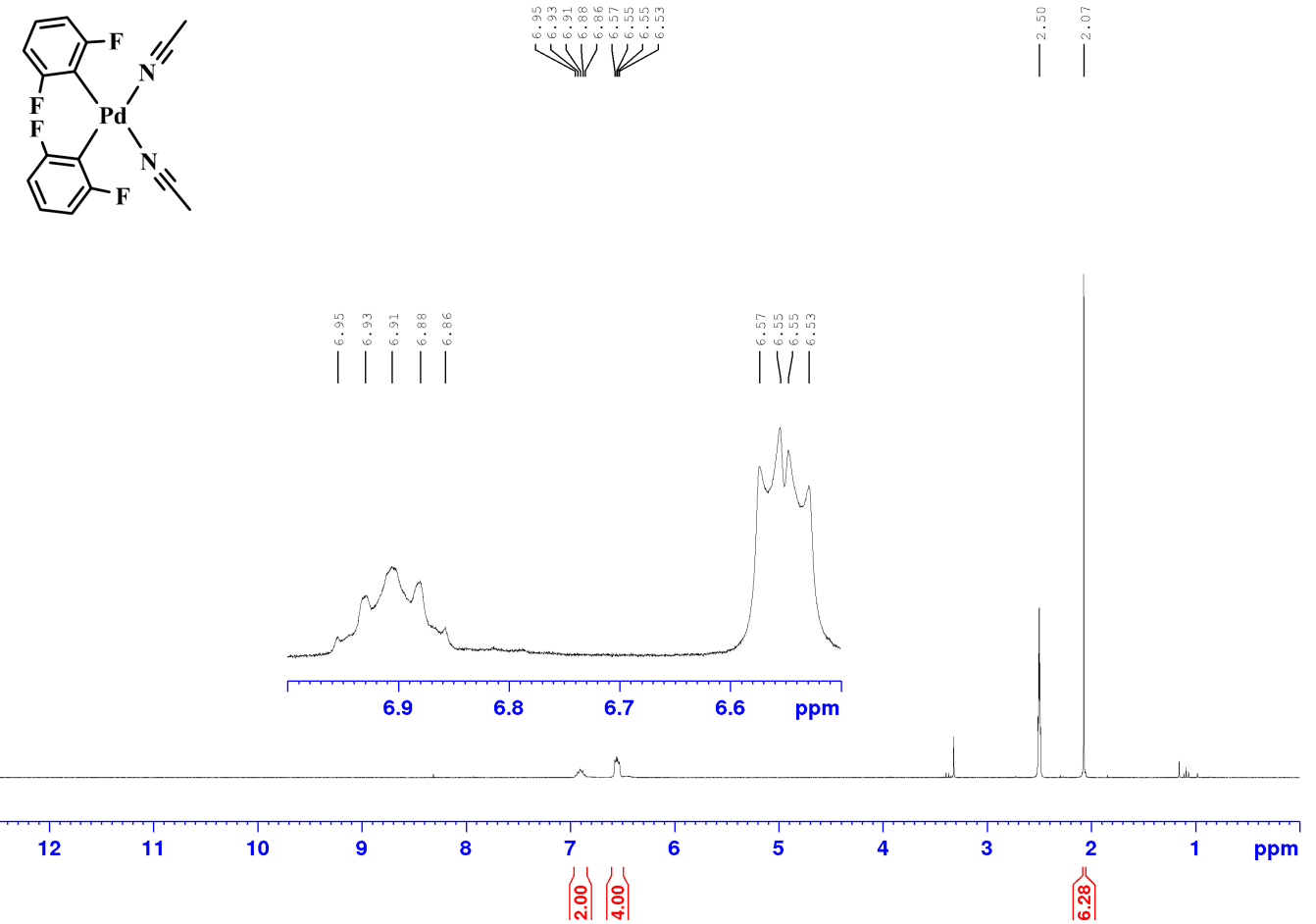

${ }^{13} \mathrm{C}\left\{{ }^{1} \mathrm{H}\right\}$ NMR spectrum of 3e (101 MHz, DMSO-d $\left.d_{6}\right)$

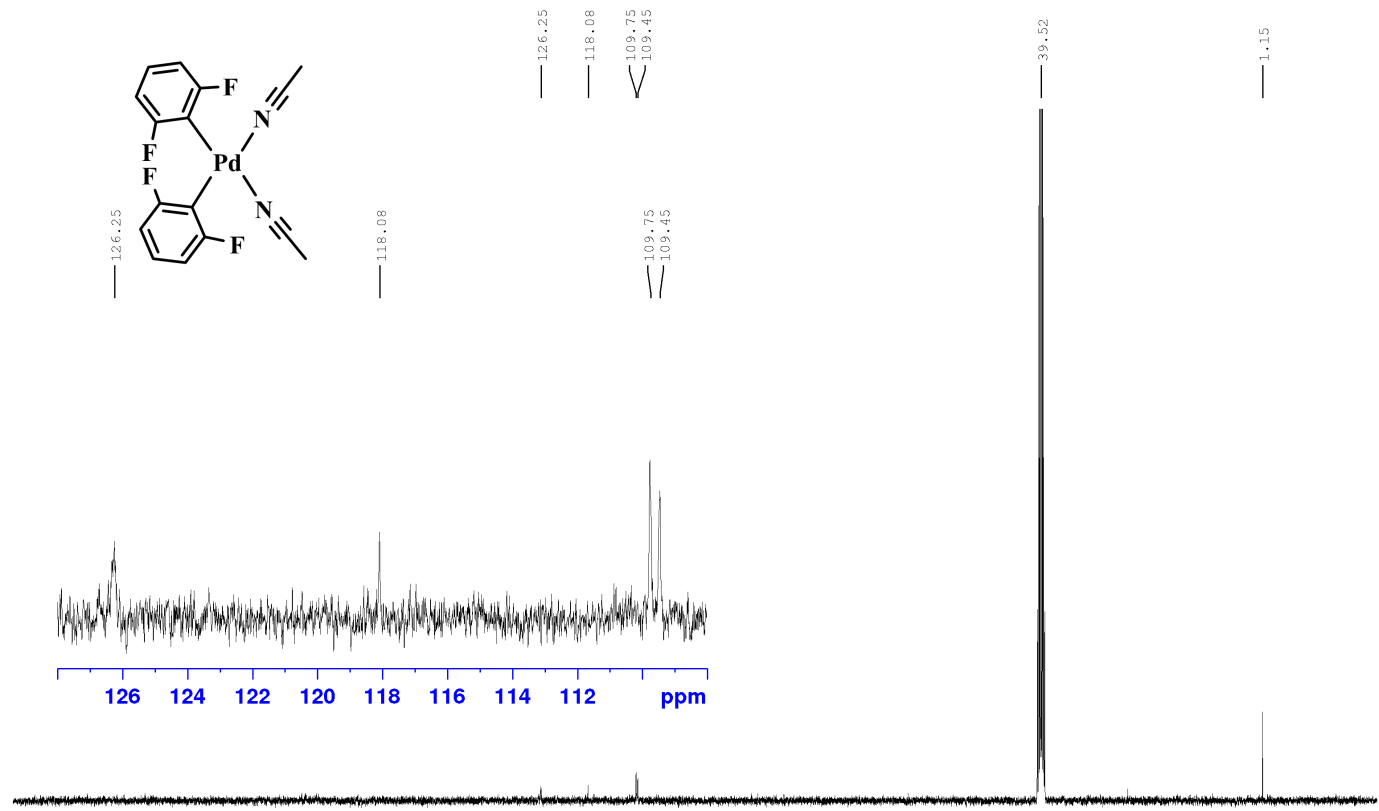

$\begin{array}{lllllllllllllllllllllll}210 & 200 & 190 & 180 & 170 & 160 & 150 & 140 & 130 & 120 & 110 & 100 & 90 & 80 & 70 & 60 & 50 & 40 & 30 & 20 & 10 & 0 & \mathrm{ppm}\end{array}$ 
${ }^{13} \mathrm{C}\left({ }^{1} \mathrm{H}\right\}$ NMR spectrum of $3 \mathrm{e}\left(101 \mathrm{MHz}, \mathrm{CD}_{3} \mathrm{CN}\right)$

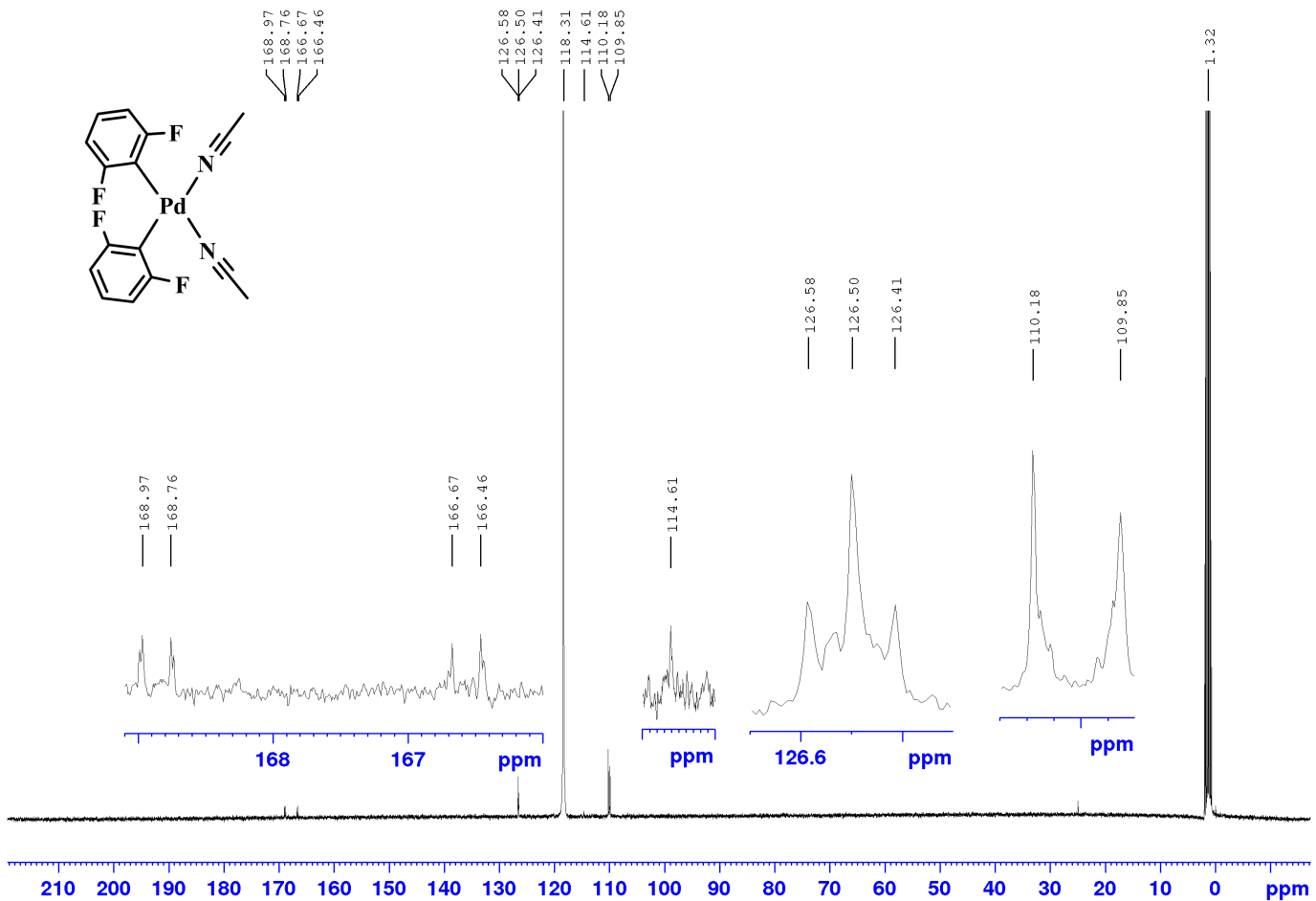

${ }^{13} \mathrm{C}\left\{{ }^{19} \mathrm{~F}\right\}$ NMR spectrum of $3 \mathrm{e}\left(126 \mathrm{MHz}, \mathrm{CD}_{3} \mathrm{CN}\right)$

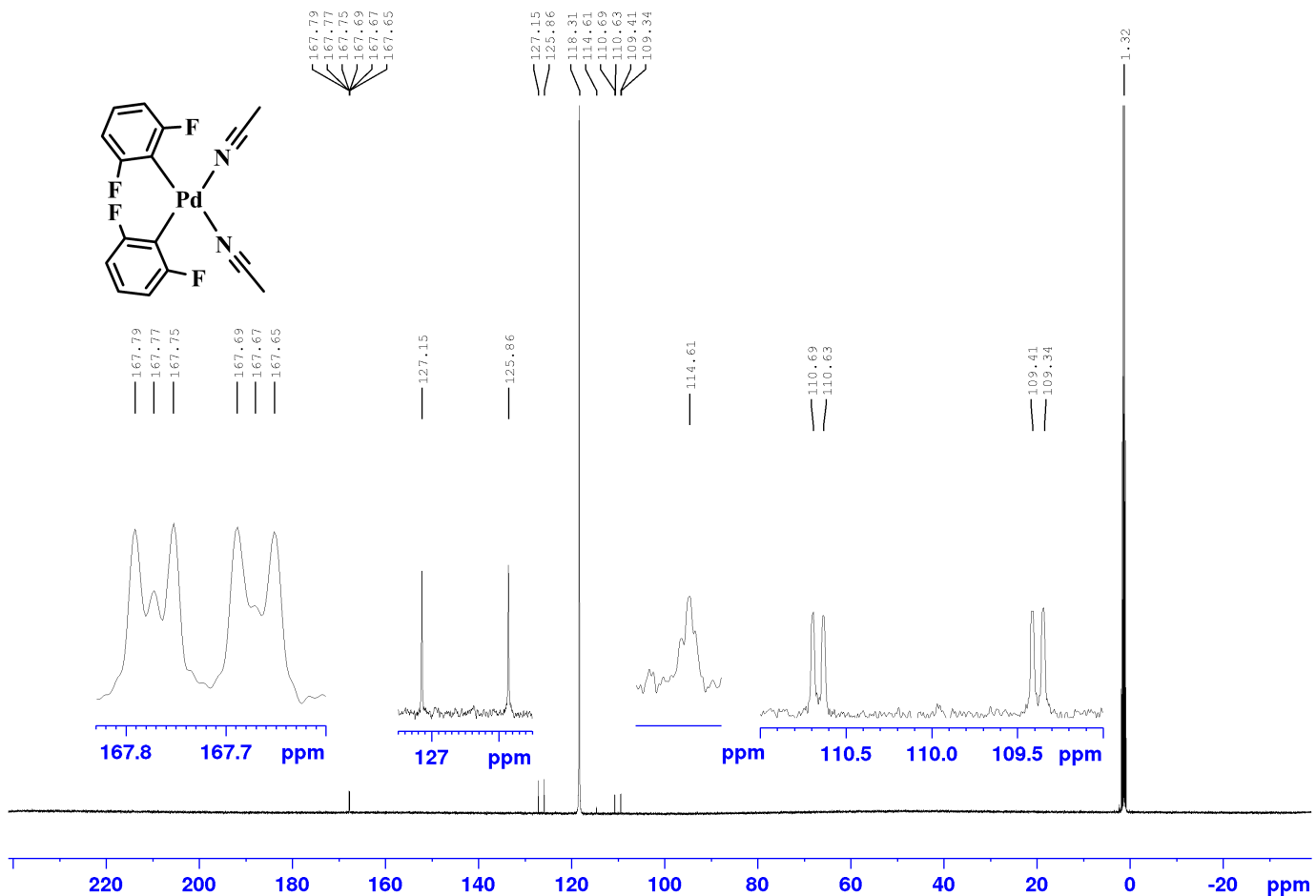


${ }^{19} \mathrm{~F}\left\{{ }^{1} \mathrm{H}\right\}$ NMR spectrum of $3 \mathrm{e}\left(376 \mathrm{MHz}, \mathrm{CD}_{3} \mathrm{CN}\right)$

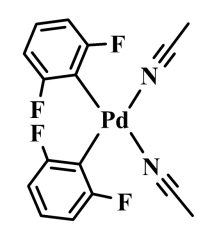

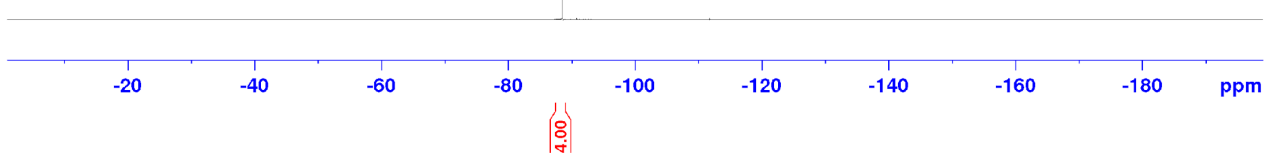

IR spectrum of $3 \mathrm{e}$ in the solid-state

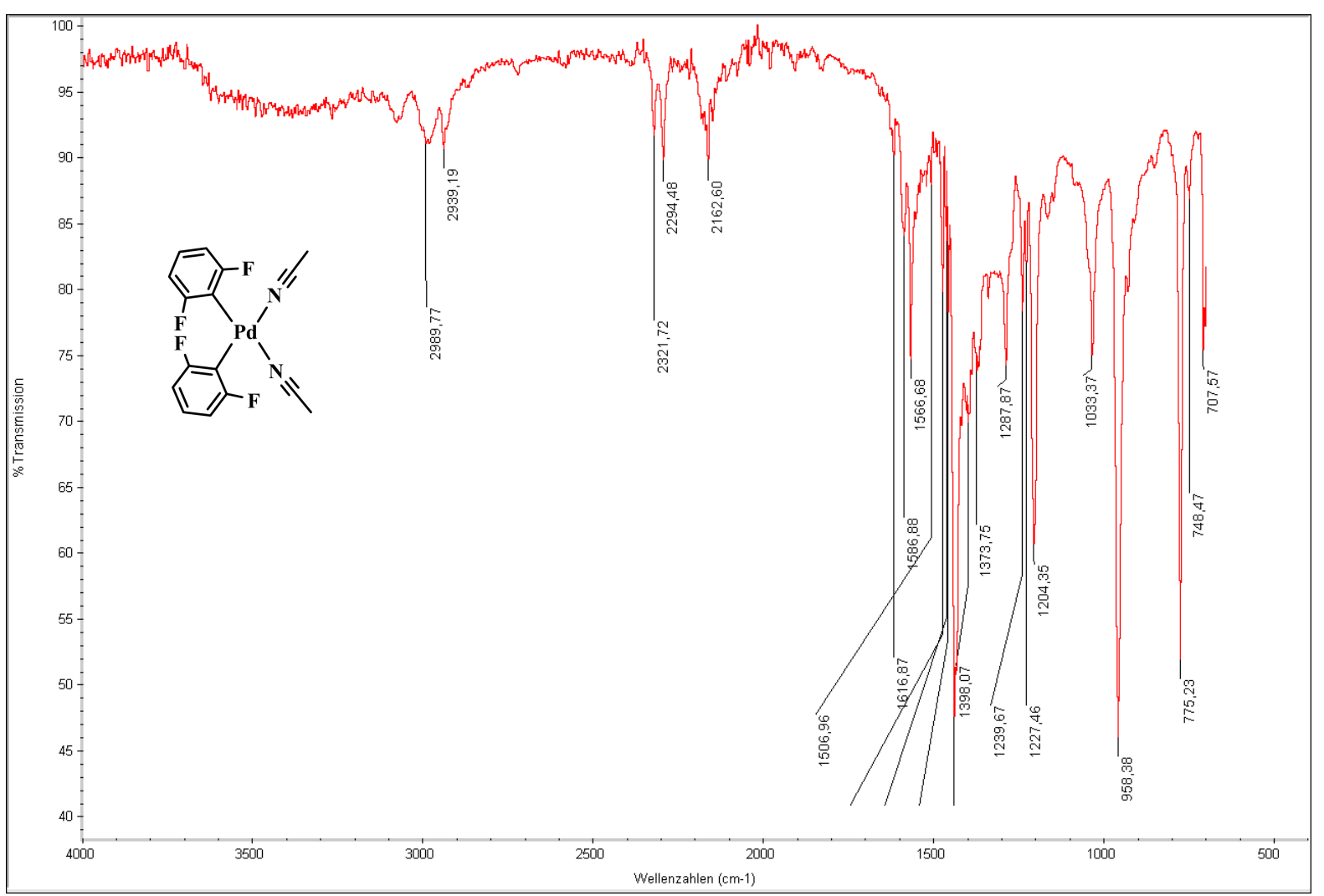


${ }^{13} \mathrm{C}\left\{{ }^{19} \mathrm{~F}\right\}$ NMR spectrum of $2 \mathrm{a}\left(125 \mathrm{MHz}, \mathrm{CDCl}_{3}\right)$

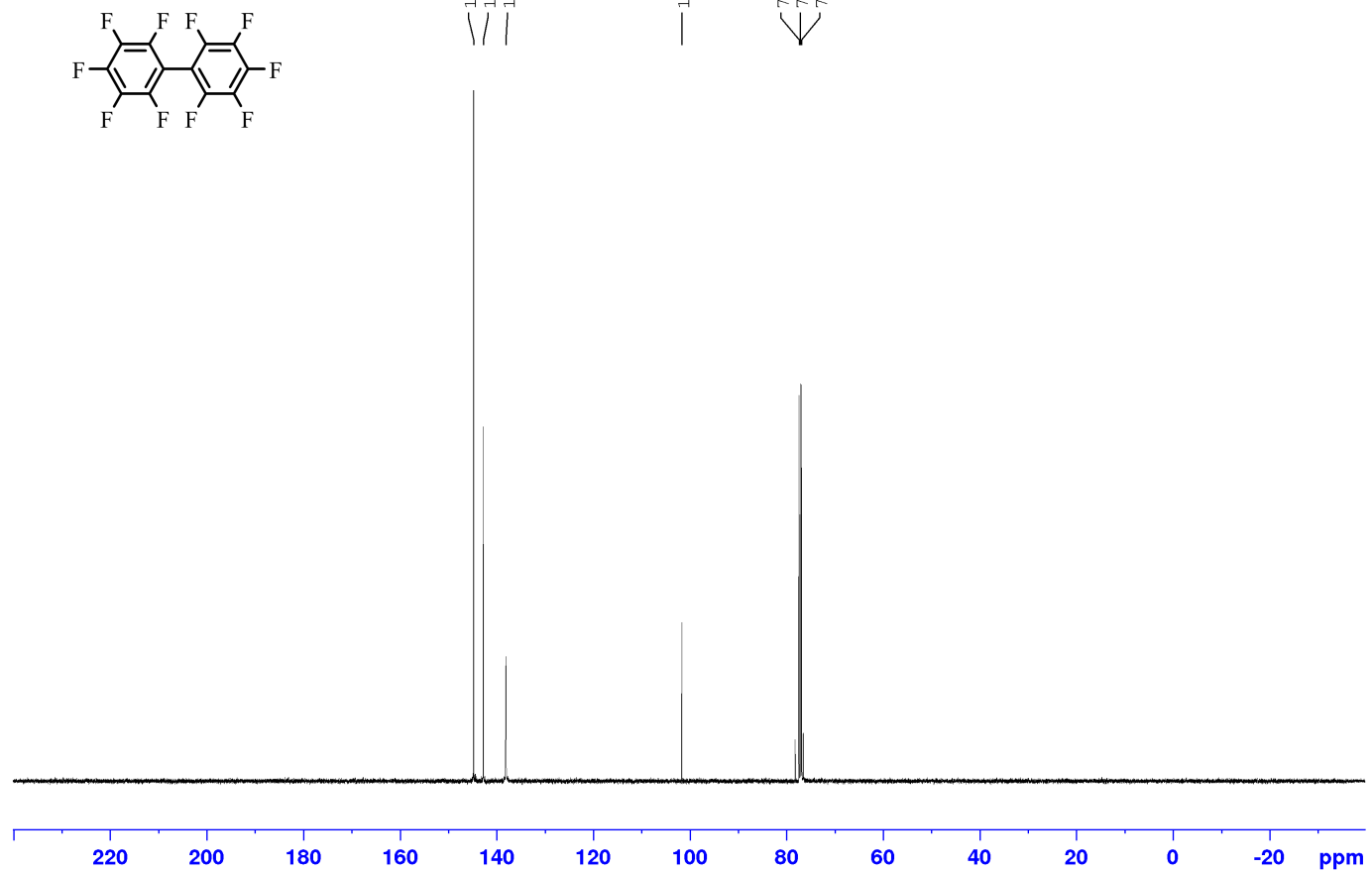

${ }^{19} \mathrm{~F}\left\{{ }^{1} \mathrm{H}\right\}$ NMR spectrum of $2 \mathrm{a}\left(376 \mathrm{MHz}, \mathrm{CDCl}_{3}\right)$

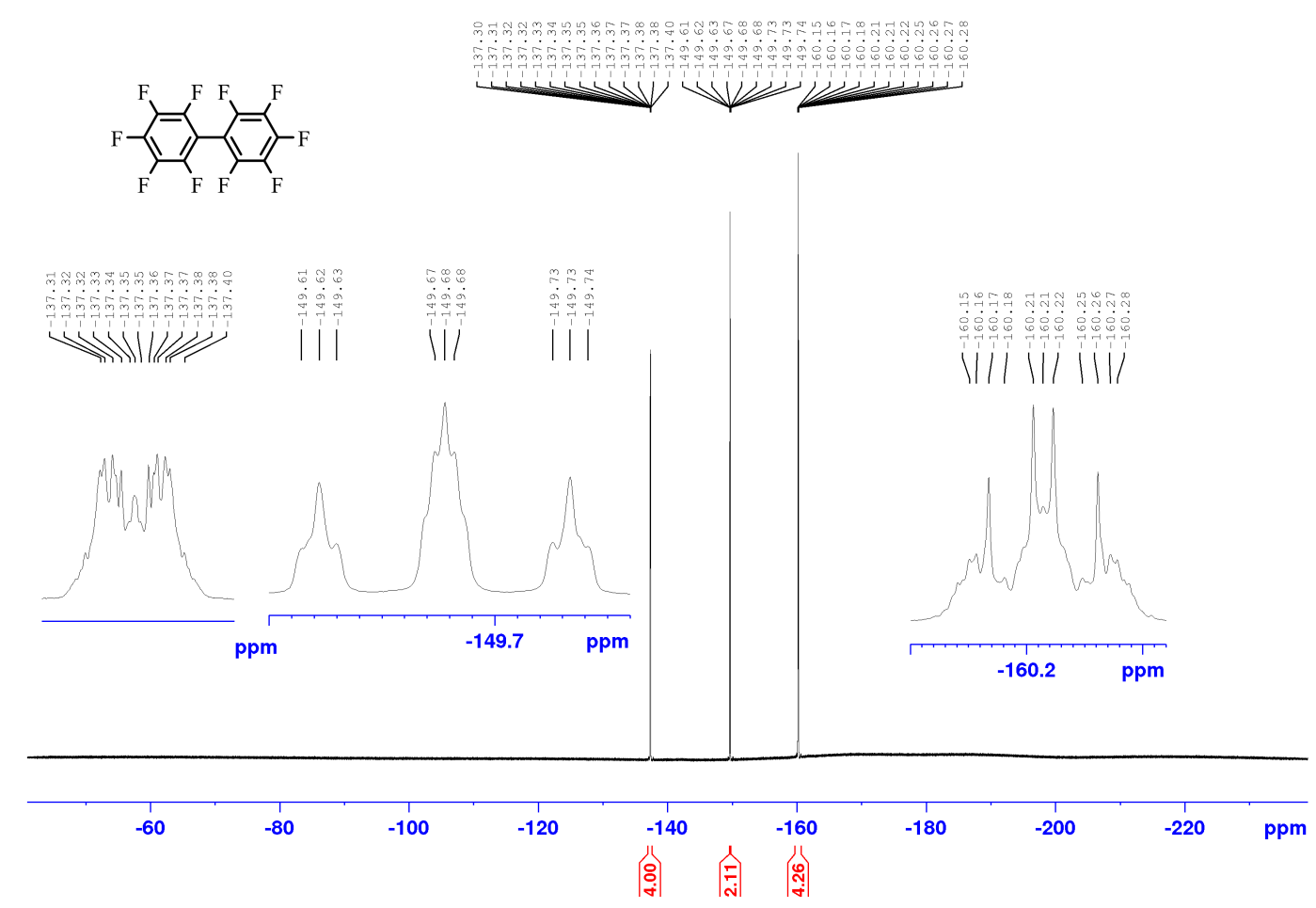




\section{GC-MS of 2a}

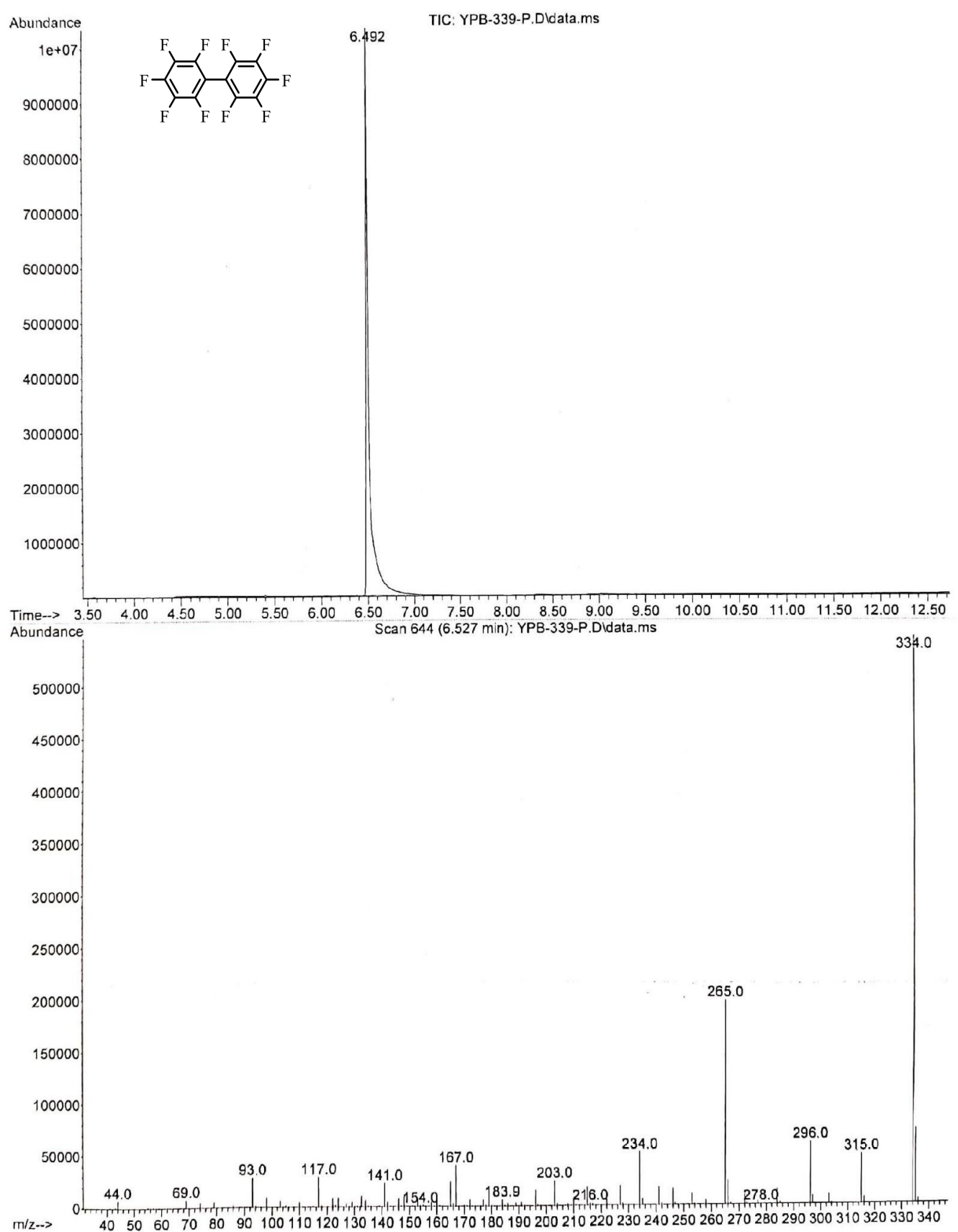


${ }^{1} \mathrm{H}$ NMR spectrum of $2 \mathrm{~b}\left(300 \mathrm{MHz}, \mathrm{C}_{6} \mathrm{D}_{6}\right)$

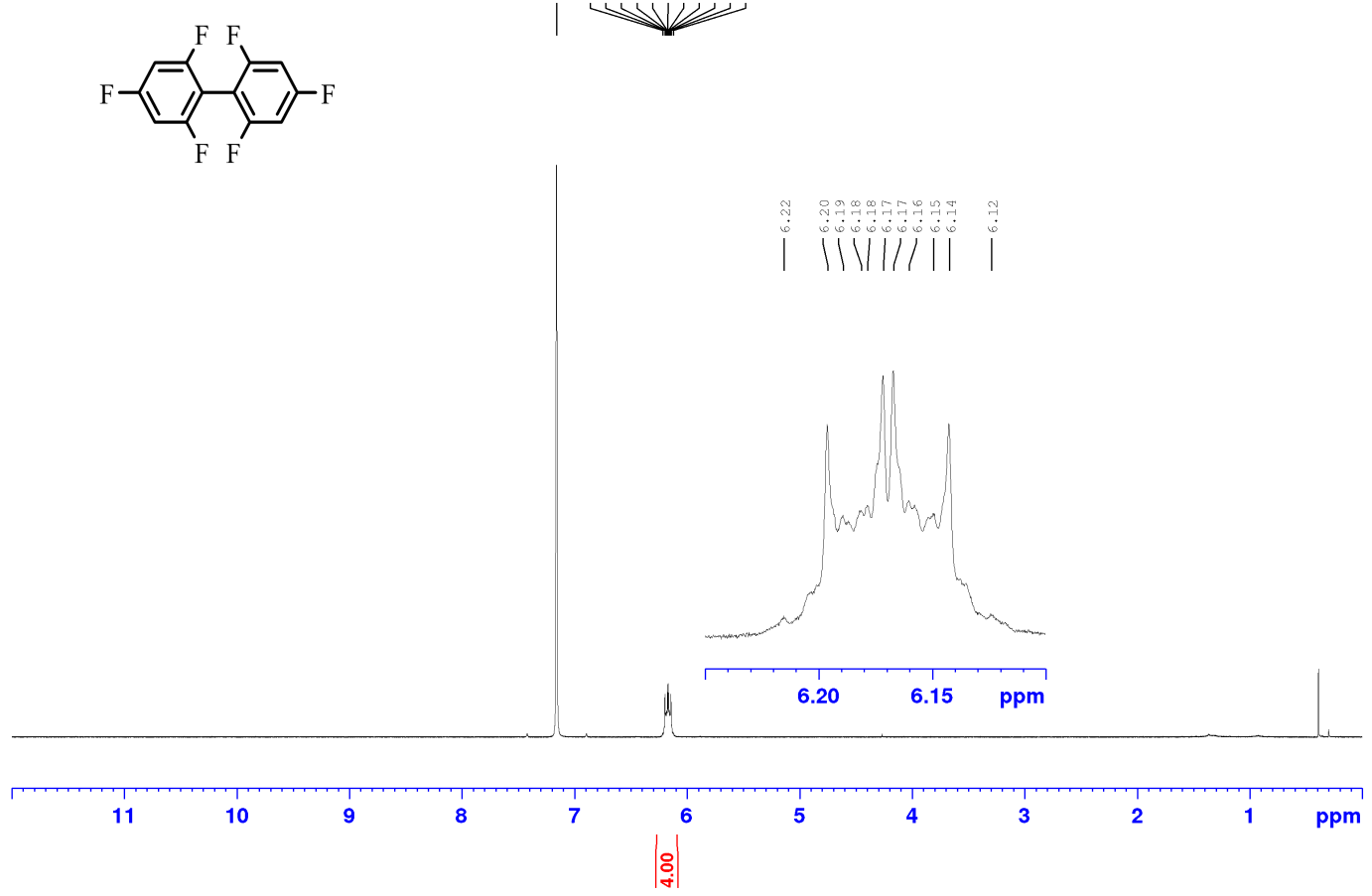

${ }^{13} \mathrm{C}\left\{{ }^{1} \mathrm{H}\right\}$ NMR spectrum of $2 \mathrm{~b}\left(125 \mathrm{MHz}, \mathrm{CDCl}_{3}\right)$

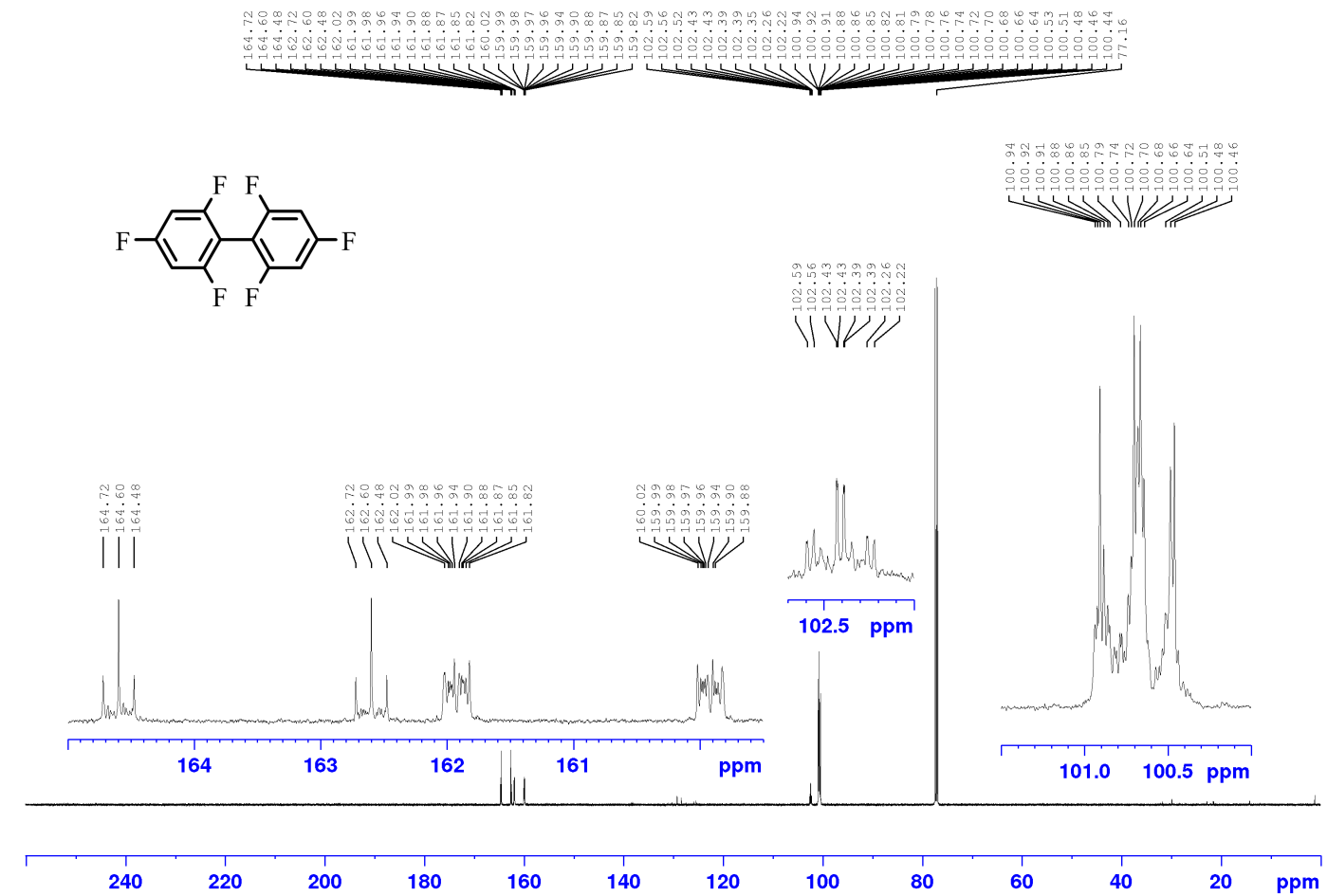


${ }^{13} \mathrm{C}\left\{{ }^{19} \mathrm{~F}\right\}$ NMR spectrum of $2 \mathrm{~b}\left(125 \mathrm{MHz}, \mathrm{CDCl}_{3}\right)$<smiles>Fc1cc(F)c(-c2c(F)cc(F)cc2F)c(F)c1</smiles>
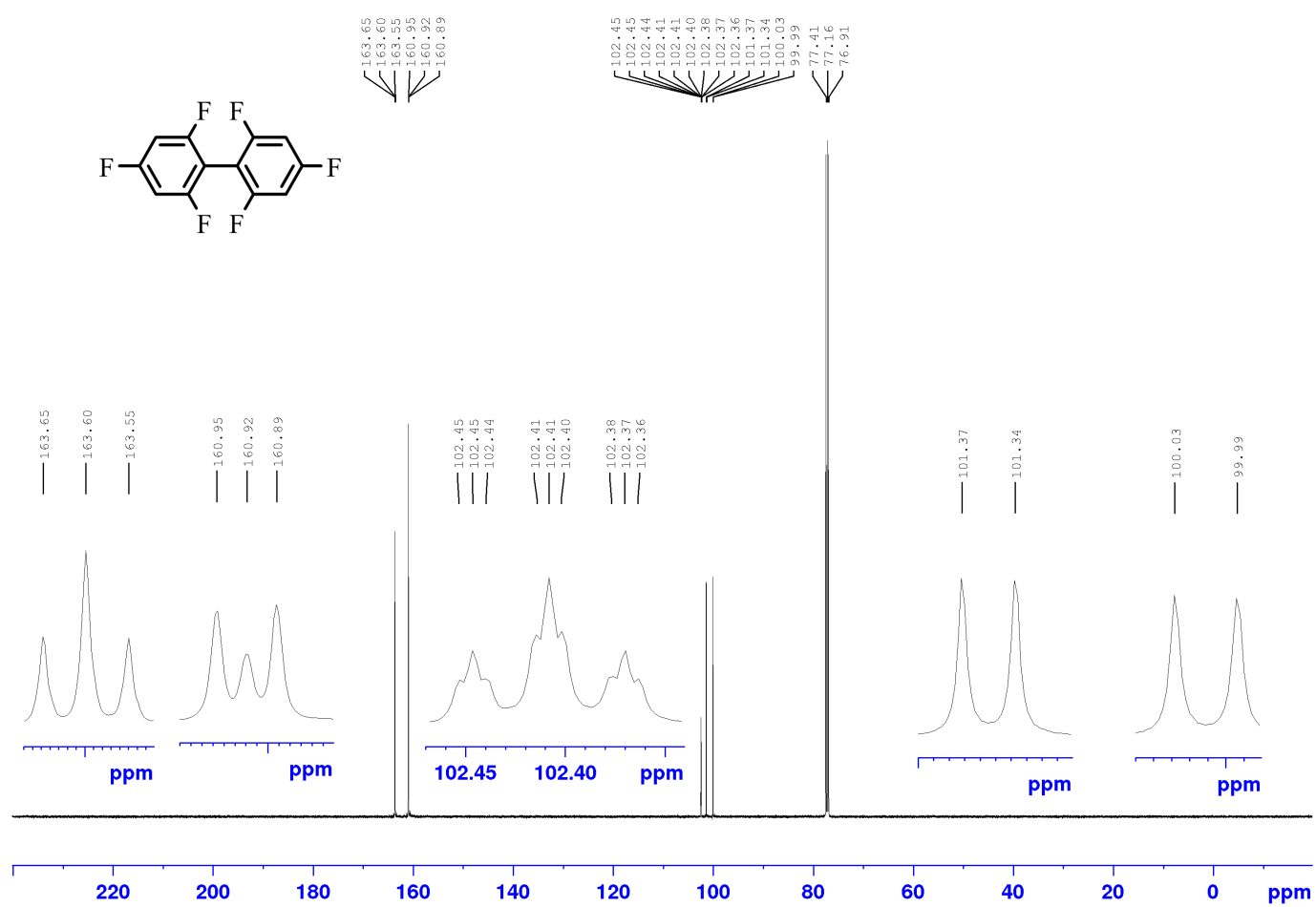

${ }^{19} \mathrm{~F}\left\{{ }^{1} \mathrm{H}\right\}$ NMR spectrum of $2 \mathrm{~b}\left(376 \mathrm{MHz}, \mathrm{CDCl}_{3}\right)$

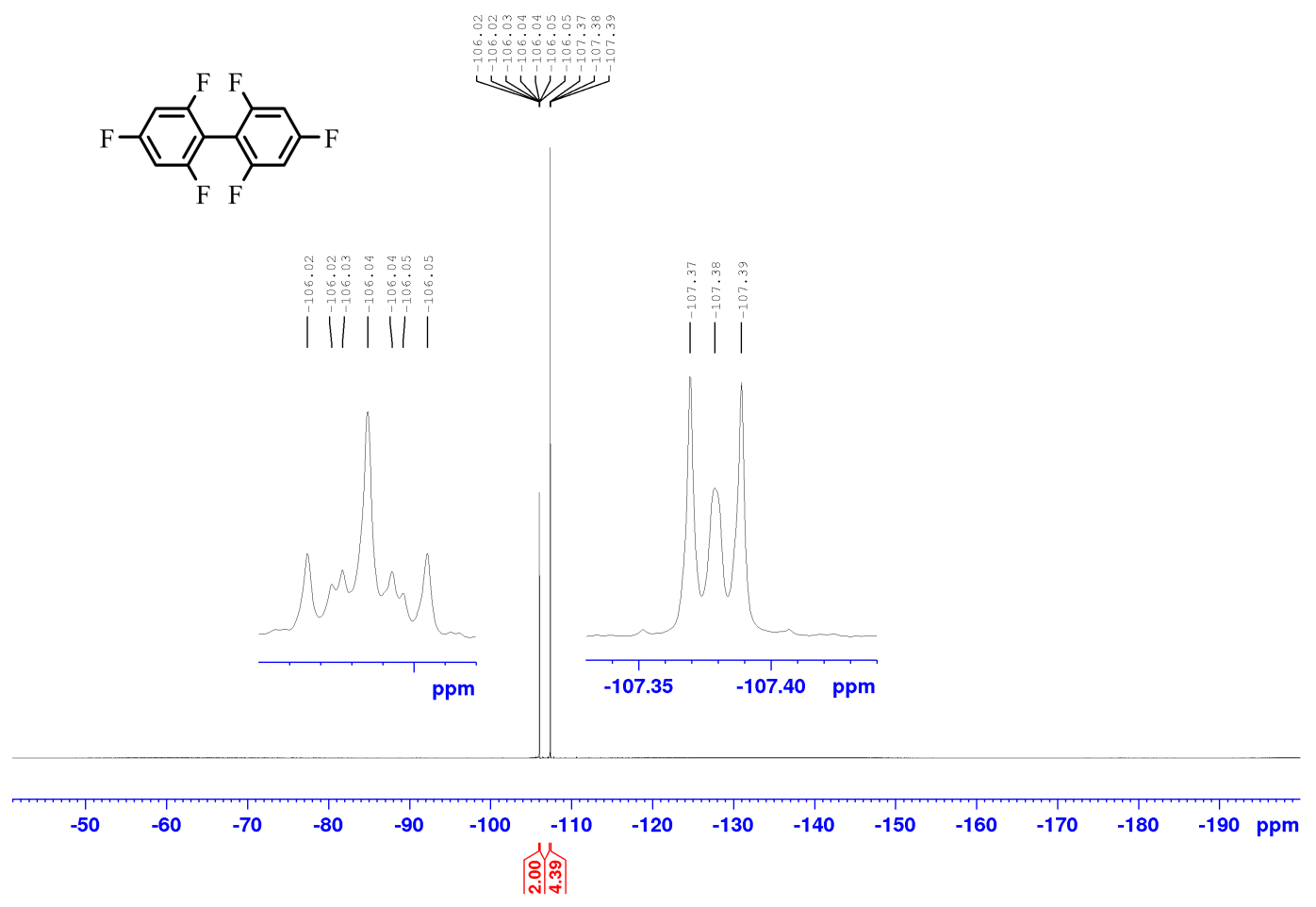




\section{GC-MS of $2 b$}

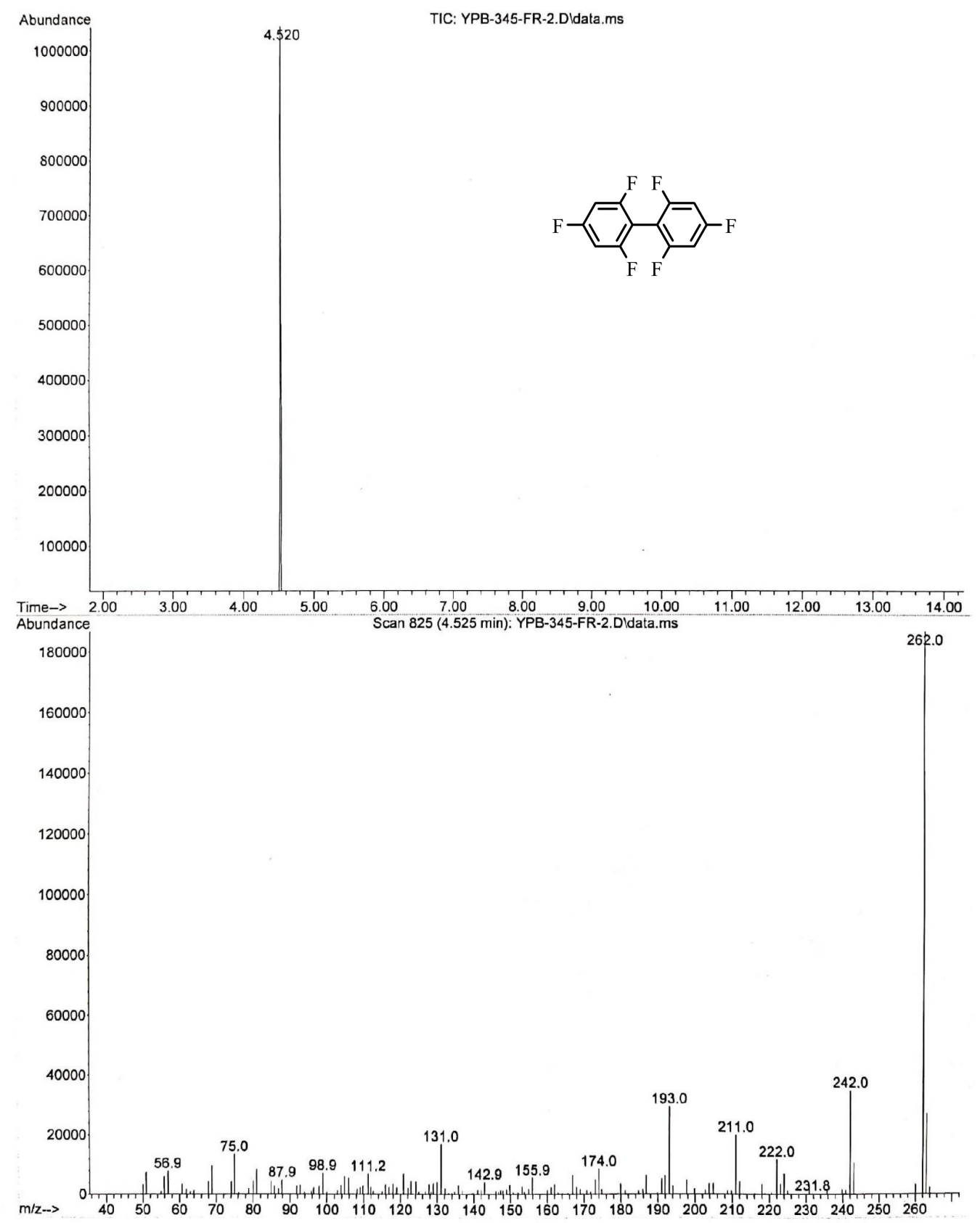


${ }^{1} \mathrm{H}$ NMR spectrum of $2 \mathrm{c}\left(500 \mathrm{MHz}, \mathrm{CDCl}_{3}\right)$<smiles>Fc1cc(F)c(-c2c(F)cc(F)c(F)c2F)c(F)c1F</smiles>
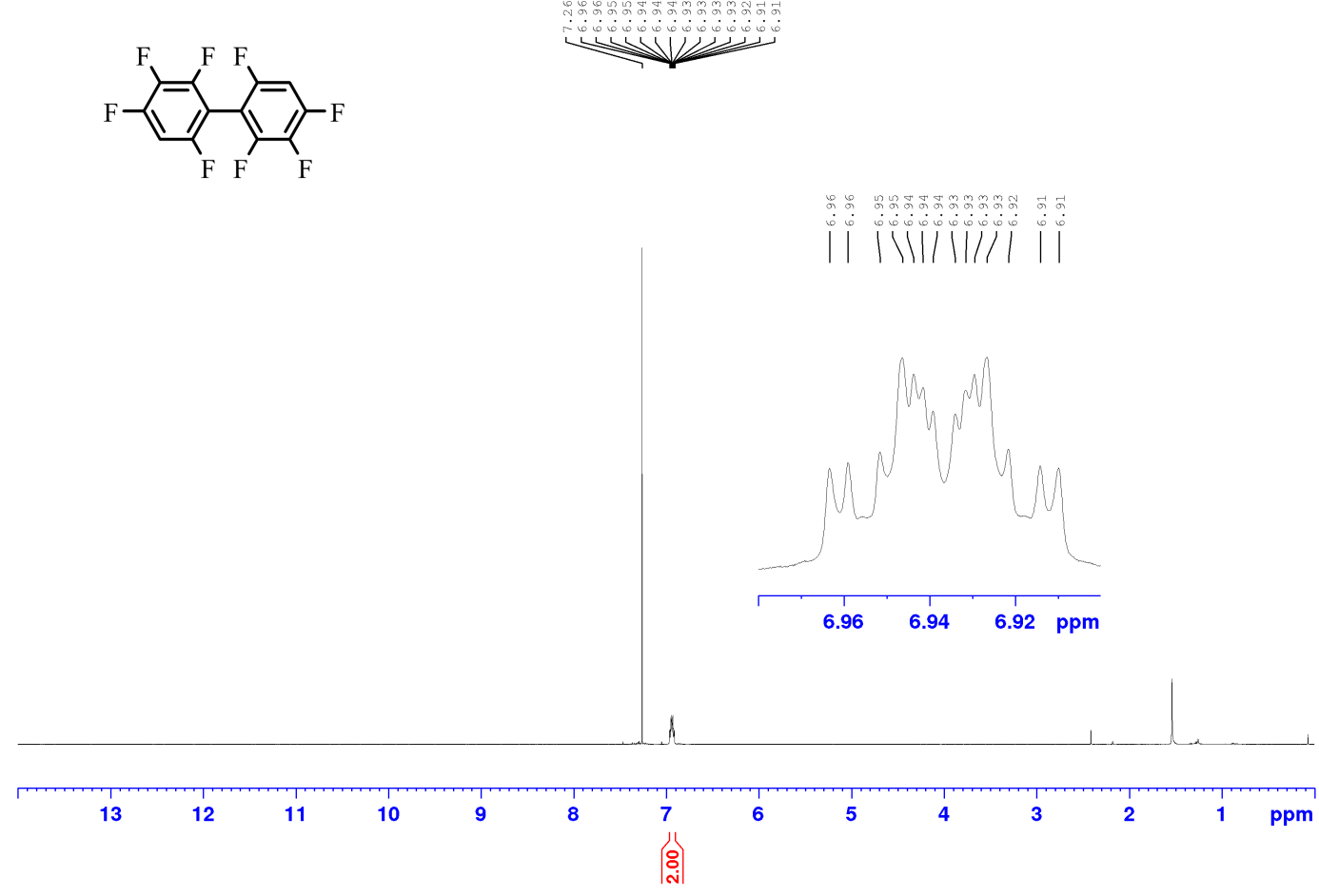

${ }^{13} \mathrm{C}\left\{{ }^{1} \mathrm{H}\right\}$ NMR spectrum of $2 \mathrm{c}\left(125 \mathrm{MHz}, \mathrm{CDCl}_{3}\right)$<smiles>Fc1cc(F)c(-c2c(F)cc(F)c(F)c2F)c(F)c1F</smiles>
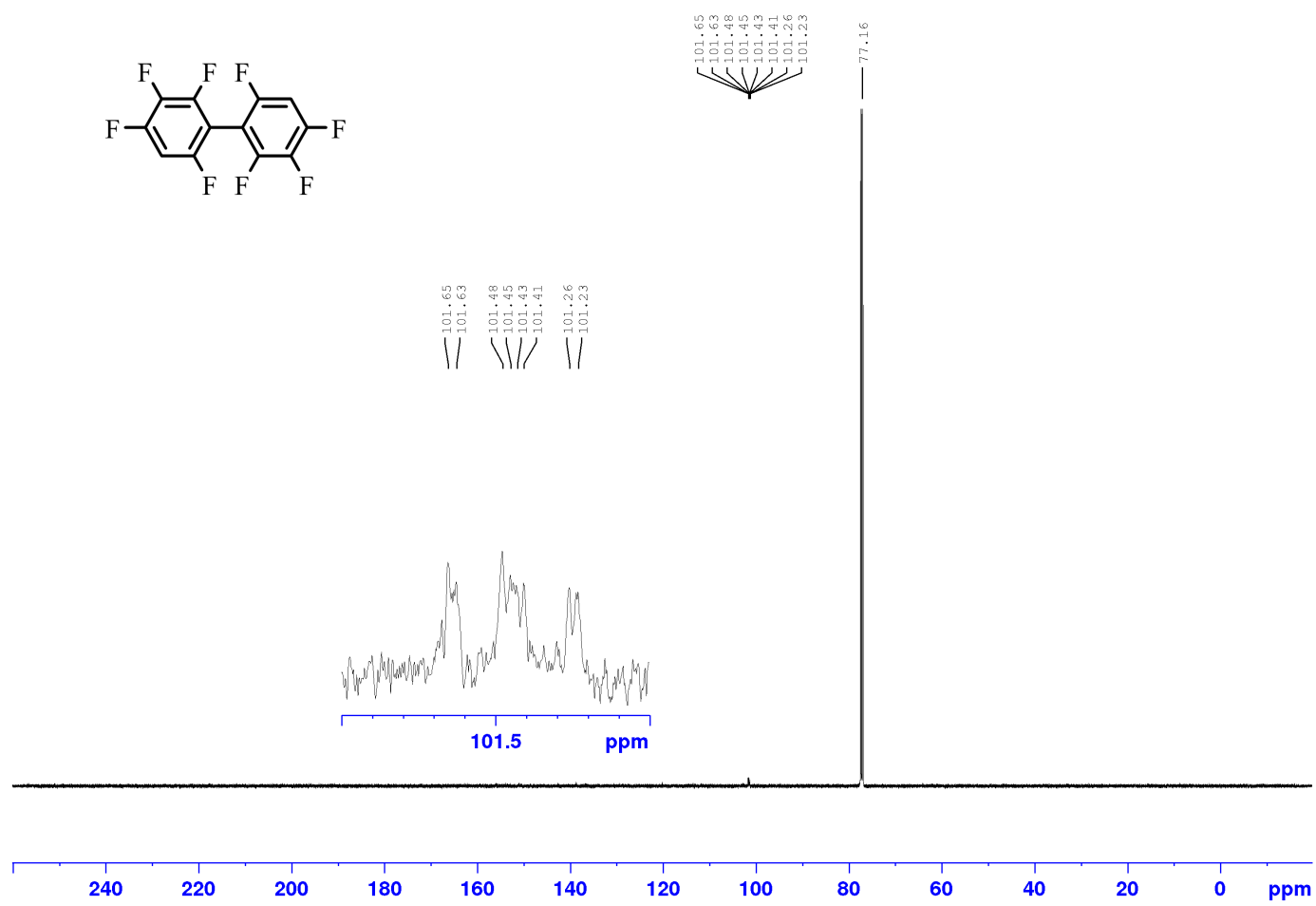
${ }^{13} \mathrm{C}\left\{{ }^{19} \mathrm{~F}\right\}$ NMR spectrum of $2 \mathrm{c}\left(125 \mathrm{MHz}, \mathrm{CDCl}_{3}\right)$
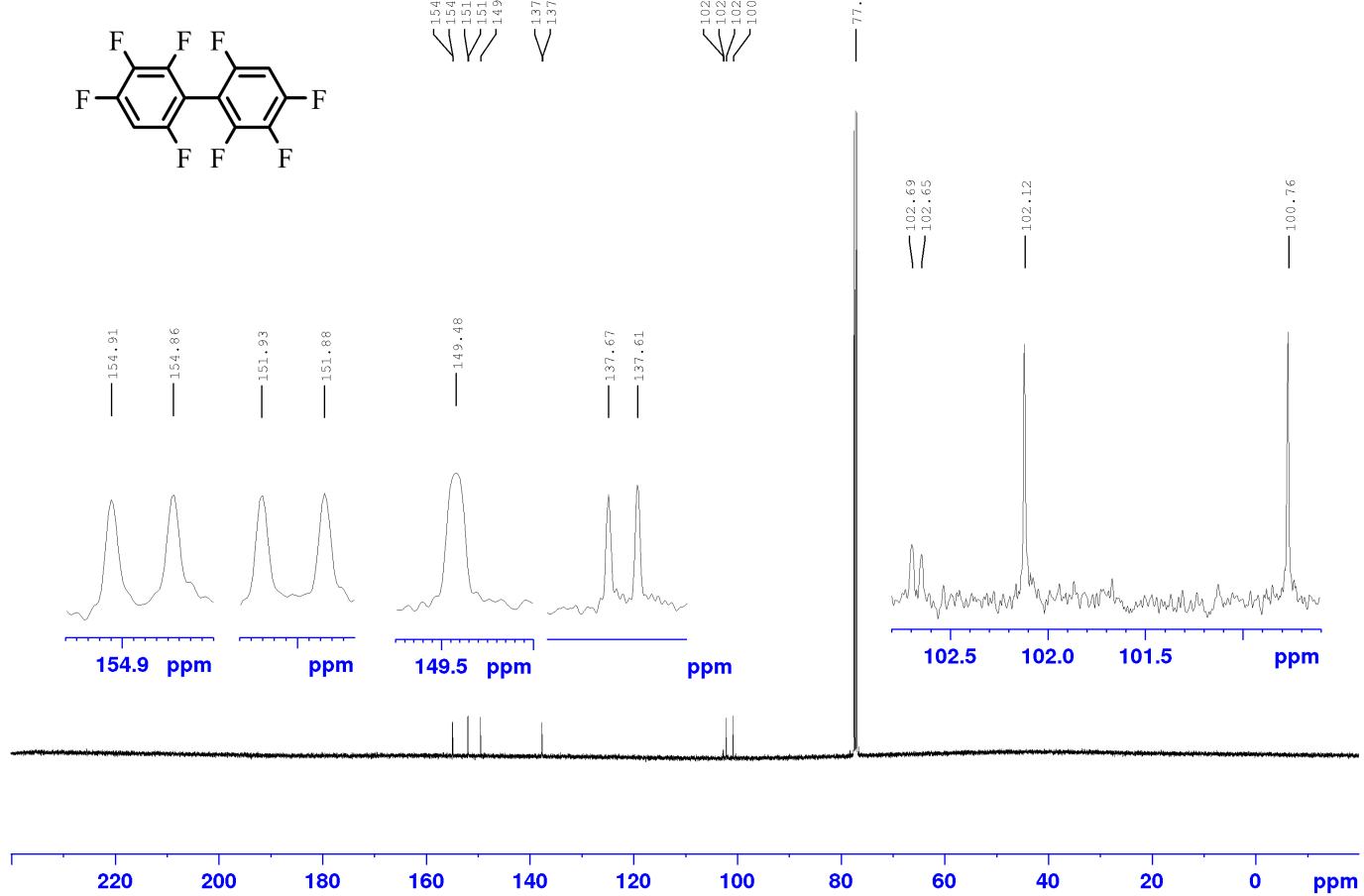

${ }^{19} \mathrm{~F}\left\{{ }^{1} \mathrm{H}\right\}$ NMR spectrum of $2 \mathrm{c}\left(376 \mathrm{MHz}, \mathrm{CDCl}_{3}\right)$

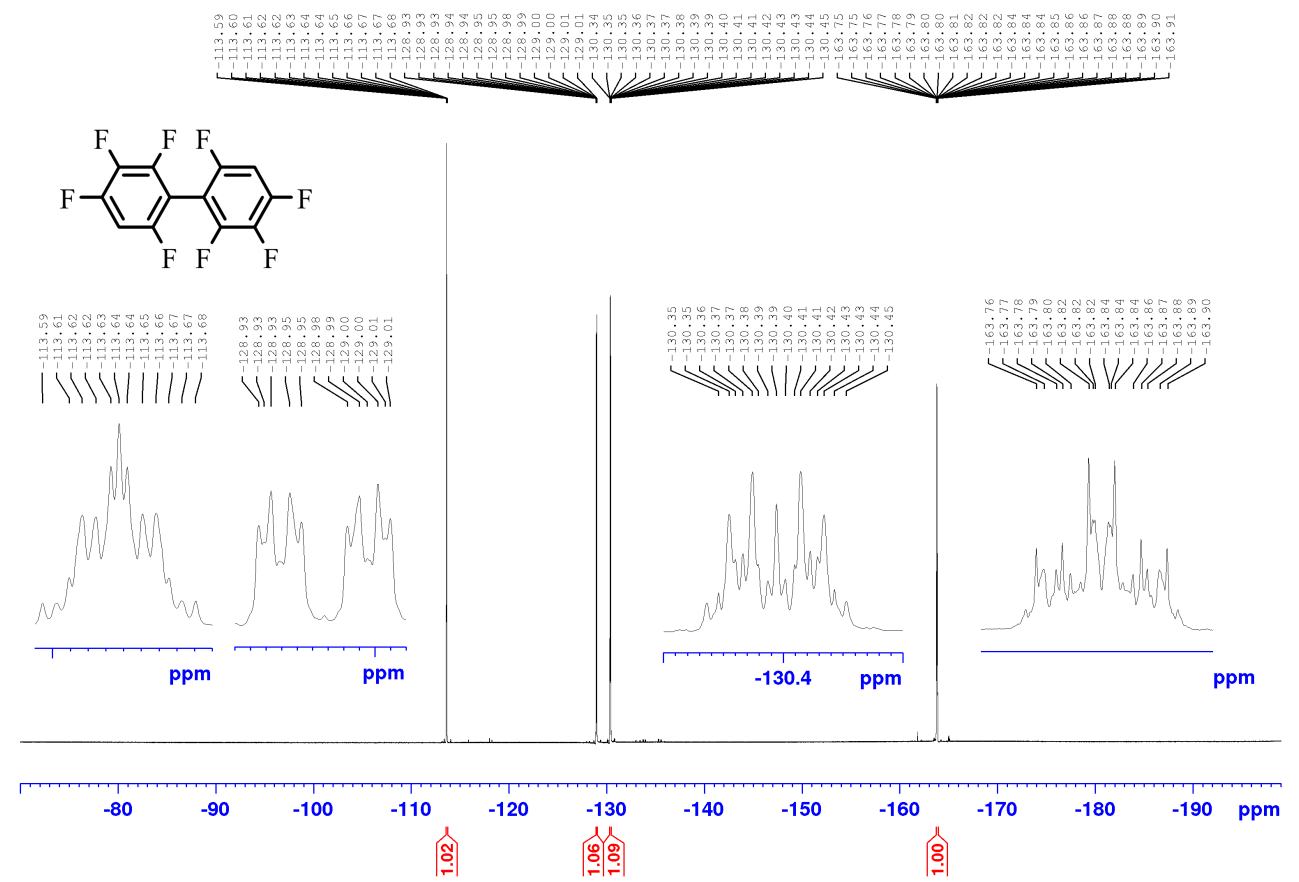




\section{GC-MS of 2c}

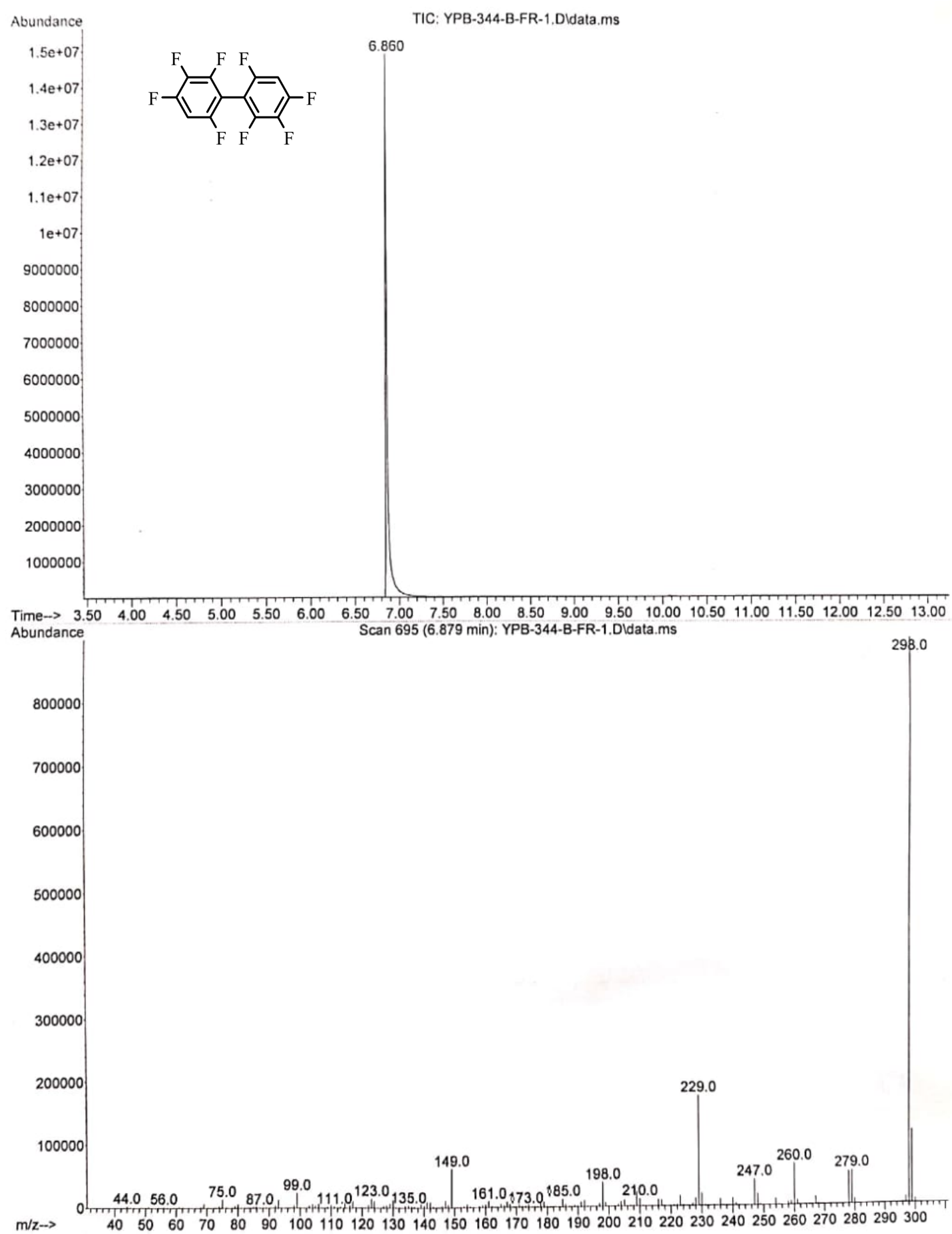


${ }^{1} \mathrm{H}$ NMR spectrum of $2 \mathrm{~d}\left(500 \mathrm{MHz}, \mathrm{C}_{6} \mathrm{D}_{6}\right)$

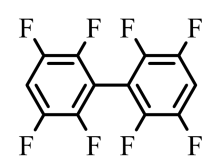

$\underbrace{0}$

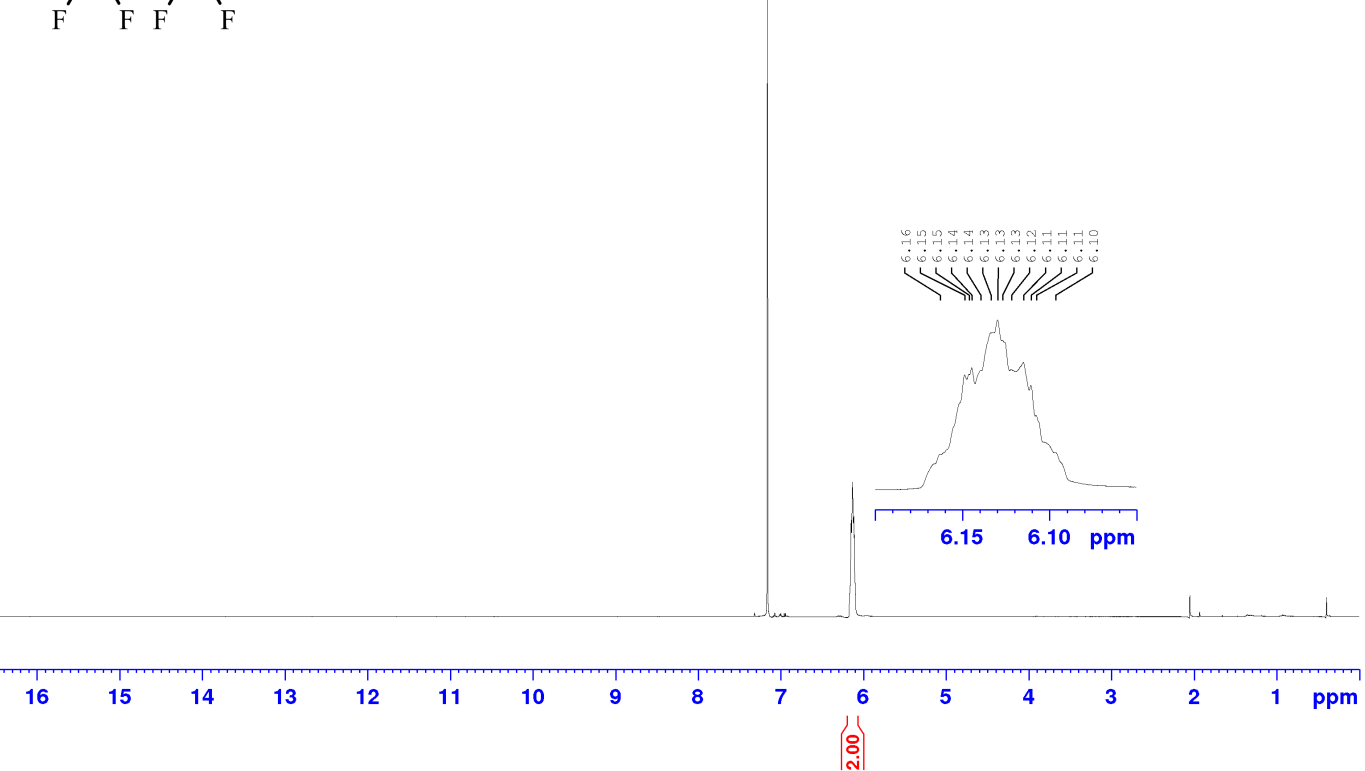

${ }^{13} \mathrm{C}\left\{{ }^{1} \mathrm{H}\right\}$ NMR spectrum of $2 \mathrm{~d}\left(125 \mathrm{MHz}, \mathrm{C}_{6} \mathrm{D}_{6}\right)$

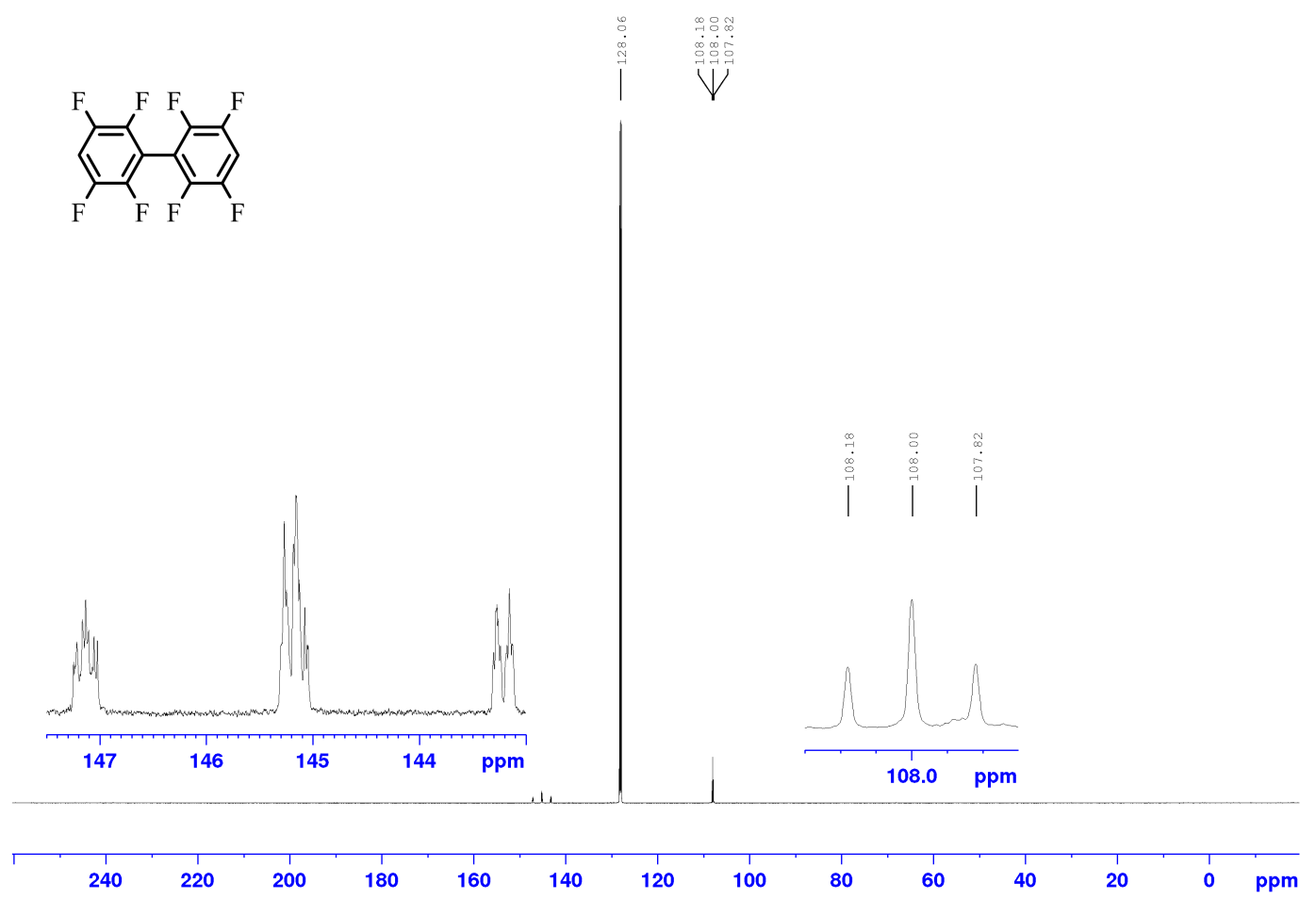


${ }^{13} \mathrm{C}\left\{{ }^{19} \mathrm{~F}\right\}$ NMR spectrum of $2 \mathrm{~d}\left(125 \mathrm{MHz}, \mathrm{C}_{6} \mathrm{D}_{6}\right)$

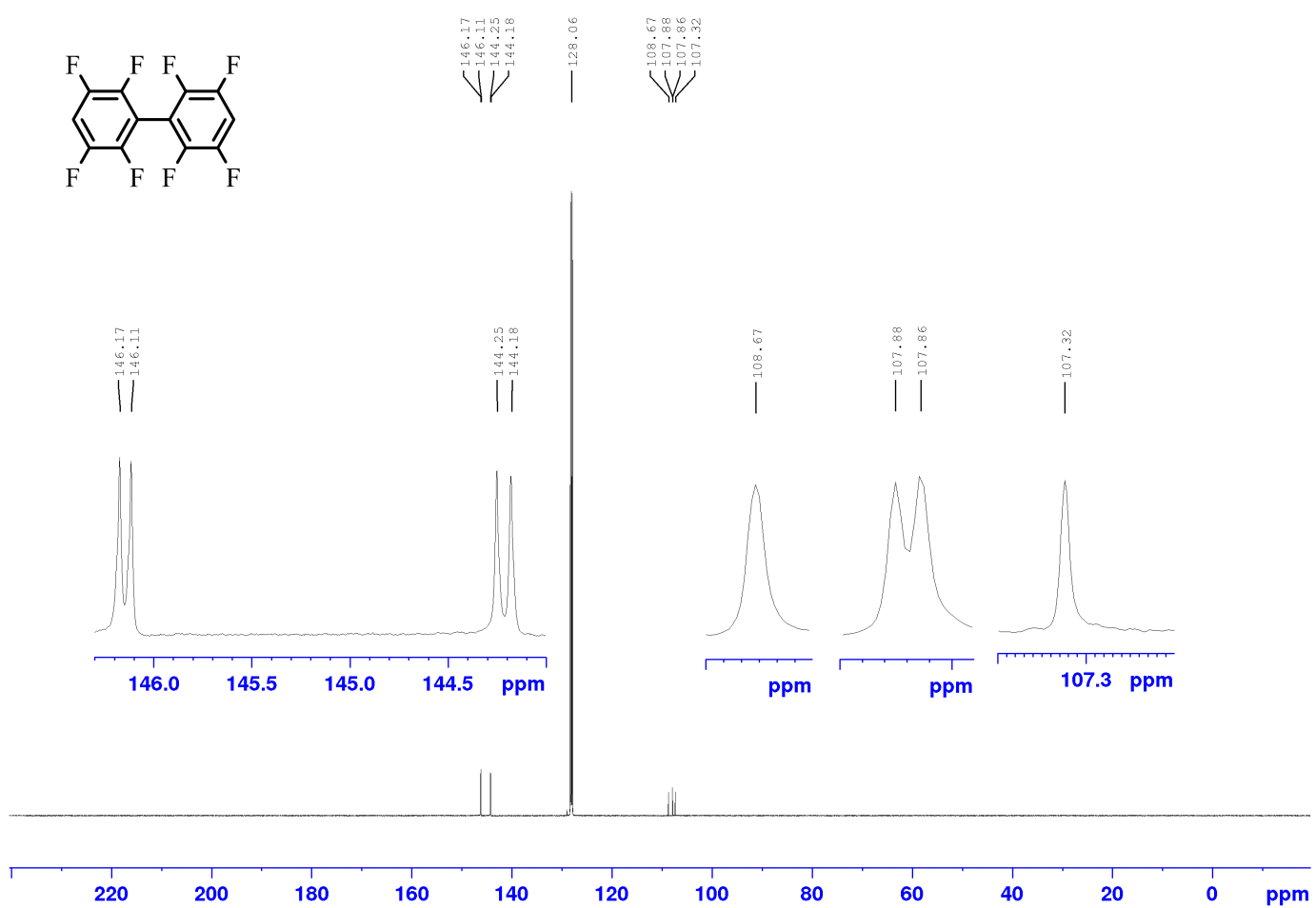

${ }^{19} \mathrm{~F}\left\{{ }^{1} \mathrm{H}\right\}$ NMR spectrum of $2 \mathrm{~d}\left(376 \mathrm{MHz}, \mathrm{C}_{6} \mathrm{D}_{6}\right)$

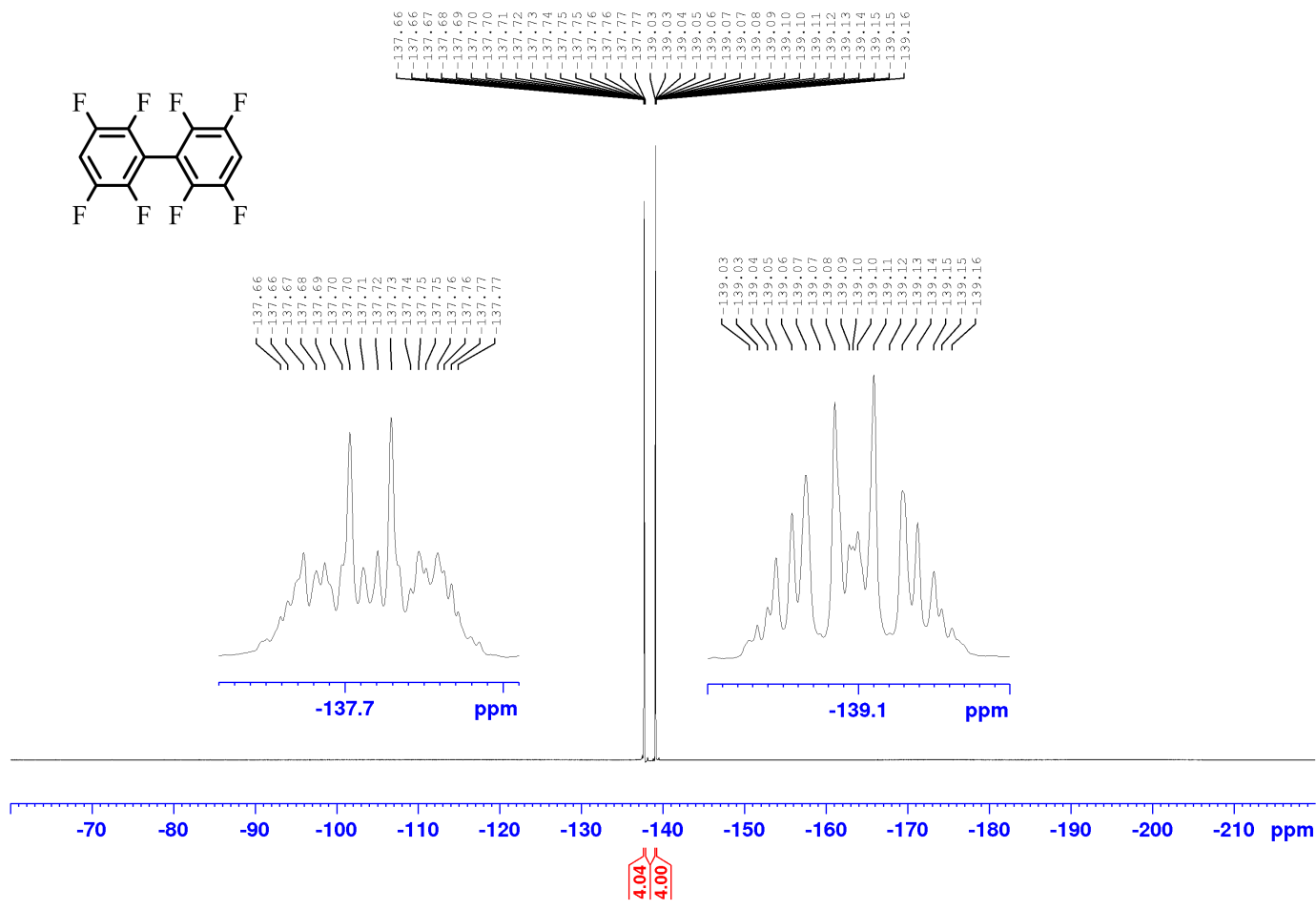




\section{GC-MS of 2d}

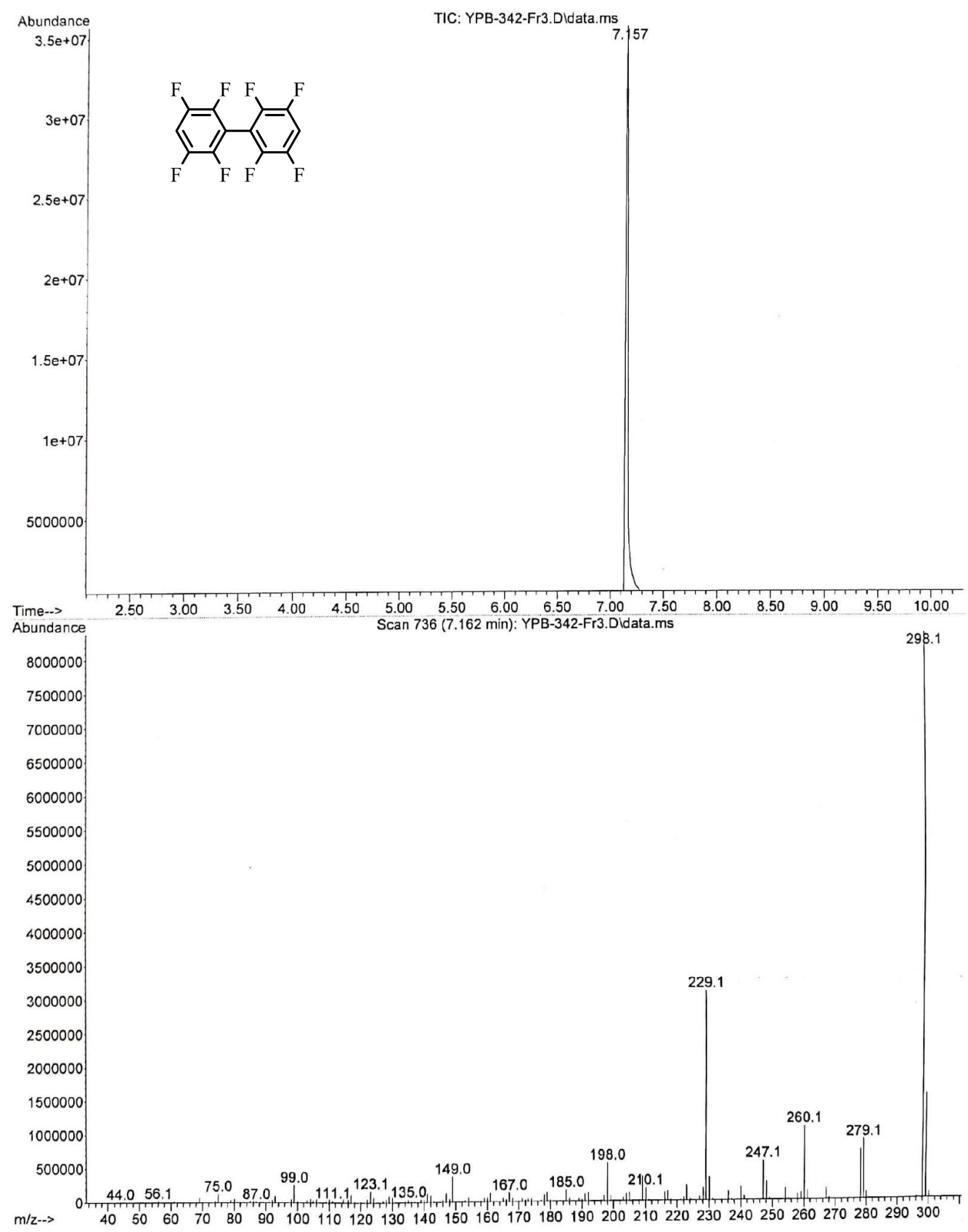


${ }^{1} \mathrm{H}$ NMR spectrum of $2 \mathrm{e}\left(\mathrm{CDCl}_{3}, 500 \mathrm{MHz}\right)$

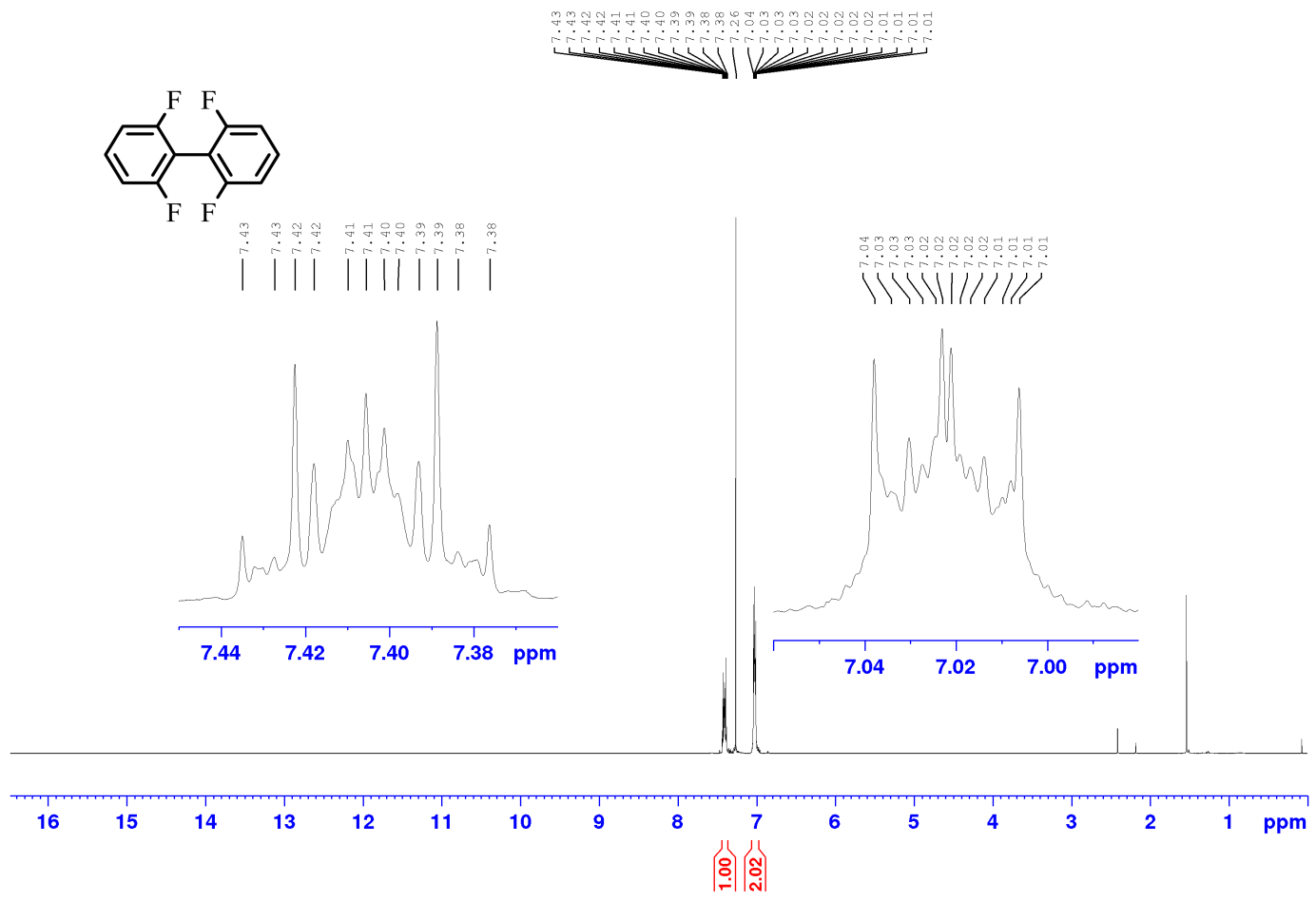

${ }^{13} \mathrm{C}\left\{{ }^{1} \mathrm{H}\right\}$ NMR spectrum of $2 \mathrm{e}\left(125 \mathrm{MHz}, \mathrm{CDCl}_{3}\right)$<smiles>Fc1cccc(F)c1-c1c(F)cccc1F</smiles>
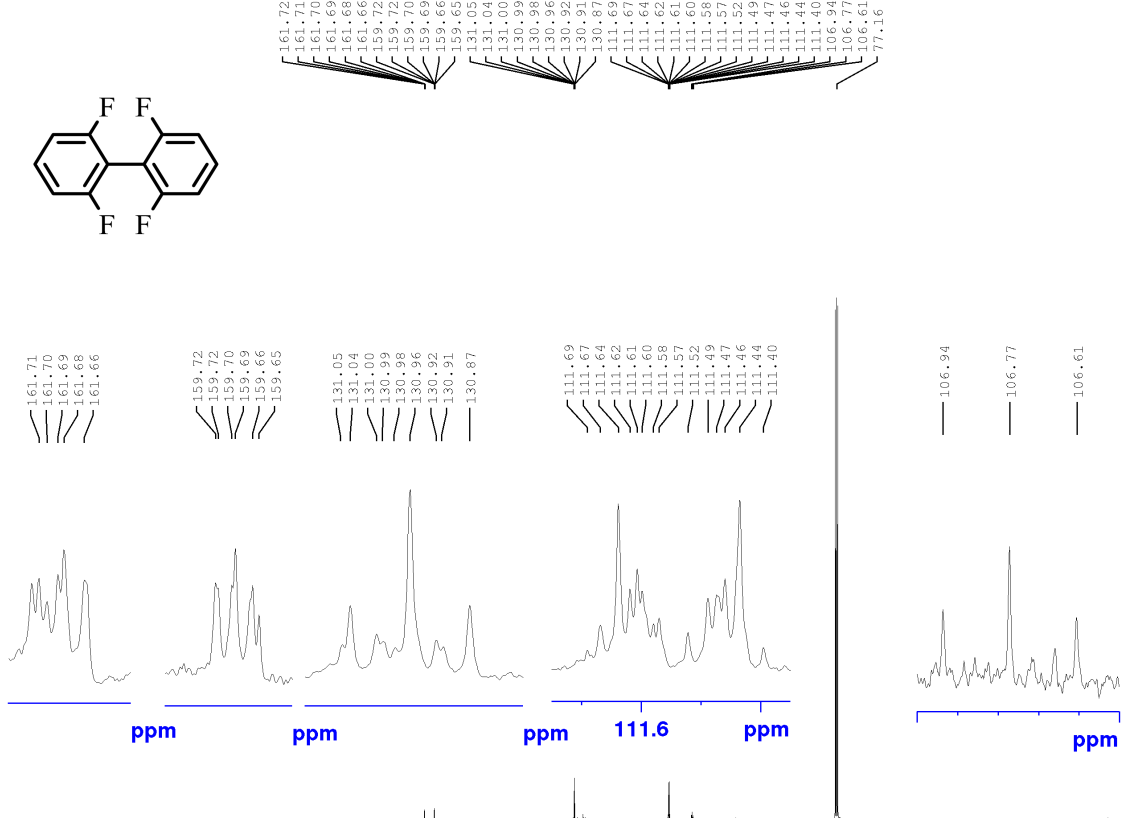

240

220200

160

140

120

$20 \quad 0 \quad \mathrm{ppm}$ 
${ }^{13} \mathrm{C}\left\{{ }^{19} \mathrm{~F}\right\}$ NMR spectrum of $2 \mathrm{e}\left(125 \mathrm{MHz}, \mathrm{CDCl}_{3}\right)$

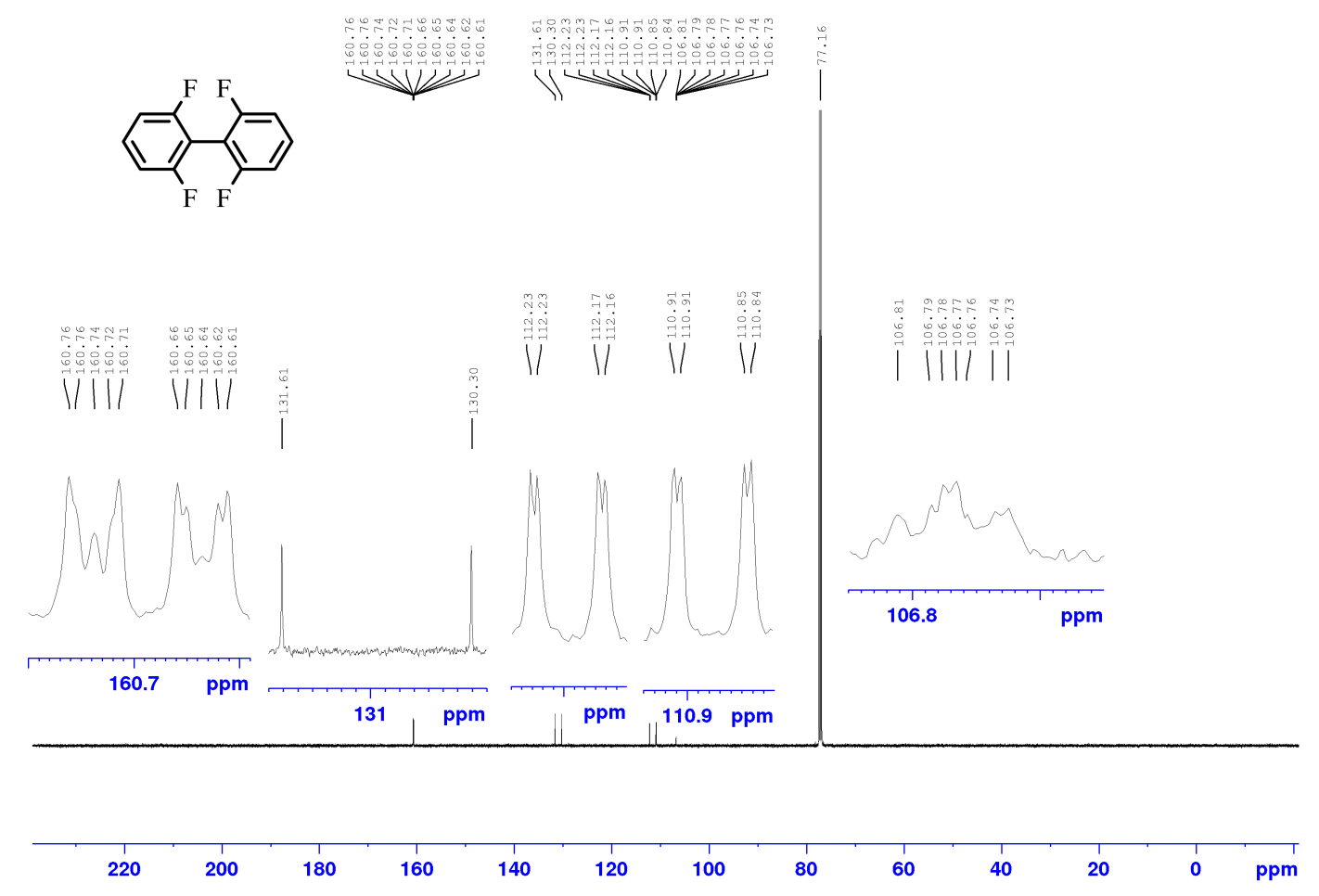

${ }^{19} \mathrm{~F}\left\{{ }^{1} \mathrm{H}\right\}$ NMR spectrum of $2 \mathrm{e}\left(376 \mathrm{MHz}, \mathrm{CDCl}_{3}\right)$<smiles>Fc1cccc(F)c1-c1c(F)cccc1F</smiles>

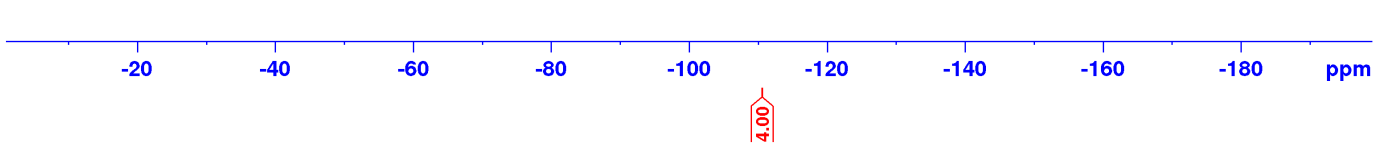




\section{GC-MS of 2e}

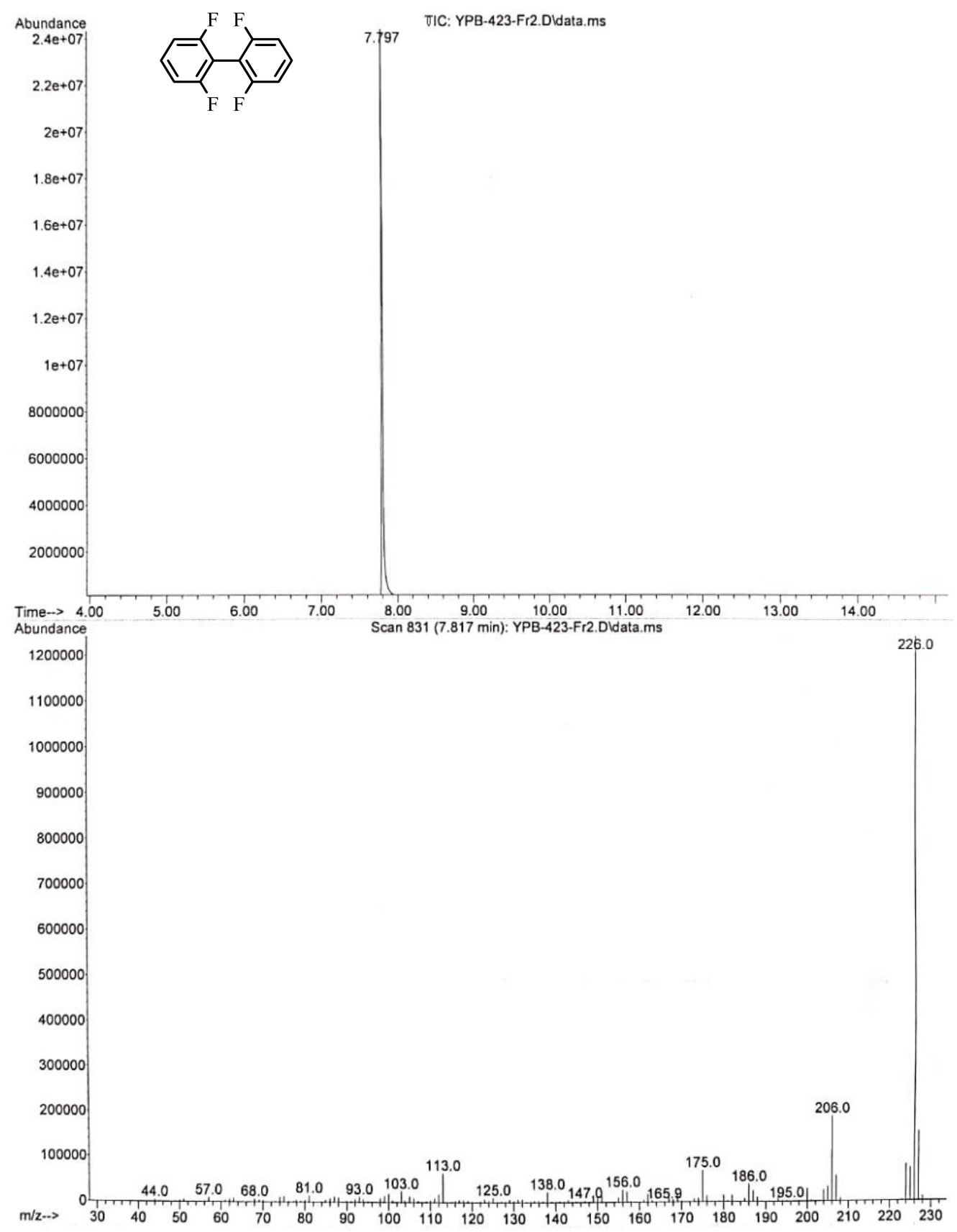


${ }^{1} \mathrm{H}$ NMR spectrum of $2 \mathrm{f}\left(300 \mathrm{MHz}, \mathrm{C}_{6} \mathrm{D}_{6}\right)$
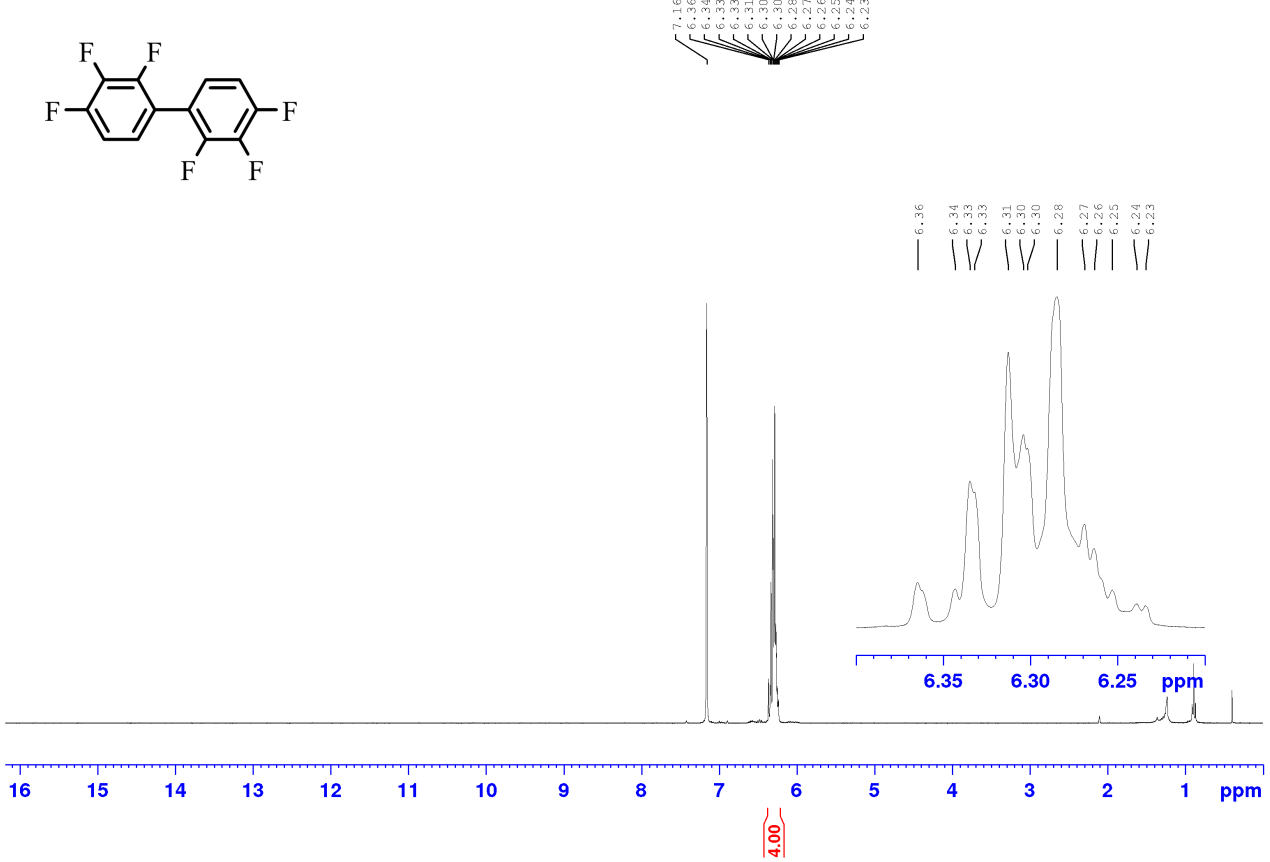

${ }^{13} \mathrm{C}\left\{{ }^{1} \mathrm{H}\right\}$ NMR spectrum of $2 \mathrm{f}\left(125 \mathrm{MHz}, \mathrm{C}_{6} \mathrm{D}_{6}\right)$

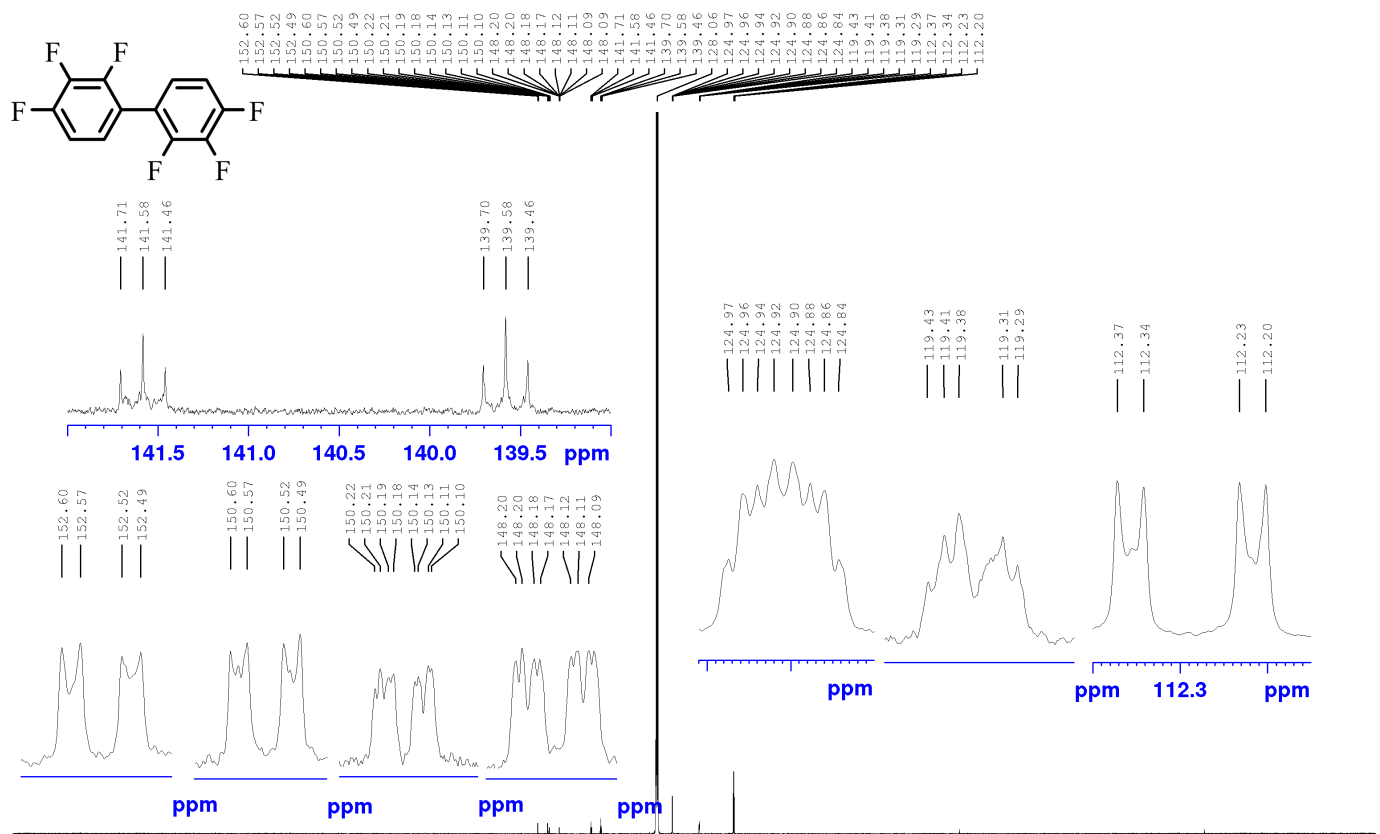

240

220200

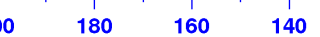

120

$\begin{array}{lllll}60 & 40 & 20 & 0 & \mathrm{ppm}\end{array}$ 
${ }^{13} \mathrm{C}\left\{{ }^{19} \mathrm{~F}\right\}$ NMR spectrum of $2 \mathrm{f}\left(125 \mathrm{MHz}, \mathrm{C}_{6} \mathrm{D}_{6}\right)$

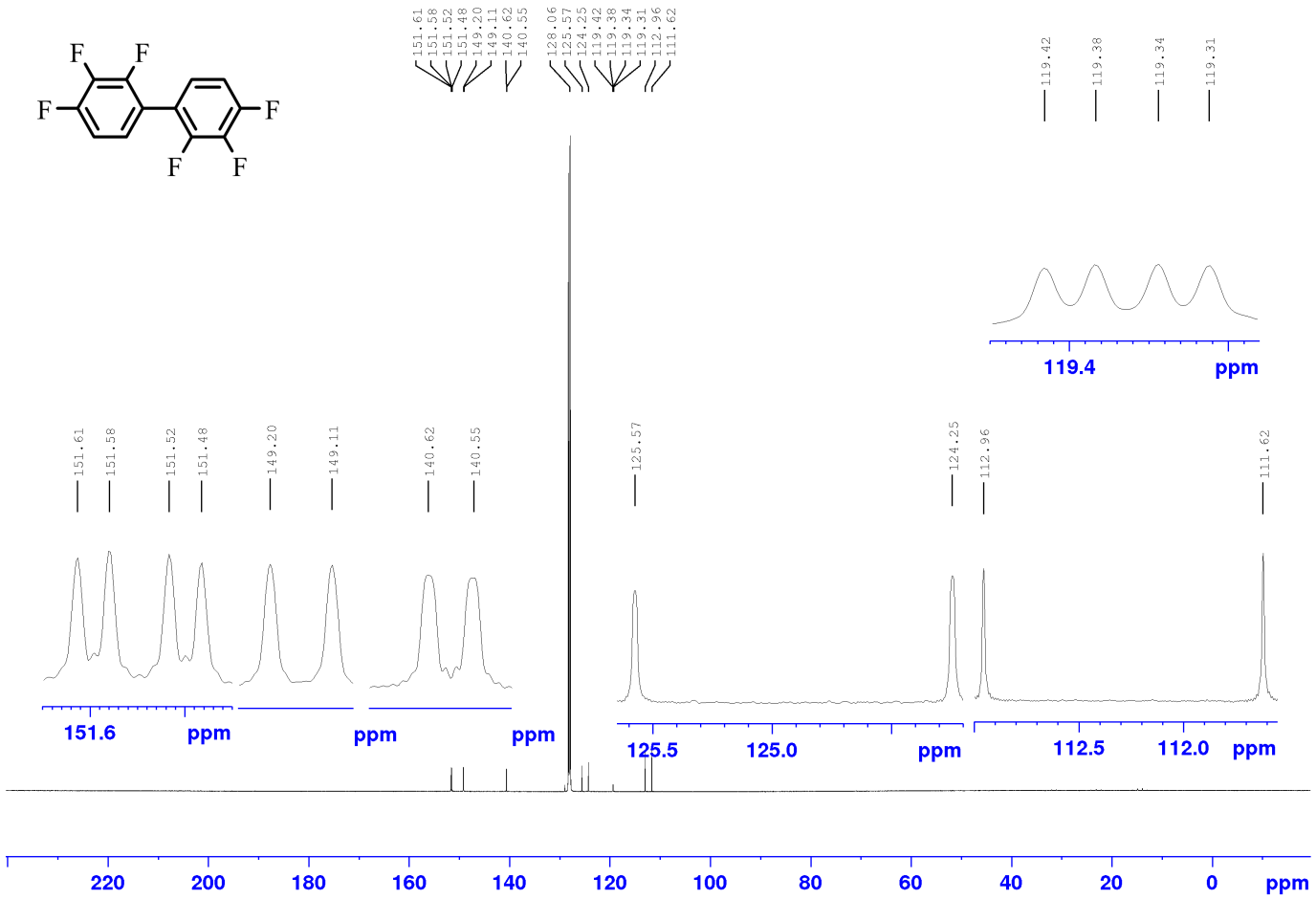

${ }^{19} \mathrm{~F}\left\{{ }^{1} \mathrm{H}\right\}$ NMR spectrum of $2 \mathrm{f}\left(376 \mathrm{MHz}, \mathrm{C}_{6} \mathrm{D}_{6}\right)$
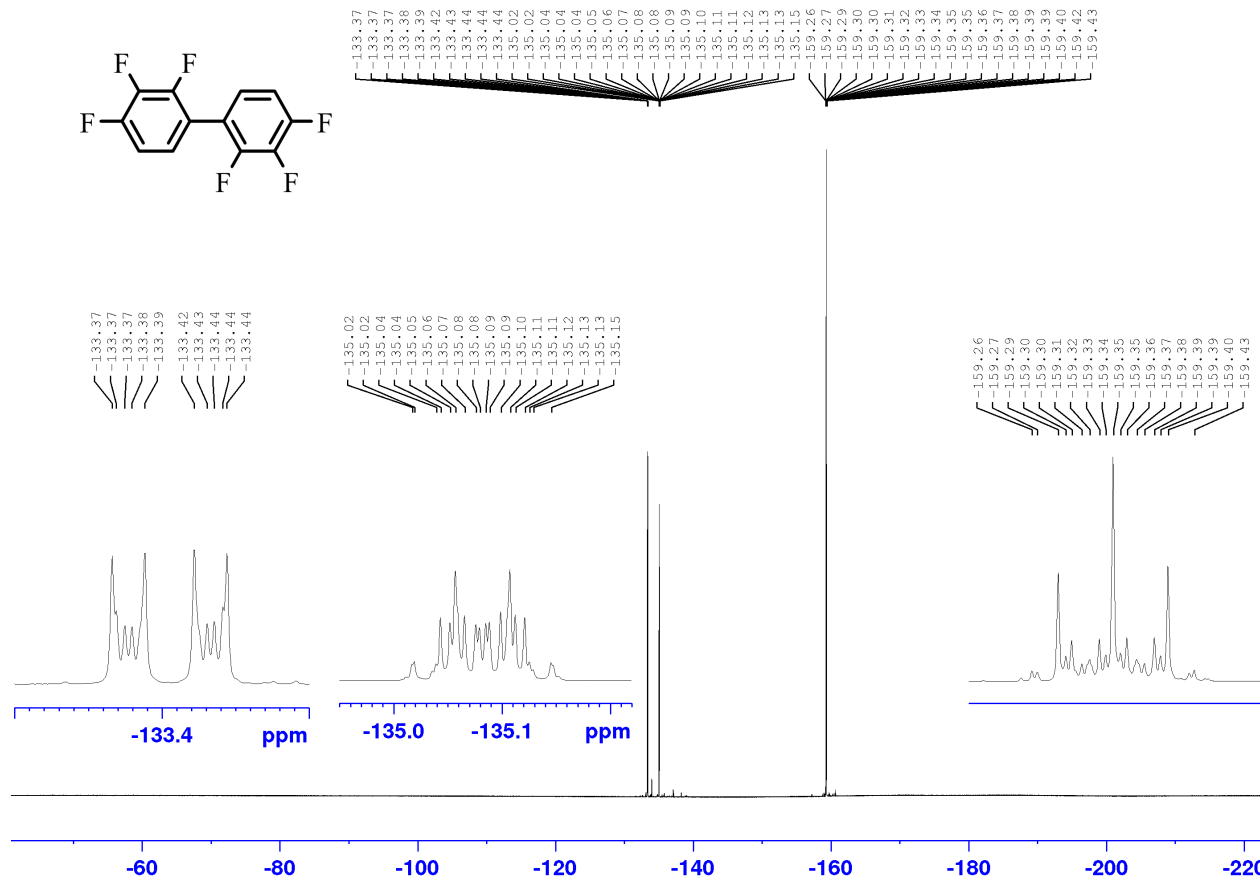

$-80$

$-100$

$-120$

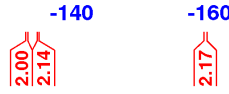




\section{GC-MS of $2 f$}

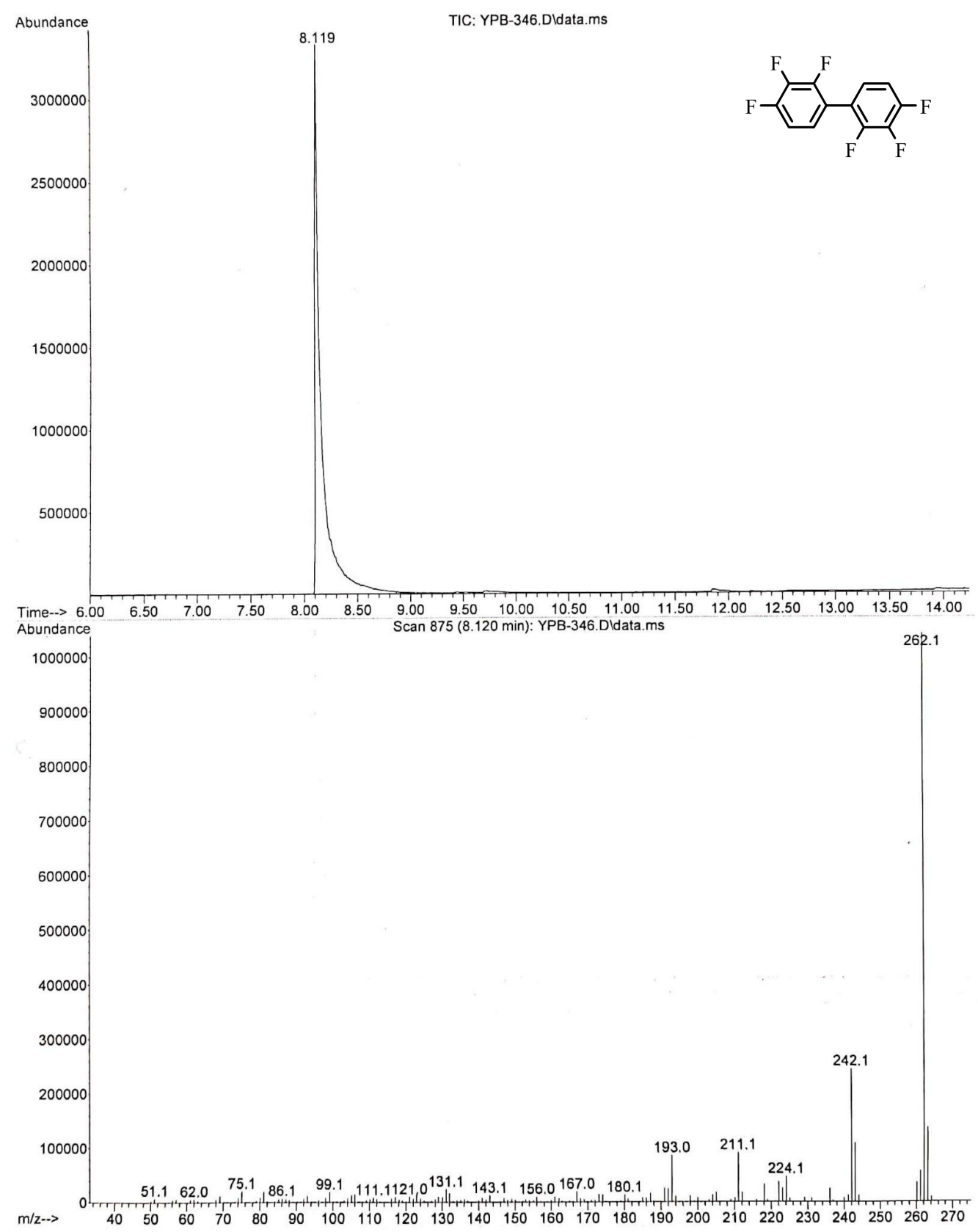


${ }^{1} \mathrm{H}$ NMR spectrum of $2 \mathrm{~g}\left(300 \mathrm{MHz}, \mathrm{CDCl}_{3}\right)$
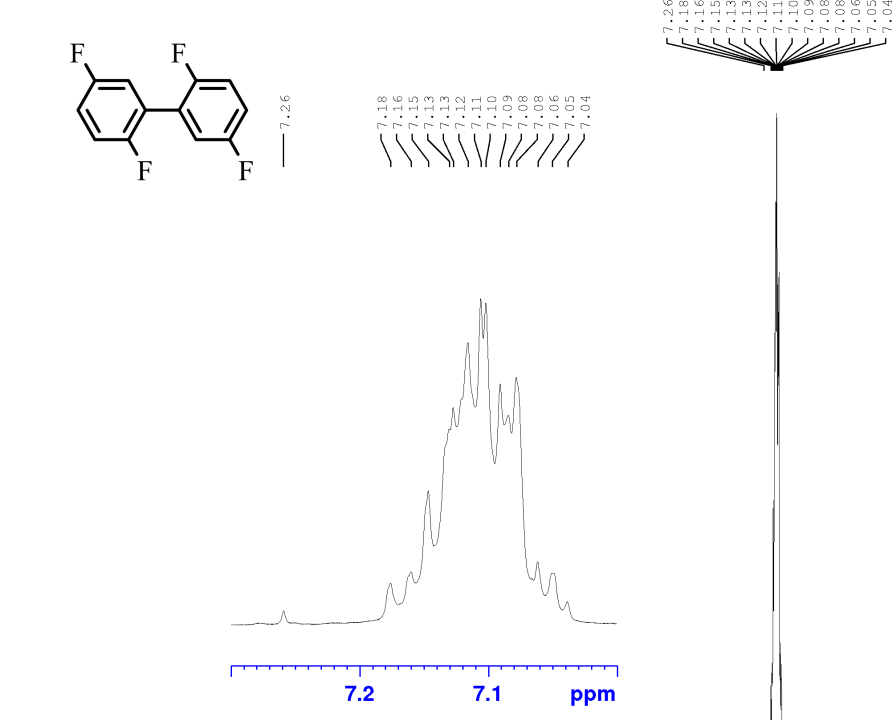

${ }^{13} \mathrm{C}\left\{{ }^{1} \mathrm{H}\right\}$ NMR spectrum of $2 \mathrm{~g}\left(125 \mathrm{MHz}, \mathrm{CDCl}_{3}\right)$

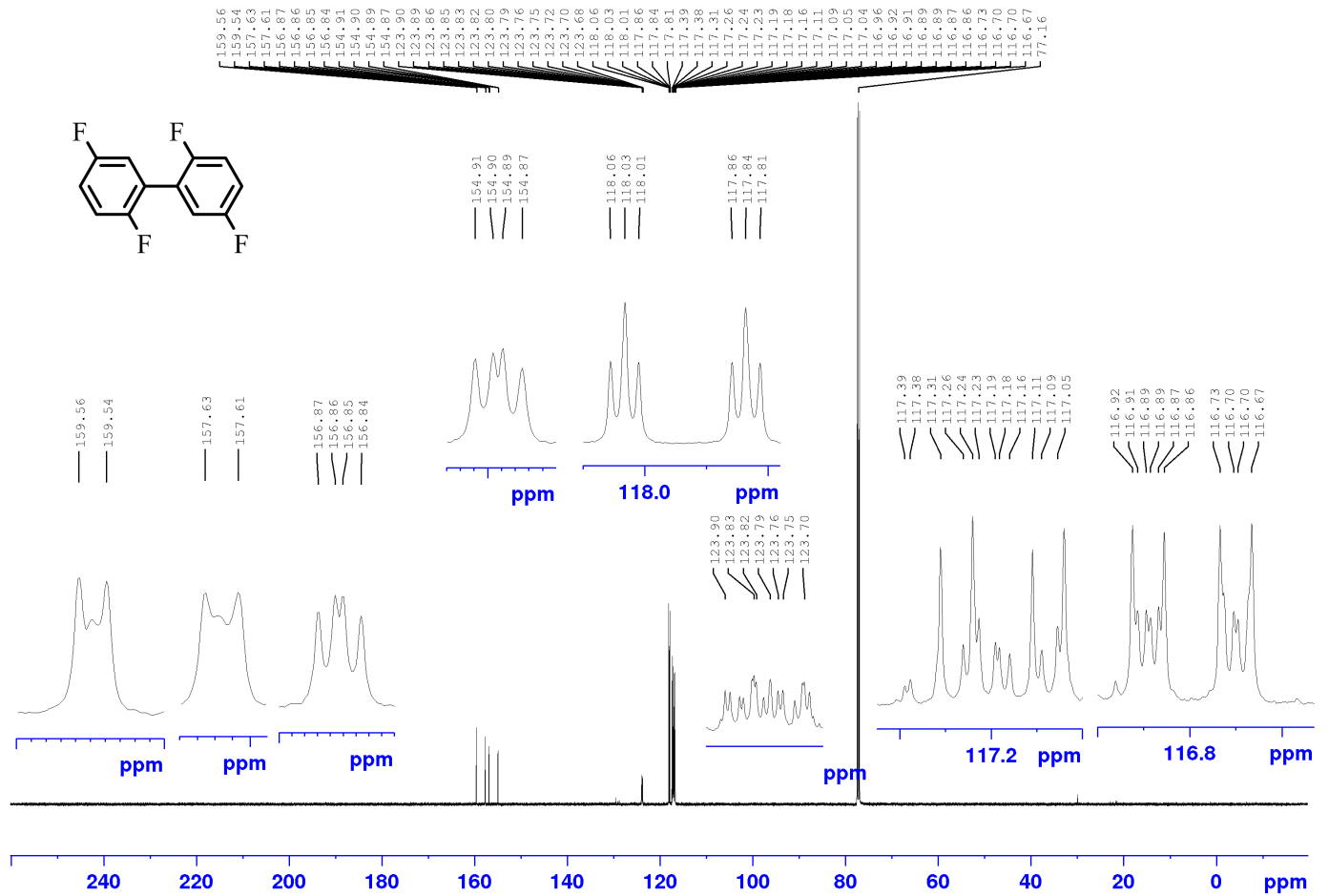


${ }^{19} \mathrm{~F}\left\{{ }^{1} \mathrm{H}\right\}$ NMR spectrum of $2 \mathrm{~g}\left(376 \mathrm{MHz}, \mathrm{CDCl}_{3}\right)$

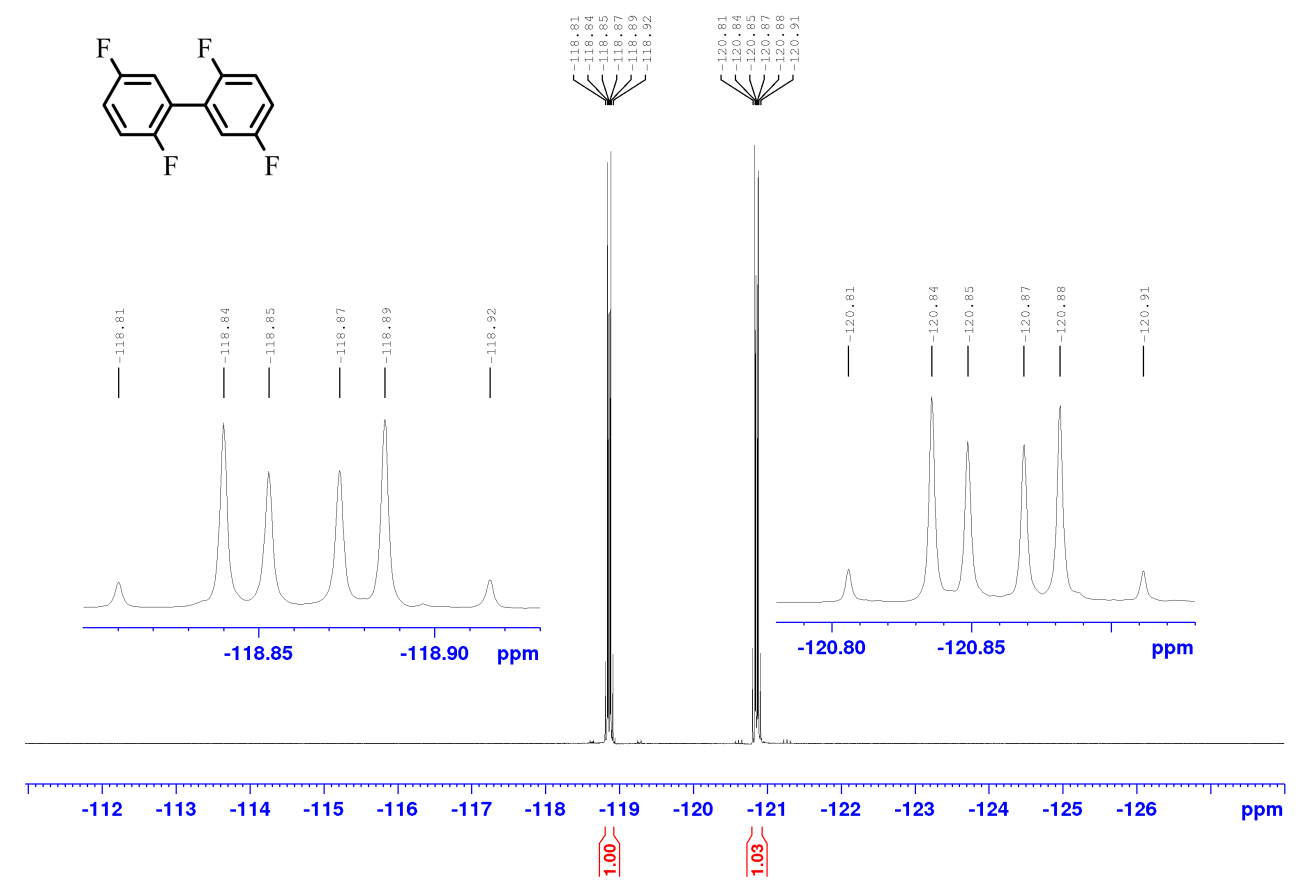

\section{GC-MS of $2 \mathrm{~g}$}

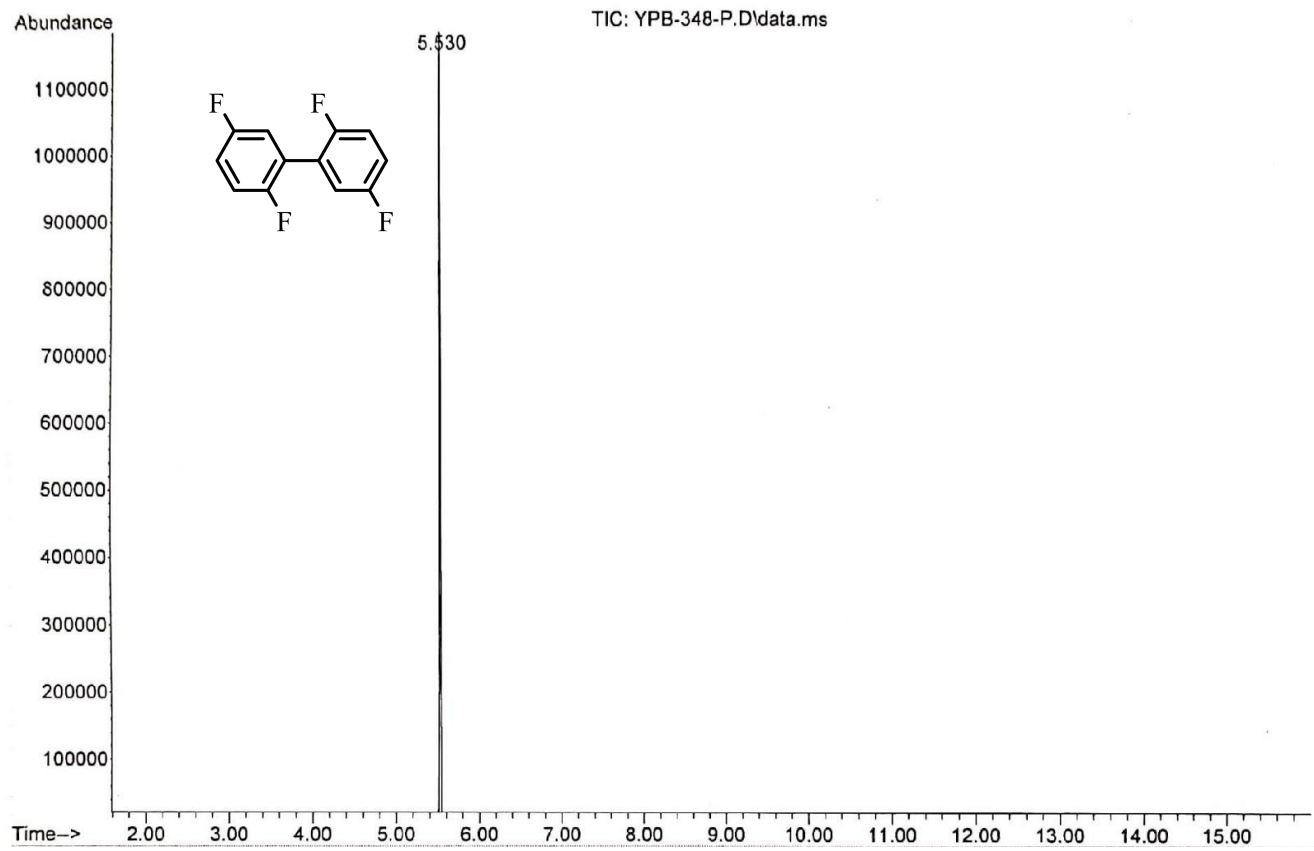




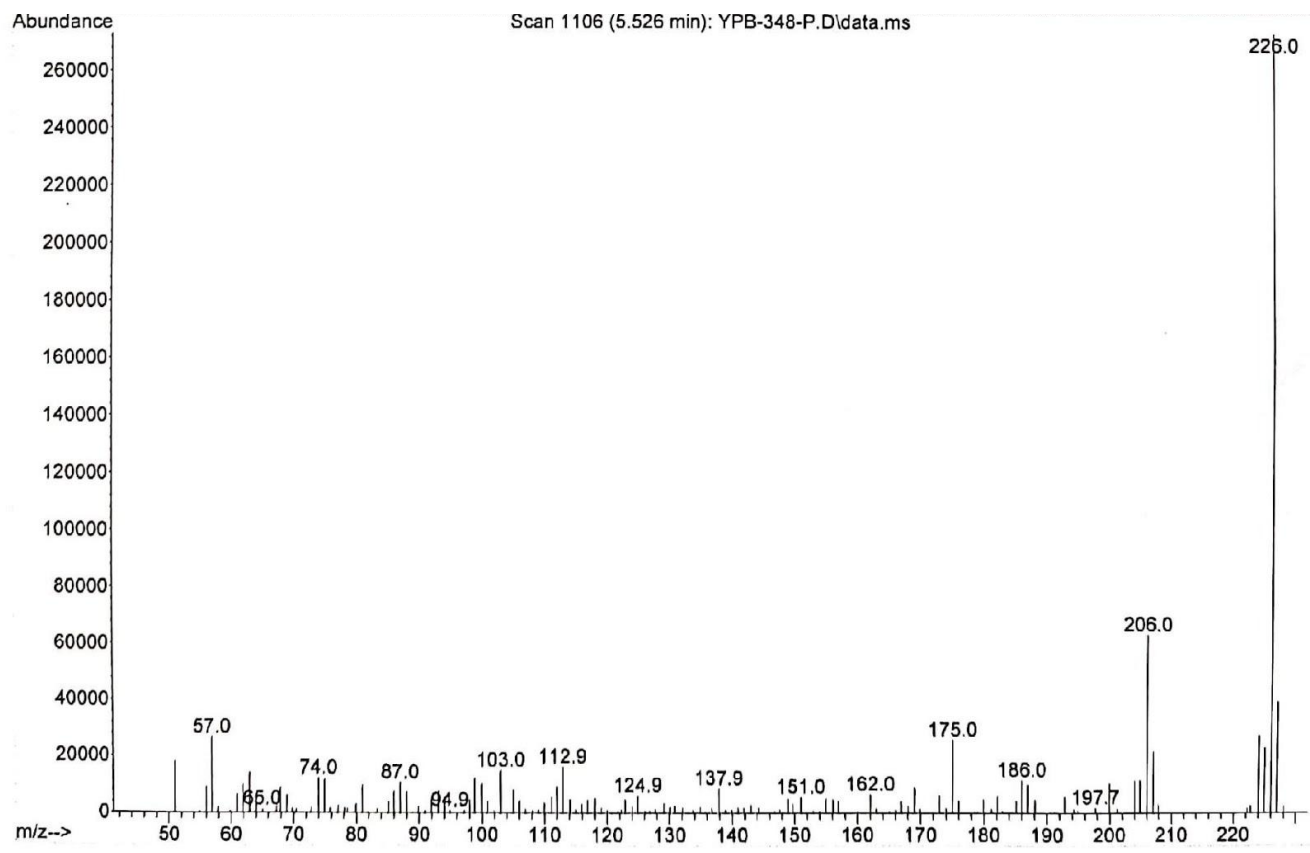

${ }^{1} \mathrm{H}$ NMR spectrum of $2 \mathrm{~h}\left(500 \mathrm{MHz}, \mathrm{CDCl}_{3}\right)$

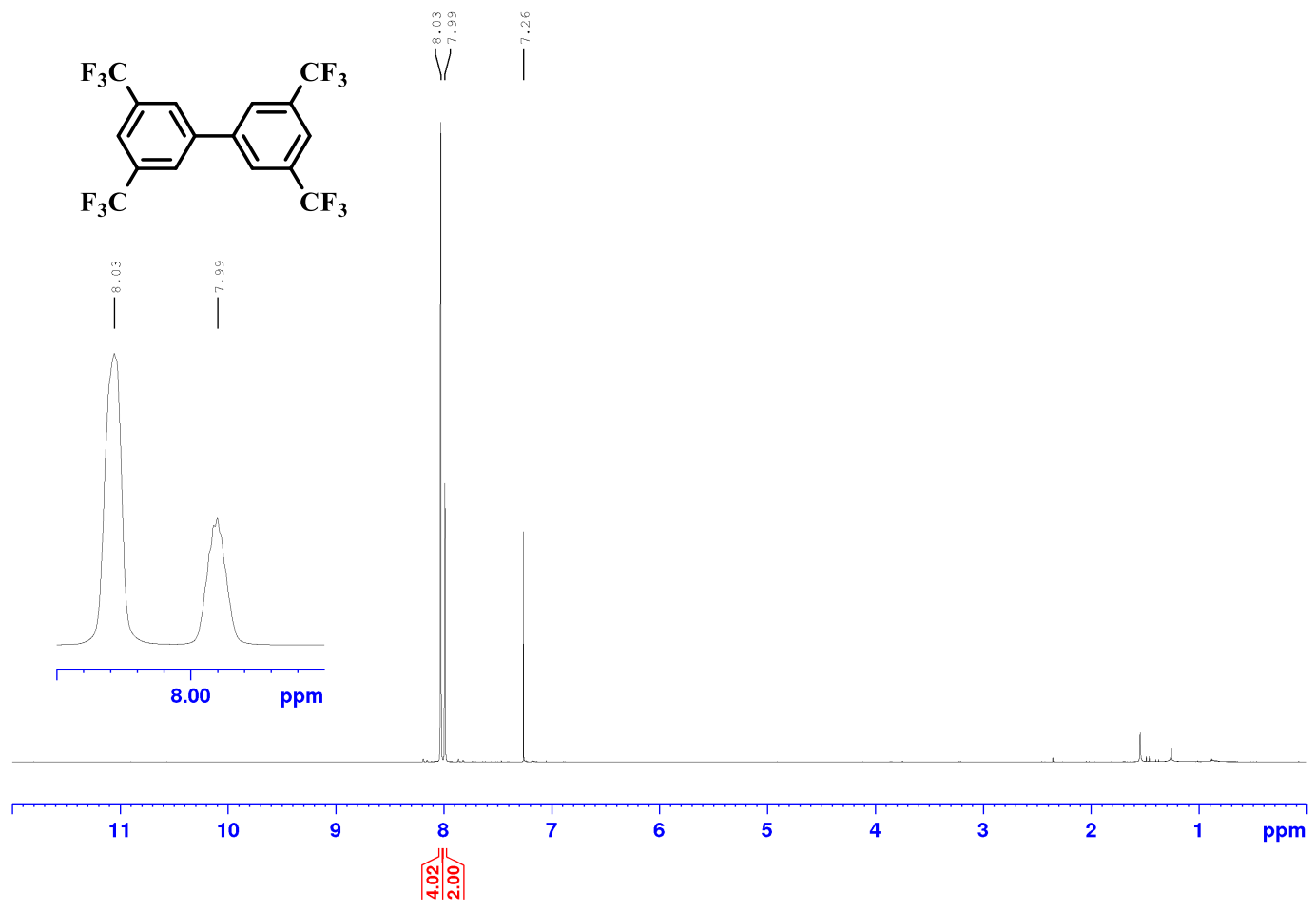


${ }^{13} \mathrm{C}\left\{{ }^{1} \mathrm{H}\right\}$ NMR spectrum of $2 \mathrm{~h}\left(125 \mathrm{MHz}, \mathrm{CDCl}_{3}\right)$
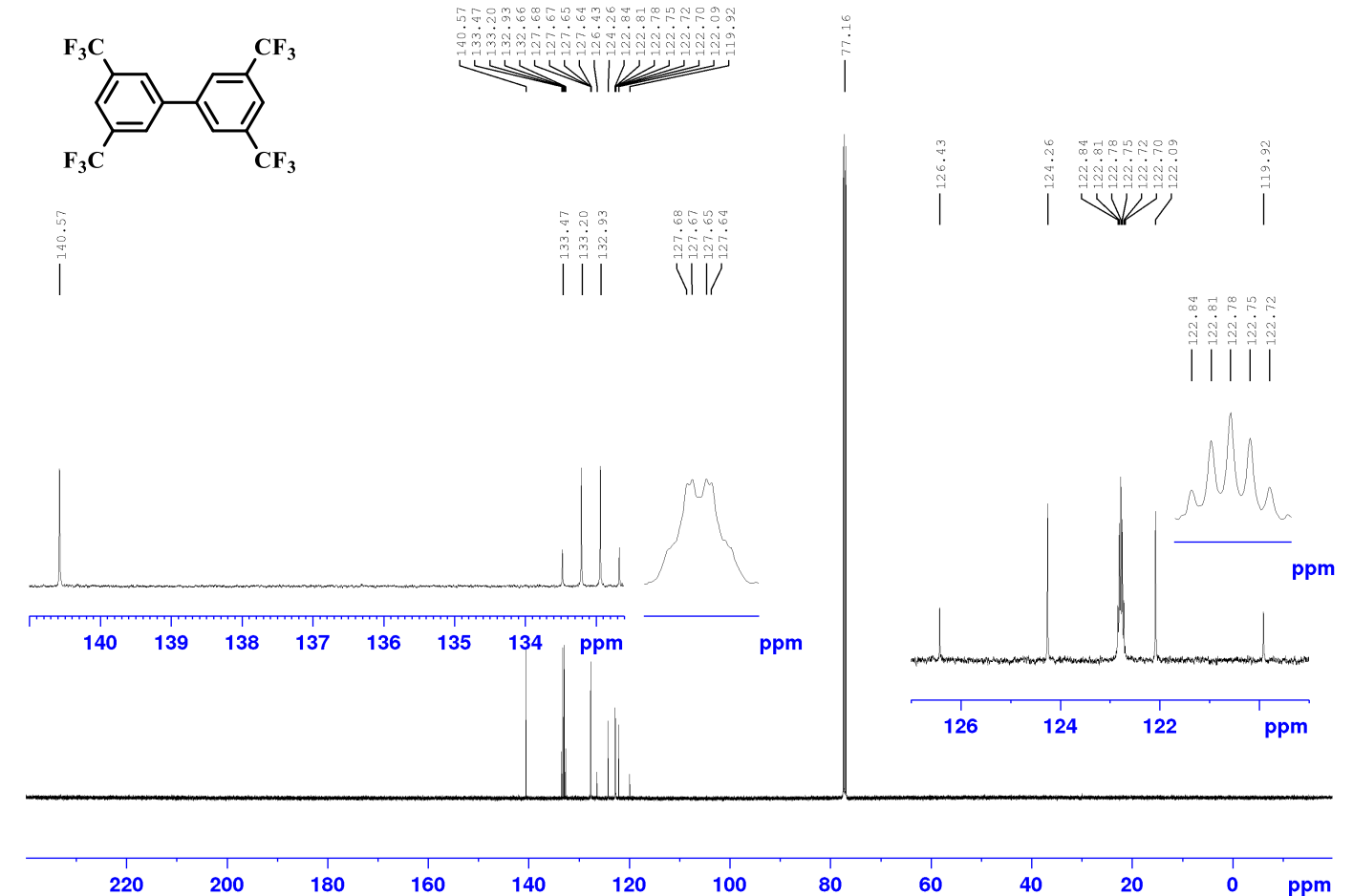

${ }^{19}$ F NMR spectrum of $2 \mathrm{~h}\left(470 \mathrm{MHz}, \mathrm{CDCl}_{3}\right)$<smiles>[CH2+]c1cc(C)cc(-c2cc(C)cc(C(F)(F)F)c2)c1</smiles> 


\section{GC-MS of $2 h$}

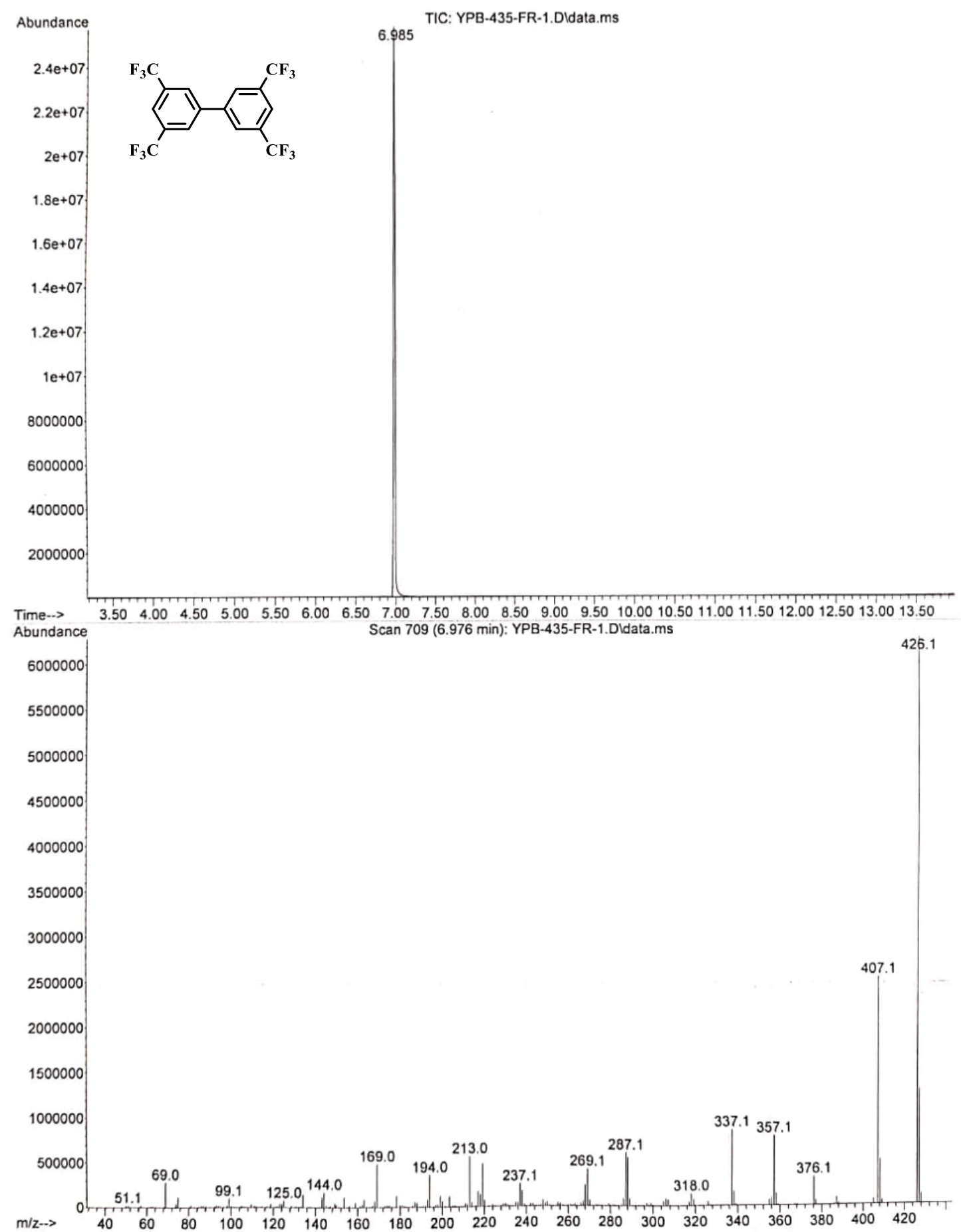




\section{X-ray Crystallography}

Crystal structure determination. Crystals suitable for single-crystal X-ray diffraction were selected, coated in perfluoropolyether oil, and mounted on MiTeGen sample holders. Diffraction data of 2d, 3a, and $\mathbf{3 b}$ were collected on Bruker X8 Apex II 4-circle diffractometers with $\mathrm{CCD}$ area detectors using Mo-K $\alpha$ radiation monochromated by multilayer focusing mirrors. Diffraction data of $\mathbf{2} \mathbf{f}$ and $\mathbf{3 e}$ were collected on a Rigaku Oxford Diffraction XtaLAB Synergy diffractometer with a semiconductor HPA-detector (HyPix6000 ) and multi-layer mirror monochromated $\mathrm{Cu}-\mathrm{K} \alpha$ radiation for the $\mathbf{2 f}$ and $\mathrm{Mo}-\mathrm{K} \alpha$ for the 3e. The crystals were cooled using an Oxford Cryostreams low-temperature device. Data were collected at 100 or $103 \mathrm{~K}$. The images were processed and corrected for Lorentzpolarization effects and absorption as implemented in the Bruker software packages (2d, 3a, 3b) or using the CrysAlis ${ }^{\text {Pro }}$ software from Rigaku Oxford Diffraction (2f, 3e). The structures were solved using the intrinsic phasing method $\left(\right.$ SHELXT) ${ }^{\mathrm{S} 11}$ and Fourier expansion technique. All non-hydrogen atoms were refined in anisotropic approximation, with hydrogen atoms 'riding' in idealized positions, by full-matrix least squares against $\mathrm{F}^{2}$ of all data, using SHELXL ${ }^{\mathrm{S} 12}$ software and the SHELXLE graphical user interface. ${ }^{\mathrm{S} 13}$ Diamond $^{\mathrm{S} 14}$ software was used for graphical representation. Crystal data and experimental details are listed in Table S1; full structural information has been deposited with the Cambridge Crystallographic Data Centre. CCDC-1939238 (2d), 1951276 (2f), 1939239 (3a), 1939240 (3b), $1953484(\mathbf{3 e})$. 
Table S1: Single-crystal X-ray diffraction data and structure refinements of 2d, 2f, 3a, 3b and $3 \mathbf{e}$.

\begin{tabular}{|c|c|c|c|c|c|}
\hline Data & $2 \mathbf{d}^{[\mathrm{a}]}$ & 2f & $\mathbf{3 a}^{[\mathrm{a}]}$ & $\mathbf{3 b}^{[\mathrm{a}]}$ & $3 e$ \\
\hline CCDC number & 1939238 & 1951276 & 1939239 & 1939240 & 1953484 \\
\hline Empirical formula & $\mathrm{C}_{12} \mathrm{H}_{2} \mathrm{~F}_{8}$ & $\mathrm{C}_{12} \mathrm{H}_{4} \mathrm{~F}_{6}$ & $\begin{array}{l}\mathrm{C}_{16} \mathrm{H}_{6} \mathrm{~F}_{10} \mathrm{~N}_{2} \mathrm{Pd} \\
\cdot 0.5\left(\mathrm{C}_{4} \mathrm{H}_{10} \mathrm{O}\right) \\
\end{array}$ & $\mathrm{C}_{16} \mathrm{H}_{10} \mathrm{~F}_{6} \mathrm{~N}_{2} \mathrm{Pd}$ & $\mathrm{C}_{16} \mathrm{H}_{12} \mathrm{~F}_{4} \mathrm{~N}_{2} \mathrm{Pd}$ \\
\hline $\begin{array}{c}\text { Formula weight / } \\
\mathrm{g} \cdot \mathrm{mol}^{-1}\end{array}$ & 298.14 & 262.15 & 559.69 & 450.66 & 414.68 \\
\hline$T / \mathrm{K}$ & $103(2)$ & $100(2)$ & $103(2)$ & $100(2)$ & $100(2)$ \\
\hline Radiation, $\lambda / \AA$ & $\operatorname{MoK} \alpha 0.71073$ & $\mathrm{CuK} \alpha 1.54184$ & MoK $\alpha 0.71073$ & MoK $\alpha 0.71073$ & MoK $\alpha 0.71073$ \\
\hline Crystal size $/ \mathrm{mm}^{3}$ & $\begin{array}{c}0.20 \times 0.25 \times \\
0.26 \\
\end{array}$ & $\begin{array}{c}0.76 \times 0.08 \times \\
0.04\end{array}$ & $\begin{array}{c}0.17 \times 0.18 \times \\
0.32\end{array}$ & $\begin{array}{c}0.09 \times 0.19 \times \\
0.28\end{array}$ & $\begin{array}{c}0.15 \times 0.11 \times \\
0.09\end{array}$ \\
\hline $\begin{array}{c}\text { Crystal color, } \\
\text { habit }\end{array}$ & colorless block & $\begin{array}{c}\text { colorless } \\
\text { needle }\end{array}$ & colorless block & colorless block & colorless plate \\
\hline$\mu / \mathrm{mm}^{-1}$ & 0.215 & 1.677 & 1.080 & 1.208 & 1.211 \\
\hline Crystal system & orthorhombic & orthorhombic & orthorhombic & monoclinic & monoclinic \\
\hline Space group & $P b c a$ & $F d d 2$ & Pnna & $P 2_{1} / n$ & $P 2_{1}$ \\
\hline$a / \AA$ & 10.994(3) & $40.0791(8)$ & $8.612(3)$ & $9.5500(15)$ & $9.1923(3)$ \\
\hline$b / \AA$ & $8.897(4)$ & $13.0995(3)$ & $14.566(6)$ & $17.778(5)$ & $8.9775(2)$ \\
\hline$c / \AA$ & $20.967(3)$ & $3.69800(10)$ & $15.189(5)$ & $19.108(3)$ & $9.5988(3)$ \\
\hline$\alpha /^{\circ}$ & 90 & 90 & 90 & 90 & 90 \\
\hline$\beta /{ }^{\circ}$ & 90 & 90 & 90 & $90.415(7)$ & $90.225(3)$ \\
\hline$\gamma /{ }^{\circ}$ & 90 & 90 & 90 & 90 & 90 \\
\hline Volume / $\AA^{3}$ & $2050.9(11)$ & $1941.51(8)$ & $1905.4(13)$ & $3244.2(12)$ & $792.13(4)$ \\
\hline$Z$ & 8 & 8 & 4 & 8 & 2 \\
\hline$\rho_{\text {calc }} / \mathrm{g} \cdot \mathrm{cm}^{-3}$ & 1.931 & 1.794 & 1.951 & 1.845 & 1.739 \\
\hline$F(000)$ & 1168 & 1040 & 1092 & 1760 & 408 \\
\hline$\theta$ range $/{ }^{\circ}$ & $1.943-28.376$ & $4.413-74.263$ & $1.937-28.360$ & $1.565-29.130$ & $4.244-61.014$ \\
\hline $\begin{array}{c}\text { Reflections } \\
\text { collected }\end{array}$ & 37562 & 8216 & 18707 & 69798 & 24242 \\
\hline Unique reflections & 2565 & 850 & 2386 & 8733 & 4817 \\
\hline $\begin{array}{c}\text { Parameters / } \\
\text { restraints }\end{array}$ & $181 / 0$ & $82 / 1$ & $180 / 82$ & $455 / 0$ & $210 / 0$ \\
\hline GooF on $F^{2}$ & 1.041 & 1.056 & 1.074 & 1.063 & 1.044 \\
\hline $\mathrm{R}_{1}[\mathrm{I}>2 \sigma(\mathrm{I})]$ & 0.0319 & 0.0317 & 0.0310 & 0.0294 & 0.0266 \\
\hline$w \mathrm{R}^{2}$ (all data) & 0.0900 & 0.0813 & 0.0794 & 0.0770 & 0.0588 \\
\hline $\begin{array}{c}\text { Max. / min. } \\
\text { residual electron } \\
\text { density / e } \cdot \AA^{-3}\end{array}$ & $0.498 /-0.236$ & $0.193 /-0.221$ & $0.907 /-0.494$ & $0.706 /-0.868$ & $1.06 /-0.53$ \\
\hline
\end{tabular}

${ }^{[a]}$ For compounds $\mathbf{2 d}, \mathbf{3 a}$, and $\mathbf{3 b}$, the unit-cell parameters and their standard deviations were obtained from a combination of the random-error standard deviations from the original least-squares refinement with the systematic-error component estimated from 32 Monte-Carlo simulations using the Bruker software package. For this reason, the standard deviations of the unit-cell parameters are significantly larger for these compounds than for $\mathbf{2} \mathbf{f}$ and $\mathbf{3 e}$, and they represent a better overall estimation of the realistic standard deviations.

Table S2. Intermolecular $\mathrm{C}-\mathrm{H} \cdots \mathrm{F}-\mathrm{C}, \mathrm{H} \cdots \mathrm{F}, \mathrm{C} \cdots \mathrm{F}$, and $\mathrm{F} \cdots \mathrm{F}$ interaction distances $(\AA)$ and angles $\left(^{\circ}\right)$ in compounds $\mathbf{2 d}$ and $\mathbf{2 f}$ at $100 \mathrm{~K}$.

\begin{tabular}{|c|c|c|c|c|}
\hline Compound & $C-H \cdots F / F \cdots F$ & $\mathbf{H} \cdots \mathrm{F}$ & $\mathbf{C} / \mathbf{F} \cdots \mathrm{F}$ & $\angle$ (CHF) \\
\hline \multirow{4}{*}{ 2d } & $\mathrm{C} 10-\mathrm{H} 10 \cdots \mathrm{F} 1$ & $2.3949(9)$ & $3.0376(15)$ & $124.62(8)$ \\
\hline & F2 $\cdots$ F5 & & $2.7078(12)$ & \\
\hline & F3 $\cdots F 8$ & & $2.8717(12)$ & \\
\hline & F6 $\cdots$ F7 & & $2.9169(15)$ & \\
\hline \multirow{2}{*}{$2 f$} & $\mathrm{C} 4-\mathrm{H} 4 \cdots \mathrm{F} 2$ & $2.6021(13)$ & $3.261(3)$ & $126.77(14)$ \\
\hline & $\mathrm{F} 1 \cdots \mathrm{F} 1$ & & $2.923(2)$ & \\
\hline
\end{tabular}




\section{Computational Details}

All computations were performed using the Gaussian 09 (Revision E.01) package. ${ }^{\text {15 }}$ Geometry optimizations, without any symmetry constraints, and frequency calculations were performed using the hybrid DFT functional B3LYP ${ }^{S 16}$ augmented with Tomasi's polarizable continuum model (PCM), ${ }^{\mathrm{S} 17}$ Grimme's dispersion correction (GD3) and Becke-Johnson damping (BJ). ${ }^{\mathrm{S} 18}$ For these calculations, the SDD basis set for palladium was used and the 6$31 \mathrm{G}^{* *}$ basis set for all other atoms was used. Transition state geometries were obtained using opt $=($ ts, noeigentest, calcfc $)$ algorithms. ${ }^{\mathrm{S} 19}$ All optimized transition state structures were verified as maxima with only one imaginary frequency, and the magnitudes of all frequencies were greater than the residual frequencies due to rotations and translations. In addition, each transition state established was ensured to be on the preferred reaction path by performing "plus-and-minus-displacement" minimization calculations in which the transition state structure was displaced ca. $0.05 \AA$ or $5^{\circ}$ along the imaginary frequency normal mode in both directions. $^{\text {S20 }}$ The displaced geometries were subsequently optimized to the nearest minimum. Zero-point vibrational energies, and thermal corrections were computed from frequency calculations with a standard state of $298 \mathrm{~K}$ and 1 atm. Single point energy calculations were performed at the same level of theory with the triple-zeta basis sets Def2TZVP (Pd) and 6-311+g(2d,p) (other atoms).

\section{Optimized Structures, Cartesian Coordinates, and Energies}

Note: Coordinates for all computed structures are also included in attached xyz file.

1) $\left[\mathrm{Pd}(\mathrm{MeCN})_{2}\left(\mathrm{C}_{6} \mathrm{~F}_{5}\right)_{2}\right](\mathbf{3 a})$ 


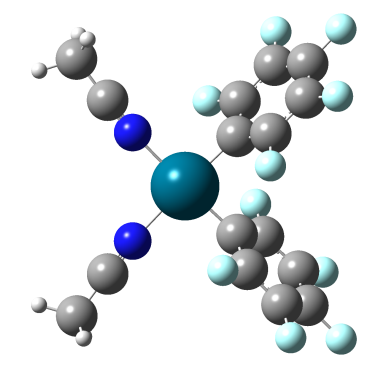

Number of imaginary frequencies $=0$

\section{$\mathrm{E}_{\text {total }}=-1849.14566484 \mathrm{a} . \mathrm{u}$}

$\mathrm{G}_{\text {correction }}=0.127629 \mathrm{a} . \mathrm{u}$

\section{Cartesian coordinates:}

\begin{tabular}{|c|c|c|c|}
\hline $\mathrm{Pd}$ & 0.00000000 & 0.00000000 & 1.32741100 \\
\hline $\mathrm{F}$ & -2.19119000 & 0.81516600 & -0.87967600 \\
\hline $\mathrm{N}$ & 0.03252500 & -1.50791000 & 2.79180400 \\
\hline $\mathrm{C}$ & 0.00000000 & 1.37137200 & -0.13507300 \\
\hline C & -1.07063100 & 1.56851100 & -0.99658300 \\
\hline $\mathrm{F}$ & -2.11945800 & 2.68987700 & -2.81927700 \\
\hline C & 0.06552700 & 3.32714200 & -2.17497700 \\
\hline $\mathrm{F}$ & 2.24848900 & 3.93062200 & -1.4908610 \\
\hline C & -1.05979700 & 2.52655300 & -2.0076970 \\
\hline $\mathrm{F}$ & 0.09504100 & 4.25838200 & -3.1413610 \\
\hline C & 1.15884300 & 3.15902600 & -1.3305650 \\
\hline $\mathrm{F}$ & 2.19672000 & 2.06619000 & 0.4620390 \\
\hline $\mathrm{C}$ & 1.10451300 & 2.19045800 & -0.3322150 \\
\hline C & 0.05059700 & -2.37578700 & 3.5540630 \\
\hline C & 0.07038300 & -3.47280700 & 4.5074770 \\
\hline $\mathrm{H}$ & 0.07311300 & -3.07720200 & 5.5259690 \\
\hline $\mathrm{H}$ & 0.96710700 & -4.07768100 & 4.3520510 \\
\hline $\mathrm{H}$ & -0.81503300 & -4.09714800 & 4.3645610 \\
\hline $\mathrm{F}$ & 2.19119000 & -0.81516600 & -0.8796760 \\
\hline $\mathrm{N}$ & -0.03252500 & 1.50791000 & 2.7918040 \\
\hline C & 0.00000000 & -1.37137200 & -0.1350730 \\
\hline C & 1.07063100 & -1.56851100 & -0.9965830 \\
\hline $\mathrm{F}$ & 2.11945800 & -2.68987700 & -2.8192770 \\
\hline C & -0.06552700 & -3.32714200 & -2.17497700 \\
\hline $\mathrm{F}$ & -2.24848900 & -3.93062200 & -1.4908610 \\
\hline C & 1.05979700 & -2.52655300 & -2.0076970 \\
\hline $\mathrm{F}$ & -0.09504100 & -4.25838200 & -3.1413610 \\
\hline C & -1.15884300 & -3.15902600 & -1.3305650 \\
\hline $\mathrm{F}$ & -2.19672000 & -2.06619000 & 0.462039 \\
\hline C & -1.10451300 & -2.19045800 & -0.3322150 \\
\hline C & -0.05059700 & 2.37578700 & 3.5540630 \\
\hline C & -0.07038300 & 3.47280700 & 4.5074770 \\
\hline $\mathrm{H}$ & -0.07311300 & 3.07720200 & 5.5259690 \\
\hline $\mathrm{H}$ & -0.96710700 & 4.07768100 & 4.3520510 \\
\hline $\mathrm{H}$ & 0.81503300 & 4.09714800 & 4.3645610 \\
\hline
\end{tabular}

\section{2) $\mathrm{TS}(3 \mathrm{a}-4)$}




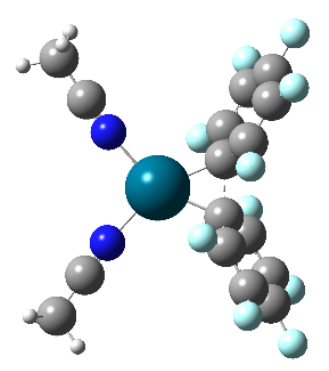

Number of imaginary frequencies $=1\left(i 94.30 \mathrm{~cm}^{-1}\right)$

$\mathrm{E}_{\text {total }}=-1849.09234227 \mathrm{a} . \mathrm{u}$

$\mathrm{G}_{\text {correction }}=0.123003 \mathrm{a} . \mathrm{u}$

\section{Cartesian coordinates:}

\begin{tabular}{|c|c|c|c|}
\hline $\mathrm{Pd}$ & -0.00001200 & 1.39323900 & 0.00020900 \\
\hline $\mathrm{C}$ & -0.83692600 & -0.55872700 & -0.05326400 \\
\hline $\mathrm{C}$ & -1.36419400 & -1.23633400 & -1.18117700 \\
\hline $\mathrm{C}$ & -1.69333400 & -0.61314200 & 1.08386400 \\
\hline C & -2.57999400 & -1.90377100 & -1.18551000 \\
\hline C & -2.91338100 & -1.26722300 & 1.09490200 \\
\hline C & -3.36597200 & -1.93443700 & -0.03993100 \\
\hline C & 0.83686200 & -0.55876000 & 0.05329400 \\
\hline $\mathrm{C}$ & 1.36411500 & -1.23663500 & 1.18105100 \\
\hline C & 1.69326800 & -0.61293500 & -1.0838470 \\
\hline C & 2.57989900 & -1.90410400 & 1.1852270 \\
\hline $\mathrm{C}$ & 2.91330000 & -1.26704100 & -1.0950390 \\
\hline $\mathrm{C}$ & 3.36587500 & -1.93452800 & 0.0396400 \\
\hline $\mathrm{F}$ & -0.65751700 & -1.29709700 & -2.3250740 \\
\hline $\mathrm{F}$ & -1.41058400 & 0.09977300 & 2.1986860 \\
\hline $\mathrm{F}$ & -3.69339600 & -1.22109100 & 2.1875480 \\
\hline $\mathrm{F}$ & -4.54156700 & -2.57534400 & -0.0328970 \\
\hline $\mathrm{F}$ & -2.99519100 & -2.53599900 & -2.2945690 \\
\hline $\mathrm{F}$ & 0.65743800 & -1.29765300 & 2.3249330 \\
\hline $\mathrm{F}$ & 1.41053600 & 0.10025300 & -2.1985000 \\
\hline $\mathrm{F}$ & 3.69331600 & -1.22067100 & -2.1876730 \\
\hline $\mathrm{F}$ & 2.99508000 & -2.53660000 & 2.2941400 \\
\hline $\mathrm{F}$ & 4.54145500 & -2.57546000 & 0.0324590 \\
\hline $\mathrm{N}$ & -1.64558300 & 2.84417700 & -0.2403140 \\
\hline $\mathrm{N}$ & 1.64566800 & 2.84402400 & 0.2409370 \\
\hline C & 2.58579400 & 3.51379900 & 0.34271700 \\
\hline C & -2.58552200 & 3.51416300 & -0.34241600 \\
\hline C & -3.76813000 & 4.35313300 & -0.47064400 \\
\hline $\mathrm{H}$ & -4.65415600 & 3.72652600 & -0.6002210 \\
\hline $\mathrm{H}$ & -3.88839600 & 4.96276800 & 0.4283470 \\
\hline $\mathrm{H}$ & -3.66363900 & 5.01035000 & -1.3375100 \\
\hline C & 3.76867400 & 4.35246600 & 0.4704150 \\
\hline $\mathrm{H}$ & 3.66564400 & 5.00829500 & 1.3385080 \\
\hline $\mathrm{H}$ & 4.65485100 & 3.72556400 & 0.5975040 \\
\hline $\mathrm{H}$ & 3.88754500 & 4.96354400 & -0.427780 \\
\hline
\end{tabular}

\section{3) $\left[\mathrm{Pd}(\mathrm{MeCN})_{2}\right]$}

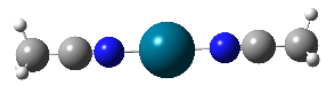


Number of imaginary frequencies $=0$

$\mathrm{E}_{\text {total }}=-393.51885011 \mathrm{a} . \mathrm{u}$

$\mathrm{G}_{\text {correction }}=0.057706 \mathrm{a} . \mathrm{u}$

\section{Cartesian coordinates:}

$\begin{array}{lrrr}\mathrm{Pd} & 0.00000000 & -0.00147000 & 0.00005400 \\ \mathrm{~N} & -1.98346900 & -0.00081700 & 0.00016000 \\ \mathrm{~N} & 1.98347100 & -0.00085600 & -0.00003200 \\ \mathrm{C} & 3.14351400 & 0.00090000 & -0.00004000 \\ \mathrm{C} & -3.14351400 & 0.00094400 & 0.00001400 \\ \mathrm{C} & -4.59811600 & 0.00354000 & -0.00019400 \\ \mathrm{H} & -4.96854400 & 0.65999000 & -0.79197800 \\ \mathrm{H} & -4.97178800 & -1.00923300 & -0.17235300 \\ \mathrm{H} & -4.96951200 & 0.36199100 & 0.96348100 \\ \mathrm{C} & 4.59811500 & 0.00357800 & -0.00012200 \\ \mathrm{H} & 4.97187700 & -1.02320900 & -0.03201200 \\ \mathrm{H} & 4.96882600 & 0.54560700 & -0.87409400 \\ \mathrm{H} & 4.96913300 & 0.49042800 & 0.90563700\end{array}$

4) $\left(\mathrm{C}_{6} \mathrm{~F}_{5}\right)_{2}$

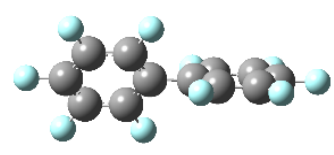

Number of imaginary frequencies $=0$

$\mathrm{E}_{\text {total }}=-1455.61804677 \mathrm{a} . \mathrm{u}$

$\mathrm{G}_{\text {correction }}=0.052999 \mathrm{a} . \mathrm{u}$

\section{Cartesian coordinates:}

C
C
C
C
C
C
C
C
C
C
C
C
F
F
F
F
F
F
F
F
F
F
$-0.73798700$

$-1.46513700$

$-1.46516000$

$-2.85502500$

$-2.85505200$

$-3.55159400$

0.73798900

1.46516000

1.46513700

2.85505700

2.85502100

3.55159100

$-0.82257300$

$-0.82263200$

$-3.52380700$

$-4.88636100$

$-3.52376300$

0.82263500

0.82256700

3. 52377300

3. 52380200

4.88636000
0.00002600

$-1.06525900$

1.06528800

$-1.07765000$

1.07764900

$-0.00000400$

0.00002600

1.06527800

$-1.06526000$

1.07764400

$-1.07764700$

$-0.00000300$

$-2.10602500$

2.10604200

2.11044900

$-0.00002700$

$-2.11047500$

2.10603500

$-2.10600900$

$-2.11045600$

2.11044500

$-0.00003700$
0.00000400

0.53906800

$-0.53907200$

0.53886400

$-0.53886500$

0.00000500

0.00000200

0.53908600

$-0.53907500$

0.53888100

$-0.53887100$

0.00000100

1.08483000

$-1.08489600$

$-1.06384500$

0.00000100

1.06381700

1.08491300

$-1.08485600$

$-1.06383800$

1.06386400

$-0.00000800$ 


\section{5) $\left[\mathrm{Pd}(\mathrm{MeCN})\left(\mathrm{C}_{6} \mathrm{~F}_{5}\right)_{2}\right](\mathbf{5})$}

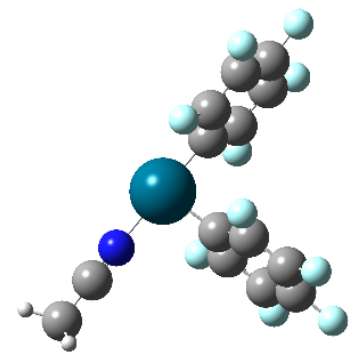

Number of imaginary frequencies $=0$

$E_{\text {total }}=-1716.34277731$ a.u

$\mathrm{G}_{\text {correction }}=0.085593 \mathrm{a} . \mathrm{u}$

\section{Cartesian coordinates:}

$\begin{array}{lrrr}\text { Pd } & 0.39189600 & -1.61610800 & -0.16760200 \\ \text { C } & -1.40001400 & -0.73594900 & -0.26405500 \\ \text { C } & -2.35464700 & -1.01442600 & 0.70898800 \\ \text { C } & -1.78343300 & 0.15008500 & -1.26489800 \\ \text { C } & -3.63042700 & -0.45903400 & 0.69790100 \\ \text { C } & -3.04963900 & 0.72744300 & -1.31421200 \\ \text { C } & -3.97790100 & 0.41989100 & -0.32400800 \\ \text { C } & 1.11052300 & 0.19587000 & 0.14330100 \\ \text { C } & 1.91366000 & 0.80029400 & -0.81610900 \\ \text { C } & 0.86883400 & 0.91023400 & 1.30958400 \\ \text { C } & 2.46586200 & 2.06471600 & -0.63061600 \\ \text { C } & 1.40641000 & 2.17699900 & 1.52629900 \\ \text { C } & 2.20878400 & 2.75702700 & 0.54895600 \\ \text { F } & -2.06087800 & -1.86074100 & 1.72637000 \\ \text { F } & -0.91825200 & 0.49086300 & -2.24852600 \\ \text { F } & -3.38827200 & 1.57255100 & -2.30265000 \\ \text { F } & -5.20347200 & 0.96287700 & -0.35759700 \\ \text { F } & -4.52713100 & -0.75714500 & 1.65340400 \\ \text { F } & 2.18971900 & 0.16796300 & -1.97833700 \\ \text { F } & 0.09337400 & 0.39263600 & 2.28750000 \\ \text { F } & 1.16051500 & 2.84174700 & 2.66697900 \\ \text { F } & 3.24033000 & 2.62254600 & -1.57531700 \\ \text { F } & 2.73539800 & 3.97458000 & 0.74447300 \\ \text { N } & 2.28869700 & -2.50857300 & -0.05898900 \\ \text { C } & 3.35448500 & -2.95241100 & -0.00177000 \\ \text { C } & 4.69442200 & -3.51019500 & 0.06757900 \\ \text { H } & 5.13233800 & -3.29378000 & 1.04506600 \\ \text { H } & 4.64944600 & -4.59246400 & -0.07691600 \\ \text { H } & 5.31660300 & -3.06695000 & -0.71387300 \\ & & & \end{array}$

6) $\mathrm{MeCN}$

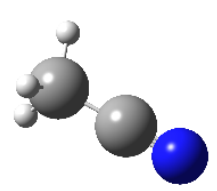

Number of imaginary frequencies $=0$ 
$\mathrm{E}_{\text {total }}=-132.77158285 \mathrm{a} \cdot \mathrm{u}$

$\mathrm{G}_{\text {correction }}=0.021432 \mathrm{a} . \mathrm{u}$

Cartesian coordinates:

$\begin{array}{lrrr}\text { C } & 0.27806500 & -0.00030500 & -0.00055000 \\ \mathrm{C} & -1.17994800 & 0.00007300 & 0.00012800 \\ \mathrm{H} & -1.55441200 & 0.10906900 & -1.02068800 \\ \mathrm{H} & -1.55393400 & -0.93856600 & 0.41643300 \\ \mathrm{H} & -1.55357900 & 0.82990400 & 0.60509900 \\ \mathrm{~N} & 1.43903100 & 0.00014100 & 0.00024100\end{array}$

\section{7) $\operatorname{TS}(5-6)$}

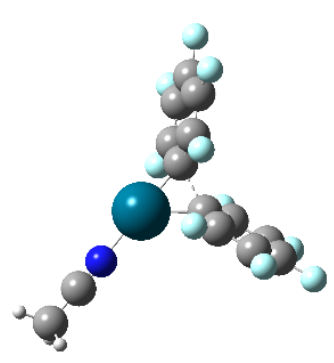

Number of imaginary frequencies $=1\left(i 249.64 \mathrm{~cm}^{-1}\right)$

$E_{\text {total }}=-1716.30592047 \mathrm{a} . \mathrm{u}$

$\mathrm{G}_{\text {correction }}=0.087126 \mathrm{a} . \mathrm{u}$

\section{Cartesian coordinates:}

$\begin{array}{lrrr}\mathrm{Pd} & 0.60653700 & 1.54468500 & -0.14541600 \\ \mathrm{C} & -1.00519900 & 0.28053900 & -0.13189100 \\ \mathrm{C} & -1.70292600 & -0.21872100 & -1.25334600 \\ \mathrm{C} & -1.84041700 & 0.67587200 & 0.94409600 \\ \mathrm{C} & -3.08330200 & -0.35701400 & -1.29502800 \\ \mathrm{C} & -3.22178200 & 0.54805600 & 0.92011400 \\ \mathrm{C} & -3.85408800 & 0.02032300 & -0.20099800 \\ \mathrm{C} & 0.58807100 & -0.52554300 & 0.10160900 \\ \mathrm{C} & 0.65199900 & -1.25364600 & 1.30539600 \\ \mathrm{C} & 1.33040400 & -1.09178300 & -0.96084700 \\ \mathrm{C} & 1.35083400 & -2.44635200 & 1.44159100 \\ \mathrm{C} & 2.03750300 & -2.27969600 & -0.84480800 \\ \mathrm{C} & 2.04351900 & -2.97554500 & 0.35968500 \\ \mathrm{~F} & -1.03480800 & -0.63946000 & -2.33992900 \\ \mathrm{~F} & -1.33065200 & 1.27806200 & 2.03897800 \\ \mathrm{~F} & -3.95905400 & 0.97314500 & 1.95679000 \\ \mathrm{~F} & -5.18451900 & -0.10040200 & -0.23513600 \\ \mathrm{~F} & -3.67848700 & -0.85714200 & -2.38749400 \\ \mathrm{~F} & -0.01090000 & -0.83024500 & 2.39601600 \\ \mathrm{~F} & 1.47032800 & -0.44201900 & -2.13612900 \\ \mathrm{~F} & 2.75388700 & -2.74340000 & -1.88053600 \\ \mathrm{~F} & 1.35022000 & -3.09555800 & 2.61551700 \\ \mathrm{~F} & 2.72065300 & -4.12317300 & 0.47929700 \\ \mathrm{~N} & 2.26852000 & 2.82424100 & -0.07730400 \\ \mathrm{C} & 3.16727500 & 3.55254400 & -0.02328800 \\ \mathrm{C} & 4.30166300 & 4.45919900 & 0.04838800\end{array}$




\section{8) Intermediate-6}

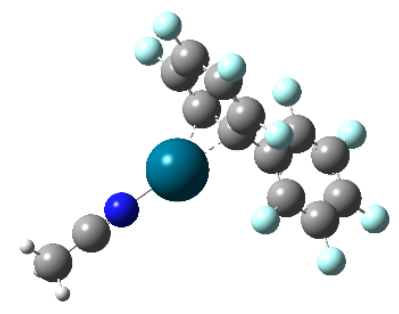

Number of imaginary frequencies $=0$

$$
\mathrm{E}_{\text {total }}=-1716.34874631 \mathrm{a} . \mathrm{u}
$$

$\mathrm{G}_{\text {correction }}=0.083376 \mathrm{a} . \mathrm{u}$

\section{Cartesian coordinates:}

Pd
C
C
C
C
C
C
C
C
C
C
C
C
F
F
F
F
F
F
F
F
F
F
N
C
C
H
H
H

$$
\begin{array}{r}
1.39799300 \\
-0.01570900 \\
0.37724000 \\
0.79071000 \\
1.42726700 \\
1.84977400 \\
2.17077800 \\
-1.42456900 \\
-2.33622100 \\
-1.90918700 \\
-3.65852600 \\
-3.22767800 \\
-4.10610000 \\
-0.35515600 \\
0.27280300 \\
2.53842800 \\
3.21842000 \\
1.78824900 \\
-1.94971000 \\
-1.09069100 \\
-3.65536900 \\
-4.50390600 \\
-5.37521100 \\
2.68273100 \\
3.38035200 \\
4.26044900 \\
4.00195800 \\
5.29809000 \\
4.15096200
\end{array}
$$

$$
\begin{array}{r}
-0.08849400 \\
0.18777000 \\
1.38180300 \\
-0.98271800 \\
1.40833000 \\
-0.94004400 \\
0.23497500 \\
0.07465400 \\
-0.75106200 \\
0.81744900 \\
-0.87030100 \\
0.72057200 \\
-0.12909500 \\
2.49268100 \\
-2.21960200 \\
-2.06240500 \\
0.29900700 \\
2.54719300 \\
-1.44678500 \\
1.64151200 \\
1.43576400 \\
-1.67619300 \\
-0.22980300 \\
-0.33174100 \\
-0.42483100 \\
-0.54596600 \\
0.21302100 \\
-0.40419600 \\
-1.53744800
\end{array}
$$

\section{9) $\left[\mathrm{Pd}(\mathrm{THF})_{2}\left(\mathrm{C}_{6} \mathrm{~F}_{5}\right)_{2}\right](7)$}




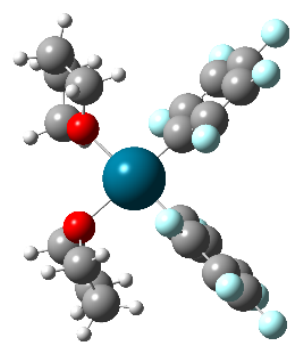

Number of imaginary frequencies $=0$

$$
\mathrm{E}_{\text {total }}=-2048.56768273 \mathrm{a} . \mathrm{u}
$$

$\mathrm{G}_{\text {correction }}=0.270449 \mathrm{a} . \mathrm{u}$

\section{Cartesian coordinates:}

\begin{tabular}{|c|c|c|c|}
\hline $\mathrm{Pd}$ & -0.10781500 & 0.96359500 & -0.29143900 \\
\hline $\mathrm{C}$ & -1.27552700 & -0.62958800 & -0.07896500 \\
\hline C & -1.43704800 & -1.59758400 & -1.06339700 \\
\hline C & -2.04170200 & -0.79247200 & 1.06974600 \\
\hline $\mathrm{C}$ & -2.32053700 & -2.66654200 & -0.93311600 \\
\hline $\mathrm{C}$ & -2.93674800 & -1.84493000 & 1.24083800 \\
\hline $\mathrm{C}$ & -3.07461000 & -2.79114200 & 0.22976200 \\
\hline C & 1.44488500 & -0.25623700 & -0.06050100 \\
\hline $\mathrm{C}$ & 1.85979200 & -0.69511600 & 1.19057700 \\
\hline $\mathrm{C}$ & 2.21577300 & -0.67193800 & -1.13959500 \\
\hline $\mathrm{C}$ & 2.99042300 & -1.48485300 & 1.38001500 \\
\hline $\mathrm{C}$ & 3.35185300 & -1.46447800 & -0.99769000 \\
\hline $\mathrm{C}$ & 3.73978000 & -1.87635000 & 0.27404100 \\
\hline $\mathrm{F}$ & -0.74038400 & -1.51847900 & -2.22127800 \\
\hline $\mathrm{F}$ & -1.95440100 & 0.10402100 & 2.08625600 \\
\hline $\mathrm{F}$ & -3.66515900 & -1.95912900 & 2.3643120 \\
\hline $\mathrm{F}$ & -3.93218300 & -3.81281300 & 0.3726880 \\
\hline $\mathrm{F}$ & -2.45700000 & -3.57431900 & -1.91421300 \\
\hline $\mathrm{F}$ & 1.18143300 & -0.32104900 & 2.30434500 \\
\hline $\mathrm{F}$ & 1.90673900 & -0.26894000 & -2.3980360 \\
\hline $\mathrm{F}$ & 4.07825100 & -1.83173400 & -2.0662970 \\
\hline $\mathrm{F}$ & 3.36839400 & -1.87044600 & 2.6100990 \\
\hline $\mathrm{F}$ & 4.83195300 & -2.63820900 & 0.4333420 \\
\hline 0 & -1.83152100 & 2.28512300 & -0.53994100 \\
\hline $\mathrm{C}$ & -2.43695200 & 2.91331400 & 0.6327410 \\
\hline C & -2.85972600 & 1.94372200 & -1.51567200 \\
\hline C & -3.93956400 & 2.72850500 & 0.45052900 \\
\hline $\mathrm{H}$ & -2.04043300 & 2.42124200 & 1.51996300 \\
\hline $\mathrm{H}$ & -2.14414000 & 3.96795600 & 0.62706100 \\
\hline C & -4.08818000 & 2.73175000 & -1.07805300 \\
\hline $\mathrm{H}$ & -2.47289200 & 2.20985200 & -2.50091400 \\
\hline $\mathrm{H}$ & -3.03332400 & 0.86471000 & -1.47053500 \\
\hline $\mathrm{H}$ & -4.51340800 & 3.51638800 & 0.94291200 \\
\hline $\mathrm{H}$ & -4.25147500 & 1.76308800 & 0.86214300 \\
\hline $\mathrm{H}$ & -4.04943500 & 3.75493800 & -1.46511300 \\
\hline $\mathrm{H}$ & -5.01490400 & 2.26629900 & -1.42059200 \\
\hline 0 & 1.13060000 & 2.79203800 & -0.4058740 \\
\hline $\mathrm{C}$ & 1.31986000 & 3.43111200 & 0.88272800 \\
\hline $\mathrm{C}$ & 2.45450100 & 2.74359700 & -1.0023040 \\
\hline C & 2.61088100 & 2.82274500 & 1.43297300 \\
\hline
\end{tabular}




$\begin{array}{lrrr}\mathrm{H} & 1.41382900 & 4.51020700 & 0.71167000 \\ \mathrm{H} & 0.43187300 & 3.23331200 & 1.48260500 \\ \mathrm{C} & 3.42807900 & 2.47471500 & 0.15964500 \\ \mathrm{H} & 2.43434200 & 1.97440200 & -1.77085300 \\ \mathrm{H} & 2.63891900 & 3.71949700 & -1.46501900 \\ \mathrm{H} & 2.38209600 & 1.92105100 & 2.00372200 \\ \mathrm{H} & 3.13330000 & 3.52089400 & 2.09044800 \\ \mathrm{H} & 3.74015900 & 1.42953200 & 0.17504000 \\ \mathrm{H} & 4.32215500 & 3.09445300 & 0.06341100\end{array}$

\section{0) $\quad \mathbf{T S}(7-8)$}

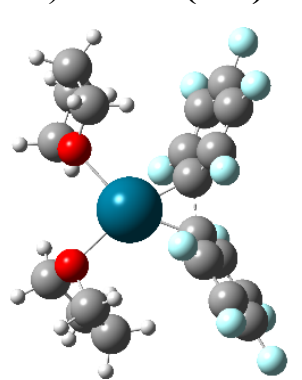

Number of imaginary frequencies $=1\left(i 111.30 \mathrm{~cm}^{-1}\right)$

$E_{\text {total }}=-2048.51462478$ a.u

$\mathrm{G}_{\text {correction }}=0.269660 \mathrm{a} . \mathrm{u}$

\section{Cartesian coordinates:}

$\begin{array}{lrrr}\mathrm{Pd} & -0.04239100 & 1.04020300 & -0.46326300 \\ \mathrm{C} & -0.63838000 & -0.88648900 & -0.00607500 \\ \mathrm{C} & -1.10708300 & -1.86040800 & -0.92441500 \\ \mathrm{C} & -1.50341500 & -0.70897500 & 1.11319700 \\ \mathrm{C} & -2.28977800 & -2.56958100 & -0.76727100 \\ \mathrm{C} & -2.69491200 & -1.39541100 & 1.27497900 \\ \mathrm{C} & -3.09941300 & -2.34413300 & 0.34005600 \\ \mathrm{C} & 1.03486500 & -0.68020300 & 0.04222500 \\ \mathrm{C} & 1.64269500 & -0.97151800 & 1.28892000 \\ \mathrm{C} & 1.89010800 & -0.92113200 & -1.06998800 \\ \mathrm{C} & 2.93264500 & -1.46238700 & 1.42537700 \\ \mathrm{C} & 3.18300600 & -1.40535900 & -0.95410500 \\ \mathrm{C} & 3.71562400 & -1.69504300 & 0.29939400 \\ \mathrm{~F} & -0.38153800 & -2.16452800 & -2.01548600 \\ \mathrm{~F} & -1.27589100 & 0.26121500 & 2.03113000 \\ \mathrm{~F} & -3.50914700 & -1.09079100 & 2.30088200 \\ \mathrm{~F} & -4.25008200 & -3.00915600 & 0.49470600 \\ \mathrm{~F} & -2.65365700 & -3.47972000 & -1.68300300 \\ \mathrm{~F} & 0.95239500 & -0.80386500 & 2.43232700 \\ \mathrm{~F} & 1.51904200 & -0.56147000 & -2.32170700 \\ \mathrm{~F} & 3.95169500 & -1.54613600 & -2.04563500 \\ \mathrm{~F} & 3.42753300 & -1.72499200 & 2.64440400 \\ \mathrm{~F} & 4.96086600 & -2.16975700 & 0.41948500 \\ \mathrm{O} & -2.04679300 & 2.23134500 & -0.79174500 \\ \mathrm{C} & -2.58605800 & 2.85644100 & 0.40567500 \\ \mathrm{C} & -3.08351600 & 1.45248800 & -1.44759500 \\ \mathrm{C} & -3.92989000 & 2.17758700 & 0.65797900 \\ \mathrm{H} & -1.86997700 & 2.71129400 & 1.21618600 \\ \mathrm{~F} & & & \end{array}$




$\begin{array}{lrrr}\mathrm{H} & -2.70103400 & 3.92870000 & 0.20944900 \\ \mathrm{C} & -4.39322000 & 1.84650600 & -0.76815200 \\ \mathrm{H} & -3.04893600 & 1.67953500 & -2.51577300 \\ \mathrm{H} & -2.86127900 & 0.39009600 & -1.30319400 \\ \mathrm{H} & -4.62462700 & 2.82629900 & 1.19639800 \\ \mathrm{H} & -3.79248500 & 1.26357100 & 1.24315300 \\ \mathrm{H} & -4.82425800 & 2.73312200 & -1.24415800 \\ \mathrm{H} & -5.13213400 & 1.04268700 & -0.80508500 \\ \mathrm{O} & 1.23718400 & 2.97254700 & -0.68915200 \\ \mathrm{C} & 1.34910300 & 3.65561400 & 0.57869600 \\ \mathrm{C} & 2.60039700 & 2.67031100 & -1.06454200 \\ \mathrm{C} & 2.40093300 & 2.85835300 & 1.35762100 \\ \mathrm{H} & 1.67996500 & 4.68615400 & 0.39274700 \\ \mathrm{H} & 0.35803700 & 3.67070100 & 1.03252700 \\ \mathrm{C} & 3.30908600 & 2.27116200 & 0.24293800 \\ \mathrm{H} & 2.56407600 & 1.88442000 & -1.81836400 \\ \mathrm{H} & 3.04478800 & 3.57347000 & -1.50178100 \\ \mathrm{H} & 1.92039300 & 2.05432000 & 1.92041700 \\ \mathrm{H} & 2.94843500 & 3.48974200 & 2.06096800 \\ \mathrm{H} & 3.37470600 & 1.18673100 & 0.33300600 \\ \mathrm{H} & 4.32345200 & 2.67460000 & 0.27735600\end{array}$

\section{1) Intermediate-8}

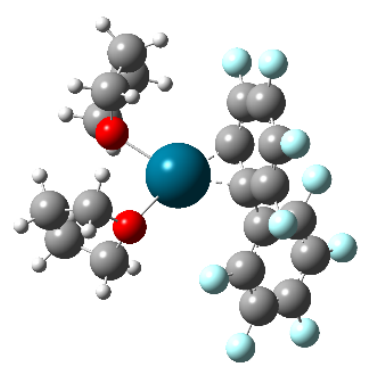

Number of imaginary frequencies $=0$

$\mathrm{E}_{\text {total }}=-2048.55171606 \mathrm{a} . \mathrm{u}$

$\mathrm{G}_{\text {correction }}=0.269902 \mathrm{a} . \mathrm{u}$

\section{Cartesian coordinates:}

$\begin{array}{lrrr}\text { Pd } & 0.98133300 & 0.35790100 & 0.08063600 \\ C & -0.73361100 & -0.81744600 & 0.45191900 \\ C & -0.66398700 & -1.42537400 & 1.75593500 \\ C & 0.15870500 & -1.37414400 & -0.56900500 \\ C & 0.14029300 & -2.49266100 & 2.03162400 \\ C & 0.91880300 & -2.55759300 & -0.25718300 \\ C & 0.94449200 & -3.06509800 & 1.00771200 \\ C & -2.03947700 & -0.21277900 & 0.05827500 \\ C & -2.94292400 & -0.92700800 & -0.73470500 \\ C & -2.44349100 & 1.05331200 & 0.48587600 \\ C & -4.16296400 & -0.39098400 & -1.13372600 \\ C & -3.66107900 & 1.60867900 & 0.10748500 \\ C & -4.52468000 & 0.88331800 & -0.70797300 \\ \text { F } & -1.46225400 & -0.93151500 & 2.72310500 \\ \text { F } & -0.23365400 & -1.31380900 & -1.89425800 \\ \text { F } & 1.64088300 & -3.13125000 & -1.24095200 \\ \text { F } & 1.75171400 & -4.09041800 & 1.32904200\end{array}$


0.22884500

$-2.65036900$

$-1.64510400$

$-4.00775500$

$-4.99829400$

$-5.69832800$

3.01608200

2. 97851400

4.08193500

3.17840700

2.02038600

3. 79424300

4.13286100

5.01667900

3.84317600

3.59099100

2. 22371600

5.15324100

3.79319000

1.37609000

2.73334100

0.86874300

3.28073100

2. 70955500

3.27465600

2. 02759100

$-0.03217500$

0.60890300

4.01382500

3.75773600

2. 01629200

1.97254100
$-3.00385200$

$-2.17680400$

1. 78853400

2.83296700

$-1.10042100$

1.40664900

0.84990400

0.90134300

$-0.06883500$

$-0.54816200$

1.33689100

1.54846400

$-1.11421600$

0.50075500

$-0.48822600$

$-0.62101100$

$-1.07683200$

$-1.21990600$

$-2.09208500$

2. 31539100

2. 61725900

3. 51958600

3.60578800

3.06701400

1. 67277700

4.02995800

3. 25313900

4.23503400

3. 12295600

4.46045800

3.53543900

5.10894800
3.27428300

$-1.12470000$

1.27711700

0.52610300

$-1.90329500$

$-1.07596100$

$-0.90756200$

$-2.34886400$

$-0.56872900$

$-2.78534900$

$-2.63493400$

$-2.69938400$

$-1.70374500$

$-0.49961300$

0.40933400

$-3.79428000$

$-2.76193400$

$-2.07964300$

$-1.36215300$

1.25483500

1.64657900

0.64395200

0.59536400

2.64690400

1.69246400

$-0.21166800$

0.09339600

1. 43639200

$-0.05170100$

1.08148800

$-1.18609000$

$-0.37469100$

\section{2) $\left[\mathrm{Pd}(\mathrm{THF})_{2}\right](\mathbf{9})$}

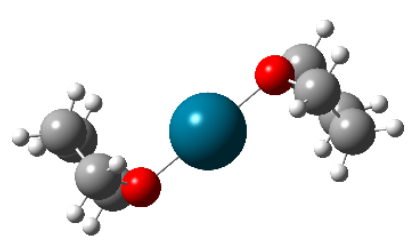

Number of imaginary frequencies $=0$

$\mathrm{E}_{\text {total }}=-592.89886036 \mathrm{a} \cdot \mathrm{u}$

$\mathrm{G}_{\text {correction }}=0.194714 \mathrm{a} . \mathrm{u}$

\section{Cartesian coordinates:}

$\begin{array}{lr}\mathrm{Pd} & 0.00000100 \\ \mathrm{O} & -1.95177500 \\ \mathrm{C} & -2.64541200 \\ \mathrm{C} & -2.88208400 \\ \mathrm{C} & -3.27483300 \\ \mathrm{H} & -1.91278800 \\ \mathrm{H} & -3.41180800 \\ \mathrm{C} & -3.45501000\end{array}$
0.17509400
0.18539700
$-1.08594400$
1.12634700
$-1.10710100$
$-1.86893000$
$-1.09507600$
0.39869600




$\begin{array}{lrrr}\mathrm{H} & -3.66152700 & 1.33881500 & 1.06861200 \\ \mathrm{H} & -2.32712900 & 2.03633800 & 0.10289700 \\ \mathrm{H} & -4.21930100 & -1.65636600 & -0.55649700 \\ \mathrm{H} & -2.59522100 & -1.58256000 & -1.26420500 \\ \mathrm{H} & -4.50045000 & 0.66885300 & -1.06563200 \\ \mathrm{H} & -2.88567000 & 0.65658200 & -1.79492800 \\ \mathrm{O} & 1.95179700 & 0.18538200 & -0.92084300 \\ \mathrm{C} & 2.88210200 & 1.12634100 & -0.32499800 \\ \mathrm{C} & 2.64542100 & -1.08596600 & -0.84510300 \\ \mathrm{C} & 3.45492900 & 0.39872800 & 0.89897800 \\ \mathrm{H} & 3.66158800 & 1.33875100 & -1.06859700 \\ \mathrm{H} & 2.32715200 & 2.03635200 & -0.10298700 \\ \mathrm{C} & 3.27486300 & -1.10708000 & 0.55305800 \\ \mathrm{H} & 1.91277900 & -1.86894600 & -1.03393700 \\ \mathrm{H} & 3.41180500 & -1.09514500 & -1.63149800 \\ \mathrm{H} & 2.88545800 & 0.65659100 & 1.79489800 \\ \mathrm{H} & 4.50033500 & 0.66893700 & 1.06581800 \\ \mathrm{H} & 2.59531500 & -1.58260800 & 1.26425400 \\ \mathrm{H} & 4.21938200 & -1.65626000 & 0.55650400\end{array}$

\section{3) $\quad\left(\mathrm{C}_{6} \mathrm{~F}_{5}\right)_{2}$}

\section{每}

Number of imaginary frequencies $=0$

$$
\mathrm{E}_{\text {total }}=-1455.61737333 \mathrm{a} . \mathrm{u}
$$

$\mathrm{G}_{\text {correction }}=0.053037 \mathrm{a} . \mathrm{u}$

\section{Cartesian coordinates:}

$$
\begin{aligned}
& C \\
& C \\
& C \\
& C \\
& C \\
& C \\
& C \\
& C \\
& C \\
& C \\
& C \\
& C \\
& C \\
& C \\
& C \\
& \text { F } \\
& \text { F } \\
& \text { F } \\
& \text { F } \\
& \text { F } \\
& \text { F } \\
& \text { F } \\
& \text { F } \\
& \text { F }
\end{aligned}
$$$$
\text { F }
$$$$
\text { F }
$$$$
\text { F }
$$

0.73801000

1.46499400

1.46501700

2.85504900

2.85507700

3.55160500

$-0.73801100$

$-1.46501700$

$-1.46499500$

$-2.85508100$

$-2.85504600$

$-3.55160300$

0.82214600

0.82220400

3.52374200

4.88606600

3.52369900

$-0.82220700$

$-0.82214000$

$-3.52370800$

$-3.52373600$
0.00002600

$-1.06537200$

1.06540000

$-1.07785900$

1.07785800

$-0.00000400$

0.00002600

1.06539000

$-1.06537300$

1.07785300

$-1.07785600$

$-0.00000300$

$-2.10543600$

2. 10545200

2.11025800

$-0.00002700$

$-2.11028400$

2.10544500

$-2.10542000$

$-2.11026400$

2.11025400
$-0.00000400$

$-0.53935700$

0.53936100

$-0.53905400$

0.53905400

$-0.00000500$

$-0.00000100$

$-0.53937500$

0.53936500

$-0.53907100$

0.53906100

$-0.00000100$

$-1.08546600$

1.08553100

1.06400800

$-0.00000100$

$-1.06398100$

$-1.08554700$

1.08549200

1.06400200

$-1.06402800$

\section{S63}


14) $\left[\mathrm{Pd}(\mathrm{THF})\left(\mathrm{C}_{6} \mathrm{~F}_{5}\right)_{2}\right](\mathbf{1 0})$

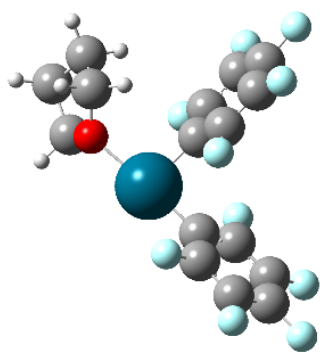

Number of imaginary frequencies $=0$

$E_{\text {total }}=-1816.05032820 \mathrm{a} . \mathrm{u}$

$\mathrm{G}_{\text {correction }}=0.157324 \mathrm{a} . \mathrm{u}$

\section{Cartesian coordinates:}

$\begin{array}{lrrr}\mathrm{Pd} & 0.22582100 & -1.42524500 & -0.07732900 \\ \mathrm{C} & -1.61754600 & -0.74518900 & 0.17999700 \\ \mathrm{C} & -2.02824300 & -0.01061900 & 1.28779100 \\ \mathrm{C} & -2.58918200 & -1.01098100 & -0.78065800 \\ \mathrm{C} & -3.33808800 & 0.43428300 & 1.44830100 \\ \mathrm{C} & -3.90779500 & -0.58455100 & -0.65762800 \\ \mathrm{C} & -4.28225900 & 0.14531000 & 0.46731000 \\ \mathrm{C} & 0.81304100 & 0.45246200 & -0.16653100 \\ \mathrm{C} & 0.44790500 & 1.30478200 & -1.20277000 \\ \mathrm{C} & 1.69096800 & 0.95919600 & 0.78518600 \\ \mathrm{C} & 0.94117900 & 2.60399000 & -1.30324200 \\ \mathrm{C} & 2.20646300 & 2.24973900 & 0.71235700 \\ \mathrm{C} & 1.82482600 & 3.07858500 & -0.33867100 \\ \mathrm{~F} & -1.15001700 & 0.30420000 & 2.26715800 \\ \mathrm{~F} & -2.26791800 & -1.71104600 & -1.89571800 \\ \mathrm{~F} & -4.81911300 & -0.86473100 & -1.60360900 \\ \mathrm{~F} & -5.54858200 & 0.56125900 & 0.60860200 \\ \mathrm{~F} & -3.70248300 & 1.13417100 & 2.53518200 \\ \mathrm{~F} & -0.39697100 & 0.89064000 & -2.17123500 \\ \mathrm{~F} & 2.10025300 & 0.19096800 & 1.82286500 \\ \mathrm{~F} & 3.06404300 & 2.70071100 & 1.64094800 \\ \mathrm{~F} & 0.57800000 & 3.39948300 & -2.32091000 \\ \mathrm{~F} & 2.31156200 & 4.32378600 & -0.42501200 \\ \mathrm{O} & 2.21064600 & -2.26471500 & -0.36305700 \\ \mathrm{C} & 3.21228300 & -1.60899800 & -1.20044900 \\ \mathrm{C} & 2.85617300 & -2.85277200 & 0.81569500 \\ \mathrm{~F} & & & \end{array}$

\section{S64}




$\begin{array}{lrrr}\mathrm{C} & 4.39767000 & -1.38578500 & -0.27390100 \\ \mathrm{H} & 3.45563100 & -2.28675000 & -2.02521900 \\ \mathrm{H} & 2.76656400 & -0.69634500 & -1.59369200 \\ \mathrm{C} & 4.35518200 & -2.64564800 & 0.60368000 \\ \mathrm{H} & 2.48898400 & -2.31690200 & 1.69314900 \\ \mathrm{H} & 2.55453800 & -3.90070000 & 0.86841300 \\ \mathrm{H} & 4.23697200 & -0.49129900 & 0.33526300 \\ \mathrm{H} & 5.33560500 & -1.27449800 & -0.82193900 \\ \mathrm{H} & 4.88329900 & -2.52624300 & 1.55193200 \\ \mathrm{H} & 4.79104600 & -3.49605300 & 0.07045100\end{array}$

15) THF

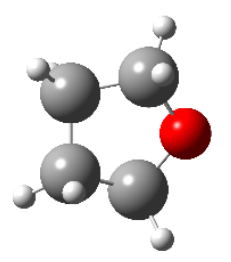

Number of imaginary frequencies $=0$

$\mathrm{E}_{\text {total }}=-232.47855000 \mathrm{a} \cdot \mathrm{u}$

$\mathrm{G}_{\text {correction }}=0.088513 \mathrm{a} . \mathrm{u}$

Cartesian coordinates:

$\begin{array}{lrrr}\mathrm{C} & -1.16703200 & -0.42677400 & -0.13274100 \\ \mathrm{O} & -0.00030300 & -1.25435600 & -0.00064300 \\ \mathrm{C} & 1.16656400 & -0.42742800 & 0.13362200 \\ \mathrm{C} & 0.73167200 & 0.99417700 & -0.23125900 \\ \mathrm{C} & -0.73086600 & 0.99487500 & 0.23081300 \\ \mathrm{H} & -1.53186900 & -0.47331900 & -1.16931600 \\ \mathrm{H} & -1.95290400 & -0.81779100 & 0.52237400 \\ \mathrm{H} & 1.52943300 & -0.47356200 & 1.17094100 \\ \mathrm{H} & 1.95341300 & -0.81926200 & -0.51979400 \\ \mathrm{H} & 1.34384500 & 1.75882500 & 0.25300600 \\ \mathrm{H} & 0.78793300 & 1.14476200 & -1.31472200 \\ \mathrm{H} & -0.78693100 & 1.14656400 & 1.31412900 \\ \mathrm{H} & -1.34253000 & 1.75952800 & -0.25408200\end{array}$

16) $\operatorname{TS}(10-11)$

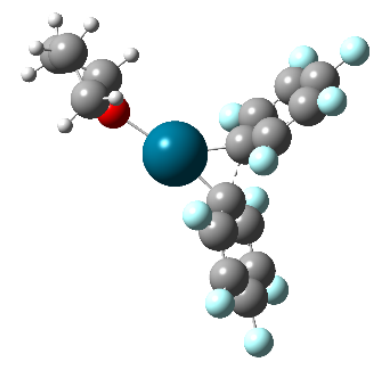

Number of imaginary frequencies $=1\left(i 214.60 \mathrm{~cm}^{-1}\right)$

$\mathrm{E}_{\text {total }}=-1816.00895341 \mathrm{a} . \mathrm{u}$

$\mathrm{G}_{\text {correction }}=0.155601 \mathrm{a} . \mathrm{u}$ 


\section{Cartesian coordinates:}

\begin{tabular}{|c|c|c|c|}
\hline $\mathrm{Pd}$ & -0.87768100 & -0.96888500 & -0.13783100 \\
\hline $\mathrm{C}$ & 1.11394700 & -0.48845700 & -0.13819900 \\
\hline $\mathrm{C}$ & 1.95620600 & -0.31872200 & -1.25993100 \\
\hline $\mathrm{C}$ & 1.70864500 & -1.21390300 & 0.92744200 \\
\hline $\mathrm{C}$ & 3.26446500 & -0.77948200 & -1.31219800 \\
\hline $\mathrm{C}$ & 3.01318200 & -1.68459500 & 0.89207700 \\
\hline $\mathrm{C}$ & 3.80667700 & -1.46166200 & -0.22884200 \\
\hline $\mathrm{C}$ & 0.01209200 & 0.88028600 & 0.10945200 \\
\hline $\mathrm{C}$ & 0.25211400 & 1.56913200 & 1.31709700 \\
\hline $\mathrm{C}$ & -0.45752700 & 1.70451900 & -0.94386200 \\
\hline $\mathrm{C}$ & 0.08065800 & 2.93934900 & 1.46412300 \\
\hline $\mathrm{C}$ & -0.63878000 & 3.07381700 & -0.81514200 \\
\hline $\mathrm{C}$ & -0.35944200 & 3.70642500 & 0.39203600 \\
\hline $\mathrm{F}$ & 1.52829900 & 0.36274200 & -2.33563900 \\
\hline $\mathrm{F}$ & 0.99689200 & -1.55586200 & 2.02244900 \\
\hline $\mathrm{F}$ & 3.50407500 & -2.39537700 & 1.91824100 \\
\hline $\mathrm{F}$ & 5.06289900 & -1.91626300 & -0.27216400 \\
\hline $\mathrm{F}$ & 4.01218700 & -0.56215600 & -2.40385300 \\
\hline $\mathrm{F}$ & 0.71175900 & 0.91344200 & 2.39699300 \\
\hline $\mathrm{F}$ & -0.84518900 & 1.17527000 & -2.12456700 \\
\hline $\mathrm{F}$ & -1.12532800 & 3.79013800 & -1.84009000 \\
\hline $\mathrm{F}$ & 0.34930800 & 3.52854000 & 2.63870900 \\
\hline $\mathrm{F}$ & -0.53142400 & 5.02623800 & 0.52449500 \\
\hline 0 & -2.89338600 & -1.81238300 & -0.14249100 \\
\hline $\mathrm{C}$ & -3.55900000 & -2.25767000 & 1.07688700 \\
\hline $\mathrm{C}$ & -3.87377600 & -1.48283100 & -1.16439500 \\
\hline C & -5.05129600 & -2.05191000 & 0.82079600 \\
\hline $\mathrm{H}$ & -3.30341300 & -3.31141300 & 1.22491500 \\
\hline $\mathrm{H}$ & -3.16878400 & -1.67011800 & 1.91035600 \\
\hline $\mathrm{C}$ & -5.15111600 & -2.17656200 & -0.70772600 \\
\hline $\mathrm{H}$ & -3.98980800 & -0.39375000 & -1.19824400 \\
\hline $\mathrm{H}$ & -3.48053500 & -1.83517900 & -2.11961700 \\
\hline $\mathrm{H}$ & -5.35932300 & -1.05109700 & 1.13970300 \\
\hline $\mathrm{H}$ & -5.66202300 & -2.78448800 & 1.35262800 \\
\hline $\mathrm{H}$ & -6.04617900 & -1.70430400 & -1.1182220 \\
\hline $\mathrm{H}$ & -5.14313300 & -3.22866500 & -1.0102510 \\
\hline
\end{tabular}

17) Intermediate-11

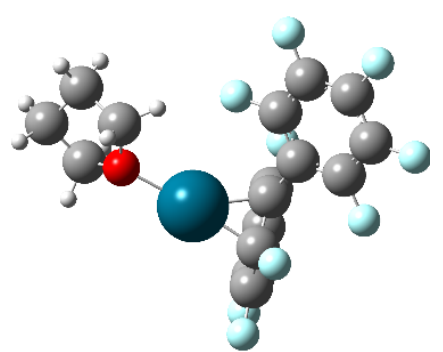

Number of imaginary frequencies $=0$

$\mathrm{E}_{\text {total }}=-1816.05127966 \mathrm{a} . \mathrm{u}$

$\mathrm{G}_{\text {correction }}=0.157863 \mathrm{a} . \mathrm{u}$

Cartesian coordinates: 


$\begin{array}{lrrr}\text { Pd } & 1.34897000 & 0.22862200 & -0.69322100 \\ \mathrm{C} & -0.21400900 & -0.91131300 & 0.22786900 \\ \mathrm{C} & 0.23075100 & -1.40315900 & 1.49727200 \\ \mathrm{C} & 0.34769900 & -1.54305200 & -0.94982300 \\ \mathrm{C} & 1.11667700 & -2.44309500 & 1.61187900 \\ \mathrm{C} & 1.24726200 & -2.64795000 & -0.79362900 \\ \mathrm{C} & 1.63794900 & -3.06428300 & 0.45291800 \\ \mathrm{C} & -1.52800100 & -0.21272600 & 0.17184100 \\ \mathrm{C} & -2.66149800 & -0.86483800 & -0.32150300 \\ \mathrm{C} & -1.69958200 & 1.09499900 & 0.63267200 \\ \mathrm{C} & -3.89857500 & -0.23324600 & -0.40321200 \\ \mathrm{C} & -2.92589900 & 1.74527500 & 0.56546900 \\ \mathrm{C} & -4.03107400 & 1.07729500 & 0.04478600 \\ \mathrm{~F} & -0.28305500 & -0.85069100 & 2.61106800 \\ \mathrm{~F} & -0.36609000 & -1.54488500 & -2.12076100 \\ \mathrm{~F} & 1.70219200 & -3.26725100 & -1.89349900 \\ \mathrm{~F} & 2.54210300 & -4.04403500 & 0.59812700 \\ \mathrm{~F} & 1.54457700 & -2.86000700 & 2.81473500 \\ \mathrm{~F} & -2.57749100 & -2.14095300 & -0.72326400 \\ \mathrm{~F} & -0.65775100 & 1.78161400 & 1.13414800 \\ \mathrm{~F} & -3.05011100 & 3.00613400 & 0.99768600 \\ \mathrm{~F} & -4.96197000 & -0.88373700 & -0.88954700 \\ \mathrm{~F} & -5.21481600 & 1.69229400 & -0.02101200 \\ \mathrm{O} & 2.44876400 & 2.12148900 & -0.66948300 \\ \mathrm{C} & 1.72608400 & 3.38938400 & -0.73748600 \\ \mathrm{C} & 3.65042500 & 2.27041800 & 0.13654900 \\ \mathrm{C} & 2.49314800 & 4.34428800 & 0.17759300 \\ \mathrm{H} & 1.73505400 & 3.71372800 & -1.78247300 \\ \mathrm{H} & 0.69891500 & 3.21044900 & -0.41723100 \\ \mathrm{C} & 3.91777500 & 3.76980800 & 0.15221000 \\ \mathrm{H} & 3.45025300 & 1.88156400 & 1.14130000 \\ \mathrm{H} & 4.43412200 & 1.67383800 & -0.33383900 \\ \mathrm{H} & 2.08792800 & 4.30485500 & 1.19379200 \\ \mathrm{H} & 2.43953300 & 5.37727800 & -0.17260400 \\ \mathrm{H} & 4.51714500 & 4.07281800 & 1.01352600 \\ \mathrm{H} & 4.44121400 & 4.07373800 & -0.75999400\end{array}$

18) $\left[\mathrm{Pd}\left(\mathrm{SMe}_{2}\right)_{2}\left(\mathrm{C}_{6} \mathrm{~F}_{5}\right)_{2}\right](\mathbf{1 2})$

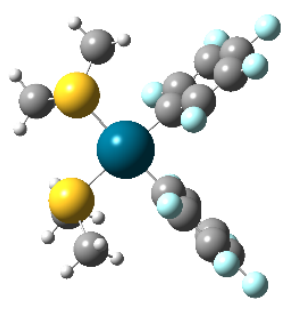

Number of imaginary frequencies $=0$

$E_{\text {total }}=-2539.68864396 \mathrm{a} . \mathrm{u}$

$\mathrm{G}_{\text {correction }}=0.189460 \mathrm{a} . \mathrm{u}$

\section{Cartesian coordinates:}

$\begin{array}{lrrr}\mathrm{Pd} & -0.03722900 & 1.16920400 & -0.27910000 \\ \mathrm{C} & 1.35261100 & -0.26964200 & 0.00536200 \\ \mathrm{C} & 1.67148000 & -0.76914200 & 1.25944000\end{array}$




\begin{tabular}{|c|c|c|c|}
\hline C & 2.06319400 & -0.80129000 & -1.06281100 \\
\hline $\mathrm{C}$ & 2.64314700 & -1.74638700 & 1.46273000 \\
\hline $\mathrm{C}$ & 3.04017900 & -1.78116900 & -0.90968600 \\
\hline $\mathrm{C}$ & 3.32864000 & -2.25964900 & 0.36563800 \\
\hline $\mathrm{C}$ & -1.38739200 & -0.32991400 & -0.1554550 \\
\hline $\mathrm{C}$ & -1.65957800 & -1.20205100 & -1.1995330 \\
\hline $\mathrm{C}$ & -2.10256100 & -0.53793700 & 1.0157680 \\
\hline $\mathrm{C}$ & -2.59466800 & -2.23019100 & -1.1020210 \\
\hline $\mathrm{C}$ & -3.04520000 & -1.55144100 & 1.1610350 \\
\hline $\mathrm{C}$ & -3.28898300 & -2.40780800 & 0.0911160 \\
\hline $\mathrm{F}$ & 1.04293700 & -0.28900400 & 2.3630480 \\
\hline $\mathrm{F}$ & 1.85330100 & -0.33611000 & -2.3199520 \\
\hline $\mathrm{F}$ & 3.71447500 & -2.26194700 & -1.9678850 \\
\hline $\mathrm{F}$ & 4.27050000 & -3.19967700 & 0.5369550 \\
\hline $\mathrm{F}$ & 2.92845700 & -2.19673600 & 2.6962770 \\
\hline $\mathrm{F}$ & -1.01939300 & -1.06561200 & -2.385698 \\
\hline $\mathrm{F}$ & -1.93484800 & 0.29841000 & 2.074798 \\
\hline $\mathrm{F}$ & -3.72575700 & -1.70928000 & 2.309350 \\
\hline $\mathrm{F}$ & -2.83620800 & -3.04929100 & -2.1395790 \\
\hline $\mathrm{F}$ & -4.19467900 & -3.39123400 & 0.205583 \\
\hline S & -1.75232200 & 2.80829200 & -0.79838600 \\
\hline S & 1.65192500 & 2.91970800 & -0.1873000 \\
\hline $\mathrm{C}$ & -1.58360000 & 4.15848000 & 0.4188500 \\
\hline $\mathrm{H}$ & -1.59808000 & 3.75838100 & 1.4338290 \\
\hline $\mathrm{H}$ & -0.63457000 & 4.65883100 & 0.2230220 \\
\hline $\mathrm{H}$ & -2.39984900 & 4.86883300 & 0.2774210 \\
\hline $\mathrm{C}$ & -3.38549900 & 2.16652500 & -0.3051740 \\
\hline $\mathrm{H}$ & -4.13351300 & 2.93926600 & -0.4885940 \\
\hline $\mathrm{H}$ & -3.59014500 & 1.29388300 & -0.9244720 \\
\hline $\mathrm{H}$ & -3.37271600 & 1.87793100 & 0.7455250 \\
\hline $\mathrm{C}$ & 3.26153600 & 2.25364500 & -0.7217850 \\
\hline $\mathrm{H}$ & 3.51597300 & 1.37596500 & -0.1279470 \\
\hline $\mathrm{H}$ & 3.16292000 & 1.97067700 & -1.76960600 \\
\hline $\mathrm{H}$ & 4.01797400 & 3.03184400 & -0.6106120 \\
\hline C & 1.93986500 & 3.03882200 & 1.6119530 \\
\hline $\mathrm{H}$ & 2.78719700 & 3.70119000 & 1.7970890 \\
\hline $\mathrm{H}$ & 1.03854700 & 3.46323500 & 2.0552000 \\
\hline $\mathrm{H}$ & 2.11704700 & 2.04781500 & 2.0300990 \\
\hline
\end{tabular}

\section{9) $\operatorname{TS}(\mathbf{1 2 - 1 3 )}$}

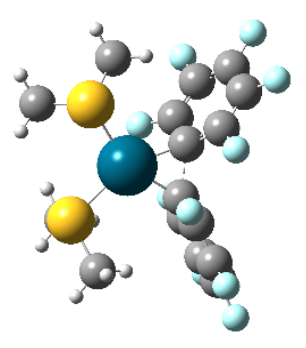

Number of imaginary frequencies $=1\left(i 121.48 \mathrm{~cm}^{-1}\right)$

$\mathrm{E}_{\text {total }}=-2539.63909494$ a.u

$\mathrm{G}_{\text {correction }}=0.188612 \mathrm{a} . \mathrm{u}$

\section{Cartesian coordinates:}


$-0.10418400$

0.89881000

1.51151100

1.71454400

2.75913200

2.96229600

3. 49353200

$-0.79049900$

$-1.28176500$

$-1.60260400$

$-2.42709100$

$-2.75026900$

$-3.17008000$

0.86417000

1. 35186600

3.69593200

4.69748500

3.25848500

$-0.61568000$

$-1.36849400$

$-3.50228200$

$-2.81650900$

$-4.27636100$

$-2.02213400$

1. 59113700

$-2.12767800$

$-2.11922100$

$-1.25007400$

$-3.03344800$

$-3.48986100$

$-4.39881800$

$-3.48812500$

$-3.42658300$

3. 20495300

3. 21268700

3. 33375300

4.00483700

1.54431200

2.35860500

0.58607800

1.61573900
1.24493700

$-0.57367200$

$-0.96156600$

$-0.81377300$

$-1.56347100$

$-1.41174400$

$-1.81051000$

$-0.73613900$

$-1.67389700$

$-0.62747700$

$-2.43395300$

$-1.37382700$

$-2.29947600$

$-0.77772500$

$-0.34032900$

$-1.55550600$

$-2.39191300$

$-1.91637100$

$-1.88845600$

0.33790800

$-1.16118600$

$-3.30920100$

$-3.02704500$

2.54022900

2.96748900

4.02727300

3.74392400

4.63666900

4.59010400

1.63491400

2.18129200

0.66422500

1.48865100

2.16162600

1. 18120200

2.04273500

2. 78681200

2. 92268700

3. 52560200

3. 34280100

1. 89185200
$-0.40035500$

$-0.00182400$

1.21429200

$-1.14284500$

1.29641800

$-1.08317000$

0.14106700

$-0.04959000$

$-0.98874200$

1. 11301900

$-0.79837400$

1. 32065800

0.36941500

2.38200800

$-2.35688200$

$-2.19793700$

0.20712300

2.49062300

$-2.13761000$

2.03462500

2.41249300

$-1.73743600$

0.56470400

$-1.16089300$

0.11482500

$-0.10618500$

0.94806000

$-0.32762500$

$-0.34070900$

$-0.56301100$

$-0.82179300$

$-1.06102900$

0.51653500

$-0.15354900$

0.32534900

$-1.23003500$

0.24783500

1. 94098600

2.34823600

2. 24927200

2. 29252000

20) $\left[\mathrm{Pd}\left(\mathrm{SMe}_{2}\right)_{2}\right]$ (13)

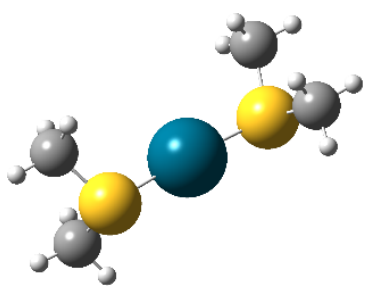

Number of imaginary frequencies $=0$

$E_{\text {total }}=-1084.04647072$ a.u

$\mathrm{G}_{\text {correction }}=0.114020 \mathrm{a} . \mathrm{u}$ 
Cartesian coordinates:

$\begin{array}{lrrr}\mathrm{Pd} & -0.00000700 & 0.17186500 & 0.00000700 \\ \mathrm{~S} & 2.26943100 & 0.25181700 & 0.43419800 \\ \mathrm{~S} & -2.26946800 & 0.25189300 & -0.43414700 \\ \mathrm{C} & 3.16451200 & 0.64285300 & -1.11482200 \\ \mathrm{H} & 2.89184500 & -0.06202900 & -1.90147300 \\ \mathrm{H} & 2.87034500 & 1.65071800 & -1.40931600 \\ \mathrm{H} & 4.24089000 & 0.61146900 & -0.92934000 \\ \mathrm{C} & 2.89190600 & -1.44997200 & 0.69722100 \\ \mathrm{H} & 3.97630600 & -1.42262500 & 0.82834900 \\ \mathrm{H} & 2.42035900 & -1.82765000 & 1.60504400 \\ \mathrm{H} & 2.62571100 & -2.08859700 & -0.14616600 \\ \mathrm{C} & -3.16465800 & 0.64261800 & 1.11488400 \\ \mathrm{H} & -2.89198700 & -0.06237800 & 1.90143300 \\ \mathrm{H} & -2.87058100 & 1.65045500 & 1.40957300 \\ \mathrm{H} & -4.24102400 & 0.61119400 & 0.92933600 \\ \mathrm{C} & -2.89166800 & -1.44996900 & -0.69739700 \\ \mathrm{H} & -3.97607700 & -1.42279500 & -0.82849600 \\ \mathrm{H} & -2.42007400 & -1.82744200 & -1.60528000 \\ \mathrm{H} & -2.62534700 & -2.08865900 & 0.14589900\end{array}$

21) $\left(\mathrm{C}_{6} \mathrm{~F}_{5}\right)_{2}$

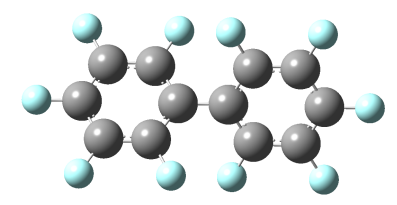

Number of imaginary frequencies $=0$

$E_{\text {total }}=-1455.61715120 \mathrm{a} . \mathrm{u}$

$\mathrm{G}_{\text {correction }}=0.053058 \mathrm{a} . \mathrm{u}$

\section{Cartesian coordinates:}

$$
\begin{aligned}
& \text { C } \\
& C \\
& C \\
& C \\
& C \\
& C \\
& C \\
& C \\
& C \\
& C \\
& C \\
& C \\
& C \\
& C \\
& C \\
& C \\
& \text { F } \\
& \text { F } \\
& \text { F } \\
& \text { F } \\
& \text { F } \\
& \text { F }
\end{aligned}
$$$$
\text { C }
$$$$
\text { C }
$$$$
\text { C }
$$$$
\text { C }
$$$$
\text { C }
$$$$
\text { F }
$$$$
\text { F }
$$$$
\text { F }
$$$$
\text { F }
$$$$
\text { F }
$$

$$
\begin{array}{r}
0.73801600 \\
1.46494300 \\
1.46496500 \\
2.85505300 \\
2.85508000 \\
3.55160500 \\
-0.73801800 \\
-1.46496500 \\
-1.46494300 \\
-2.85508400 \\
-2.85504900 \\
-3.55160300 \\
0.82200000 \\
0.82205800 \\
3.52371600 \\
4.88596800 \\
3.52367300 \\
-0.82206100 \\
-0.82199400 \\
-3.52368300
\end{array}
$$

-0.00000400
-0.53948300
0.53948600
-0.53916100
0.53916100
-0.00000500
-0.00000200
-0.53950000
0.53949100
-0.53917800
0.53916800
-0.00000100
-1.08571600
1.08578000
1.06415000
-0.00000100
-1.06412300
-1.08579700
1.08574200
1.06414500 


\section{2) $\left[\mathrm{Pd}\left(\mathrm{SMe}_{2}\right)\left(\mathrm{C}_{6} \mathrm{~F}_{5}\right)_{2}\right](\mathbf{1 4})$}

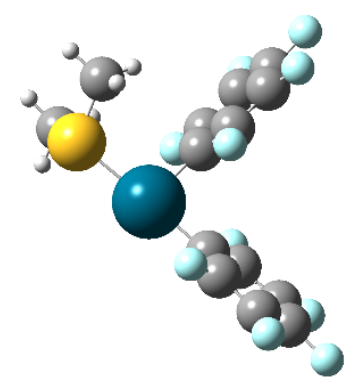

Number of imaginary frequencies $=0$

$E_{\text {total }}=-2061.60974416 \mathrm{a} . \mathrm{u}$

$\mathrm{G}_{\text {correction }}=0.117140 \mathrm{a} . \mathrm{u}$

\section{Cartesian coordinates:}

\begin{tabular}{|c|c|c|c|}
\hline $\mathrm{Pd}$ & -0.22117200 & -1.54748700 & -0.16254800 \\
\hline $\mathrm{C}$ & 1.55890200 & -0.60863200 & -0.24748600 \\
\hline C & 1.92650400 & 0.30835100 & -1.22553500 \\
\hline $\mathrm{C}$ & 2.51028500 & -0.88169200 & 0.72951300 \\
\hline $\mathrm{C}$ & 3.17762900 & 0.91946400 & -1.25042600 \\
\hline $\mathrm{C}$ & 3.77148400 & -0.29366100 & 0.74376000 \\
\hline C & 4.10395300 & 0.61588300 & -0.25657200 \\
\hline C & -1.02233400 & 0.23067600 & 0.11037400 \\
\hline C & -0.94782600 & 0.87824500 & 1.33702300 \\
\hline $\mathrm{C}$ & -1.73076100 & 0.86732800 & -0.90120400 \\
\hline C & -1.56783500 & 2.10420600 & 1.56626000 \\
\hline C & -2.36520700 & 2.09126200 & -0.70479100 \\
\hline C & -2.27990700 & 2.71404000 & 0.5374580 \\
\hline $\mathrm{F}$ & 1.06281300 & 0.64408200 & -2.2111710 \\
\hline $\mathrm{F}$ & 2.22865800 & -1.76104100 & 1.7234370 \\
\hline $\mathrm{F}$ & 4.66736900 & -0.58903500 & 1.6998830 \\
\hline $\mathrm{F}$ & 5.31425500 & 1.19123400 & -0.2672420 \\
\hline $\mathrm{F}$ & 3.50421900 & 1.79273000 & -2.2173210 \\
\hline $\mathrm{F}$ & -0.27494900 & 0.31940500 & 2.3661510 \\
\hline $\mathrm{F}$ & -1.85007400 & 0.29585700 & -2.1218580 \\
\hline $\mathrm{F}$ & -3.05415000 & 2.67446200 & -1.6971450 \\
\hline $\mathrm{F}$ & -1.49095300 & 2.69935200 & 2.7659660 \\
\hline $\mathrm{F}$ & -2.88666100 & 3.89046200 & 0.7440010 \\
\hline S & -2.31317200 & -2.75581900 & 0.0609510 \\
\hline C & -3.23303300 & -2.59018200 & -1.5068080 \\
\hline $\mathrm{H}$ & -4.22390300 & -3.03037500 & -1.3832150 \\
\hline $\mathrm{H}$ & -3.30177200 & -1.54327800 & -1.79917100 \\
\hline $\mathrm{H}$ & -2.67577500 & -3.14639600 & -2.2616020 \\
\hline C & -3.37983700 & -1.78828500 & 1.1793470 \\
\hline $\mathrm{H}$ & -3.50848600 & -0.77559100 & 0.7961490 \\
\hline $\mathrm{H}$ & -4.34261600 & -2.29225000 & 1.2745060 \\
\hline $\mathrm{H}$ & -2.87964500 & -1.75420600 & 2.14752600 \\
\hline
\end{tabular}

\section{3) $\mathrm{SMe}_{2}$}




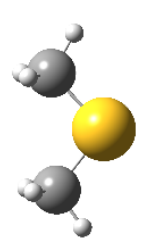

Number of imaginary frequencies $=0$

$\mathrm{E}_{\text {total }}=-478.03511113 \mathrm{a} . \mathrm{u}$

$\mathrm{G}_{\text {correction }}=0.049080 \mathrm{a} . \mathrm{u}$

\section{Cartesian coordinates:}

$\begin{array}{lrrr}\mathrm{C} & 1.39257100 & -0.51597100 & -0.00000900 \\ \mathrm{H} & 1.36890800 & -1.14417200 & 0.89380000 \\ \mathrm{H} & 1.36882000 & -1.14426000 & -0.89375300 \\ \mathrm{H} & 2.31391600 & 0.06945000 & -0.00008000 \\ \mathrm{C} & -1.39257100 & -0.51597100 & -0.00000400 \\ \mathrm{H} & -1.36885500 & -1.14422100 & -0.89377800 \\ \mathrm{H} & -1.36887400 & -1.14421200 & 0.89377600 \\ \mathrm{H} & -2.31391600 & 0.06945000 & -0.00001700 \\ \mathrm{~S} & 0.00000000 & 0.66435100 & 0.00000800\end{array}$

\section{4) TS(14-15)}

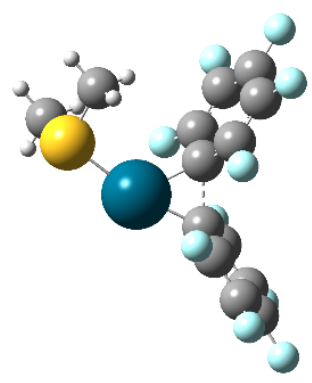

Number of imaginary frequencies $=1\left(i 250.02 \mathrm{~cm}^{-1}\right)$

$E_{\text {total }}=-2061.57323865 \mathrm{a} . \mathrm{u}$

$\mathrm{G}_{\text {correction }}=0.117148 \mathrm{a} . \mathrm{u}$

\section{Cartesian coordinates:}

$\begin{array}{lrrr}\mathrm{Pd} & 0.33257200 & -1.58779900 & 0.00994600 \\ \mathrm{C} & -1.19996500 & -0.19367400 & 0.11314700 \\ \mathrm{C} & -1.83802400 & 0.29095000 & 1.27318100 \\ \mathrm{C} & -2.07287000 & -0.46428000 & -0.96812500 \\ \mathrm{C} & -3.20408000 & 0.52658900 & 1.34953000 \\ \mathrm{C} & -3.44133500 & -0.23867000 & -0.91198600 \\ \mathrm{C} & -4.01544300 & 0.26868600 & 0.24956700 \\ \mathrm{C} & 0.47078000 & 0.46981100 & -0.09010500 \\ \mathrm{C} & 0.59784900 & 1.26110100 & -1.24893800 \\ \mathrm{C} & 1.27442200 & 0.89589800 & 0.99468200 \\ \mathrm{C} & 1.41470000 & 2.38248100 & -1.32368000 \\ \mathrm{C} & 2.10071200 & 2.00875900 & 0.93884100 \\ \mathrm{C} & 2.16696500 & 2.77324800 & -0.22240700 \\ \mathrm{~F} & -1.12188600 & 0.59488500 & 2.36808300 \\ \mathrm{~F} & -1.62223600 & -1.03840300 & -2.10315800 \\ \mathrm{~F} & -4.22365700 & -0.54772000 & -1.95599100\end{array}$


$-5.33213200$

$-3.74645100$

$-0.10864700$

1.36098400

2.87378700

1. 47359100

2.95869900

2. 37420800

3. 20625100

4.23371600

3.18458800

2. 65555400

3. 41107100

3. 38106500

4.43588400

2.98886200
0.48408700

1.00491200

0.96722300

0.15759300

2. 32958500

3.09611300

3. 85015600

$-2.85024400$

$-2.78163700$

$-3.13639400$

$-1.76468600$

$-3.44336600$

$-1.66417600$

$-0.68618800$

$-2.03650000$

$-1.58663200$
0.31550400

2. 47806500

$-2.35497800$

2. 12455700

1. 98741600

$-2.45766900$

$-0.28420700$

$-0.26860200$

1. 35831000

1.25581400

1.75135900

2.02810600

$-1.19453700$

$-0.71068900$

$-1.25100400$

$-2.19693200$

\section{5) Intermediate-15}

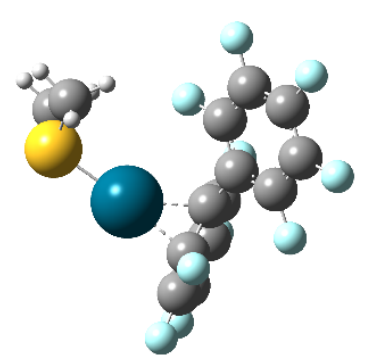

Number of imaginary frequencies $=0$

$\mathrm{E}_{\text {total }}=-2061.61611623 \mathrm{a} . \mathrm{u}$

$\mathrm{G}_{\text {correction }}=0.118200 \mathrm{a} . \mathrm{u}$

\section{Cartesian coordinates:}

$\mathrm{Pd}$
$C$
$C$
$C$
$C$
$C$
$C$
$C$
$C$
$C$
$C$
$C$
$C$
$C$
$C$
$C$
F
F
F
F
F
F
F
F

$$
\begin{array}{r}
1.22787300 \\
0.22723900 \\
0.85547100 \\
0.97909200 \\
2.10538400 \\
2.26280100 \\
2.82038600 \\
-1.24824300 \\
-2.07542600 \\
-1.87566400 \\
-3.45679800 \\
-3.25258100 \\
-4.04793300 \\
0.18147200 \\
0.33467300 \\
2.91355700 \\
4.05432200 \\
2.68224000 \\
-1.54417100 \\
-1.14837200 \\
-3.81560600
\end{array}
$$

0.91515600

$-0.81892900$

$-1.06992600$

$-1.16779400$

$-1.63647700$

$-1.77187500$

$-1.97935900$

$-0.64121400$

$-1.62183600$

0.48605800

$-1.46778200$

0.66069800

$-0.32215800$

$-0.77809100$

$-1.38003000$

$-2.14274500$

$-2.48508200$

$-1.82698700$

$-2.75600600$

1.46439600

1.76515600
$-0.66754500$

0.23308100

1.48899200

$-0.94414600$

1.58624600

$-0.82004400$

0.42128400

0.19281900

$-0.36260700$

0.73155100

$-0.43144200$

0.67574200

0.09187900

2. 61369200

$-2.12567800$

$-1.93021300$

0.53993500

2.78166400

$-0.83879600$

1.29839700

1.17965600 


$\begin{array}{lr}\text { F } & -4.21784600 \\ \text { F } & -5.37268300 \\ \text { S } & 1.47815200 \\ \text { C } & 1.80687100 \\ \text { H } & 1.79290200 \\ H & 1.06256200 \\ H & 2.79790300 \\ \text { C } & -0.22424200 \\ H & -0.88082000 \\ H & -0.21437100 \\ H & -0.55719700\end{array}$

-2.42273600
-0.16636700
3.28551400
3.77484600
4.86396700
3.33234600
3.39945800
3.93166500
3.46563200
5.01696400
3.67713600

$-0.97695700$

0.03815500

$-0.72008600$

1.01115700

1.08885400

1.67428200

1.26834700

$-0.90199800$

$-0.16709200$

$-0.78084900$

$-1.90886100$

\section{6) $\left[\mathrm{Pd}\left(\mathrm{PMe}_{3}\right)_{2}\left(\mathrm{C}_{6} \mathrm{~F}_{5}\right)_{2}\right]$ (16)}

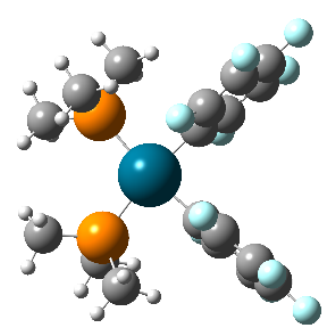

Number of imaginary frequencies $=0$

$\mathrm{E}_{\text {total }}=-2505.92212439$ a.u

$\mathrm{G}_{\text {correction }}=0.260071 \mathrm{a} . \mathrm{u}$

\section{Cartesian coordinates:}

$\begin{array}{lr}\mathrm{Pd} & 0.00012100 \\ \mathrm{C} & 1.39492700 \\ \mathrm{C} & 2.18768300 \\ \mathrm{C} & 1.59409300 \\ \mathrm{C} & 3.13778800 \\ \mathrm{C} & 2.52983700 \\ \mathrm{C} & 3.30608600 \\ \mathrm{C} & -1.39482600 \\ \mathrm{C} & -2.18752500 \\ \mathrm{C} & -1.59406800 \\ \mathrm{C} & -3.13766100 \\ \mathrm{C} & -2.52982800 \\ \mathrm{C} & -3.30604900 \\ \mathrm{~F} & 2.08286300 \\ \mathrm{~F} & 0.87556500 \\ \mathrm{~F} & 2.70024500 \\ \mathrm{~F} & 4.21528800 \\ \mathrm{~F} & 3.89306300 \\ \mathrm{~F} & -2.08251600 \\ \mathrm{~F} & -0.87557900 \\ \mathrm{~F} & -2.70029400 \\ \mathrm{~F} & -3.89284600 \\ \mathrm{~F} & -4.21526200 \\ \mathrm{C} & -3.25821400 \\ \mathrm{H} & -3.66726700 \\ \mathrm{H} & -2.99294900 \\ \mathrm{H} & -4.00836300 \\ & \end{array}$

1.00214300

$-0.50641200$

$-0.81099700$

$-1.28397600$

$-1.82881600$

$-2.31410600$

$-2.58878300$

$-0.50635900$

$-0.81119700$

$-1.28369800$

$-1.82898900$

$-2.31379600$

$-2.58869200$

$-0.07424900$

$-1.04019600$

$-3.03974700$

$-3.57544400$

$-2.08535800$

$-0.07485100$

$-1.03969500$

$-3.03919600$

$-2.08576700$

$-3.57533300$

1.86797300

1.06664500

1.44632800

2. 65293200
0.00005100

$-0.08773600$

1.00794700

$-1.21706800$

1.00247900

$-1.27529900$

$-0.15235200$

0.08761000

$-1.00803100$

1.21709800

$-1.00238400$

1.27552100

0.15260200

2.14809600

$-2.34246300$

$-2.39371000$

$-0.18428700$

2.08441900

$-2.14842900$

2. 34248000

2.39407700

$-2.08433100$

0.18469600

1.05342000

0.43666500

2.02566000

1.18507700 
$-2.37292500$

$-3.20011800$

$-1.56428800$

$-2.70946200$

3.25871000

3.66796100

2.99390000

4.00854500

2.37211600

3.19886900

1.56309800

2. 70922800

1.74355300

$-1.74363600$

$-1.40530400$

$-0.59079700$

$-2.29541000$

$-1.11043100$

1.40437200

0.58885200

2.29379400

1.11052700
3.26008100

3. 95322500

3. 78285700

2. 43949400

1.86841700

1.06766300

1.44600000

2.65362000

3. 26231600

3. 95582500

3.78506300

2. 44263700

2.54515500

2.54490300

4.01189400

4.60998100

4.64165800

3.66659300

4.01085800

4.60828700

4.64159500

3.66434100
$-1.30967500$

$-1.13045500$

$-1.82463700$

$-1.94699200$

$-1.05242400$

$-0.43505000$

$-2.02445100$

$-1.18439500$

1.30908400

1.12924900

1.82346000

1.94726400

$-0.26220600$

0.26223800

1. 32472700

0.91280500

1.41428100

2.31909100

$-1.32617100$

$-0.91530300$

$-1.41570000$

$-2.32041200$

\section{7) $\operatorname{TS}(16-17)$}

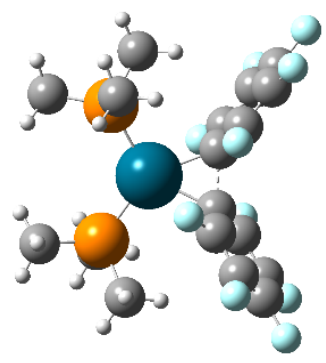

Number of imaginary frequencies $=1\left(i 166.00 \mathrm{~cm}^{-1}\right)$

$\mathrm{E}_{\text {total }}=-2505.86659944 \mathrm{a} . \mathrm{u}$

$\mathrm{G}_{\text {correction }}=0.258635 \mathrm{a} . \mathrm{u}$

\section{Cartesian coordinates:}

$\mathrm{Pd}$
$\mathrm{C}$
$\mathrm{C}$
$\mathrm{C}$
$\mathrm{C}$
$\mathrm{C}$
$\mathrm{C}$
$\mathrm{C}$
$\mathrm{C}$
$\mathrm{C}$
$\mathrm{C}$
$\mathrm{C}$
$\mathrm{C}$
$\mathrm{F}$
$\mathrm{F}$

$$
\begin{array}{r}
-0.04586900 \\
0.88592100 \\
1.55550300 \\
1.58285600 \\
2.75265000 \\
2.77742400 \\
3.37065100 \\
-0.82782100 \\
-1.43241300 \\
-1.52451400 \\
-2.57687900 \\
-2.66973500 \\
-3.20275500 \\
1.03132700 \\
1.16991700
\end{array}
$$

1.15699600

$-0.77733200$

$-1.39453000$

$-0.89115600$

$-2.08406900$

$-1.57518800$

$-2.19695500$

$-0.84890300$

$-1.61825100$

$-0.90369500$

$-2.38162900$

$-1.65445300$

$-2.41824600$

$-1.32399700$

$-0.18814700$
$-0.08477800$

$-0.04180800$

1.03966200

$-1.27475100$

0.91716700

$-1.42204400$

$-0.32518900$

0.01896400

$-1.00053700$

1.25563300

$-0.81920100$

1. 45886900

0.42316500

2.27956800

$-2.35476000$ 


$\begin{array}{lrrr}\text { F } & 3.40337600 & -1.59327000 & -2.60923100 \\ F & 4.51764800 & -2.87477000 & -0.46025100 \\ F & 3.31884500 & -2.64761000 & 1.99548400 \\ F & -0.89311900 & -1.63958600 & -2.23388300 \\ F & -1.17601300 & -0.08198900 & 2.27347000 \\ F & -3.30876800 & -1.60427000 & 2.63837300 \\ F & -3.08212200 & -3.09065200 & -1.84026600 \\ F & -4.30176600 & -3.15860800 & 0.61474300 \\ \mathrm{C} & -3.55180800 & 1.55409500 & -0.14110000 \\ \mathrm{H} & -3.57606000 & 0.54388200 & -0.55777200 \\ \mathrm{H} & -3.58605000 & 1.47214700 & 0.94834800 \\ \mathrm{H} & -4.42722400 & 2.11061600 & -0.49017000 \\ \mathrm{C} & -2.21695900 & 2.56936500 & -2.46465400 \\ \mathrm{H} & -3.15445700 & 3.08738300 & -2.69177100 \\ \mathrm{H} & -1.38021400 & 3.13505200 & -2.88226900 \\ \mathrm{H} & -2.22547200 & 1.58181600 & -2.93307700 \\ \mathrm{C} & 3.42873700 & 1.86040200 & -0.04749900 \\ \mathrm{H} & 3.53313600 & 0.79745200 & 0.18289100 \\ \mathrm{H} & 3.46558000 & 1.97840800 & -1.13356700 \\ \mathrm{H} & 4.25926900 & 2.41064300 & 0.40577600 \\ \mathrm{C} & 2.03635000 & 2.29727900 & 2.41228800 \\ \mathrm{H} & 2.94524100 & 2.81098600 & 2.74262100 \\ \mathrm{H} & 1.17213100 & 2.72330100 & 2.92806700 \\ \mathrm{H} & 2.09818500 & 1.23889800 & 2.67746900 \\ \mathrm{P} & 1.79585100 & 2.44258200 & 0.58691700 \\ \mathrm{P} & -1.97273500 & 2.36623200 & -0.64662200 \\ \mathrm{C} & -2.20087600 & 4.08865000 & -0.02476500 \\ \mathrm{H} & -1.36861200 & 4.70597400 & -0.37329000 \\ \mathrm{H} & -3.14343700 & 4.52765400 & -0.36779300 \\ \mathrm{H} & -2.18546200 & 4.07909900 & 1.06847000 \\ \mathrm{C} & 1.89783200 & 4.26718900 & 0.32608800 \\ \mathrm{H} & 1.01105600 & 4.73936000 & 0.75742900 \\ \mathrm{H} & 2.79346700 & 4.69380700 & 0.78933100 \\ \mathrm{H} & 1.91323200 & 4.48015000 & -0.74623000 \\ & & & \end{array}$

\section{8) $\left[\mathrm{Pd}\left(\mathrm{PMe}_{3}\right)_{2}\right](\mathbf{1 7})$}

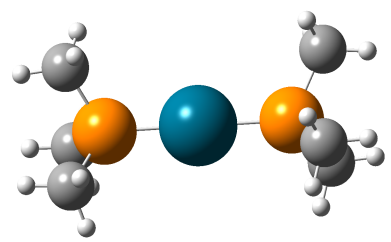

Number of imaginary frequencies $=0$

$E_{\text {total }}=-1050.27777395$ a.u

$\mathrm{G}_{\text {correction }}=0.182638 \mathrm{a} . \mathrm{u}$

\section{Cartesian coordinates:}

$\begin{array}{lrrr}\mathrm{Pd} & -0.00001000 & 0.00151700 & 0.00019300 \\ \mathrm{C} & 3.13637900 & -0.47475400 & 1.57917000 \\ \mathrm{H} & 2.82601600 & 0.20986600 & 2.37250300 \\ \mathrm{H} & 2.82833700 & -1.48468200 & 1.86157600 \\ \mathrm{H} & 4.22698100 & -0.44327200 & 1.47939700 \\ \mathrm{C} & 3.13706100 & 1.60400700 & -0.38007300\end{array}$


29) $\left(\mathrm{C}_{6} \mathrm{~F}_{5}\right)_{2}$

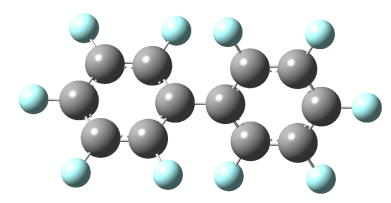

4.22758400

2.82759500

2. 82862500

$-3.13681200$

$-2.82981500$

$-2.82620100$

$-4.22735200$

$-3.13568100$

$-4.22623300$

$-2.82446600$

$-2.82825600$

$-2.29756100$

2. 29773400

3.13649600

2.82664100

4.22698400

2. 82857100

$-3.13759200$

$-2.82778600$

$-4.22804900$

$-2.83039600$
1.50166700

1. 94736400

2. 35448500

1.59123900

1.90194500

2. 36543300

1.49029700

$-1.16852500$

$-1.09613200$

$-2.19089100$

$-0.94087000$

$-0.00054500$

$-0.00041800$

$-1.13217800$

$-0.87489200$

$-1.05940800$

$-2.16170900$

$-0.42559600$

$-1.42501100$

$-0.39985700$

0.28518800
$-0.35629700$

$-1.37037800$

0.35209500

0.42947100

1.43137200

$-0.27664900$

0.39976700

1.16407200

1.08878700

0.93495400

2.18782700

$-0.00039900$

$-0.00027000$

$-1.19954200$

$-2.21559700$

$-1.12378300$

$-1.00045300$

$-1.59315500$

$-1.90878500$

$-1.49046800$

$-2.36437900$

Number of imaginary frequencies $=0$

$\mathrm{E}_{\text {total }}=-1455.61597898 \mathrm{a} . \mathrm{u}$

$\mathrm{G}_{\text {correction }}=0.053206 \mathrm{a} . \mathrm{u}$

\section{Cartesian coordinates:}

C
C
C
C
C
C
C
C
C
C
C
C
F
F
F
F
F
F
F
0.73804500

1.46466400

1.46467200

2.85505900

2.85507100

3.55159100

$-0.73804600$

$-1.46467100$

$-1.46466500$

$-2.85507400$

$-2.85505600$

$-3.55158800$

0.82124900

0.82127400

3.52354200

4.88545600

3.52352900

$-0.82127600$

$-0.82124400$
0.00001000

$-1.06542100$

1.06543100

$-1.07802400$

1.07802100

$-0.00000200$

0.00001000

1.06542200

$-1.06542000$

1.07801700

$-1.07801900$

0.00000100

$-2.10397800$

2.10398700

2.10938900

$-0.00001400$

$-2.10939800$

2.10397800

$-2.10396400$
0.00000000

$-0.54029900$

0.54029800

$-0.53995000$

0.53995000

$-0.00000100$

$-0.00000200$

$-0.54031000$

0.54031000

$-0.53996500$

0.53995900

$-0.00000300$

$-1.08726300$

1.08728100

1.06534900

$-0.00000100$

$-1.06533800$

$-1.08730000$

1.08728700 


$\begin{array}{lrrr}F & -3.52353800 & -2.10938000 & 1.06535800 \\ F & -3.52353600 & 2.10938500 & -1.06537100 \\ F & -4.88545500 & -0.00002200 & 0.00000500\end{array}$

\section{0) $\left[\mathrm{Pd}\left(\mathrm{PMe}_{3}\right)\left(\mathrm{C}_{6} \mathrm{~F}_{5}\right)_{2}\right]$ (18)}

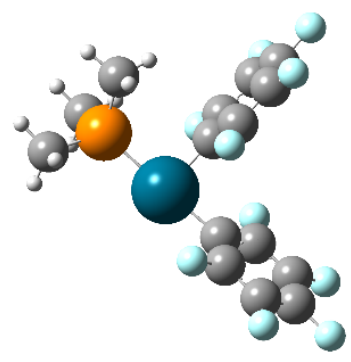

Number of imaginary frequencies $=0$

$E_{\text {total }}=-2044.71825905 \mathrm{a} . \mathrm{u}$

$\mathrm{G}_{\text {correction }}=0.152695 \mathrm{a} . \mathrm{u}$

\section{Cartesian coordinates:}

$\begin{array}{lrrr}\text { Pd } & -0.29890000 & -1.37438700 & -0.25690900 \\ \text { C } & 1.60432200 & -0.63535900 & -0.29651300 \\ \text { C } & 2.07977900 & 0.31819700 & -1.18820500 \\ \text { C } & 2.51410500 & -1.10183300 & 0.64469100 \\ \text { C } & 3.39247500 & 0.78313500 & -1.16468200 \\ \text { C } & 3.83473200 & -0.66906700 & 0.70819100 \\ \text { C } & 4.27296800 & 0.28495100 & -0.20723400 \\ \text { C } & -0.92243500 & 0.46569000 & 0.09801200 \\ \text { C } & -0.77921900 & 1.03944900 & 1.35431800 \\ \text { C } & -1.54901000 & 1.22220900 & -0.88383100 \\ \text { C } & -1.24863200 & 2.31990800 & 1.63826800 \\ \text { C } & -2.03117200 & 2.50433800 & -0.63181400 \\ \text { C } & -1.87654700 & 3.05657000 & 0.63736700 \\ \text { F } & 1.26455400 & 0.83790900 & -2.13523900 \\ \text { F } & 2.12369900 & -2.03463600 & 1.55280700 \\ \text { F } & 4.68701000 & -1.15217200 & 1.62668700 \\ \text { F } & 5.54113400 & 0.71564800 & -0.17247800 \\ \text { F } & 3.82290000 & 1.69791600 & -2.04866800 \\ \text { F } & -0.19079200 & 0.35239400 & 2.35606700 \\ \text { F } & -1.74118400 & 0.71516000 & -2.12274800 \\ \text { F } & -2.64232200 & 3.20906000 & -1.59487500 \\ \text { F } & -1.10787300 & 2.84607800 & 2.86329600 \\ \text { F } & -2.33672500 & 4.28729300 & 0.89580800 \\ \text { P } & -2.52478000 & -2.15059500 & -0.12122100 \\ \text { C } & -3.49166100 & -2.06672200 & -1.68124800 \\ \text { H } & -3.01199600 & -2.69306600 & -2.43761200 \\ \text { H } & -4.51920500 & -2.40843000 & -1.52558100 \\ \text { H } & -3.49342100 & -1.03688200 & -2.04216100 \\ \text { C } & -3.56313500 & -1.23676200 & 1.08652100 \\ \text { H } & -4.56565400 & -1.66941800 & 1.14635000 \\ \text { H } & -3.08835100 & -1.27330600 & 2.06979100 \\ \text { H } & -3.63529000 & -0.19132300 & 0.77980700 \\ \text { C } & -2.68798400 & -3.90618500 & 0.40606300 \\ & & & \end{array}$




$\begin{array}{lrrr}\mathrm{H} & -2.22789600 & -4.03574400 & 1.38885400 \\ \mathrm{H} & -3.74042500 & -4.20055900 & 0.45901300 \\ \mathrm{H} & -2.17176000 & -4.55352700 & -0.30768100\end{array}$

31) $\mathrm{PMe}_{3}$

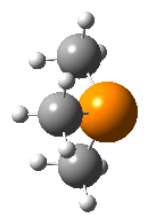

Number of imaginary frequencies $=0$

$\mathrm{E}_{\text {total }}=-461.13081969 \mathrm{a} . \mathrm{u}$

$\mathrm{G}_{\text {correction }}=0.083723 \mathrm{a} . \mathrm{u}$

Cartesian coordinates:

$\begin{array}{lrrr}\mathrm{C} & 0.64436700 & -1.50598000 & 0.28050100 \\ \mathrm{H} & 0.05787200 & -2.38203400 & -0.01112000 \\ \mathrm{H} & 1.68338900 & -1.68592100 & -0.01030800 \\ \mathrm{H} & 0.59635600 & -1.39501800 & 1.36957900 \\ \mathrm{C} & -1.62648300 & 0.19514200 & 0.28062200 \\ \mathrm{H} & -1.50697000 & 0.17920200 & 1.36974100 \\ \mathrm{H} & -2.09097800 & 1.14198700 & -0.00962200 \\ \mathrm{H} & -2.30248300 & -0.61356200 & -0.01195100 \\ \mathrm{P} & -0.00013900 & -0.00004200 & -0.60624700 \\ \mathrm{C} & 0.98234600 & 1.31090800 & 0.28043800 \\ \mathrm{H} & 0.61828600 & 2.30078600 & -0.00965100 \\ \mathrm{H} & 0.91107300 & 1.21333500 & 1.36951200 \\ \mathrm{H} & 2.03415400 & 1.24143400 & -0.01184400\end{array}$

32) $\operatorname{TS}(\mathbf{1 8 - 1 9 )}$

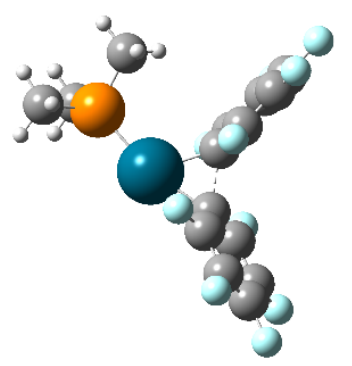

Number of imaginary frequencies $=1\left(i 279.53 \mathrm{~cm}^{-1}\right)$

$\mathrm{E}_{\text {total }}=-2044.68525615 \mathrm{a} . \mathrm{u}$

$\mathrm{G}_{\text {correction }}=0.152850 \mathrm{a} . \mathrm{u}$

\section{Cartesian coordinates:}

$\begin{array}{lrrr}\mathrm{Pd} & -0.49495600 & -1.38282900 & -0.19761700 \\ \mathrm{C} & 1.28248000 & -0.28077700 & -0.14902400 \\ \mathrm{C} & 2.02717600 & 0.21029800 & -1.23721700 \\ \mathrm{C} & 2.05450700 & -0.79223200 & 0.91796300 \\ \mathrm{C} & 3.41607000 & 0.23179100 & -1.25866500 \\ \mathrm{C} & 3.44348900 & -0.78325500 & 0.91744800\end{array}$


4.13313700

$-0.30068700$

$-0.32346000$

$-0.95232600$

$-0.91519300$

$-1.55346200$

$-1.52742000$

1.40879800

1.47284200

4.12700900

5.46832600

4.06977300

0.25235200

$-1.12627400$

$-2.19840500$

$-0.89160000$

$-2.09840000$

$-2.71079700$

$-3.59036300$

$-3.12935400$

$-4.65138700$

$-3.49150400$

$-3.73736100$

$-4.79546800$

$-3.37593300$

$-3.62079700$

$-3.15205900$

$-2.77598300$

$-4.23611300$

$-2.68050200$
$-0.26015100$

0.65375900

1.32973900

1. 32915100

2.57524300

2.57067800

3. 21246700

0.72689500

$-1.39416300$

$-1.31187500$

$-0.25087600$

0.72530800

0.78448000

0.73138300

3.14003300

3.16883200

4. 41247300

$-2.10445200$

$-2.36585400$

$-3.19825200$

$-2.58273100$

$-1.46621400$

$-0.80677400$

$-1.08501200$

$-0.65335100$

0.13314800

$-3.61991200$

$-3.52678800$

$-3.76992200$

$-4.49139500$
$-0.17241500$

0.09074700

1.32355900

$-0.96474600$

1.49714400

$-0.81141000$

0.42380800

$-2.31032300$

1. 97536200

1. 94135900

$-0.18518300$

$-2.31863700$

2. 40951900

$-2.16277100$

$-1.84049700$

2.69900500

0.57949100

$-0.07300500$

$-1.67204500$

$-2.20988200$

$-1.51249100$

$-2.28444600$

0.73999200

0.76547100

1. 75986300

0.19452700

0.88100200

1. 90292000

0.90733600

0.41938800

\section{3) Intermediate-19}

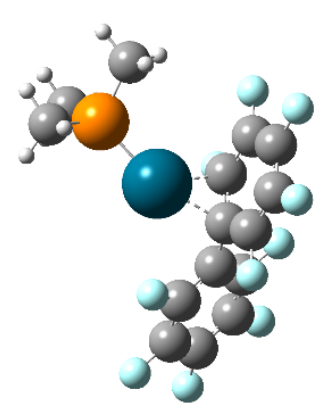

Number of imaginary frequencies $=0$

$\mathrm{E}_{\text {total }}=-2044.73094521 \mathrm{a} . \mathrm{u}$

$\mathrm{G}_{\text {correction }}=0.152703 \mathrm{a} . \mathrm{u}$

\section{Cartesian coordinates:}

$\begin{array}{lr}\mathrm{Pd} & 1.13905800 \\ \mathrm{C} & -0.40652200 \\ \mathrm{C} & -0.12529500 \\ \mathrm{C} & 0.49998000 \\ \mathrm{C} & 0.92293800 \\ \mathrm{C} & 1.54159900 \\ \mathrm{C} & 1.76190500\end{array}$

-0.73809000
0.86291300
1.76486200
0.88246500
2.65541100
1.84937800
2.69593200

$-0.31932000$

$-0.16852700$

$-1.23677400$

0.94801300

$-1.19182500$

1.00180100

$-0.05807300$ 
$-1.76095200$

$-2.64597600$

$-2.21557400$

$-3.91786900$

$-3.48451900$

$-4.33869300$

$-0.94665000$

0.09711200

2. 34835200

2.80711800

1.18115400

$-2.28097200$

$-1.40733100$

$-3.88492000$

$-4.74178000$

$-5.55898200$

2.97618200

3.76553500

4.05208300

4.65324700

3.04616700

2. 76627100

3.70968600

2.40870200

2.01726200

4.39389900

4.05986600

5.23631900

4.71898400
0.26592100

0.68834400

$-0.71845700$

0.13986700

$-1.27518100$

$-0.84353000$

1.77460400

0.37752200

1. 87179700

3. 53184400

3.47294600

1.65644800

$-1.17256000$

$-2.22405900$

0.56286800

$-1.37212200$

$-2.01602200$

$-3.10055800$

$-2.49792300$

$-3.60088000$

$-3.85457000$

$-3.17595300$

$-3.67227500$

$-2.61685800$

$-3.93027700$

$-0.97430000$

$-0.32749000$

$-1.58665000$

$-0.33692000$
$-0.04951000$

0.94633000

$-0.92995600$

1.07948500

$-0.82375100$

0.18843800

$-2.29811100$

2. 14475700

2.07396800

$-0.05581900$

$-2.22333200$

1.79702800

$-1.90350500$

$-1.67694600$

2.04409000

0.30310100

0.18616200

$-1.08245300$

$-1.94807000$

$-0.68174500$

$-1.41233700$

1.60539400

1.85579900

2.47353300

1.35175000

0.74577000

1.56077000

1.08398000

$-0.08063400$

\section{4) $\left[\mathrm{Pd}\left(\mathrm{C}_{6} \mathrm{H}_{6}\right)\left(\mathrm{C}_{6} \mathrm{~F}_{5}\right)_{2}\right]$ (20)}

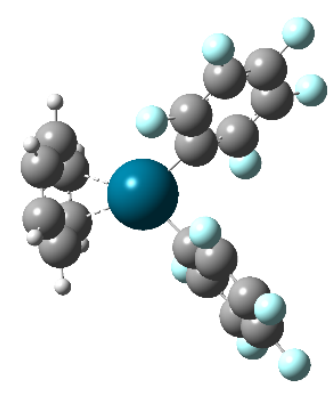

Number of imaginary frequencies $=0$

$\mathrm{E}_{\text {total }}=-1815.83598343 \mathrm{a} \cdot \mathrm{u}$

$\mathrm{G}_{\text {correction }}=0.139543 \mathrm{a} . \mathrm{u}$

\section{Cartesian coordinates:}

$\mathrm{Pd}$
$\mathrm{C}$
$\mathrm{C}$
$\mathrm{C}$
$\mathrm{C}$
$\mathrm{C}$
$\mathrm{C}$
$\mathrm{C}$

0.00015600

1.33792000

2.36542600

1.33360800

3.36174000

2.31389200

3.33181400

$-1.33803100$
1.35136400

$-0.09891300$

$-0.21497200$

$-1.00672000$

$-1.18078600$

$-1.98746800$

$-2.07448500$

$-0.09842300$
$-0.00064100$

$-0.21526400$

0.71410700

$-1.26716000$

0.60880600

$-1.40437600$

$-0.45879600$

0.21488000 


\begin{tabular}{|c|c|c|c|}
\hline C & -2.36548600 & -0.21483700 & -0.71449600 \\
\hline C & -1.33401700 & -1.00553400 & 1.26737800 \\
\hline C & -3.36204100 & -1.18034600 & -0.60863900 \\
\hline C & -2.31455900 & -1.98593800 & 1.40517800 \\
\hline C & -3.33242000 & -2.07333400 & 0.45956500 \\
\hline $\mathrm{F}$ & 2.42858300 & 0.63187700 & 1.76841800 \\
\hline $\mathrm{F}$ & 0.36851300 & -0.96363100 & -2.21014400 \\
\hline $\mathrm{F}$ & 2.29087800 & -2.84408300 & -2.43666100 \\
\hline $\mathrm{F}$ & 4.28452500 & -3.00816800 & -0.57991200 \\
\hline $\mathrm{F}$ & 4.34445200 & -1.26232700 & 1.51890200 \\
\hline F & -2.42832800 & 0.63131900 & -1.76937900 \\
\hline $\mathrm{F}$ & -0.36897500 & -0.96207100 & 2.21040100 \\
\hline $\mathrm{F}$ & -2.29184400 & -2.84186900 & 2.43803600 \\
\hline $\mathrm{F}$ & -4.34470100 & -1.26226900 & -1.51875800 \\
\hline $\mathrm{F}$ & -4.28537500 & -3.00669600 & 0.58123900 \\
\hline C & 0.10531100 & 3.61160100 & -1.40979600 \\
\hline C & 1.26103100 & 3.56308200 & -0.60827900 \\
\hline C & 1.15792600 & 3.58606300 & 0.7993990 \\
\hline C & -0.10400900 & 3.61222900 & 1.40992700 \\
\hline C & -1.25978500 & 3.56394800 & 0.60843100 \\
\hline C & -1.15672300 & 3.58630900 & -0.79924200 \\
\hline $\mathrm{H}$ & 0.19055200 & 3.61487800 & -2.49017500 \\
\hline $\mathrm{H}$ & 2.24028100 & 3.52942800 & -1.0728650 \\
\hline $\mathrm{H}$ & 2.05596400 & 3.56381700 & 1.405432 \\
\hline $\mathrm{H}$ & -0.18927700 & 3.61604300 & 2.4903020 \\
\hline $\mathrm{H}$ & -2.23904200 & 3.53105800 & 1.0730540 \\
\hline $\mathrm{H}$ & -2.05476600 & 3.56442000 & \\
\hline
\end{tabular}

\section{5) $\mathbf{T S}(\mathbf{2 0 - 2 1})$}

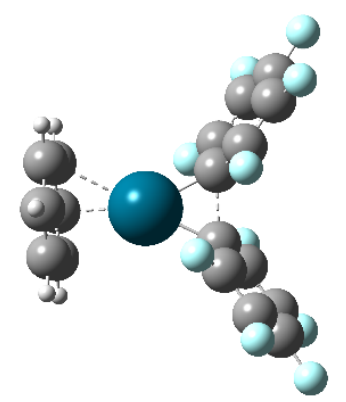

Number of imaginary frequencies $=1\left(i 264.41 \mathrm{~cm}^{-1}\right)$

$\mathrm{E}_{\text {total }}=-1815.80674228 \mathrm{a} . \mathrm{u}$

$\mathrm{G}_{\text {correction }}=0.140908 \mathrm{a} . \mathrm{u}$

\section{Cartesian coordinates:}

$\begin{array}{lrrr}\mathrm{Pd} & -0.44568900 & 1.24318500 & -0.51061500 \\ \mathrm{C} & -0.64003900 & -0.78018200 & -0.17269500 \\ \mathrm{C} & -0.62728400 & -1.81557500 & -1.12500800 \\ \mathrm{C} & -1.56088900 & -0.95384800 & 0.88457700 \\ \mathrm{C} & -1.43062600 & -2.94507400 & -1.02659700 \\ \mathrm{C} & -2.37416700 & -2.07355200 & 0.99907600 \\ \mathrm{C} & -2.30562200 & -3.08471000 & 0.04545700 \\ \mathrm{C} & 1.05198400 & -0.05389000 & 0.05520400 \\ \mathrm{C} & 1.53414400 & -0.28002700 & 1.35708500\end{array}$


2.03871300

2. 87239200

3.38049600

3.80479700

0.20767600

$-1.75916900$

$-3.25592900$

$-3.08474900$

$-1.36109100$

0.69169400

1. 73205200

4.28039700

3.26725300

5.09457900

$-2.86140700$

$-2.45827200$

$-1.16717200$

$-0.27800600$

$-0.69980200$

$-1.99195500$

$-3.85886600$

$-3.15275700$

$-0.89797700$

0.70704200

$-0.01816300$

$-2.31572700$
$-0.05630500$

$-0.52908700$

$-0.30203600$

$-0.55033700$

$-1.76809300$

0.01452600

$-2.16925400$

$-4.16530100$

$-3.90487800$

$-0.30178600$

0.26508800

$-0.25961100$

$-0.75545200$

$-0.78464600$

3.17522800

2.83651800

3.19645100

3.84699200

4.17660600

3.85775900

2. 91185400

2.35386300

3.06327700

4.14176400

4.69113900

4.12666700
$-0.95549900$

1.63687500

$-0.69691100$

0.60529200

$-2.17515100$

1.80104000

2.00374500

0.14721000

$-1.95866600$

2.40206400

$-2.22823400$

$-1.68881500$

2.89698300

0.86295700

0.32846800

$-0.97106600$

$-1.42608400$

$-0.53770800$

0.75822900

1.18270500

0.66479200

$-1.65026900$

$-2.46965200$

$-0.88349000$

1.42769700

2.18262400

\section{6) Intermediate-21}

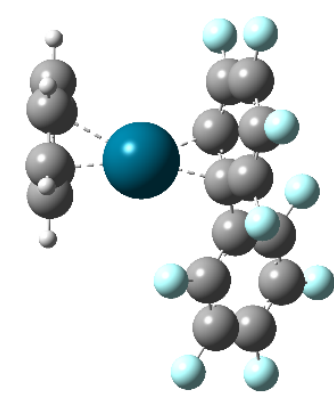

Number of imaginary frequencies $=0$

$$
\mathrm{E}_{\text {total }}=-1815.85117254 \mathrm{a} . \mathrm{u}
$$

$\mathrm{G}_{\text {correction }}=0.140755 \mathrm{a} . \mathrm{u}$

\section{Cartesian coordinates:}

$\mathrm{Pd}$
$\mathrm{C}$
$\mathrm{C}$
$\mathrm{C}$
$\mathrm{C}$
$\mathrm{C}$
$\mathrm{C}$
$\mathrm{C}$
$\mathrm{C}$
$\mathrm{C}$
$\mathrm{C}$

$$
\begin{array}{r}
-1.14939900 \\
1.68993300 \\
2.69978000 \\
2.06043400 \\
4.01293500 \\
3.36690900 \\
4.34749800 \\
0.29638300 \\
-0.19668500 \\
-0.38884200 \\
-1.24946300
\end{array}
$$

$$
\begin{array}{r}
-0.68832200 \\
0.23784600 \\
1.03388600 \\
-1.02127200 \\
0.58652900 \\
-1.48766500 \\
-0.67931800 \\
0.74837600 \\
1.15427300 \\
1.27002800 \\
2.02819200
\end{array}
$$

$-0.56346900$

0.14704800

$-0.40111500$

0.62558200

$-0.50844800$

0.53405500

$-0.03576600$

0.24653300

1.52383000

$-0.90672300$

1.66024600 


$\begin{array}{lrrr}C & -1.45568100 & 2.19934600 & -0.74262300 \\ C & -1.88778600 & 2.54916100 & 0.51550700 \\ F & 2.41660200 & 2.27119700 & -0.82951700 \\ F & 1.14295600 & -1.83444300 & 1.17205900 \\ F & 3.68528300 & -2.70500300 & 0.98790100 \\ F & 5.60525100 & -1.11670800 & -0.12603400 \\ F & 4.95474500 & 1.37060700 & -1.04304600 \\ F & 0.42312700 & 0.69722800 & 2.62297100 \\ F & 0.24663400 & 1.30049100 & -2.10948600 \\ F & -2.04347300 & 2.71001000 & -1.83196600 \\ F & -1.71479400 & 2.36289500 & 2.87198800 \\ F & -2.94426300 & 3.35522300 & 0.67280800 \\ C & -2.07873900 & -3.01634100 & 0.23611000 \\ C & -2.09276500 & -2.63571500 & -1.12868600 \\ C & -3.17313400 & -1.85955700 & -1.61767800 \\ C & -4.23575500 & -1.52337800 & -0.76476400 \\ C & -4.23090000 & -1.94274800 & 0.56682000 \\ C & -3.14778200 & -2.67054600 & 1.07049800 \\ H & -1.26186800 & -3.62041400 & 0.61577300 \\ H & -1.35374800 & -3.03946400 & -1.81523700 \\ H & -3.20909600 & -1.58843700 & -2.66745700 \\ H & -5.06850700 & -0.94477700 & -1.15153300 \\ H & -5.06393200 & -1.69311100 & 1.21599700 \\ H & -3.13717400 & -2.98103900 & 2.11036500\end{array}$

\section{7) $\left[\mathrm{Pd}\left(\mathrm{C}_{6} \mathrm{H}_{6}\right)_{2}\right]$ (22)}

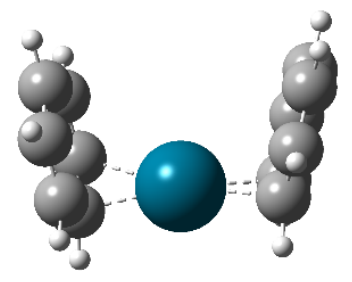

Number of imaginary frequencies $=0$

$$
\mathrm{E}_{\text {total }}=-592.51722559 \mathrm{a} . \mathrm{u}
$$

$\mathrm{G}_{\text {correction }}=0.158618 \mathrm{a} . \mathrm{u}$

\section{Cartesian coordinates:}

$\mathrm{Pd}$
$\mathrm{C}$
$\mathrm{C}$
$\mathrm{C}$
$\mathrm{C}$
$\mathrm{C}$
$\mathrm{C}$
$\mathrm{C}$
$\mathrm{C}$
$\mathrm{C}$
$\mathrm{C}$
$\mathrm{C}$
$\mathrm{C}$
$\mathrm{H}$
$\mathrm{H}$

$$
\begin{array}{r}
-0.01545600 \\
2.09076200 \\
2.34825700 \\
2.12105300 \\
2.64267000 \\
2.40950400 \\
2.67360200 \\
-2.27790100 \\
-2.19399900 \\
-2.24554900 \\
-2.44027100 \\
-2.57317500 \\
-2.47166700 \\
-2.27320100 \\
-2.25651000
\end{array}
$$

$-0.70586400$

$-0.79849600$

0.44093900

$-0.86363300$

1. 57240700

0.31212900

1.50780900

$-0.30641900$

$-0.99432200$

$-0.24402400$

1.14261000

1.79910500

1.08120300

$-0.87042900$

$-2.07921200$
0.00436100

0.76968300

1.41138500

$-0.65194600$

0.66479000

$-1.39315500$

$-0.74143200$

1.19002800

$-0.04837700$

$-1.25163000$

$-1.20868900$

0.01763900

1.21196100

2.11689500

$-0.07710800$ 


$\begin{array}{rrrr}\mathrm{H} & -2.21231200 & -0.76009600 & -2.20547200 \\ \mathrm{H} & -2.50005100 & 1.70354700 & -2.13622000 \\ \mathrm{H} & -2.74335200 & 2.87092800 & 0.04275300 \\ \mathrm{H} & -2.55606100 & 1.59445600 & 2.16487400 \\ \mathrm{H} & 2.07033300 & -1.71111900 & 1.35882800 \\ \mathrm{H} & 2.12364600 & -1.82646000 & -1.15545800 \\ \mathrm{H} & 2.45117900 & 0.25721100 & -2.47678800 \\ \mathrm{H} & 2.90968400 & 2.39762700 & -1.31723200 \\ \mathrm{H} & 2.85519400 & 2.51163800 & 1.16652800 \\ \mathrm{H} & 2.34273400 & 0.48578500 & 2.49626900\end{array}$

\section{8) $\left(\mathrm{C}_{6} \mathrm{~F}_{5}\right)_{2}$}

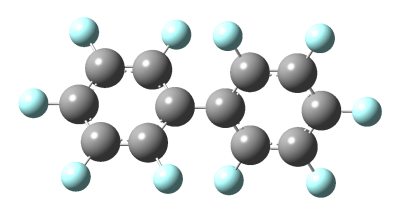

Number of imaginary frequencies $=0$

$E_{\text {total }}=-1455.61597898$ a.u

$\mathrm{G}_{\text {correction }}=0.053206 \mathrm{a} . \mathrm{u}$

\section{Cartesian coordinates:}

$C$
$C$
$C$
$C$
$C$
$C$
$C$
$C$
$C$
$C$
$C$
$C$
$C$
$F$
$F$
$F$
$F$
$F$
$F$
$F$
$F$
$F$
$F$
$F$

0.73804500

1.46466400

1.46467200

2.85505900

2.85507100

3.55159100

$-0.73804600$

$-1.46467100$

$-1.46466500$

$-2.85507400$

$-2.85505600$

$-3.55158800$

0.82124900

0.82127400

3.52354200

4.88545600

3.52352900

$-0.82127600$

$-0.82124400$

$-3.52353800$

$-3.52353600$

$-4.88545500$
0.00001000

$-1.06542100$

1.06543100

$-1.07802400$

1.07802100

$-0.00000200$

0.00001000

1.06542200

$-1.06542000$

1.07801700

$-1.07801900$

0.00000100

$-2.10397800$

2.10398700

2.10938900

$-0.00001400$

$-2.10939800$

2.10397800

$-2.10396400$

$-2.10938000$

2.10938500

$-0.00002200$
0.00000000

$-0.54029900$

0.54029800

$-0.53995000$

0.53995000

$-0.00000100$

$-0.00000200$

$-0.54031000$

0.54031000

$-0.53996500$

0.53995900

$-0.00000300$

$-1.08726300$

1.08728100

1.06534900

$-0.00000100$

$-1.06533800$

$-1.08730000$

1.08728700

1.06535800

$-1.06537100$

0.00000500

\section{9) $\left[\mathrm{Pd}\left(\mathrm{C}_{6} \mathrm{~F}_{5}\right)_{2}\right](\mathbf{2 3})$}




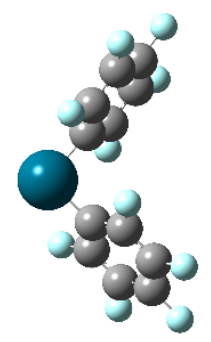

Number of imaginary frequencies $=0$

$E_{\text {total }}=-1583.52090997 \mathrm{a} . \mathrm{u}$

$\mathrm{G}_{\text {correction }}=0.046148 \mathrm{a} . \mathrm{u}$

Cartesian coordinates:

$\begin{array}{lrrr}\text { Pd } & -0.00514400 & 2.04145700 & 0.11334000 \\ \text { C } & -1.34404800 & 0.60803900 & 0.23149100 \\ \text { C } & -2.31657200 & 0.50550900 & -0.75995300 \\ \text { C } & -1.38211800 & -0.31383200 & 1.27346600 \\ \text { C } & -3.31654300 & -0.46142500 & -0.71035700 \\ \text { C } & -2.37084200 & -1.29211100 & 1.34781500 \\ \text { C } & -3.33960800 & -1.36494400 & 0.34971800 \\ \text { C } & 1.34052300 & 0.63804800 & -0.16412100 \\ \text { C } & 2.29187900 & 0.41015100 & 0.82642900 \\ \text { C } & 1.40408400 & -0.13894900 & -1.31663900 \\ \text { C } & 3.29802700 & -0.53902000 & 0.67259800 \\ \text { C } & 2.39976500 & -1.09620100 & -1.49703300 \\ \text { C } & 3.34787200 & -1.29579000 & -0.49618300 \\ \text { F } & -2.32237300 & 1.36095100 & -1.80294100 \\ \text { F } & -0.45821900 & -0.28668900 & 2.25190600 \\ \text { F } & -2.39970100 & -2.16091700 & 2.36665100 \\ \text { F } & -4.29690600 & -2.29540100 & 0.41373300 \\ \text { F } & -4.25214500 & -0.53186300 & -1.66593300 \\ \text { F } & 2.26689100 & 1.12270800 & 1.97107100 \\ \text { F } & 0.49915000 & 0.01390800 & -2.30140200 \\ \text { F } & 2.45563100 & -1.82398200 & -2.62001200 \\ \text { F } & 4.21398800 & -0.73209200 & 1.63036200 \\ \text { F } & 4.31169500 & -2.20705300 & -0.66088500\end{array}$

\section{0) $\mathbf{C}_{6} \mathbf{H}_{6}$}

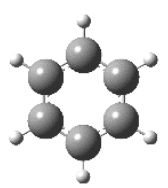

Number of imaginary frequencies $=0$

$E_{\text {total }}=-232.27814567$ a.u

$\mathrm{G}_{\text {correction }}=0.073222 \mathrm{a} . \mathrm{u}$

\section{Cartesian coordinates:}

$\begin{array}{lrrr}\text { C } & 1.06383300 & -0.90425300 & -0.00000500 \\ \text { C } & -0.25133100 & -1.37329800 & -0.00006700 \\ \text { C } & -1.31506700 & -0.46915300 & 0.00005500 \\ \text { C } & -1.06375600 & 0.90434200 & -0.00000800\end{array}$




$\begin{array}{lrrr}\text { C } & 0.25121500 & 1.37331700 & -0.00006100 \\ \text { C } & 1.31510700 & 0.46904700 & 0.00005600 \\ \text { H } & 1.89119200 & -1.60781000 & 0.00006500 \\ \text { H } & -0.44663500 & -2.44167100 & -0.00007700 \\ \text { H } & -2.33801000 & -0.83396700 & 0.00014800 \\ \text { H } & -1.89128800 & 1.60768700 & -0.00000900 \\ \text { H } & 0.44676800 & 2.44163800 & -0.00001400 \\ \text { H } & 2.33796800 & 0.83411400 & 0.00005900\end{array}$

\section{1) $\operatorname{TS}(23-24)$}

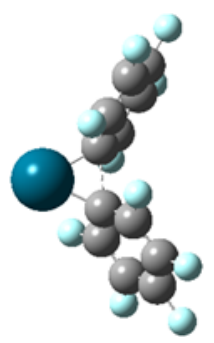

Number of imaginary frequencies $=1\left(i 267.61 \mathrm{~cm}^{-1}\right)$

$E_{\text {total }}=-1583.49220244 \mathrm{a} . \mathrm{u}$

$\mathrm{G}_{\text {correction }}=0.048602 \mathrm{a} . \mathrm{u}$

\section{Cartesian coordinates:}

$\begin{array}{lrrr}\mathrm{Pd} & -0.00006100 & 0.00023900 & 2.16002400 \\ \mathrm{C} & -0.91885400 & -0.12247100 & 0.32900700 \\ \mathrm{C} & -1.84145000 & 0.94429700 & 0.23314800 \\ \mathrm{C} & -1.28209900 & -1.28736200 & -0.37411000 \\ \mathrm{C} & -3.00388000 & 0.87050400 & -0.52280000 \\ \mathrm{C} & -2.43962800 & -1.38023000 & -1.13709700 \\ \mathrm{C} & -3.30552900 & -0.29470400 & -1.22180100 \\ \mathrm{C} & 0.91889400 & 0.12255400 & 0.32902900 \\ \mathrm{C} & 1.84148200 & -0.94424200 & 0.23340900 \\ \mathrm{C} & 1.28215400 & 1.28729000 & -0.37433100 \\ \mathrm{C} & 3.00391500 & -0.87061400 & -0.52255300 \\ \mathrm{C} & 2.43967400 & 1.37998700 & -1.13735100 \\ \mathrm{C} & 3.30557100 & 0.29443900 & -1.22181000 \\ \mathrm{~F} & -1.66969200 & 2.07649400 & 0.94462800 \\ \mathrm{~F} & -0.48075000 & -2.36343900 & -0.37168400 \\ \mathrm{~F} & -2.72189600 & -2.50953400 & -1.79884100 \\ \mathrm{~F} & -4.42348500 & -0.37734600 & -1.94667200 \\ \mathrm{~F} & -3.85923500 & 1.90039300 & -0.55167900 \\ \mathrm{~F} & 1.66968300 & -2.07631200 & 0.94507200 \\ \mathrm{~F} & 0.48080000 & 2.36336500 & -0.37215500 \\ \mathrm{~F} & 2.72193100 & 2.50913700 & -1.79936100 \\ \mathrm{~F} & 3.85926200 & -1.90051600 & -0.55122100 \\ \mathrm{~F} & 4.42352700 & 0.37690700 & -1.94670100 \\ \mathrm{~F} & & \end{array}$

42) Intermediate-24 


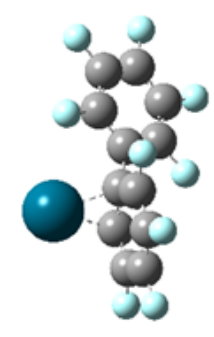

Number of imaginary frequencies $=0$

$E_{\text {total }}=-1583.53477720$ a.u

$\mathrm{G}_{\text {correction }}=0.049145 \mathrm{a} . \mathrm{u}$

Cartesian coordinates:

\begin{tabular}{|c|c|c|c|}
\hline $\mathrm{Pd}$ & 1.19815500 & -1.49563800 & -1.12346400 \\
\hline $\mathrm{C}$ & -1.05092900 & 0.14037800 & 0.15902200 \\
\hline C & -1.87166300 & -0.97530700 & 0.33562500 \\
\hline C & -1.68006700 & 1.37593600 & -0.01534600 \\
\hline $\mathrm{C}$ & -3.25829600 & -0.88115200 & 0.30852600 \\
\hline $\mathrm{C}$ & -3.06593000 & 1.49486500 & -0.05262800 \\
\hline $\mathrm{C}$ & -3.85709400 & 0.36147600 & 0.11402000 \\
\hline C & 0.43357300 & 0.04493000 & 0.20144600 \\
\hline C & 1.24560700 & 0.58905000 & -0.8646730 \\
\hline $\mathrm{C}$ & 1.06594200 & -0.11538900 & 1.4728200 \\
\hline $\mathrm{C}$ & 2.58442700 & 1.00491300 & -0.59549600 \\
\hline C & 2.36762300 & 0.26643200 & 1.6982680 \\
\hline C & 3.13333200 & 0.82537100 & 0.6531080 \\
\hline $\mathrm{F}$ & -1.32648500 & -2.19195600 & 0.5037890 \\
\hline $\mathrm{F}$ & -0.94413000 & 2.48812800 & -0.1401270 \\
\hline $\mathrm{F}$ & -3.63984100 & 2.68929900 & -0.2277090 \\
\hline $\mathrm{F}$ & -5.18713100 & 0.46515300 & 0.0870150 \\
\hline $\mathrm{F}$ & -4.01634300 & -1.97160900 & 0.4635900 \\
\hline $\mathrm{F}$ & 0.64107900 & 1.20362700 & -1.9188710 \\
\hline $\mathrm{F}$ & 0.33928500 & -0.60325200 & 2.4904860 \\
\hline $\mathrm{F}$ & 2.94316300 & 0.08044400 & 2.8939650 \\
\hline $\mathrm{F}$ & 3.29347800 & 1.57789000 & -1.5760910 \\
\hline $\mathrm{F}$ & 4.40867500 & 1.15231100 & 0.8896430 \\
\hline
\end{tabular}

43) $\left[\mathrm{Pd}\left(\mathrm{C}_{6} \mathrm{H}_{5} \mathrm{CH}_{3}\right)\left(\mathrm{C}_{6} \mathrm{~F}_{5}\right)_{2}\right]$ (25)

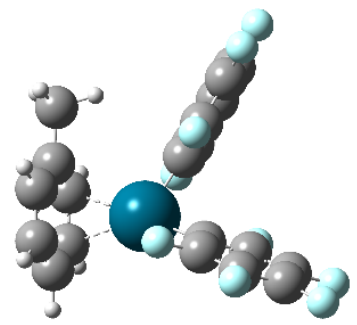

Number of imaginary frequencies $=0$

$\mathrm{E}_{\text {total }}=-1855.16575508 \mathrm{a} . \mathrm{u}$

$\mathrm{G}_{\text {correction }}=0.165795 \mathrm{a} . \mathrm{u}$

Cartesian coordinates: 


\begin{tabular}{|c|c|c|c|}
\hline C & -1.46447300 & 0.23001000 & -0.20820300 \\
\hline $\mathrm{C}$ & -1.72645000 & -0.64488900 & -1.25522300 \\
\hline $\mathrm{C}$ & -2.48469500 & 0.42998300 & 0.71506000 \\
\hline $\mathrm{C}$ & -2.95359300 & -1.29013400 & -1.39447500 \\
\hline $\mathrm{C}$ & -3.72305900 & -0.19562000 & 0.60819800 \\
\hline $\mathrm{C}$ & -3.95584600 & -1.06412400 & -0.45525300 \\
\hline $\mathrm{C}$ & 1.11342100 & -0.55535300 & 0.20269000 \\
\hline C & 0.85615700 & -1.43186200 & 1.25033800 \\
\hline $\mathrm{C}$ & 2.07212600 & -0.94951300 & -0.72426900 \\
\hline C & 1.53073100 & -2.64335400 & 1.38812500 \\
\hline C & 2.76883700 & -2.14910200 & -0.61700800 \\
\hline $\mathrm{C}$ & 2.49126600 & -3.00325000 & 0.44696900 \\
\hline $\mathrm{F}$ & -0.78745200 & -0.89904500 & -2.19226500 \\
\hline $\mathrm{F}$ & -2.29677100 & 1.26227300 & 1.76639700 \\
\hline $\mathrm{F}$ & -4.68974300 & 0.02403300 & 1.51297900 \\
\hline $\mathrm{F}$ & -5.14284600 & -1.67303600 & -0.57798800 \\
\hline $\mathrm{F}$ & -3.18153800 & -2.12164200 & -2.42276700 \\
\hline $\mathrm{F}$ & -0.06363800 & -1.12893500 & 2.19115000 \\
\hline F & 2.37224300 & -0.15310700 & -1.78003200 \\
\hline F & 3.69592200 & -2.49280400 & -1.52419900 \\
\hline F & 1.27006500 & -3.46320200 & 2.41743800 \\
\hline F & 3.15320700 & -4.16117400 & 0.56960500 \\
\hline $\mathrm{C}$ & 0.29470900 & 3.56838500 & 1.19191300 \\
\hline $\mathrm{C}$ & -0.34479300 & 3.77121900 & -0.04445700 \\
\hline $\mathrm{C}$ & 0.36877100 & 3.57913900 & -1.24792500 \\
\hline $\mathrm{C}$ & 1.68480400 & 3.11110500 & -1.21303100 \\
\hline $\mathrm{C}$ & 2.31120600 & 2.81007900 & 0.02128700 \\
\hline $\mathrm{C}$ & 1.60945200 & 3.07910700 & 1.22130600 \\
\hline $\mathrm{H}$ & -0.24239200 & 3.74007900 & 2.11738500 \\
\hline $\mathrm{H}$ & -1.37581900 & 4.10521500 & -0.07300700 \\
\hline $\mathrm{H}$ & 2.09040600 & 2.87771600 & 2.17259300 \\
\hline $\mathrm{H}$ & -0.11691900 & 3.76481700 & -2.19927900 \\
\hline $\mathrm{H}$ & 2.22094400 & 2.92881000 & -2.13790200 \\
\hline $\mathrm{C}$ & 3.71394200 & 2.26287600 & 0.04971300 \\
\hline $\mathrm{H}$ & 3.94541400 & 1.82041000 & 1.02086300 \\
\hline $\mathrm{H}$ & 3.84266700 & 1.50035200 & -0.72105900 \\
\hline $\mathrm{H}$ & 4.43853600 & 3.06245300 & -0.14054500 \\
\hline
\end{tabular}

\section{4) $\mathrm{TS}(\mathbf{2 5 - 2 6 )}$}

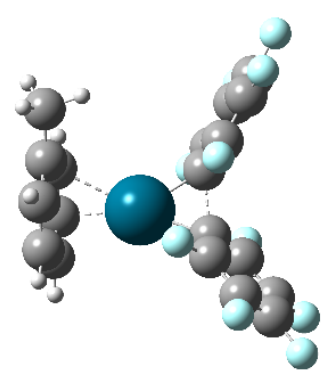

Number of imaginary frequencies $=1\left(i 259.13 \mathrm{~cm}^{-1}\right)$

$\mathrm{E}_{\text {total }}=-1855.13405430 \mathrm{a} . \mathrm{u}$

$\mathrm{G}_{\text {correction }}=0.164140 \mathrm{a} . \mathrm{u}$

Cartesian coordinates: 


$\begin{array}{lrrr}\text { Pd } & -0.75822800 & -0.85654300 & -0.67552700 \\ \mathrm{C} & 1.13933700 & -0.24664000 & -0.15379400 \\ \mathrm{C} & 2.16626200 & 0.20197500 & -1.00490900 \\ \mathrm{C} & 1.56823300 & -1.06356400 & 0.91708500 \\ \mathrm{C} & 3.50689400 & -0.09946700 & -0.79940200 \\ \mathrm{C} & 2.90216800 & -1.37884200 & 1.13768300 \\ \mathrm{C} & 3.88427700 & -0.88739200 & 0.28293400 \\ \mathrm{C} & -0.20392100 & 1.00994200 & 0.00162300 \\ \mathrm{C} & -0.29923500 & 1.50254100 & 1.31608500 \\ \mathrm{C} & -0.51005900 & 1.94903400 & -1.00900000 \\ \mathrm{C} & -0.63240600 & 2.81914900 & 1.61079300 \\ \mathrm{C} & -0.84593900 & 3.26791100 & -0.73572400 \\ \mathrm{C} & -0.90021500 & 3.71478800 & 0.58147000 \\ \mathrm{~F} & 1.88672000 & 0.98961800 & -2.05552100 \\ \mathrm{~F} & 0.67873000 & -1.65719500 & 1.73826100 \\ \mathrm{~F} & 3.24853700 & -2.18903100 & 2.14754700 \\ \mathrm{~F} & 5.17042900 & -1.18642900 & 0.48685500 \\ \mathrm{~F} & 4.44032300 & 0.37182000 & -1.63713900 \\ \mathrm{~F} & -0.02712300 & 0.70779400 & 2.36386500 \\ \mathrm{~F} & -0.57528900 & 1.57990000 & -2.30428400 \\ \mathrm{~F} & -1.16287300 & 4.10682600 & -1.73159800 \\ \mathrm{~F} & -0.69009000 & 3.23086000 & 2.88475900 \\ \mathrm{~F} & -1.22668300 & 4.98175700 & 0.85296600 \\ \mathrm{C} & -1.78079400 & -3.80429800 & 0.05554400 \\ \mathrm{C} & -1.51085000 & -3.31905500 & -1.23079600 \\ \mathrm{C} & -2.24997400 & -2.22038600 & -1.72763100 \\ \mathrm{C} & -3.20131400 & -1.58869600 & -0.89353900 \\ \mathrm{C} & -3.45808800 & -2.07249800 & 0.40487500 \\ \mathrm{C} & -2.75413700 & -3.19860900 & 0.85222700 \\ \mathrm{H} & -1.23352500 & -4.66307200 & 0.43063100 \\ \mathrm{H} & -0.78715100 & -3.81671000 & -1.86692200 \\ \mathrm{H} & -2.95788100 & -3.59232400 & 1.84345300 \\ \mathrm{H} & -2.15494200 & -1.92089900 & -2.76706600 \\ \mathrm{H} & -3.78562600 & -0.76044800 & -1.28237500 \\ \mathrm{C} & -4.45038700 & -1.37257400 & 1.29595900 \\ \mathrm{H} & -5.27560900 & -0.94551400 & 0.71919500 \\ \mathrm{H} & -4.86479300 & -2.05409600 & 2.04319600 \\ \mathrm{H} & -3.96525100 & -0.54915100 & 1.83384600 \\ & & & \\ & & & \\ & & & \end{array}$

\section{5) Intermediate-26}

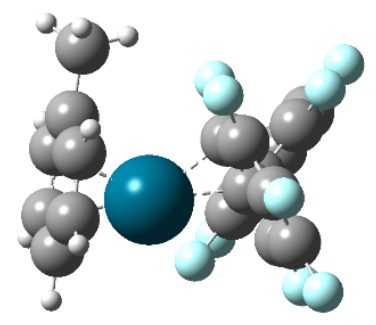

Number of imaginary frequencies $=0$

$$
\mathrm{E}_{\text {total }}=-1855.17850248 \mathrm{a} . \mathrm{u}
$$

$\mathrm{G}_{\text {correction }}=0.167396 \mathrm{a} . \mathrm{u}$

\section{Cartesian coordinates:}




$\begin{array}{lrrr}\text { C } & -0.48052600 & 0.74213700 & -0.19734700 \\ \text { C } & -0.17953700 & 1.68110900 & -1.23254900 \\ \text { C } & 0.45934500 & 0.66411900 & 0.89022500 \\ \text { C } & 0.92322700 & 2.50107600 & -1.18487100 \\ \text { C } & 1.56885000 & 1.55335800 & 0.94346800 \\ \text { C } & 1.80833800 & 2.42914600 & -0.08810800 \\ \text { C } & -1.88040400 & 0.26741300 & -0.03579800 \\ \text { C } & -2.68115100 & 0.75524200 & 1.00005000 \\ \text { C } & -2.46084200 & -0.65120500 & -0.91273200 \\ \text { C } & -3.99362700 & 0.32913500 & 1.17788700 \\ \text { C } & -3.77226000 & -1.08661200 & -0.76101200 \\ \text { C } & -4.54131500 & -0.59324900 & 0.29048500 \\ \text { F } & -1.03524800 & 1.79126300 & -2.26061700 \\ \text { F } & 0.06774800 & 0.13507400 & 2.08149900 \\ \text { F } & 2.40831000 & 1.47937700 & 1.98529800 \\ \text { F } & 2.90539400 & 3.19482300 & -0.09036700 \\ \text { F } & 1.20116200 & 3.34486300 & -2.18921500 \\ \text { F } & -2.18960100 & 1.66784900 & 1.84903700 \\ \text { F } & -1.74263300 & -1.16114900 & -1.92779600 \\ \text { F } & -4.29565400 & -1.97642200 & -1.61102300 \\ \text { F } & -4.73381600 & 0.81151300 & 2.18137000 \\ \text { F } & -5.80147500 & -1.00425200 & 0.44641800 \\ \text { C } & 4.35505700 & -0.93308400 & -0.79487900 \\ \text { C } & 3.46548600 & -1.70200100 & -1.53790700 \\ \text { C } & 2.49458100 & -2.49063300 & -0.87218400 \\ \text { C } & 2.41834800 & -2.44870400 & 0.54207800 \\ \text { C } & 3.32308200 & -1.65702800 & 1.29229500 \\ \text { C } & 4.28981000 & -0.92339700 & 0.60695700 \\ \text { H } & 5.11222700 & -0.34264400 & -1.30134500 \\ \text { H } & 3.53217100 & -1.73372700 & -2.62043100 \\ \text { H } & 4.99654400 & -0.32216600 & 1.17136200 \\ \text { H } & 1.91524200 & -3.22559100 & -1.42670000 \\ \text { H } & 1.74530000 & -3.12218000 & 1.06509700 \\ \text { C } & 3.20354900 & -1.59703600 & 2.79266700 \\ H & 4.10544400 & -1.17894200 & 3.24645000 \\ \text { H } & 2.35958100 & -0.96177700 & 3.08077100 \\ \text { H } & 3.02978600 & -2.58984500 & 3.22001600\end{array}$

\section{6) $\left[\mathrm{Pd}\left(\mathrm{C}_{6} \mathrm{H}_{5} \mathrm{CH}_{3}\right)_{2}\right](27)$}

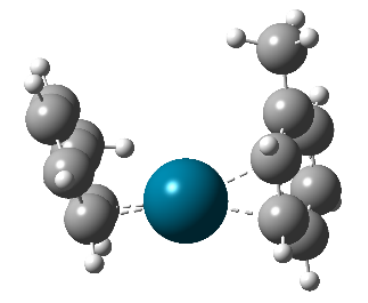

Number of imaginary frequencies $=0$

$\mathrm{E}_{\text {total }}=-671.17130088 \mathrm{a} \cdot \mathrm{u}$

$\mathrm{G}_{\text {correction }}=0.211236 \mathrm{a} . \mathrm{u}$

\section{Cartesian coordinates:}

$\mathrm{Pd}$

C
-0.00000100
1.61821600
1.04075700

0.10292700
$-0.00000400$

$-1.27948500$ 
1.80801500

2.15468600

2. 55351400

2.88331000

3.08659000

$-3.08660600$

$-2.88332300$

$-2.15468300$

$-1.61820100$

$-1.80800600$

$-2.55351900$

$-3.66385300$

$-3.30904100$

$-2.72578500$

$-2.17277200$

$-1.20950400$

$-1.19567500$

$-1.55596200$

$-0.10411600$

$-1.42358100$

1.20952800

2.17277800

3.30902000

3.66382600

2.72577900

1.19568800

1.55604100

0.10413400

1. 42352100
$-1.21187900$

1.22371500

$-1.37511200$

1.01821400

$-0.26857100$

$-0.26857300$

1.01821100

1.22371100

0.10292100

$-1.21188400$

$-1.37511500$

$-0.42820200$

1.87176200

$-2.37764400$

2.19909700

0.22240100

$-2.38391800$

$-3.33394200$

$-2.36521500$

$-2.35787900$

0.22240900

2.19910300

1.87176500

$-0.42820100$

$-2.37764200$

$-2.38391200$

$-3.33393700$

$-2.36526000$

$-2.35782300$
$-0.76912800$

$-0.59378200$

0.39337300

0.60789300

1.08209300

$-1.08207800$

$-0.60787700$

0.59378800

1.27948200

0.76912400

$-0.39336800$

$-1.98791200$

$-1.12601700$

$-0.77609200$

1.07383000

2. 27975700

1. 49029200

1.08700800

1.39096600

2.56169300

$-2.27976400$

$-1.07382000$

1.12604100

1.98793300

0.77609500

$-1.49030000$

$-1.08707500$

$-1.39090200$

$-2.56171500$

\section{7) $\left(\mathrm{C}_{6} \mathrm{~F}_{5}\right)_{2}$}

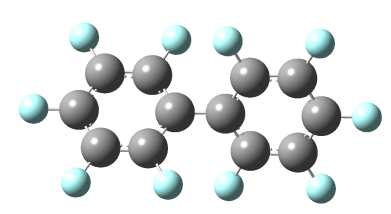

Number of imaginary frequencies $=0$

$\mathrm{E}_{\text {total }}=-1455.61604973 \mathrm{a} . \mathrm{u}$

$\mathrm{G}_{\text {correction }}=0.053196 \mathrm{a} . \mathrm{u}$

\section{Cartesian coordinates:}

C
$C$
$C$
$C$
$C$
$C$
$C$
$C$
$C$
$C$
$C$
$C$
$C$

$$
\begin{array}{r}
0.73804600 \\
1.46468000 \\
1.46468800 \\
2.85505700 \\
2.85506900 \\
3.55158300 \\
-0.73804700 \\
-1.46468800 \\
-1.46468000 \\
-2.85507300 \\
-2.85505300
\end{array}
$$

0.00001000

$-1.06542000$

1.06543100

$-1.07801700$

1.07801500

$-0.00000200$

0.00001100

1.06542100

$-1.06541900$

1.07801100

$-1.07801300$
$-0.00000100$

$-0.54025600$

0.54025500

$-0.53990600$

0.53990600

$-0.00000100$

$-0.00000200$

$-0.54026700$

0.54026600

$-0.53992000$

0.53991500 


$\begin{array}{lrrr}C & -3.55158100 & 0.00000000 & -0.00000300 \\ F & 0.82129100 & -2.10404700 & -1.08717800 \\ F & 0.82131600 & 2.10405600 & 1.08719800 \\ F & 3.52355900 & 2.10942900 & 1.06527600 \\ F & 4.88547700 & -0.00001400 & 0.00000000 \\ F & 3.52354600 & -2.10943800 & -1.06526400 \\ F & -0.82131900 & 2.10404700 & -1.08721700 \\ F & -0.82128500 & -2.10403300 & 1.08720200 \\ F & -3.52355400 & -2.10942000 & 1.06528500 \\ F & -3.52355400 & 2.10942300 & -1.06529700 \\ F & -4.88547600 & -0.00002200 & 0.00000600\end{array}$

\section{8) $\left[\mathrm{Pd}\left(\mathrm{C}_{6} \mathrm{~F}_{5}\right)_{2}\right](\mathbf{2 8})$}

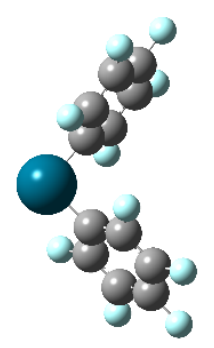

Number of imaginary frequencies $=0$

$E_{\text {total }}=-1583.52151770 \mathrm{a} \cdot \mathrm{u}$

$\mathrm{G}_{\text {correction }}=0.046166 \mathrm{a} . \mathrm{u}$

\section{Cartesian coordinates:}

\begin{tabular}{|c|c|c|c|}
\hline $\mathrm{Pd}$ & -0.00509100 & 2.04249000 & 0.11226900 \\
\hline $\mathrm{C}$ & -1.34342100 & 0.60846300 & 0.23083700 \\
\hline C & -2.31582800 & 0.50443200 & -0.76053800 \\
\hline C & -1.38180300 & -0.31234300 & 1.27372600 \\
\hline $\mathrm{C}$ & -3.31546700 & -0.46281200 & -0.71024000 \\
\hline C & -2.37015100 & -1.29091100 & 1.34894100 \\
\hline C & -3.33861800 & -1.36521300 & 0.35072500 \\
\hline C & 1.34001000 & 0.63825100 & -0.16437500 \\
\hline $\mathrm{C}$ & 2.29135100 & 0.41016300 & 0.8261060 \\
\hline C & 1.40368100 & -0.13921800 & -1.31655700 \\
\hline C & 3.29706000 & -0.53955600 & 0.67280700 \\
\hline C & 2.39885200 & -1.09705100 & -1.4965620 \\
\hline C & 3.34675400 & -1.29680900 & -0.4956110 \\
\hline $\mathrm{F}$ & -2.32194100 & 1.35867600 & -1.8046370 \\
\hline $\mathrm{F}$ & -0.45830800 & -0.28388600 & 2.2526930 \\
\hline $\mathrm{F}$ & -2.39897800 & -2.15862100 & 2.3688360 \\
\hline $\mathrm{F}$ & -4.29567200 & -2.29601600 & 0.4154470 \\
\hline $\mathrm{F}$ & -4.25079100 & -0.53460700 & -1.6661270 \\
\hline $\mathrm{F}$ & 2.26681800 & 1.12307000 & 1.9706620 \\
\hline $\mathrm{F}$ & 0.49910000 & 0.01371400 & -2.3018120 \\
\hline $\mathrm{F}$ & 2.45447300 & -1.82525400 & -2.6193940 \\
\hline $\mathrm{F}$ & 4.21283500 & -0.73271300 & 1.6308700 \\
\hline $\mathrm{F}$ & 4.31020600 & -2.20868900 & -0.6598630 \\
\hline
\end{tabular}

\section{9) $\mathbf{C}_{6} \mathbf{H}_{5} \mathrm{CH}_{3}$}




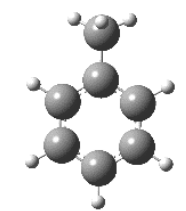

Number of imaginary frequencies $=0$

$\mathrm{E}_{\text {total }}=-271.60402628 \mathrm{a} . \mathrm{u}$

$\mathrm{G}_{\text {correction }}=0.097438 \mathrm{a} . \mathrm{u}$

\section{Cartesian coordinates:}

$\begin{array}{lrrr}\text { C } & 1.90333900 & -0.00011500 & 0.00841600 \\ \mathrm{C} & 1.19968700 & -1.20522300 & 0.00196600 \\ \mathrm{C} & -0.19510300 & -1.20219900 & -0.00926800 \\ \mathrm{C} & -0.91319500 & 0.00016700 & -0.01173900 \\ \mathrm{C} & -0.19490300 & 1.20231500 & -0.00923900 \\ \mathrm{C} & 1.19996300 & 1.20507100 & 0.00199300 \\ \mathrm{H} & 2.98899800 & -0.00024100 & 0.01396400 \\ \mathrm{H} & 1.73676100 & -2.14918900 & 0.00143400 \\ \mathrm{H} & -0.73601800 & -2.14497100 & -0.01807100 \\ \mathrm{H} & -0.73561900 & 2.14519400 & -0.01800500 \\ \mathrm{H} & 1.73718900 & 2.14895000 & 0.00148400 \\ \mathrm{C} & -2.42199800 & 0.00006900 & 0.00994500 \\ \mathrm{H} & -2.80111700 & -0.00814400 & 1.03923000 \\ \mathrm{H} & -2.82842500 & 0.88987700 & -0.47929800 \\ \mathrm{H} & -2.82850700 & -0.88197800 & -0.49319000\end{array}$

\section{0) $\mathbf{T S}(\mathbf{2 8 - 2 9 )}$}

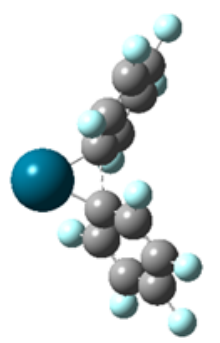

Number of imaginary frequencies $=1\left(i 266.85 \mathrm{~cm}^{-1}\right)$

$\mathrm{E}_{\text {total }}=-1583.49262377 \mathrm{a} . \mathrm{u}$

$\mathrm{G}_{\text {correction }}=0.048596 \mathrm{a} . \mathrm{u}$

\section{Cartesian coordinates:}

$\begin{array}{ll}\mathrm{Pd} & -0.00006800 \\ \mathrm{C} & -0.91779200 \\ \mathrm{C} & -1.84055200 \\ \mathrm{C} & -1.28206300 \\ \mathrm{C} & -3.00345500 \\ \mathrm{C} & -2.44006000 \\ \mathrm{C} & -3.30585600 \\ \mathrm{C} & 0.91783500 \\ \mathrm{C} & 1.84058600 \\ \mathrm{C} & 1.28212600 \\ \mathrm{C} & 3.00349000 \\ \mathrm{C} & 2.44011300\end{array}$

0.00030400

$-0.12225000$

0.94455400

$-1.28712600$

0.87081800

$-1.37996000$

$-0.29437900$

0.12235600

$-0.94448300$

1.28703600

$-0.87096300$

1.37964700
2.15988600

0.32824600

0.23258200

$-0.37456900$

$-0.52257300$

$-1.13678000$

$-1.22120200$

0.32826800

0.23290700

$-0.37485900$

$-0.52226800$

$-1.13711100$ 


$\begin{array}{lrrr}C & 3.30590100 & 0.29403700 & -1.22122400 \\ F & -1.66854600 & 2.07702000 & 0.94376100 \\ F & -0.48098200 & -2.36351500 & -0.37270400 \\ F & -2.72290300 & -2.50943000 & -1.79815200 \\ F & -4.42435200 & -0.37694000 & -1.94538500 \\ F & -3.85873500 & 1.90089800 & -0.55095100 \\ F & 1.66853600 & -2.07677900 & 0.94433800 \\ F & 0.48104400 & 2.36342400 & -0.37330600 \\ F & 2.72294900 & 2.50892400 & -1.79881600 \\ F & 3.85876000 & -1.90106000 & -0.55037500 \\ F & 4.42439500 & 0.37637700 & -1.94543600\end{array}$

\section{1) Intermediate-29}

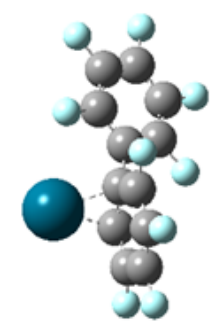

Number of imaginary frequencies $=0$

$E_{\text {total }}=-1583.53510775 \mathrm{a} . \mathrm{u}$

$\mathrm{G}_{\text {correction }}=0.049147 \mathrm{a} . \mathrm{u}$

\section{Cartesian coordinates:}

\begin{tabular}{|c|c|c|c|}
\hline $\mathrm{Pd}$ & 1.19825800 & -1.49613700 & -1.12326700 \\
\hline $\mathrm{C}$ & -1.05080800 & 0.13981600 & 0.15875300 \\
\hline $\mathrm{C}$ & -1.87215200 & -0.97528500 & 0.33619700 \\
\hline $\mathrm{C}$ & -1.67940800 & 1.37555100 & -0.01627000 \\
\hline $\mathrm{C}$ & -3.25872800 & -0.88045800 & 0.30907800 \\
\hline $\mathrm{C}$ & -3.06520300 & 1.49513700 & -0.05362300 \\
\hline $\mathrm{C}$ & -3.85693800 & 0.36229100 & 0.11375200 \\
\hline $\mathrm{C}$ & 0.43368200 & 0.04353100 & 0.20109600 \\
\hline C & 1.24603700 & 0.58706400 & -0.86532700 \\
\hline $\mathrm{C}$ & 1.06575100 & -0.11534800 & 1.47294300 \\
\hline $\mathrm{C}$ & 2.58455600 & 1.00410100 & -0.59556500 \\
\hline $\mathrm{C}$ & 2.36698700 & 0.26738900 & 1.6986420 \\
\hline C & 3.13291900 & 0.82593600 & 0.6533140 \\
\hline $\mathrm{F}$ & -1.32766800 & -2.19212500 & 0.5053290 \\
\hline $\mathrm{F}$ & -0.94303300 & 2.48743300 & -0.1416500 \\
\hline $\mathrm{F}$ & -3.63853600 & 2.68980400 & -0.2294350 \\
\hline $\mathrm{F}$ & -5.18697400 & 0.46660700 & 0.0866970 \\
\hline $\mathrm{F}$ & -4.01732800 & -1.97048100 & 0.4649450 \\
\hline $\mathrm{F}$ & 0.64157400 & 1.20232100 & -1.9195370 \\
\hline $\mathrm{F}$ & 0.33885900 & -0.60272100 & 2.4908040 \\
\hline $\mathrm{F}$ & 2.94226800 & 0.08266900 & 2.8947890 \\
\hline $\mathrm{F}$ & 3.29383500 & 1.57665200 & -1.5764100 \\
\hline $\mathrm{F}$ & 4.40810700 & 1.15361600 & 0.8902830 \\
\hline
\end{tabular}

\section{2) $\left[\mathrm{Pd}\left(\mathrm{C}_{6} \mathrm{H}_{4}\left(\mathrm{CH}_{3}\right)_{2}\right)\left(\mathrm{C}_{6} \mathrm{~F}_{5}\right)_{2}\right]$ (30)}




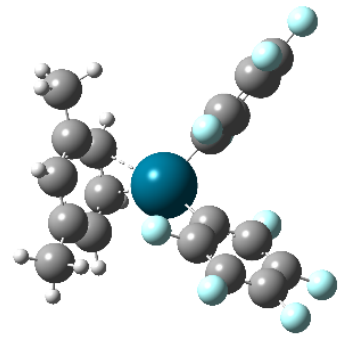

Number of imaginary frequencies $=0$

$\mathrm{E}_{\text {total }}=-1894.49387213 \mathrm{a} . \mathrm{u}$

$\mathrm{G}_{\text {correction }}=0.192649 \mathrm{a} . \mathrm{u}$

\section{Cartesian coordinates:}

$\begin{array}{lrrr}\text { Pd } & -0.03769000 & 1.08923600 & -0.37389400 \\ \mathrm{C} & -1.36532100 & -0.38929500 & -0.28862400 \\ \mathrm{C} & -1.42257800 & -1.41930700 & -1.22010700 \\ \mathrm{C} & -2.31048700 & -0.41632800 & 0.73103500 \\ \mathrm{C} & -2.38716800 & -2.42284200 & -1.16455900 \\ \mathrm{C} & -3.29088300 & -1.40015400 & 0.81930900 \\ \mathrm{C} & -3.32568000 & -2.41224700 & -0.13632200 \\ \mathrm{C} & 1.33588300 & -0.27532400 & 0.03406400 \\ \mathrm{C} & 1.42586100 & -0.91536900 & 1.26434300 \\ \mathrm{C} & 2.31325800 & -0.57577500 & -0.90791200 \\ \mathrm{C} & 2.44905700 & -1.81343800 & 1.56039300 \\ \mathrm{C} & 3.35031800 & -1.46707900 & -0.64628900 \\ \mathrm{C} & 3.41496800 & -2.09094700 & 0.59707600 \\ \mathrm{~F} & -0.53220900 & -1.47643700 & -2.23450200 \\ \mathrm{~F} & -2.30756600 & 0.53949600 & 1.69298300 \\ \mathrm{~F} & -4.19439500 & -1.38902500 & 1.81181100 \\ \mathrm{~F} & -4.26300200 & -3.36718600 & -0.07088300 \\ \mathrm{~F} & -2.42437300 & -3.39600500 & -2.08771100 \\ \mathrm{~F} & 0.51605900 & -0.67424000 & 2.23248200 \\ \mathrm{~F} & 2.29118900 & 0.01194400 & -2.12562000 \\ \mathrm{~F} & 4.28495100 & -1.73000000 & -1.57249100 \\ \mathrm{~F} & 2.51727700 & -2.40785400 & 2.76127400 \\ \mathrm{~F} & 4.40831600 & -2.94762200 & 0.86826200 \\ \mathrm{C} & -1.28547100 & 3.51154100 & 0.10163700 \\ \mathrm{C} & -1.05011000 & 3.35780900 & -1.28633200 \\ \mathrm{C} & 0.25680500 & 3.21809900 & -1.78108600 \\ \mathrm{C} & 1.33356700 & 3.13495200 & -0.88231500 \\ \mathrm{C} & 1.12269600 & 3.28593000 & 0.51428000 \\ \mathrm{C} & -0.18416800 & 3.51208500 & 0.97815600 \\ \mathrm{H} & 0.42966900 & 3.11794100 & -2.84656600 \\ \mathrm{H} & -0.35499400 & 3.63052200 & 2.04354300 \\ \mathrm{H} & -1.88957500 & 3.37158400 & -1.97317900 \\ \mathrm{H} & 2.33943500 & 2.97623000 & -1.25604300 \\ \mathrm{C} & -2.68628300 & 3.66087800 & 0.63450900 \\ \mathrm{H} & -2.99555500 & 2.74201000 & 1.14032800 \\ \mathrm{H} & -2.73857300 & 4.47458300 & 1.36375500 \\ \mathrm{H} & -3.39770900 & 3.86745600 & -0.16800100 \\ \mathrm{C} & 2.28821700 & 3.20194400 & 1.46341100 \\ \mathrm{H} & 1.95326000 & 3.18911100 & 2.50234100 \\ \mathrm{H} & 2.86582100 & 2.29163900 & 1.27714600\end{array}$




\section{3) TS(30-31)}

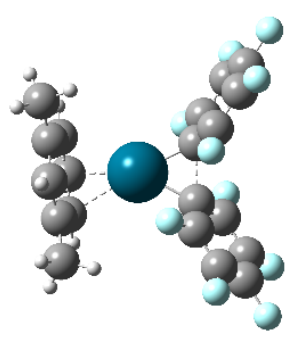

Number of imaginary frequencies $=1\left(i 253.33 \mathrm{~cm}^{-1}\right)$

$E_{\text {total }}=-1894.46039993 \mathrm{a} \cdot \mathrm{u}$

$\mathrm{G}_{\text {correction }}=0.190924 \mathrm{a} . \mathrm{u}$

\section{Cartesian coordinates:}

\begin{tabular}{|c|c|c|c|}
\hline $\mathrm{Pd}$ & -0.89550700 & -0.69278100 & -0.36230700 \\
\hline $\mathrm{C}$ & 1.13227600 & -0.42579500 & -0.09982100 \\
\hline C & 2.11395500 & -0.27314400 & -1.09747800 \\
\hline C & 1.52377900 & -1.22436600 & 0.99967600 \\
\hline $\mathrm{C}$ & 3.38430700 & -0.82717000 & -1.0036640 \\
\hline $\mathrm{C}$ & 2.78705800 & -1.78934200 & 1.1105940 \\
\hline $\mathrm{C}$ & 3.73231600 & -1.58435700 & 0.1099910 \\
\hline C & 0.08865000 & 1.07200700 & 0.0572650 \\
\hline C & 0.24066300 & 1.68527400 & 1.31521600 \\
\hline C & -0.16014500 & 1.96511500 & -1.01049900 \\
\hline C & 0.19229700 & 3.06153200 & 1.5007470 \\
\hline $\mathrm{C}$ & -0.21244200 & 3.34228700 & -0.8463410 \\
\hline $\mathrm{C}$ & -0.02573600 & 3.90175600 & 0.4146150 \\
\hline $\mathrm{F}$ & 1.86527600 & 0.46469700 & -2.1914600 \\
\hline $\mathrm{F}$ & 0.64425700 & -1.55401400 & 1.9673920 \\
\hline $\mathrm{F}$ & 3.09059500 & -2.56826500 & 2.1582850 \\
\hline $\mathrm{F}$ & 4.95037400 & -2.12523200 & 0.2072740 \\
\hline $\mathrm{F}$ & 4.27982700 & -0.62972100 & -1.9808260 \\
\hline $\mathrm{F}$ & 0.48228400 & 0.94560700 & 2.4098910 \\
\hline $\mathrm{F}$ & -0.44851200 & 1.50874900 & -2.2465160 \\
\hline $\mathrm{F}$ & -0.48713000 & 4.13875500 & -1.8889180 \\
\hline $\mathrm{F}$ & 0.36313800 & 3.58358100 & 2.7230400 \\
\hline $\mathrm{F}$ & -0.07799900 & 5.22644000 & 0.5824680 \\
\hline $\mathrm{C}$ & -2.36863700 & -3.28048200 & 0.58367300 \\
\hline C & -2.11358500 & -2.95842400 & -0.75717500 \\
\hline $\mathrm{C}$ & -2.68376900 & -1.79555300 & -1.3360650 \\
\hline $\mathrm{C}$ & -3.42827800 & -0.93502100 & -0.4885210 \\
\hline $\mathrm{C}$ & -3.67055300 & -1.25097200 & 0.86309700 \\
\hline C & -3.15732000 & -2.44904600 & 1.37881600 \\
\hline $\mathrm{H}$ & -1.53880800 & -3.63541800 & -1.3812270 \\
\hline $\mathrm{H}$ & -3.35072100 & -2.71277000 & 2.4138260 \\
\hline $\mathrm{H}$ & -3.87932500 & -0.04316400 & -0.91483500 \\
\hline $\mathrm{C}$ & -4.44270000 & -0.29880500 & 1.7385890 \\
\hline $\mathrm{H}$ & -3.76572900 & 0.43342800 & 2.19477000 \\
\hline $\mathrm{H}$ & -5.18983800 & 0.25750100 & 1.16576900 \\
\hline $\mathrm{H}$ & -4.94959400 & -0.82734400 & 2.5500310 \\
\hline $\mathrm{C}$ & -2.63897300 & -1.56698700 & -2.8296350 \\
\hline
\end{tabular}




$\begin{array}{llrr}\mathrm{H} & -3.48273000 & -2.07462000 & -3.31252200 \\ \mathrm{H} & -2.70266600 & -0.50378700 & -3.07207100 \\ \mathrm{H} & -1.71728700 & -1.96268000 & -3.26311500 \\ \mathrm{H} & -1.94794800 & -4.18965400 & 1.00133300\end{array}$

\section{4) Intermediate-31}

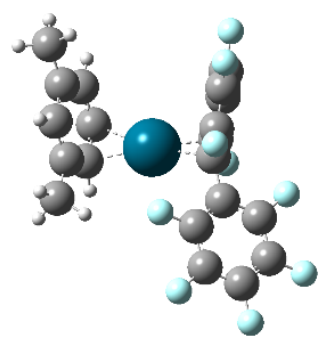

Number of imaginary frequencies $=0$

$E_{\text {total }}=-1894.50440132 \mathrm{a} . \mathrm{u}$

$\mathrm{G}_{\text {correction }}=0.190946 \mathrm{a} . \mathrm{u}$

\section{Cartesian coordinates:}

\begin{tabular}{|c|c|c|c|}
\hline $\mathrm{Pd}$ & -0.93972700 & -0.44953300 & -0.67713400 \\
\hline $\mathrm{C}$ & 1.92602200 & 0.24137800 & 0.17167000 \\
\hline C & 2.99842000 & 1.00636600 & -0.29597600 \\
\hline C & 2.20628200 & -1.06531500 & 0.57825400 \\
\hline C & 4.28514300 & 0.48636200 & -0.3973520 \\
\hline $\mathrm{C}$ & 3.48444100 & -1.60490000 & 0.4919220 \\
\hline C & 4.52866900 & -0.82488200 & 0.00104800 \\
\hline $\mathrm{C}$ & 0.56156900 & 0.82728100 & 0.26172700 \\
\hline C & 0.05156300 & 1.18460000 & 1.54812600 \\
\hline C & -0.05060500 & 1.46268700 & -0.8770490 \\
\hline $\mathrm{C}$ & -0.94876400 & 2.11327700 & 1.7087390 \\
\hline C & -1.06384700 & 2.44618400 & -0.6834490 \\
\hline C & -1.51568700 & 2.74311400 & 0.5806310 \\
\hline $\mathrm{F}$ & 2.80413200 & 2.28385900 & -0.6495700 \\
\hline $\mathrm{F}$ & 1.22600200 & -1.85316700 & 1.0487270 \\
\hline $\mathrm{F}$ & 3.71539900 & -2.86571500 & 0.8751600 \\
\hline $\mathrm{F}$ & 5.75999200 & -1.33322900 & -0.0837530 \\
\hline $\mathrm{F}$ & 5.28816800 & 1.24302500 & -0.8549190 \\
\hline $\mathrm{F}$ & 0.60580300 & 0.62185300 & 2.6339110 \\
\hline $\mathrm{F}$ & 0.62924300 & 1.53623500 & -2.0543220 \\
\hline $\mathrm{F}$ & -1.58705200 & 3.05584300 & -1.7552340 \\
\hline $\mathrm{F}$ & -1.43669500 & 2.39908000 & 2.9248970 \\
\hline F & -2.52992400 & 3.59880100 & 0.7582230 \\
\hline $\mathrm{C}$ & -2.24166100 & -2.70921200 & -0.0019190 \\
\hline $\mathrm{C}$ & -2.02583600 & -2.31058100 & -1.3475930 \\
\hline $\mathrm{C}$ & -2.90955100 & -1.38622000 & -1.9564250 \\
\hline C & -4.00463600 & -0.88412100 & -1.2402310 \\
\hline $\mathrm{C}$ & -4.23661600 & -1.27772500 & 0.0829210 \\
\hline C & -3.34450000 & -2.17904900 & 0.6818490 \\
\hline $\mathrm{H}$ & -2.77289300 & -1.11983700 & -2.9992720 \\
\hline $\mathrm{H}$ & -3.51683900 & -2.48745400 & 1.7102530 \\
\hline $\mathrm{H}$ & -1.26987100 & -2.81574500 & -1.9433140 \\
\hline $\mathrm{H}$ & -4.67932400 & -0.18098500 & -1.7196120 \\
\hline $\mathrm{C}$ & -1.36642700 & -3.75861400 & 0.6366330 \\
\hline
\end{tabular}




$\begin{array}{llll}\mathrm{H} & -0.35989800 & -3.74985300 & 0.21454000 \\ \mathrm{H} & -1.28379300 & -3.59995500 & 1.71494000 \\ \mathrm{H} & -1.79081900 & -4.75786800 & 0.48003400 \\ \mathrm{C} & -5.38773800 & -0.70292200 & 0.86819600 \\ \mathrm{H} & -5.75952500 & -1.41312300 & 1.61212600 \\ \mathrm{H} & -5.07490700 & 0.20061900 & 1.40617200 \\ \mathrm{H} & -6.21773900 & -0.42277300 & 0.21387800\end{array}$

\section{5) $\left[\mathrm{Pd}\left(\mathrm{C}_{6} \mathrm{H}_{4}\left(\mathrm{CH}_{3}\right)_{2}\right)_{2}\right](\mathbf{3 2})$}

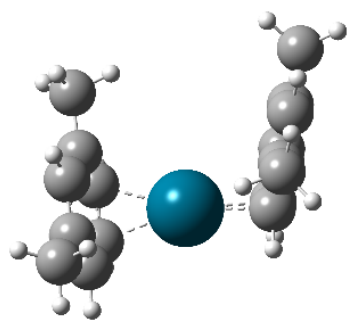

Number of imaginary frequencies $=0$

$\mathrm{E}_{\text {total }}=-749.82250306 \mathrm{a} . \mathrm{u}$

$\mathrm{G}_{\text {correction }}=0.262171 \mathrm{a} . \mathrm{u}$

\section{Cartesian coordinates:}

$\begin{array}{lr}\text { Pd } & -0.00032900 \\ \mathrm{C} & -1.20776100 \\ \mathrm{C} & -1.37827800 \\ \mathrm{C} & -1.98836500 \\ \mathrm{C} & -2.33768600 \\ \mathrm{C} & -2.93158600 \\ \mathrm{C} & -3.11841700 \\ \mathrm{C} & 3.11862200 \\ \mathrm{C} & 2.93177200 \\ \mathrm{C} & 1.98853000 \\ \mathrm{C} & 1.20819700 \\ \mathrm{C} & 1.37861200 \\ \mathrm{C} & 2.33786500 \\ \mathrm{H} & 3.53702000 \\ \mathrm{H} & 2.48841100 \\ \mathrm{H} & 2.00280500 \\ \mathrm{H} & 0.60894800 \\ \mathrm{C} & 0.50891700 \\ \mathrm{H} & 0.90753600 \\ \mathrm{H} & -0.50124400 \\ \mathrm{H} & 0.41546900 \\ \mathrm{H} & -0.60800200 \\ \mathrm{H} & -2.00271800 \\ \mathrm{H} & -3.53697100 \\ \mathrm{H} & -2.48830400 \\ \mathrm{C} & -0.50857800 \\ \mathrm{H} & -0.90725200 \\ \mathrm{H} & 0.50158900 \\ \mathrm{H} & -0.41513400 \\ \mathrm{C} & 4.11870300 \\ \mathrm{H} & 3.61494300 \\ & \end{array}$

$\begin{array}{rr}-1.19308200 & -0.00040100 \\ -0.33059700 & -1.70579300 \\ 1.04564900 & -1.38243800 \\ -1.31423300 & -1.04901600 \\ 1.39667800 & -0.44234100 \\ -0.91148500 & -0.06651300 \\ 0.43165900 & 0.23458100 \\ 0.43180200 & -0.23440300 \\ -0.91179700 & 0.06462200 \\ -1.31605100 & 1.04646200 \\ -0.33343900 & 1.70507300 \\ 1.04329200 & 1.38370700 \\ 1.39576900 & 0.44400600 \\ -1.66616100 & -0.42995400 \\ 2.44857300 & 0.21442000 \\ -2.33921800 & 1.41319500 \\ -0.60183200 & 2.57143600 \\ 2.07753800 & 2.04923600 \\ 3.08587000 & 1.90840600 \\ 2.05173800 & 1.62385900 \\ 1.89137500 & 3.12463300 \\ -0.59774800 & -2.57218600 \\ -2.33679200 & -1.41740400 \\ -1.66655900 & 0.42680600 \\ 2.44912300 & -0.21116700 \\ 2.08092200 & -2.04635900 \\ 3.08902500 & -1.90403900 \\ 2.05457900 & -1.62103400 \\ 1.89636800 & -3.12203000 \\ 0.87227200 & -1.27418400 \\ 1.28790400 & -2.15545600\end{array}$


4.78071200

4.74003300

$-4.11849100$

$-3.61474300$

$-4.78032700$

$-4.74000900$
1.65403000

0.03768900

0.87049200

1.28448700

1.65305000

0.03544000
$-0.88554200$

$-1.60969200$

1.27507000

2.15711800

0.88774500

1.60906300

56) $\left(\mathrm{C}_{6} \mathrm{~F}_{5}\right)_{2}$

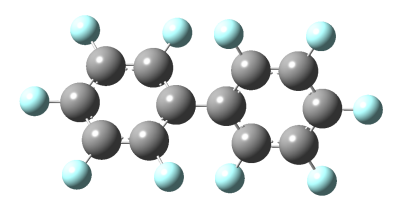

Number of imaginary frequencies $=0$

$E_{\text {total }}=-1455.61603218$ a.u

$\mathrm{G}_{\text {correction }}=0.053198 \mathrm{a} . \mathrm{u}$

\section{Cartesian coordinates:}

$\begin{array}{lrrr}C & 0.73804600 & 0.00001000 & -0.00000100 \\ C & 1.46467600 & -1.06542000 & -0.54026700 \\ C & 1.46468400 & 1.06543100 & 0.54026600 \\ C & 2.85505700 & -1.07801900 & -0.53991700 \\ C & 2.85506900 & 1.07801700 & 0.53991700 \\ C & 3.55158500 & -0.00000200 & -0.00000100 \\ C & -0.73804700 & 0.00001100 & -0.00000200 \\ C & -1.46468300 & 1.06542100 & -0.54027800 \\ C & -1.46467600 & -1.06541900 & 0.54027700 \\ C & -2.85507300 & 1.07801300 & -0.53993100 \\ C & -2.85505400 & -1.07801500 & 0.53992600 \\ C & -3.55158300 & 0.00000000 & -0.00000300 \\ \text { F } & 0.82128000 & -2.10403000 & -1.08719900 \\ \text { F } & 0.82130500 & 2.10403800 & 1.08721800 \\ \text { F } & 3.52355400 & 2.10941900 & 1.06529400 \\ \text { F } & 4.88547200 & -0.00001400 & 0.00000000 \\ \text { F } & 3.52354100 & -2.10942800 & -1.06528200 \\ \text { F } & -0.82130800 & 2.10403000 & -1.08723700 \\ \text { F } & -0.82127500 & -2.10401600 & 1.08722300 \\ \text { F } & -3.52355000 & -2.10941000 & 1.06530300 \\ \text { F } & -3.52354900 & 2.10941400 & -1.06531500 \\ \text { F } & -4.88547100 & -0.00002200 & 0.00000600 \\ \text { C } & & \end{array}$

57) $\left[\mathrm{Pd}\left(\mathrm{C}_{6} \mathrm{~F}_{5}\right)_{2}\right](\mathbf{3 3})$

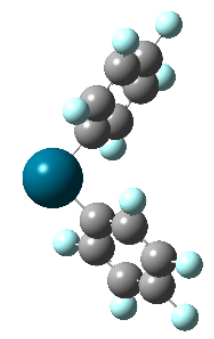


Number of imaginary frequencies $=0$

$E_{\text {total }}=-1583.52136677$ a.u

$\mathrm{G}_{\text {correction }}=0.046162 \mathrm{a} . \mathrm{u}$

\section{Cartesian coordinates:}

Pd
C
C
C
C
C
C
C
C
C
C
C
C
F
F
F
F
F
F
F
F
F
F

$-0.00510700$

$-1.34357400$

$-2.31600500$

$-1.38188100$

$-3.31572500$

$-2.37032100$

$-3.33885700$

1.34013800

2.29147000

1. 40379100

3.29728400

2.39908600

3. 34702500

$-2.32203900$

$-0.45829000$

$-2.39915900$

$-4.29597000$

$-4.25111300$

2.26681700

0.49913400

2.45477700

4.21309300

4.31056600
2.04223500

0.60834900

0.50470900

$-0.31274300$

$-0.46246100$

$-1.29124400$

$-1.36516200$

0.63821000

0.41012400

$-0.13909300$

$-0.53945900$

$-1.09677800$

$-1.29654200$

1.35927100

$-0.28463100$

$-2.15924700$

$-2.29588200$

$-0.53390000$

1.12289600

0.01386800

$-1.82482900$

$-0.73264100$

$-2.20826700$
0.11259500

0.23101000

$-0.76039000$

1.27365200

$-0.71029100$

1. 34862700

0.35043400

$-0.16428700$

0.82621200

$-1.31658500$

0.67275200

$-1.49671700$

$-0.49578800$

$-1.80419600$

2. 25249300

2. 36824000

0.41495600

$-1.66610700$

1.97081800

$-2.30172200$

$-2.61961600$

1.63074300

$-0.66017900$

\section{8) $\mathbf{C}_{6} \mathbf{H}_{4}\left(\mathrm{CH}_{3}\right)_{2}$}

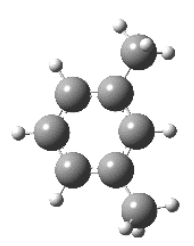

Number of imaginary frequencies $=0$

$E_{\text {total }}=-310.92982518 \mathrm{a} . \mathrm{u}$

$\mathrm{G}_{\text {correction }}=0.121323 \mathrm{a} . \mathrm{u}$

\section{Cartesian coordinates:}

$\mathrm{C}$
$\mathrm{C}$
$\mathrm{C}$
$\mathrm{C}$
$\mathrm{C}$
$\mathrm{C}$
$\mathrm{H}$
$\mathrm{H}$
$\mathrm{H}$
$\mathrm{H}$
$\mathrm{C}$

$$
\begin{array}{r}
-0.00613700 \\
1.20760200 \\
1.22675000 \\
0.00319200 \\
-1.22020900 \\
-1.21093200 \\
-0.00867300 \\
2.14490400 \\
0.00682400 \\
-2.15265000 \\
-2.52536800
\end{array}
$$

1.82296300

1.13174600

$-0.26532300$

$-0.94965100$

$-0.27617700$

1. 12633100

2.90914800

1.68143700

$-2.03761400$

1.66906800

$-1.03349100$
0.00135500

$-0.00547100$

$-0.00705000$

$-0.00543500$

0.00125000

0.00520900

0.00142000

$-0.01039100$

$-0.01053600$

0.00877400

0.00027400 


$\begin{array}{lrrr}\mathrm{H} & -3.13363600 & -0.77786500 & 0.87508600 \\ \mathrm{H} & -3.12291900 & -0.79425100 & -0.88661300 \\ \mathrm{H} & -2.35971500 & -2.11394600 & 0.01103600 \\ \mathrm{C} & 2.52812800 & -1.02893900 & 0.00549100 \\ \mathrm{H} & 2.70134900 & -1.50056800 & 0.98010600 \\ \mathrm{H} & 2.52890600 & -1.82827400 & -0.74282600 \\ \mathrm{H} & 3.37745300 & -0.37188300 & -0.19979400\end{array}$

\section{9) $\mathrm{TS}(33-34)$}

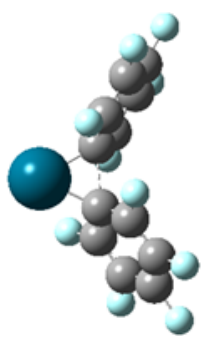

Number of imaginary frequencies $=1\left(i 267.04 \mathrm{~cm}^{-1}\right)$

$\mathrm{E}_{\text {total }}=-1583.49251934 \mathrm{a} . \mathrm{u}$

$\mathrm{G}_{\text {correction }}=0.048598 \mathrm{a} . \mathrm{u}$

Cartesian coordinates:

$\begin{array}{lrrr}\text { Pd } & -0.00006600 & 0.00028800 & 2.15992200 \\ \text { C } & -0.91805500 & -0.12230500 & 0.32843600 \\ \text { C } & -1.84077500 & 0.94448900 & 0.23272200 \\ \text { C } & -1.28207100 & -1.28718500 & -0.37445400 \\ \text { C } & -3.00355900 & 0.87074000 & -0.52263100 \\ \text { C } & -2.43995000 & -1.38002800 & -1.13685900 \\ \text { C } & -3.30577200 & -0.29446100 & -1.22135300 \\ \text { C } & 0.91809700 & 0.12240500 & 0.32845900 \\ \text { C } & 1.84080800 & -0.94442200 & 0.23303200 \\ \text { C } & 1.28213200 & 1.28709900 & -0.37472700 \\ \text { C } & 3.00359400 & -0.87087600 & -0.52234100 \\ \text { C } & 2.44000200 & 1.37973200 & -1.13717100 \\ \text { C } & 3.30581600 & 0.29413700 & -1.22137200 \\ \text { F } & -1.66883200 & 2.07688900 & 0.94397700 \\ \text { F } & -0.48092400 & -2.36349700 & -0.37244800 \\ \text { F } & -2.72265000 & -2.50945700 & -1.79832300 \\ \text { F } & -4.42413300 & -0.37704100 & -1.94570900 \\ \text { F } & -3.85885800 & 1.90077200 & -0.55113500 \\ \text { F } & 1.66882200 & -2.07666200 & 0.94452100 \\ \text { F } & 0.48098300 & 2.36341000 & -0.37301700 \\ \text { F } & 2.72269300 & 2.50897800 & -1.79895200 \\ \text { F } & 3.85888300 & -1.90092400 & -0.55058800 \\ \text { F } & 4.42417600 & 0.37650900 & -1.94575400\end{array}$

60) Intermediate-34 


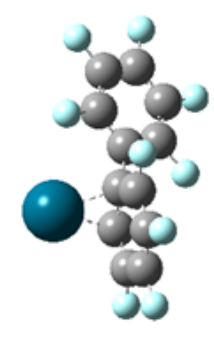

Number of imaginary frequencies $=0$

$\mathrm{E}_{\text {total }}=-1583.53502571 \mathrm{a} . \mathrm{u}$

$\mathrm{G}_{\text {correction }}=0.049147 \mathrm{a} . \mathrm{u}$

Cartesian coordinates:

\begin{tabular}{|c|c|c|c|}
\hline $\mathrm{Pd}$ & 1.19823400 & -1.49600600 & -1.12332600 \\
\hline $\mathrm{C}$ & -1.05083800 & 0.13995400 & 0.15882000 \\
\hline $\mathrm{C}$ & -1.87203100 & -0.97529200 & 0.33605200 \\
\hline $\mathrm{C}$ & -1.67957200 & 1.37564600 & -0.01603600 \\
\hline C & -3.25862100 & -0.88063200 & 0.30893900 \\
\hline $\mathrm{C}$ & -3.06538400 & 1.49506900 & -0.05337100 \\
\hline $\mathrm{C}$ & -3.85697700 & 0.36208800 & 0.11382000 \\
\hline $\mathrm{C}$ & 0.43365500 & 0.04387700 & 0.20118300 \\
\hline $\mathrm{C}$ & 1.24593000 & 0.58756200 & -0.86516100 \\
\hline C & 1.06579800 & -0.11536800 & 1.47291200 \\
\hline C & 2.58452400 & 1.00430700 & -0.59554000 \\
\hline C & 2.36714400 & 0.26714100 & 1.69855200 \\
\hline $\mathrm{C}$ & 3.13302100 & 0.82579200 & 0.65327000 \\
\hline F & -1.32737500 & -2.19208500 & 0.50494000 \\
\hline $\mathrm{F}$ & -0.94330600 & 2.48760500 & -0.14126300 \\
\hline $\mathrm{F}$ & -3.63886000 & 2.68967900 & -0.22899700 \\
\hline $\mathrm{F}$ & -5.18701300 & 0.46624500 & 0.08677800 \\
\hline $\mathrm{F}$ & -4.01708400 & -1.97076300 & 0.46460300 \\
\hline $\mathrm{F}$ & 0.64145200 & 1.20265600 & -1.91936500 \\
\hline $\mathrm{F}$ & 0.33896400 & -0.60286900 & 2.49072100 \\
\hline $\mathrm{F}$ & 2.94248900 & 0.08209900 & 2.89458600 \\
\hline $\mathrm{F}$ & 3.29374700 & 1.57697000 & -1.57632000 \\
\hline $\mathrm{F}$ & 4.40824700 & 1.15328700 & 0.89013400 \\
\hline
\end{tabular}

61) $\left[\mathrm{Pd}\left(\mathrm{C}_{6} \mathrm{~F}_{6}\right)\left(\mathrm{C}_{6} \mathrm{~F}_{5}\right)_{2}\right](\mathbf{3 5})$

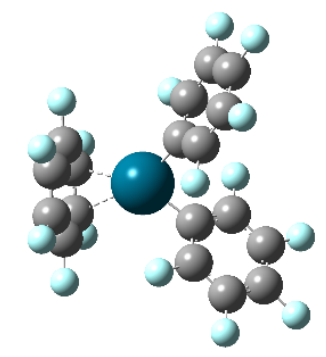

Number of imaginary frequencies $=0$

$E_{\text {total }}=-2411.16082538 \mathrm{a} . \mathrm{u}$

$\mathrm{G}_{\text {correction }}=0.080266 \mathrm{a} . \mathrm{u}$

Cartesian coordinates: 


\begin{tabular}{|c|c|c|c|}
\hline $\mathrm{Pd}$ & -0.72044900 & 0.00002100 & 0.00000100 \\
\hline $\mathrm{C}$ & 0.71573800 & -1.33618800 & -0.20886400 \\
\hline $\mathrm{C}$ & 1.62354800 & -1.34809400 & -1.26000600 \\
\hline $\mathrm{C}$ & 0.79745400 & -2.36128500 & 0.72656400 \\
\hline $\mathrm{C}$ & 2.57727200 & -2.35534800 & -1.39147500 \\
\hline $\mathrm{C}$ & 1.73679600 & -3.38212400 & 0.6236000 \\
\hline C & 2.63304000 & -3.37394000 & -0.44326100 \\
\hline $\mathrm{C}$ & 0.71578400 & 1.33618100 & 0.20886900 \\
\hline $\mathrm{C}$ & 1.62360900 & 1.34804700 & 1.2599980 \\
\hline C & 0.79752100 & 2.36128600 & -0.72654900 \\
\hline C & 2.57736700 & 2.35526800 & 1.3914640 \\
\hline C & 1.73689900 & 3.38209300 & -0.6235890 \\
\hline $\mathrm{C}$ & 2.63315600 & 3.37386800 & 0.4432600 \\
\hline $\mathrm{F}$ & 1.60465000 & -0.37994700 & -2.1970850 \\
\hline $\mathrm{F}$ & -0.05839000 & -2.39127200 & 1.7727570 \\
\hline $\mathrm{F}$ & 1.79034200 & -4.36525700 & 1.5319410 \\
\hline $\mathrm{F}$ & 3.54003700 & -4.34964100 & -0.5615930 \\
\hline $\mathrm{F}$ & 3.43653800 & -2.35684800 & -2.419312 \\
\hline $\mathrm{F}$ & 1.60469200 & 0.37989200 & 2.197069 \\
\hline $\mathrm{F}$ & -0.05833800 & 2.39131200 & -1.772730 \\
\hline $\mathrm{F}$ & 1.79046300 & 4.36523300 & -1.531921 \\
\hline $\mathrm{F}$ & 3.43664800 & 2.35673100 & 2.4192900 \\
\hline $\mathrm{F}$ & 3.54018700 & 4.34953700 & 0.5615900 \\
\hline $\mathrm{C}$ & -3.00747600 & -1.38418000 & -0.18490000 \\
\hline $\mathrm{C}$ & -3.04539500 & -0.53089300 & -1.30312800 \\
\hline C & -3.04935400 & 0.85607500 & -1.1182470 \\
\hline C & -3.00749400 & 1.38424300 & 0.18489700 \\
\hline C & -3.04542600 & 0.53095600 & 1.30312400 \\
\hline C & -3.04936700 & -0.85601200 & 1.11824300 \\
\hline $\mathrm{F}$ & -2.99360400 & 2.69978400 & 0.36223200 \\
\hline $\mathrm{F}$ & -3.06080500 & 1.04492700 & 2.5256020 \\
\hline $\mathrm{F}$ & -3.07247100 & -1.67104000 & 2.1626670 \\
\hline $\mathrm{F}$ & -2.99356900 & -2.69972100 & -0.3622350 \\
\hline $\mathrm{F}$ & -3.06075300 & -1.04486500 & -2.5256060 \\
\hline $\mathrm{F}$ & -3.07244700 & 1.67110300 & -2.1626710 \\
\hline
\end{tabular}

\section{2) $\mathrm{TS}(35-36)$}

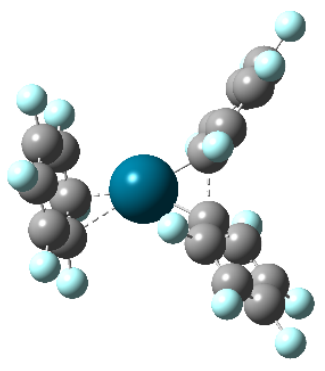

Number of imaginary frequencies $=1\left(i 288.94 \mathrm{~cm}^{-1}\right)$

$\mathrm{E}_{\text {total }}=-2411.13920377 \mathrm{a} . \mathrm{u}$

$\mathrm{G}_{\text {correction }}=0.085283 \mathrm{a} . \mathrm{u}$

\section{Cartesian coordinates:}

$\begin{array}{lrrr}\mathrm{Pd} & 0.10956600 & -1.29746700 & -0.32903600 \\ \mathrm{C} & 0.14529700 & 0.72459600 & 0.03750900\end{array}$




\begin{tabular}{|c|c|c|c|}
\hline C & 0.03681600 & 1.32585300 & 1.30266400 \\
\hline C & -0.54036000 & 1.38301900 & -1.00341900 \\
\hline C & -0.68531500 & 2.49341300 & 1.52134900 \\
\hline $\mathrm{C}$ & -1.27178200 & 2.54763600 & -0.80446900 \\
\hline $\mathrm{C}$ & -1.33945900 & 3.11695500 & 0.46335800 \\
\hline C & 1.81676700 & -0.13691200 & -0.20064200 \\
\hline C & 2.54442600 & 0.45560200 & -1.24706500 \\
\hline C & 2.58893400 & -0.62238000 & 0.87639900 \\
\hline $\mathrm{C}$ & 3.92746500 & 0.58825200 & -1.22022000 \\
\hline C & 3.97167100 & -0.50105900 & 0.92230000 \\
\hline $\mathrm{C}$ & 4.64791600 & 0.11489600 & -0.12759800 \\
\hline $\mathrm{F}$ & 0.64226800 & 0.78155700 & 2.36915700 \\
\hline $\mathrm{F}$ & -0.62298300 & 0.83529800 & -2.23295800 \\
\hline $\mathrm{F}$ & -1.95310900 & 3.09450200 & -1.81697900 \\
\hline $\mathrm{F}$ & -2.04682600 & 4.22951800 & 0.66851300 \\
\hline $\mathrm{F}$ & -0.75611300 & 3.01879800 & 2.74941400 \\
\hline $\mathrm{F}$ & 1.91381300 & 0.96116400 & -2.31640600 \\
\hline $\mathrm{F}$ & 2.01679200 & -1.30470500 & 1.88762600 \\
\hline $\mathrm{F}$ & 4.66398700 & -1.00720100 & 1.94929900 \\
\hline $\mathrm{F}$ & 4.56972500 & 1.17289100 & -2.23806800 \\
\hline $\mathrm{F}$ & 5.97630300 & 0.23051000 & -0.09627700 \\
\hline $\mathrm{C}$ & -2.54086200 & -0.99535300 & 1.40798100 \\
\hline C & -1.92238700 & -2.16623800 & 0.95354500 \\
\hline C & -1.90376300 & -2.46454500 & -0.42822300 \\
\hline C & -2.53694200 & -1.58491700 & -1.33457800 \\
\hline C & -3.13615300 & -0.42171900 & -0.86943900 \\
\hline C & -3.13438100 & -0.12900300 & 0.49827000 \\
\hline $\mathrm{F}$ & -2.56751300 & -1.89319100 & -2.62871400 \\
\hline $\mathrm{F}$ & -3.66287500 & 0.45257400 & -1.72666400 \\
\hline $\mathrm{F}$ & -3.64897700 & 1.02638100 & 0.91971600 \\
\hline $\mathrm{F}$ & -2.50814500 & -0.69004700 & 2.70479700 \\
\hline F & -1.46431200 & -3.06217700 & 1.82924000 \\
\hline $\mathrm{F}$ & -1.55730000 & -3.69976900 & -0.83843600 \\
\hline
\end{tabular}

\section{3) Intermediate-36}

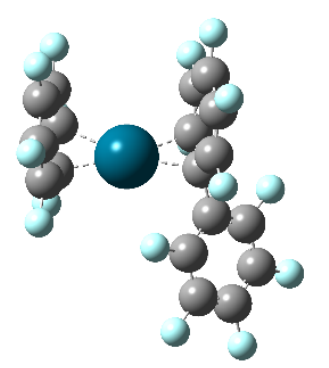

Number of imaginary frequencies $=0$

$\mathrm{E}_{\text {total }}=-2411.18552022 \mathrm{a} . \mathrm{u}$

$\mathrm{G}_{\text {correction }}=0.085107 \mathrm{a} . \mathrm{u}$

\section{Cartesian coordinates:}

$\begin{array}{lrrr}\mathrm{Pd} & -0.49783900 & -0.65868800 & -0.46142000 \\ \mathrm{C} & 2.40484800 & 0.22943000 & 0.22825000 \\ \mathrm{C} & 3.53668900 & 0.99760400 & -0.06157200 \\ \mathrm{C} & 2.60179100 & -1.13218700 & 0.46245900\end{array}$


4.80697500

3.85912600

4.96784700

1.06476300

0.36288400

0.60069800

$-0.69613100$

$-0.44687600$

$-1.09514200$

3. 41061800

1.54129900

4.00866500

6.18006200

5.86960200

0.78374300

1. 25489700

$-0.85813300$

$-1.34933900$

$-2.13350900$

$-2.83758600$

$-2.15645800$

$-2.34821500$

$-3.21727900$

$-3.81983500$

$-3.62995000$

$-3.40448400$

$-4.54378800$

$-4.19025500$

$-2.69420000$

$-1.81235700$

$-2.15707900$
0.43386500

$-1.71948900$

$-0.92844700$

0.86730400

0.94491600

1.71295200

1.82689300

2.61512500

2.66841100

2. 31242200

$-1.92553600$

$-3.02677400$

$-1.47681900$

1.19167800

0.21055100

1. 73841600

3. 38652600

1. 85138200

3. 48168500

$-2.32941700$

$-1.83470800$

$-0.45877400$

0.35584200

$-0.14836200$

$-1.49917000$

1.63111200

0.64448300

$-1.93703600$

$-3.61892800$

$-2.73739600$

$-0.09366200$

$$
\begin{array}{r}
-0.12590200 \\
0.41246100 \\
0.11709400 \\
0.30746400 \\
1.53944200 \\
-0.73392600 \\
1.72972900 \\
-0.54046100 \\
0.68996900 \\
-0.27561000 \\
0.71792700 \\
0.63259500 \\
0.06268800 \\
-0.40420500 \\
2.57491400 \\
-1.89960400 \\
-1.54736900 \\
2.89385800 \\
0.86074800 \\
0.02144900 \\
-1.13211500 \\
-1.50565200 \\
-0.72136700 \\
0.40615400 \\
0.77965900 \\
-1.08823400 \\
1.20380200 \\
1.91334600 \\
0.34369900 \\
-2.08324600 \\
-2.79569600
\end{array}
$$

\section{4) $\left[\mathrm{Pd}\left(\mathrm{C}_{6} \mathrm{~F}_{6}\right)_{2}\right](37)$}

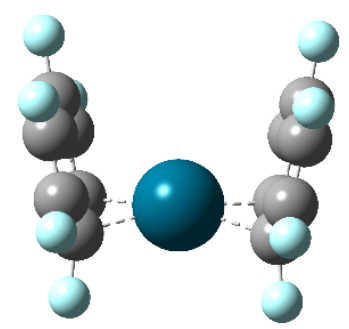

Number of imaginary frequencies $=0$

$\mathrm{E}_{\text {total }}=-1783.19104704 \mathrm{a} . \mathrm{u}$

$\mathrm{G}_{\text {correction }}=0.045590 \mathrm{a} . \mathrm{u}$

\section{Cartesian coordinates:}

$\begin{array}{ll}\mathrm{Pd} & 0.00002500 \\ \mathrm{C} & 2.10877000 \\ \mathrm{C} & 2.34468100 \\ \mathrm{C} & 2.09913800 \\ \mathrm{C} & 2.55416200 \\ \mathrm{C} & 2.32516800 \\ \mathrm{C} & 2.54419100\end{array}$

$-0.73478100$

$-1.02791100$

0.16773200

$-0.99176600$

1.36159000

0.23950100

1.39734200
$-0.01062700$

0.68265100

1.40331100

$-0.74136200$

0.72998500

$-1.40297800$

$-0.67241200$ 
2.39445300

2.23463100

2.35641900

2.71675000

2.69730200

$-2.33696100$

$-2.10459200$

$-2.10275100$

$-2.33276300$

$-2.54830700$

$-2.55048700$

$-2.37132700$

$-2.70532500$

$-2.70979900$

$-2.37946900$

$-2.24390500$

$-2.24165700$

2.25163200
0.12429300

$-2.13355600$

0.26499500

2.49706600

2.56609900

0.17738600

$-1.02333800$

$-0.99730500$

0.22914800

1.39207900

1. 36632100

0.24503300

2. 55631700

2. 50657500

0.14352300

$-2.19446800$

$-2.14401800$

$-2.20349900$
2.73529200

$-1.43663900$

$-2.73597800$

1.41267400

$-1.29881400$

1.40991300

0.69649600

$-0.72779200$

$-1.39692000$

$-0.67342100$

0.72923500

$-2.72988200$

$-1.30722000$

1. 40471400

2. 74242700

1. 34018000

$-1.41420500$

1. 31729400

65) $\left(\mathrm{C}_{6} \mathrm{~F}_{5}\right)_{2}$

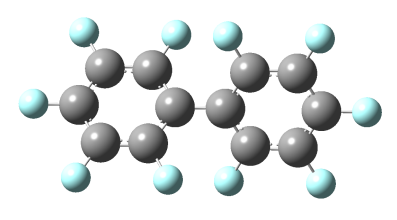

Number of imaginary frequencies $=0$

$$
E_{\text {total }}=-1455.61579449 \text { a.u }
$$

$\mathrm{G}_{\text {correction }}=0.053233 \mathrm{a} . \mathrm{u}$

\section{Cartesian coordinates:}

$\begin{array}{lr}\text { C } & 0.73804900 \\ C & 1.46461200 \\ C & 1.46462000 \\ C & 2.85506000 \\ C & 2.85507000 \\ C & 3.55159100 \\ C & -0.73804900 \\ C & -1.46462300 \\ C & -1.46460900 \\ C & -2.85507600 \\ C & -2.85505500 \\ C & -3.55159000 \\ \text { F } & 0.82109400 \\ \text { F } & 0.82111600 \\ \text { F } & 3.52352700 \\ \text { F } & 4.88537600 \\ \text { F } & 3.52351300 \\ \text { F } & -0.82112500 \\ \text { F } & -0.82108400 \\ \text { F } & -3.52351100 \\ \text { F } & -3.52353000 \\ \text { F } & -4.88537600 \\ & \end{array}$

$$
\begin{array}{r}
0.00001400 \\
-1.06542400 \\
1.06544100 \\
-1.07803400 \\
1.07803700 \\
0.00000000 \\
0.00001200 \\
1.06543000 \\
-1.06542400 \\
1.07802700 \\
-1.07803300 \\
-0.00000400 \\
-2.10371700 \\
2.10372600 \\
2.10922600 \\
-0.00001200 \\
-2.10923500 \\
2.10371800 \\
-2.10370400 \\
-2.10922300 \\
2.10921600 \\
-0.00002100
\end{array}
$$

$-0.00000700$

$-0.54046100$

0.54045500

$-0.54012700$

0.54012700

0.00000000

$-0.00000600$

$-0.54047500$

0.54046400

$-0.54014100$

0.54013500

0.00000000

$-1.08760800$

1.08763200

1.06565100

0.00000600

$-1.06563100$

$-1.08765700$

1.08762600

1.06565800

$-1.06566500$

0.00001100 
66) $\left[\mathrm{Pd}\left(\mathrm{C}_{6} \mathrm{~F}_{5}\right)_{2}\right](\mathbf{3 8})$

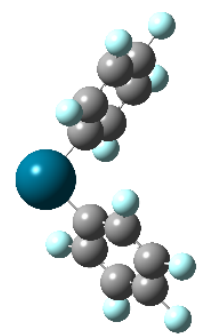

Number of imaginary frequencies $=0$

$\mathrm{E}_{\text {total }}=-1583.51933230 \mathrm{a} . \mathrm{u}$

$\mathrm{G}_{\text {correction }}=0.046096 \mathrm{a} . \mathrm{u}$

Cartesian coordinates:

\begin{tabular}{|c|c|c|c|}
\hline $\mathrm{Pd}$ & -0.00543300 & 2.03824700 & 0.11714600 \\
\hline C & -1.34585200 & 0.60646100 & 0.23251100 \\
\hline $\mathrm{C}$ & -2.31874500 & 0.50827700 & -0.75902800 \\
\hline C & -1.38321700 & -0.31824800 & 1.27209500 \\
\hline C & -3.31991200 & -0.45753900 & -0.71107000 \\
\hline C & -2.37323700 & -1.29541300 & 1.34431400 \\
\hline C & -3.34291600 & -1.36400800 & 0.34661200 \\
\hline C & 1.34218200 & 0.63751200 & -0.16296600 \\
\hline C & 2.29337900 & 0.40969800 & 0.82788000 \\
\hline $\mathrm{C}$ & 1.40584800 & -0.13747000 & -1.31687500 \\
\hline C & 3.30082600 & -0.53782400 & 0.67221700 \\
\hline C & 2.40305600 & -1.09291500 & -1.49868900 \\
\hline $\mathrm{C}$ & 3.35143200 & -1.29257500 & -0.49796800 \\
\hline $\mathrm{F}$ & -2.32363100 & 1.36685500 & -1.79898900 \\
\hline $\mathrm{F}$ & -0.45807100 & -0.29482800 & 2.24898500 \\
\hline $\mathrm{F}$ & -2.40240900 & -2.16718700 & 2.36027100 \\
\hline $\mathrm{F}$ & -4.30112500 & -2.29328700 & 0.40881300 \\
\hline $\mathrm{F}$ & -4.25637800 & -0.52413500 & -1.66572400 \\
\hline $\mathrm{F}$ & 2.26695200 & 1.12061400 & 1.97311900 \\
\hline $\mathrm{F}$ & 0.50027700 & 0.01574800 & -2.30049400 \\
\hline $\mathrm{F}$ & 2.46005000 & -1.81887900 & -2.62248700 \\
\hline $\mathrm{F}$ & 4.21712900 & -0.73124300 & 1.62923400 \\
\hline $\mathrm{F}$ & 4.31641000 & -2.20200300 & -0.66416300 \\
\hline
\end{tabular}

\section{7) $\mathbf{C}_{6} \mathbf{F}_{6}$}

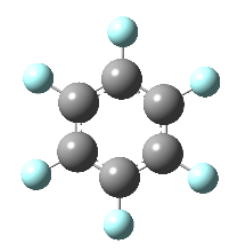

Number of imaginary frequencies $=0$

$E_{\text {total }}=-827.61889216 \mathrm{a} . \mathrm{u}$

$\mathrm{G}_{\text {correction }}=0.016070 \mathrm{a} . \mathrm{u}$

Cartesian coordinates: 


$\begin{array}{lrrr}C & -0.76235900 & 1.16565700 & -0.00002200 \\ C & 0.62825500 & 1.24305900 & -0.00001300 \\ C & 1.39040200 & 0.07739700 & -0.00001800 \\ C & 0.76233300 & -1.16567400 & -0.00001000 \\ C & -0.62822800 & -1.24307400 & -0.00002600 \\ C & -1.39040300 & -0.07736700 & -0.00000700 \\ F & -1.23134600 & -2.43502600 & 0.00000300 \\ F & 2.72411400 & 0.15158600 & 0.00001000 \\ F & 1.49407000 & -2.28324700 & 0.00001600 \\ F & 1.23126300 & 2.43506700 & 0.00001400 \\ F & -1.49399200 & 2.28329800 & 0.00000700 \\ F & -2.72410900 & -0.15167800 & 0.00001500\end{array}$

\section{8) TS(38-39)}

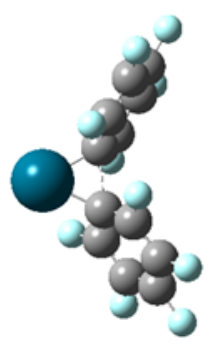

Number of imaginary frequencies $=1\left(i 269.85 \mathrm{~cm}^{-1}\right)$

$\mathrm{E}_{\text {total }}=-1583.49109841 \mathrm{a} . \mathrm{u}$

$\mathrm{G}_{\text {correction }}=0.048613 \mathrm{a} . \mathrm{u}$

\section{Cartesian coordinates:}

Pd
C
C
C
C
C
C
C
C
C
C
C
C
F
F
F
F
F
F
F
F
F
F

$$
\begin{array}{r}
-0.00003200 \\
-0.92164900 \\
-1.84380500 \\
-1.28224000 \\
-3.00506200 \\
-2.43861000 \\
-3.30480100 \\
0.92167100 \\
1.84382600 \\
1.28226300 \\
3.00509100 \\
2.43863000 \\
3.30482800 \\
-1.67261400 \\
-0.48017400 \\
-2.71941600 \\
-4.42140500 \\
-3.86061400 \\
1.67260800 \\
0.48018300 \\
2.71942000 \\
3.86064500 \\
4.42143700
\end{array}
$$

0.00000800

$-0.12305100$

0.94364300

$-1.28794100$

0.86971800

$-1.38087500$

$-0.29549200$

0.12305300

$-0.94364200$

1.28793800

$-0.86971000$

1.38087200

0.29549500

2.07512500

$-2.36319300$

$-2.50971100$

$-0.37831800$

1.89911100

$-2.07514600$

2.36317800

2.50969800

$-1.89910200$

0.37831400
2.16029600

0.33092600

0.23465300

$-0.37302000$

$-0.52326900$

$-1.13793500$

$-1.22325400$

0.33094900

0.23468400

$-0.37300500$

$-0.52322700$

$-1.13792200$

$-1.22322200$

0.94691200

$-0.36926100$

$-1.80069000$

$-1.94983400$

$-0.55335600$

0.94689500

$-0.36927200$

$-1.80070200$

$-0.55331600$

$-1.94979700$

69) Intermediate-39 


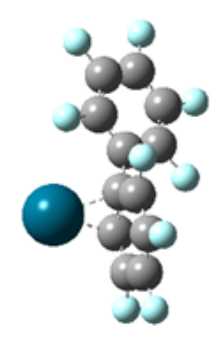

Number of imaginary frequencies $=0$

$\mathrm{E}_{\text {total }}=-1583.53391677$ a.u

$\mathrm{G}_{\text {correction }}=0.049136 \mathrm{a} . \mathrm{u}$

Cartesian coordinates:

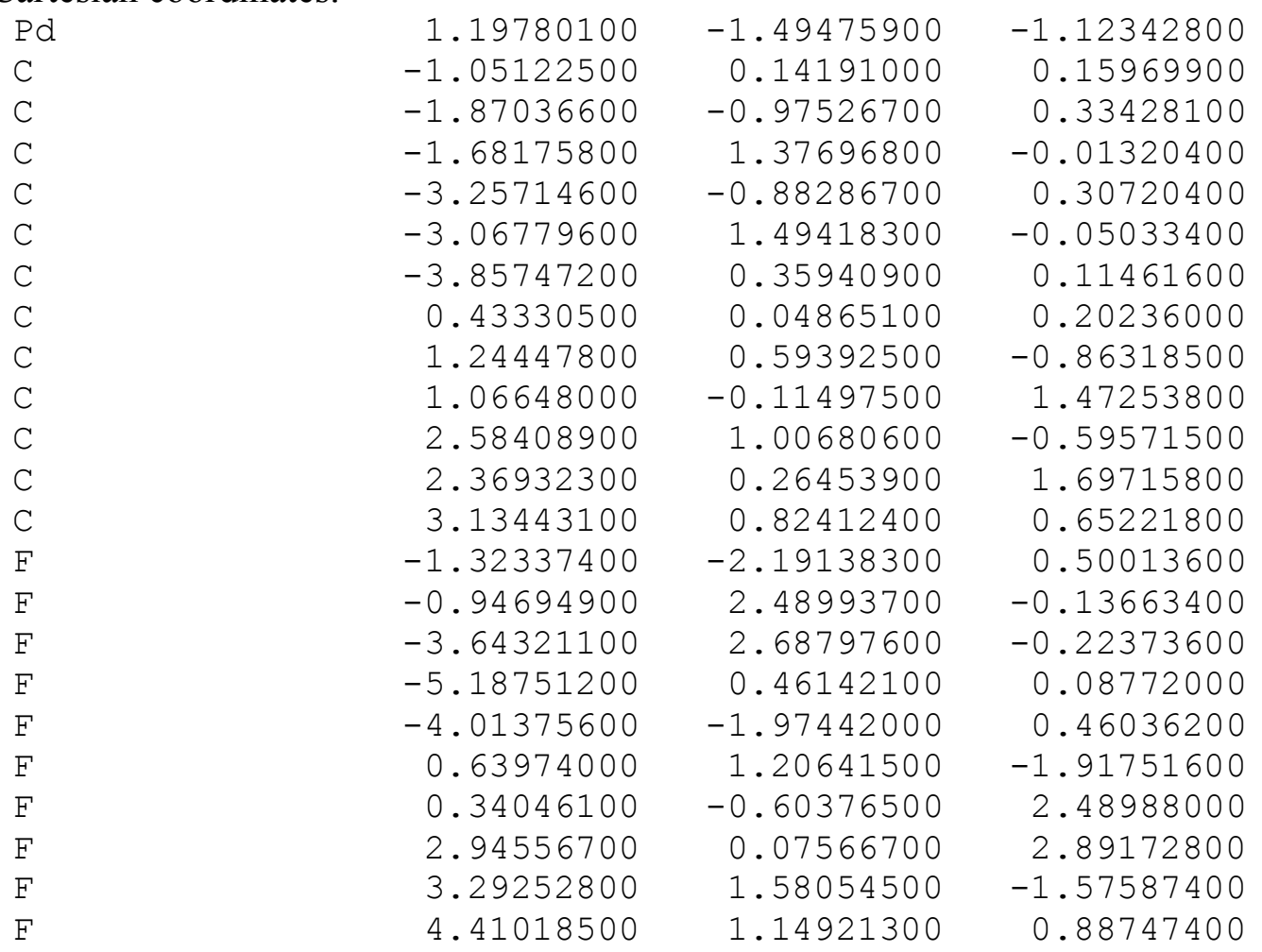

70) $\operatorname{TS}(36-40)$

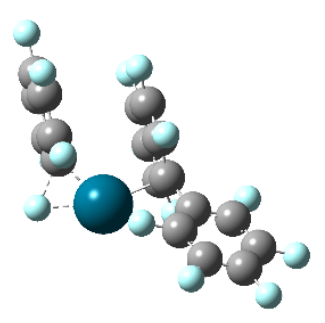

Number of imaginary frequencies $=1\left(i 336.88 \mathrm{~cm}^{-1}\right)$

$\mathrm{E}_{\text {total }}=-2411.14641075$ a.u

$\mathrm{G}_{\text {correction }}=0.087444 \mathrm{a} . \mathrm{u}$

Cartesian coordinates:

$\mathrm{Pd}$

$$
\begin{array}{lll}
-0.06779400 & -0.25764600 & -1.45184000
\end{array}
$$


2.41378800

3. 38566700

2. 85702600

4.74401000

4.21038500

5.15582900

0.97089300

0.19397800

0.45272100

$-0.99701200$

$-0.73240700$

$-1.45895600$

3.01176700

1.97240900

4.60531500

6.45402300

5.64986900

0.64079500

1.25649600

$-1.15935800$

$-1.75046300$

$-2.62803700$

$-2.21494500$

$-2.01204000$

$-2.98742100$

$-4.07550800$

$-4.24577900$

$-3.29982200$

$-4.95630100$

$-5.28848100$

$-3.43621000$

$-1.29764200$

$-1.48165200$

$-2.81963300$
0.14466600

1.11844400

$-1.15294100$

0.82493600

$-1.46978200$

$-0.47593100$

0.49745900

0.02357300

1. 62491900

0.61678400

2.27587200

1.75083000

2. 36642300

$-2.12691600$

$-2.71377000$

$-0.76817800$

1.77385400

$-1.02227200$

2.29657700

3. 34983900

0.11767700

2.28622700

$-1.79234100$

$-0.77802800$

0.21464300

0.26143600

$-0.72995400$

$-1.74813600$

1.26347400

$-0.68407100$

$-2.68138800$

$-2.76658600$

$-1.38040900$

1. 20207500
0.29951400

0.55345900

0.02954000

0.51152800

$-0.01459800$

0.22949800

0.38137700

1. 47880400

$-0.33258600$

1. 82927900

0.08387200

1.13010900

0.86025400

$-0.22980000$

$-0.29111000$

0.19021200

0.75550900

2.17674800

$-1.18154400$

$-0.57635200$

2.81126200

1.47628500

$-0.11034500$

$-1.05186000$

$-1.18394100$

$-0.32009000$

0.64464600

0.75686200

$-0.40874200$

1.48160000

1.70485900

0.03255800

$-2.57411000$

$-2.08382400$

\section{1) Intermediate-40}

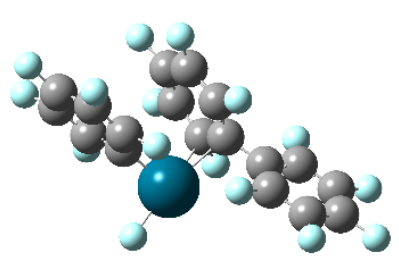

Number of imaginary frequencies $=0$

$\mathrm{E}_{\text {total }}=-2411.18671470 \mathrm{a} . \mathrm{u}$

$\mathrm{G}_{\text {correction }}=0.087562 \mathrm{a} . \mathrm{u}$

\section{Cartesian coordinates:}

$\begin{array}{lr}\mathrm{Pd} & -0.06063000 \\ \mathrm{C} & 2.36767400 \\ \mathrm{C} & 3.40233800 \\ \mathrm{C} & 2.72838400 \\ \mathrm{C} & 4.73651800 \\ \mathrm{C} & 4.05761200\end{array}$

-0.13884200
0.12656700
1.06329300
-1.20809200
0.69975500
-1.59167400

$-1.44228100$

0.35227300

0.47204400

0.12737600

0.33067700

$-0.01361600$ 
5.06365000

0.95218400

0.22014100

0.42344500

$-0.94231900$

$-0.73845300$

$-1.42538300$

3.11893200

1. 78581400

4.37106300

6.33804300

5.70126900

0.68690200

1.18510000

$-1.18042100$

$-1.64782500$

$-2.57385000$

$-2.10951300$

$-1.85871300$

$-2.91434400$

$-4.19482000$

$-4.42655500$

$-3.38167700$

$-5.19836100$

$-5.64933800$

$-3.60615200$

$-1.11740900$

$-0.49801900$

$-2.71264300$

$$
\begin{array}{r}
-0.63315500 \\
0.54171600 \\
0.05977100 \\
1.68949200 \\
0.66521300 \\
2.34203100 \\
1.81053000 \\
2.33929000 \\
-2.14960200 \\
-2.86643200 \\
-0.99000400 \\
1.61321200 \\
-0.99327800 \\
2.35931800 \\
3.42495400 \\
0.16486000 \\
2.34771800 \\
-1.60340200 \\
-0.50029000 \\
0.33690000 \\
0.11816300 \\
-0.97842200 \\
-1.84074300 \\
0.94773000 \\
-1.19689600 \\
-2.88860100 \\
-2.44581700 \\
-1.10005600 \\
1.41147300
\end{array}
$$

0.08853800

0.53374200

1. 65603800

$-0.13101100$

2.07285400

0.33944700

1. 40827700

0.75534500

0.01621200

$-0.24421300$

$-0.04288200$

0.44705300

2.32334300

$-1.01660800$

$-0.28988400$

3.08494900

1.80503000

0.05913500

$-0.74511000$

$-1.07981800$

$-0.57589700$

0.25193900

0.57668500

$-0.87992400$

0.74308400

1.37641800

0.39880800

$-3.05589600$

$-1.86489000$ 


\section{EDA-NOCV Analysis and Results}

Bonding analysis were performed at the B3LYP-D3/TZVP level of theory using the ADF 2017.207 package. $^{\text {S21 }}$ The intrinsic interactions between ligand(s) and metal fragment of various palladium complexes were probed using the combined energy decomposition analysis $(\mathrm{EDA})^{\mathrm{S} 22}$ and natural orbitals for chemical valence (NOCV) ${ }^{\mathrm{S} 23}$ method (EDANOCV) as established by Ziegler et al. ${ }^{\mathrm{S} 24}$ The distortion energy $\left(\Delta E_{\mathrm{dist}}\right)$ is the change in energy between the frozen fragments $\mathrm{A}^{*}$ and $\mathrm{B}^{*}$ of compound $\mathrm{A}-\mathrm{B}$ and the fragments in their equilibrium geometries. The EDA calculations provide information on the intrinsic interaction energy $\left(\Delta E_{\text {int }}\right)$, which is the energy difference between the compound A-B and its frozen fragments $\mathrm{A}^{*}$ and $\mathrm{B}^{*}$. From the EDA calculations, the interaction energy $\Delta E_{\text {int }}$ can be partitioned into three main components $\left(\Delta E_{\text {int }}=\Delta E_{\text {elstat }}+\Delta E_{\text {Pauli }}+\Delta E_{\text {orb }}\right)$. The term $\Delta E_{\text {elstat }}$ is the change in energy due to the quasiclassical electrostatic interaction between the unperturbed charge distributions of the frozen fragments. This $\Delta E_{\text {elstat }}$ part is energetically attractive. The term $\Delta E_{\text {Pauli }}$ stands for the change in energy associated with the Pauli repulsion that comprises the destabilizing interactions between the electrons with same spin on either fragment. Energetically, the $\Delta E_{\text {Pauli }}$ component is repulsive. The term $\Delta E_{\text {orb }}$ accounts for the charge transfer between fragments and polarization effects within each fragment, and represents the attractive energy contribution from each irreducible representation of the point group of the interacting system. The dispersion interaction that arises from the attractive forces between the induced dipoles of interacting fragments is considered to be an important component of chemical bonding; therefore, the energy contribution due to the dispersion interactions $\left(\Delta E_{\text {disp }}\right)$ was computed explicitly, using Grimme's dispersion correction (GD3), ${ }^{18 \mathrm{a}}$ and added as an extra component to $\Delta E_{\text {int. The }}$ $\Delta E_{\text {disp }}$ is, in general, attractive. The coupled EDA-NOCV method aids in partitioning the total orbital interactions into pairwise contributions of the orbital interactions; thus, the nature of 
interaction between fragments can be identified as $\sigma$ donation $\left(\Delta E_{\sigma}\right), \pi$ back-donation $\left(\Delta E_{\pi}\right)$ and intra-fragment polarization $\left(\Delta E_{\text {orb(rest })}\right)$ interactions. Furthermore, the EDA-NOCV analysis demonstrates that most chemical systems usually have a very small number of significant contributions of $\Delta E_{\text {orb. }}$ The deformation density (charge deformation, $\Delta \rho_{k}$ ) that arises from mixing the orbital pairs of the interacting fragments yields information on the amount and shape of charge flow due to the orbital interactions in the compound. The associated energy term $\Delta \mathrm{E}^{k}$ orb gives the strength of the orbital interactions. Thus, with the EDA-NOCV analysis, both qualitative $\left(\Delta \rho_{k}\right)$ and quantitative $\left(\Delta E_{\text {orb }}^{k}\right)$ details of the strength of individual pairs of orbital interactions can be obtained. More details on the EDA and EDA-NOCV methods, terms and symbols used, and their applications for bonding analysis can be found in the literature. ${ }^{\mathrm{S} 25}$

\section{(a) Numerical results from EDA-NOCV analysis:}

Table S3. EDA-NOCV results of the chemical bonding in 3a, TS(3a-4), 7, TS(7-8), 12, TS(12-13), 16, and TS(16-17) at the B3LYP-D3/TZVP level of theory. Energies are in $\mathrm{kcal} / \mathrm{mol}$. The first fragment is $\mathrm{Pd}\left(\mathrm{Ar}_{\mathrm{F}}\right)_{2}$ for all, and the other fragment(s) is/are MeCN for 3a and TS(3a-4), THF for 7 and TS(7-8), $\mathrm{SMe}_{2}$ for 12 and TS(12-13), and $\mathrm{PMe}_{3}$ for 16 and

\begin{tabular}{|c|c|c|c|c|c|c|c|c|}
\hline Structure & $3 a$ & $\operatorname{TS}(3 a-4)$ & 7 & TS(7-8) & 12 & $\operatorname{TS}(12-13)$ & 16 & $\operatorname{TS}(16-17)$ \\
\hline$\Delta E_{\text {int }}$ & -55.3 & -35.4 & -58.1 & -36.8 & -70.8 & -52.1 & -102.0 & -77.0 \\
\hline$\Delta E_{\text {Pauli }}$ & 130.6 & 106.5 & 102.5 & 80.0 & 162.7 & 163.9 & 280.0 & 281.1 \\
\hline$\Delta E_{\text {disp }}$ & -8.2 & -7.0 & -20.8 & -20.7 & -21.7 & -20.6 & -27.2 & -24.1 \\
\hline$\Delta E_{\text {elstat }^{\mathrm{a}}}$ & $\begin{array}{l}-117.1 \\
(65.9 \%)\end{array}$ & $\begin{array}{l}-89.8 \\
(66.6 \%)\end{array}$ & $\begin{array}{l}-95.6 \\
(68.4 \%)\end{array}$ & $\begin{array}{l}-68.3 \\
(71.1 \%)\end{array}$ & $\begin{array}{l}-141.2 \\
(66.7 \%)\end{array}$ & $\begin{array}{l}-141.0 \\
(72.1 \%)\end{array}$ & $\begin{array}{l}-250.1 \\
(70.5 \%)\end{array}$ & $\begin{array}{l}-252.6 \\
(75.6 \%)\end{array}$ \\
\hline$\Delta E_{\text {orb }^{\mathrm{a}}}$ & $\begin{array}{l}-60.6 \\
(34.1 \%)\end{array}$ & $\begin{array}{l}-45.1 \\
(33.4 \%)\end{array}$ & $\begin{array}{l}-44.2 \\
(31.6 \%)\end{array}$ & $\begin{array}{l}-27.8 \\
(28.9 \%)\end{array}$ & $\begin{array}{l}-70.6 \\
(33.3 \%)\end{array}$ & $\begin{array}{l}-54.5 \\
(27.9 \%)\end{array}$ & $\begin{array}{l}-104.7 \\
(29.5 \%)\end{array}$ & $\begin{array}{l}-81.4 \\
(24.4 \%)\end{array}$ \\
\hline$\Delta E^{\mathbf{~}_{\text {orb }}}{ }^{\mathrm{b}}$ & $\begin{array}{l}-24.5 \\
(40.4 \%)\end{array}$ & $\begin{array}{l}-16.2 \\
(35.9 \%)\end{array}$ & $\begin{array}{l}-19.8 \\
(44.8 \%)\end{array}$ & $\begin{array}{l}-11.5 \\
(41.4 \%)\end{array}$ & $\begin{array}{l}-33.0 \\
(46.7 \%)\end{array}$ & $\begin{array}{l}-17.2 \\
(31.6 \%)\end{array}$ & $\begin{array}{l}-51.9 \\
(49.6 \%)\end{array}$ & $\begin{array}{l}-24.9 \\
(30.6 \%)\end{array}$ \\
\hline$\Delta E^{2}{ }_{\text {orb }}{ }^{b}$ & $\begin{array}{l}-9.5 \\
(15.7 \%)\end{array}$ & $\begin{array}{l}-10.4 \\
(23.1 \%)\end{array}$ & $\begin{array}{l}-9.3 \\
(21.0 \%)\end{array}$ & $\begin{array}{l}-6.6 \\
(23.7 \%)\end{array}$ & $\begin{array}{l}-14.4 \\
(20.4 \%)\end{array}$ & $\begin{array}{l}-14.9 \\
(27.3 \%)\end{array}$ & $\begin{array}{l}-19.4 \\
(18.5 \%)\end{array}$ & $\begin{array}{l}-23.9 \\
(29.4 \%)\end{array}$ \\
\hline$\Delta E^{3}$ orb $^{\text {b }}$ & $\begin{array}{l}-7.7 \\
(12.7 \%)\end{array}$ & $\begin{array}{l}-3.8 \\
(8.4 \%)\end{array}$ & $-c$ & $-\mathrm{c}$ & $\begin{array}{l}-5.0 \\
(7.1 \%)\end{array}$ & $\begin{array}{l}-5.6 \\
(10.3 \%)\end{array}$ & $\begin{array}{l}-7.9 \\
(7.5 \%)\end{array}$ & $\begin{array}{l}-8.8 \\
(10.8 \%)\end{array}$ \\
\hline$\Delta E^{4}$ orb $^{\mathrm{b}}$ & $\begin{array}{l}-4.5 \\
(7.4 \%)\end{array}$ & $\begin{array}{l}-4.8 \\
(10.6 \%)\end{array}$ & $-\mathrm{c}$ & $-\mathrm{c}$ & $\begin{array}{l}-3.9 \\
(5.5 \%)\end{array}$ & $\begin{array}{l}-4.0 \\
(7.3 \%)\end{array}$ & $\begin{array}{l}-5.4 \\
(5.2 \%)\end{array}$ & $\begin{array}{l}-11.9 \\
(14.6 \%)\end{array}$ \\
\hline$\Delta E^{5_{o r b}}{ }^{b}$ & $\begin{array}{l}-4.1 \\
(6.8 \%)\end{array}$ & $-c$ & $-c$ & $-c$ & $-c$ & $-c$ & $\begin{array}{l}-5.1 \\
(4.9 \%)\end{array}$ & $-c$ \\
\hline$\Delta E_{\sigma}{ }^{\mathbf{b}}$ & $\begin{array}{l}-41.7 \\
(68.8 \%)\end{array}$ & $\begin{array}{l}-21.1 \\
(46.8 \%)\end{array}$ & $\begin{array}{l}-29.1 \\
(65.8 \%)\end{array}$ & $\begin{array}{l}-18.1 \\
(65.1 \%)\end{array}$ & $\begin{array}{l}-47.4 \\
(67.1 \%)\end{array}$ & $\begin{array}{l}-37.7 \\
(69.2 \%)\end{array}$ & $\begin{array}{l}-79.2 \\
(75.6 \%)\end{array}$ & $\begin{array}{l}-57.6 \\
(70.8 \%)\end{array}$ \\
\hline$\Delta E_{\pi}{ }^{\mathbf{b}}$ & $\begin{array}{l}-8.6 \\
(14.2 \%)\end{array}$ & $\begin{array}{l}-14.2 \\
(31.5 \%)\end{array}$ & $-c$ & $-c$ & $\begin{array}{l}-3.9 \\
(5.5 \%)\end{array}$ & $\begin{array}{l}-4.0 \\
(7.3 \%)\end{array}$ & $\begin{array}{l}-10.5 \\
(10.0 \%)\end{array}$ & $\begin{array}{l}-11.9 \\
(14.6 \%)\end{array}$ \\
\hline$\Delta E_{\text {orb(rest) }}{ }^{b}$ & $\begin{array}{l}-10.3 \\
(17.0 \%)\end{array}$ & $\begin{array}{l}-9.9 \\
(22.0 \%)\end{array}$ & $\begin{array}{l}-15.3 \\
(34.6 \%)\end{array}$ & $\begin{array}{l}-9.7 \\
(34.9 \%)\end{array}$ & $\begin{array}{l}-19.3 \\
(27.3 \%)\end{array}$ & $\begin{array}{l}-12.8 \\
(23.5 \%)\end{array}$ & $\begin{array}{l}-15.0 \\
(14.3 \%)\end{array}$ & $\begin{array}{l}-11.9 \\
(14.6 \%)\end{array}$ \\
\hline
\end{tabular}

${ }^{\mathrm{a}}$ The value in parenthesis denote the percentage contribution to the total attractive interaction $\left(\Delta E_{\text {elstat }}+\Delta E_{\text {orb }}\right) .{ }^{\mathrm{b}} \mathrm{The}$ value in parenthesis denote the percentage contribution to the total orbital interaction $\left(\Delta E_{\mathrm{orb}}\right)$. ${ }^{\mathrm{c}}$ No or insignificant energy contribution was found. 
Table S4. EDA-NOCV results of the chemical bonding in 20, TS(20-21), 25, TS(25-26), 30, TS(30-31), 35, and TS(35-36) at the B3LYP-D3/TZVP level of theory. Energies are in kcal/mol. The first fragment is $\mathrm{Pd}_{(}\left(\mathrm{Ar}_{\mathrm{F}}\right)_{2}$ for all, and the other fragment is benzene for $\mathbf{2 0}$ and TS(20-21), toluene for 25 and TS(25-26), $m$-xylene for 30 and TS(30-31), and perfluorobenzene for 35 and TS(35-36).

\begin{tabular}{|c|c|c|c|c|c|c|c|c|}
\hline Structure & 20 & TS(20-21) & 25 & $\operatorname{TS}(25-26)$ & 30 & TS(30-31) & 35 & TS(35-36) \\
\hline$\Delta E_{\text {int }}$ & -27.4 & -26.2 & -30.3 & -27.5 & -32.6 & -28.4 & -12.2 & -16.9 \\
\hline$\Delta E_{\text {Pauli }}$ & 64.5 & 66.8 & 67.8 & 68.0 & 69.1 & 68.3 & 53.6 & 68.9 \\
\hline$\Delta E_{\text {disp }}$ & -11.3 & -8.6 & -12.9 & -9.2 & -13.6 & -10.7 & -11.6 & -14.2 \\
\hline$\Delta E_{\text {elstat }^{a}}$ & $\begin{array}{l}-45.5 \\
(56.5 \%)\end{array}$ & $\begin{array}{l}-53.1 \\
(62.9 \%)\end{array}$ & $\begin{array}{l}-48.5 \\
(57.0 \%)\end{array}$ & $\begin{array}{l}-54.6 \\
(63.2 \%)\end{array}$ & $\begin{array}{l}-50.5 \\
(57.3 \%)\end{array}$ & $\begin{array}{l}-55.2 \\
(64.3 \%)\end{array}$ & $\begin{array}{l}-25.4 \\
(46.8 \%)\end{array}$ & $\begin{array}{l}-41.5 \\
(58.0 \%)\end{array}$ \\
\hline$\Delta E_{o r b}{ }^{\mathrm{a}}$ & $\begin{array}{l}-35.0 \\
(43.5 \%)\end{array}$ & $\begin{array}{l}-31.3 \\
(37.1 \%)\end{array}$ & $\begin{array}{l}-36.6 \\
(43.0 \%)\end{array}$ & $\begin{array}{l}-31.8 \\
(36.8 \%)\end{array}$ & $\begin{array}{l}-37.6 \\
(42.7 \%)\end{array}$ & $\begin{array}{l}-30.7 \\
(35.7 \%)\end{array}$ & $\begin{array}{l}-28.9 \\
(53.2 \%)\end{array}$ & $\begin{array}{l}-30.1 \\
(42.0 \%)\end{array}$ \\
\hline$\Delta E^{1}{ }_{\text {orb }}{ }^{\mathrm{b}}$ & $\begin{array}{l}-12.0 \\
(34.3 \%)\end{array}$ & $-9.8(31.3 \%)$ & $\begin{array}{l}-12.8 \\
(35.0 \%)\end{array}$ & $-9.6(30.2 \%)$ & $\begin{array}{l}-13.4 \\
(35.6 \%)\end{array}$ & $-8.6(28.0 \%)$ & $\begin{array}{l}-9.1 \\
(31.5 \%)\end{array}$ & $\begin{array}{l}-11.6 \\
(37.9 \%)\end{array}$ \\
\hline$\Delta E^{2}$ orb $^{\mathrm{b}}$ & $\begin{array}{l}-7.2 \\
(20.6 \%)\end{array}$ & $-9.7(31.0 \%)$ & $\begin{array}{l}-7.2 \\
(19.7 \%)\end{array}$ & $-9.8(30.8 \%)$ & $\begin{array}{l}-7.4 \\
(19.7 \%)\end{array}$ & $-9.7(31.6 \%)$ & $\begin{array}{l}-5.9 \\
(20.4 \%)\end{array}$ & $-7.7(25.6 \%)$ \\
\hline$\Delta E^{3}$ orb $^{\mathrm{b}}$ & $\begin{array}{l}-3.0 \\
(8.6 \%)\end{array}$ & $-4.5(14.4 \%)$ & $\begin{array}{l}-3.2 \\
(8.7 \%)\end{array}$ & $-4.7(14.8 \%)$ & $\begin{array}{l}-3.3 \\
(8.8 \%)\end{array}$ & $-4.5(14.7 \%)$ & $\begin{array}{l}-3.4 \\
(11.8 \%)\end{array}$ & $-4.0(13.3 \%)$ \\
\hline$\Delta E^{4}{ }^{4} b^{b}$ & $\begin{array}{l}-3.6 \\
(10.3 \%)\end{array}$ & $\begin{array}{l}-2.6 \\
(8.3 \%)\end{array}$ & $\begin{array}{l}-3.4 \\
(9.3 \%)\end{array}$ & $\begin{array}{l}-2.9 \\
(9.1 \%)\end{array}$ & $-c$ & $\begin{array}{l}-2.9 \\
(9.4 \%)\end{array}$ & $\begin{array}{l}-2.1 \\
(7.3 \%)\end{array}$ & $\begin{array}{l}-2.0 \\
(6.6 \%)\end{array}$ \\
\hline$\Delta E_{\sigma} \mathbf{b}$ & $\begin{array}{l}-22.8 \\
(65.1 \%)\end{array}$ & $\begin{array}{l}-14.2 \\
(45.4 \%)\end{array}$ & $\begin{array}{l}-23.4 \\
(63.9 \%)\end{array}$ & $\begin{array}{l}-14.4 \\
(45.3 \%)\end{array}$ & $\begin{array}{l}-20.8 \\
(55.3 \%)\end{array}$ & $\begin{array}{l}-14.1 \\
(45.9 \%)\end{array}$ & $\begin{array}{l}-18.4 \\
(65.4 \%)\end{array}$ & $-9.7(32.2 \%)$ \\
\hline$\Delta E_{\pi}{ }^{b}$ & $\begin{array}{l}-3.0 \\
(8.6 \%)\end{array}$ & $\begin{array}{l}-12.4 \\
(39.6 \%)\end{array}$ & $\begin{array}{l}-3.2 \\
(8.7 \%)\end{array}$ & $\begin{array}{l}-12.5 \\
(39.3 \%)\end{array}$ & $\begin{array}{l}-3.3 \\
(8.8 \%)\end{array}$ & $\begin{array}{l}-11.5 \\
(37.5 \%)\end{array}$ & $\begin{array}{l}-2.1 \\
(7.3 \%)\end{array}$ & $\begin{array}{l}-15.5 \\
(51.5 \%)\end{array}$ \\
\hline$\Delta E_{\text {orb(rest) }}{ }^{\mathrm{b}}$ & $\begin{array}{l}-9.2 \\
(26.3 \%)\end{array}$ & -4.7 (15.0\%) & $\begin{array}{l}-10.0 \\
(27.3 \%)\end{array}$ & $-4.8(15.1 \%)$ & $\begin{array}{l}-13.5 \\
(35.9 \%)\end{array}$ & $-5.0(16.3 \%)$ & $\begin{array}{l}-8.4 \\
(29.1 \%)\end{array}$ & $-4.8(15.9 \%)$ \\
\hline
\end{tabular}

\section{(b) Plots of deformation density, associated energy and the orbitals involved in the interaction}

The important deformation densities $\left(\Delta \rho_{k}\right)$, which show the change in the electronic structure that arise from the pairwise orbital interactions among the fragments in their closed-shell state, and that are associated with significant orbital interactions in all reactants and selected transition states, are provided below in the first row. The associated energy ( $\left.\Delta E_{\text {orb }}^{k}\right)$ gained from each charge deformation upon orbital interaction between the fragments and the respective eigen value $(v)$, representing the size of the charge flow, are also given in the same row. The color code for the direction of charge flow (charge transfer) in the deformation density plots is red to blue. Information concerning the type of orbital interaction and the orbitals of fragments engaged in that interaction, which are derived from deformation densities and eigenvalues, are given in the second row. 
For cis- $\left[\mathrm{Pd}(\mathrm{MeCN})_{2}\left(\mathrm{C}_{6} \mathrm{~F}_{5}\right)_{2}\right]$ (3a):

\begin{tabular}{|c|c|c|c|c|c|}
\hline $\begin{array}{l}\text { Deformatio } \\
\text { n density }\end{array}$ & $\begin{array}{l}\Delta \rho_{1} ; \Delta E_{\text {orb }}^{1}=-24.5 \\
\mathrm{kcal} / \mathrm{mol} ; \mathrm{v}= \pm 0.46\end{array}$ & $\begin{array}{l}\Delta \rho_{2} ; \Delta E_{\mathrm{orb}}^{2}=-9.5 \\
\mathrm{kcal} / \mathrm{mol} ; v= \pm 0.26\end{array}$ & $\begin{array}{l}\text { ( } \rho_{3} ; \Delta E_{\mathrm{orb}}^{3}=-7.7 \\
\mathrm{kcal} / \mathrm{mol} ; \mathrm{v}= \pm \\
0.22\end{array}$ & $\begin{array}{l}\text { 8. } \\
\Delta \rho_{4} ; \Delta E_{\text {orb }}^{4}=-4.5 \\
\mathrm{kcal} / \mathrm{mol} ; v= \pm \\
0.21\end{array}$ & $\begin{array}{l}\Delta \rho_{5} ; \Delta E_{\mathrm{orb}}^{5}=-4.1 \\
\mathrm{kcal} / \mathrm{mol} ; \mathrm{v}= \pm \\
0.20\end{array}$ \\
\hline $\begin{array}{l}\text { Type of } \\
\text { interaction } \\
\text { and the } \\
\text { orbitals of } \\
\text { fragments } \\
\text { involved in } \\
\text { the } \\
\text { interaction }\end{array}$ & $\begin{array}{l}\text { o-donation } \\
\text { (HOMO-2 of } \\
\mathrm{MeCN}-1 \\
\text { and } \mathrm{MeCN}-2 \\
\text { fragments } \\
\text { to LUMO of } \\
\mathrm{Pd}\left(\mathrm{Ar}_{\mathrm{F}}\right)_{2} \text { fragment) }\end{array}$ & $\begin{array}{l}\text { o-donation } \\
\text { (HOMO-2 of } \\
\mathrm{MeCN}-1 \\
\text { and } \mathrm{MeCN}-2 \\
\text { fragments } \\
\text { to } \mathrm{LUMO}+1 \text { of } \\
\mathrm{Pd}\left(\mathrm{Ar}_{\mathrm{F}}\right)_{2} \text { fragment) }\end{array}$ & $\begin{array}{l}\text { б-donation } \\
\text { (HOMO-2 of } \\
\text { MeCN-1 } \\
\text { and MeCN-2 } \\
\text { fragments } \\
\text { to } \mathrm{LUMO}_{\mathrm{H}} 1 \text { of } \\
\mathrm{Pd}\left(\mathrm{Ar}_{\mathrm{F}}\right)_{2} \\
\text { fragment) }\end{array}$ & $\begin{array}{l}\pi \text { back-donation } \\
(\mathrm{HOMO}-6 \text { of } \\
\mathrm{Pd}\left(\mathrm{Ar}_{\mathrm{F}}\right)_{2} \text { fragment } \\
\text { to } \mathrm{LUMO}+2 \text { of } \\
\mathrm{MeCN}-1 \text { and } \\
\mathrm{MeCN}-2 \\
\text { fragments) }\end{array}$ & $\begin{array}{l}\pi \text { back-donation } \\
(\mathrm{HOMO}-6 \text { of } \\
\mathrm{Pd}\left(\mathrm{Ar}_{\mathrm{F}}\right)_{2} \text { fragment } \\
\text { to } \mathrm{LUMO}+2 \text { of } \\
\mathrm{MeCN}-1 \text { and } \\
\mathrm{MeCN}-2 \\
\text { fragments })\end{array}$ \\
\hline
\end{tabular}

For TS(3a-4):

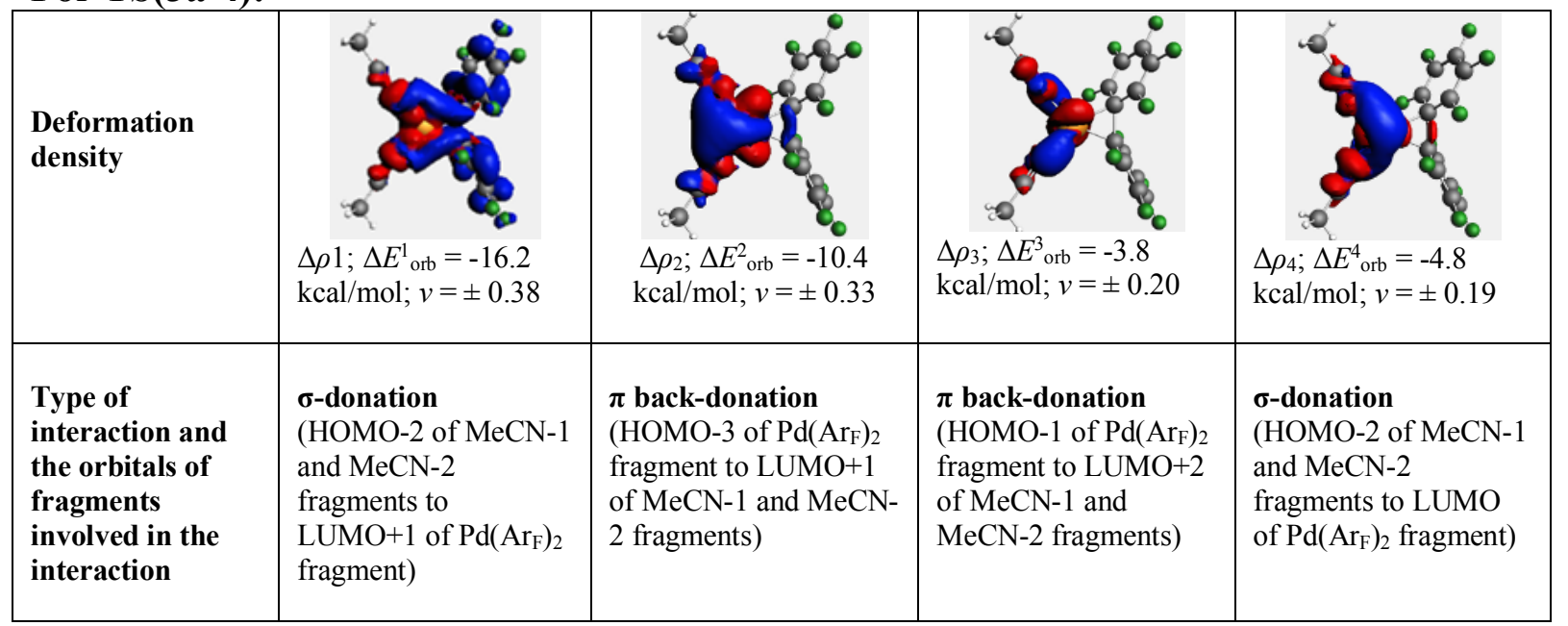


For cis-[Pd(THF $\left.)_{2}\left(\mathrm{C}_{6} \mathrm{~F}_{5}\right)_{2}\right](\mathbf{7})$ :

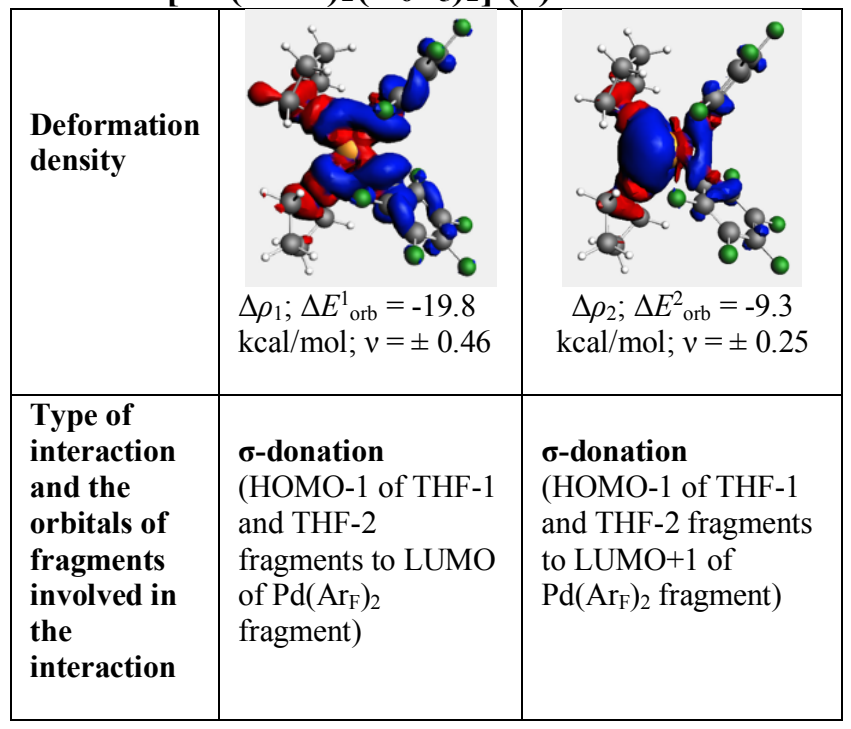

\section{For TS(7-8):}

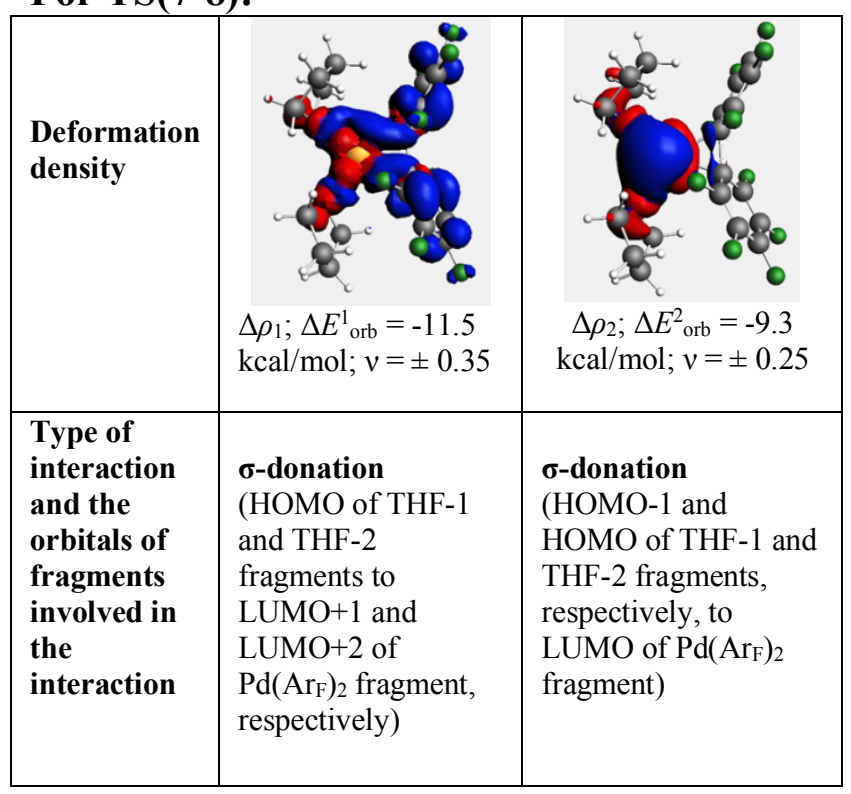


For cis- $\left[\mathrm{Pd}\left(\mathrm{SMe}_{2}\right)_{2}\left(\mathrm{C}_{6} \mathrm{~F}_{5}\right)_{2}\right]$ (12):

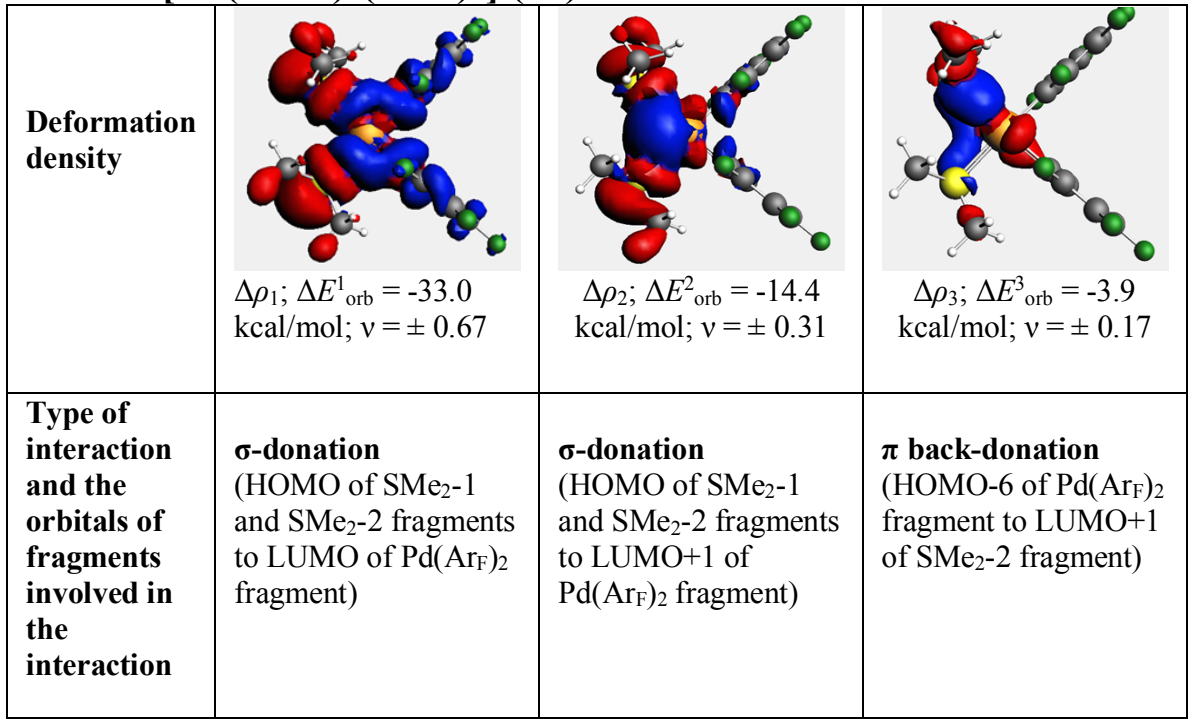

\section{For TS(12-13):}

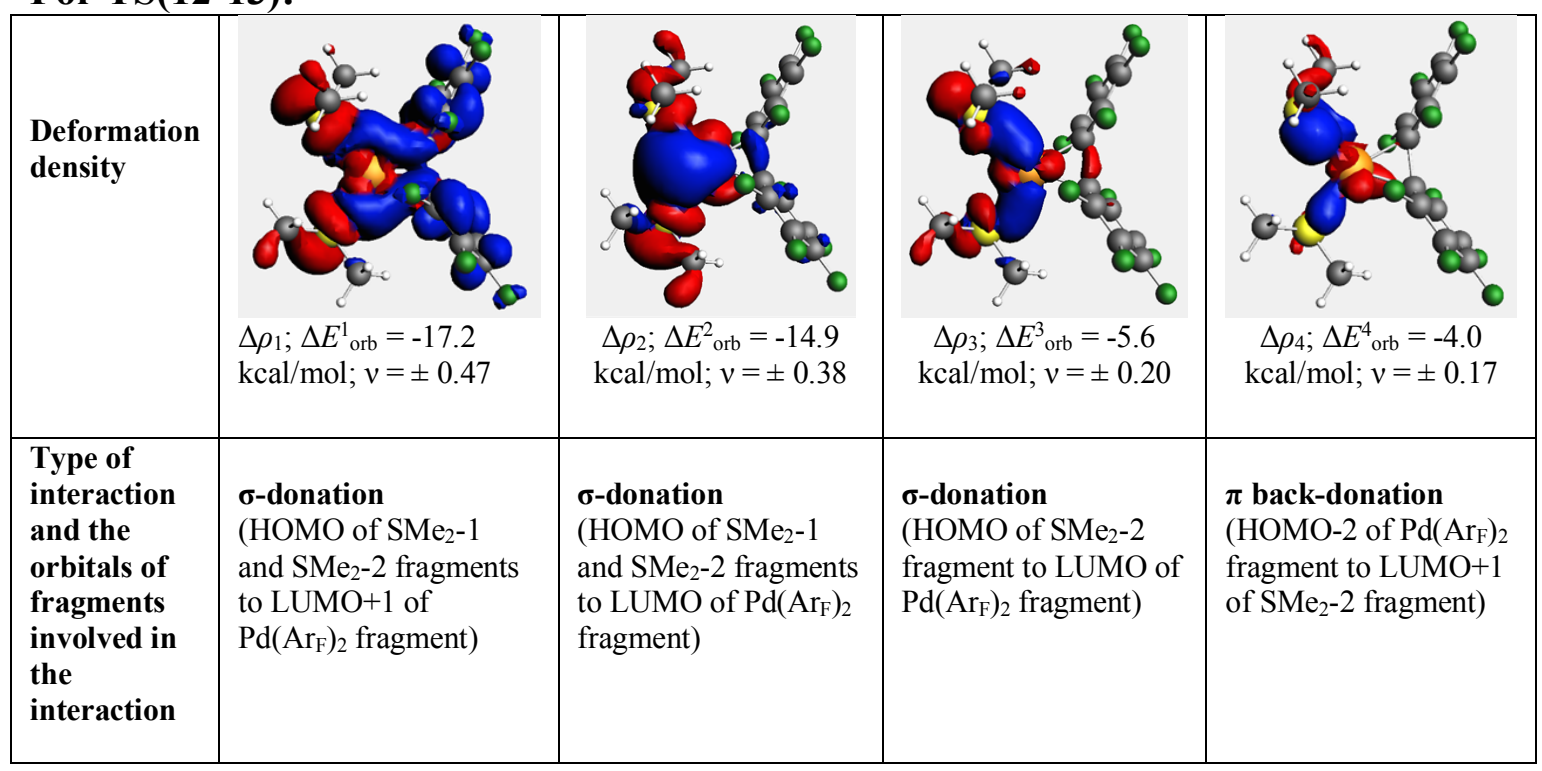


For cis- $\left[\mathrm{Pd}\left(\mathrm{PMe}_{3}\right)_{2}\left(\mathrm{C}_{6} \mathrm{~F}_{5}\right)_{2}\right](\mathbf{1 6})$ :

\begin{tabular}{|c|c|c|c|c|c|}
\hline $\begin{array}{l}\text { Deformation } \\
\text { density }\end{array}$ & $\begin{array}{l}\Delta \rho_{1} ; \Delta E_{\text {orb }}^{1}=- \\
51.9 \mathrm{kcal} / \mathrm{mol} ; v \\
= \pm 0.84\end{array}$ & $\begin{array}{l}\Delta \rho_{2} ; \Delta E_{\mathrm{orb}}^{2}=- \\
19.4 \mathrm{kcal} / \mathrm{mol} ; v= \\
\pm 0.38\end{array}$ & $\begin{aligned} \Delta \rho_{3} ; \Delta E^{3} \text { orb } & =-7.9 \\
\mathrm{kcal} / \mathrm{mol} ; \mathrm{v} & = \pm .23\end{aligned}$ & $\begin{array}{l}\Delta \rho_{4} ; \Delta E_{\text {orb }}^{4}=-5.4 \\
\mathrm{kcal} / \mathrm{mol} ; v= \pm \\
0.20\end{array}$ & $\begin{array}{l}080 \\
\Delta \rho_{5} ; \Delta E_{\text {orb }}^{5}=-5.1 \\
\mathrm{kcal} / \mathrm{mol} ; v= \pm \\
0.20\end{array}$ \\
\hline $\begin{array}{l}\text { Type of } \\
\text { interaction } \\
\text { and the } \\
\text { orbitals of } \\
\text { fragments } \\
\text { involved in } \\
\text { the } \\
\text { interaction }\end{array}$ & $\begin{array}{l}\text { o-donation } \\
\text { (HOMO of } \\
\mathrm{PMe}_{3}-1 \text { and } \\
\mathrm{PMe}_{3}-2 \\
\text { fragments to } \\
\mathrm{LUMO} \text { of } \\
\mathrm{Pd}\left(\mathrm{Ar}_{\mathrm{F}}\right)_{2} \\
\text { fragment) }\end{array}$ & $\begin{array}{l}\text { б-donation } \\
\text { ( } \mathrm{HOMO} \text { of } \mathrm{PMe}_{3}- \\
1 \text { and } \mathrm{PMe}_{3}-2 \\
\text { fragments to } \\
\mathrm{LUMO}+1 \text { of } \\
\mathrm{Pd}\left(\mathrm{Ar}_{\mathrm{F}}\right)_{2} \\
\text { fragment) }\end{array}$ & 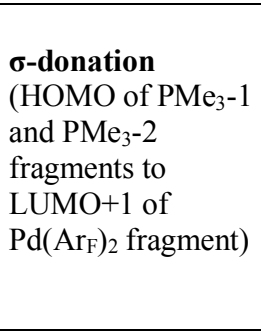 & $\begin{array}{l}\boldsymbol{\pi} \text { back-donation } \\
(\mathrm{HOMO}-5 \text { of } \\
\mathrm{Pd}\left(\mathrm{Ar}_{\mathrm{F}}\right)_{2} \text { fragment } \\
\text { to } \mathrm{LUMO}+8 \text { of } \\
\mathrm{PMe}_{3}-1 \text { and } \\
\mathrm{PMe}_{3}-2 \\
\text { fragments) }\end{array}$ & $\begin{array}{l}\pi \text { back-donation } \\
(\mathrm{HOMO}-6 \text { of } \\
\mathrm{Pd}\left(\mathrm{Ar}_{\mathrm{F}}\right)_{2} \text { fragment } \\
\text { to } \mathrm{LUMO}+8 \text { of } \\
\mathrm{PMe}_{3}-1 \text { and } \mathrm{PMe}_{3}- \\
2 \text { fragments) }\end{array}$ \\
\hline
\end{tabular}

For TS(16-17):

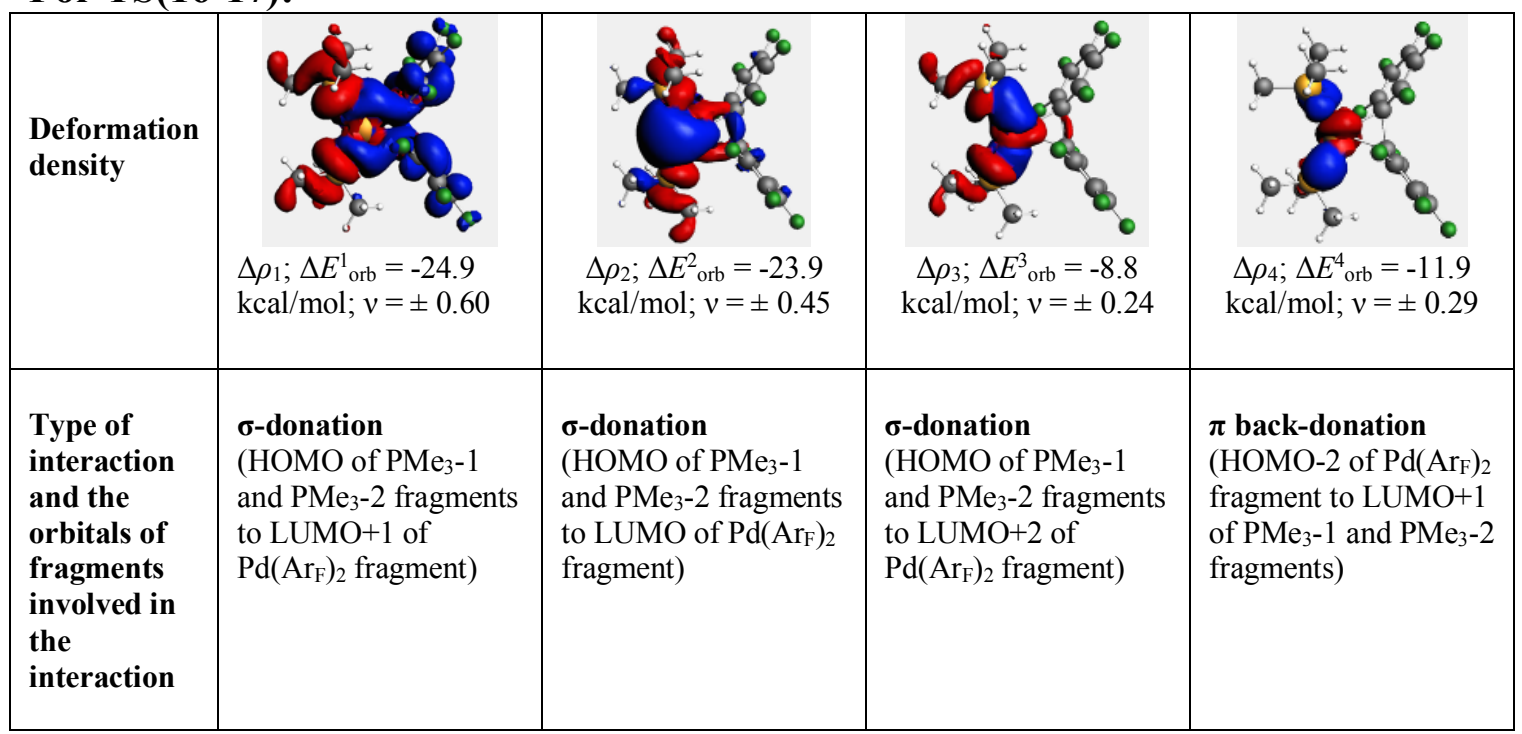


For cis- $\left[\mathrm{Pd}\left(\mathrm{C}_{6} \mathrm{H}_{6}\right)\left(\mathrm{C}_{6} \mathrm{~F}_{5}\right)_{2}\right](20)$ :

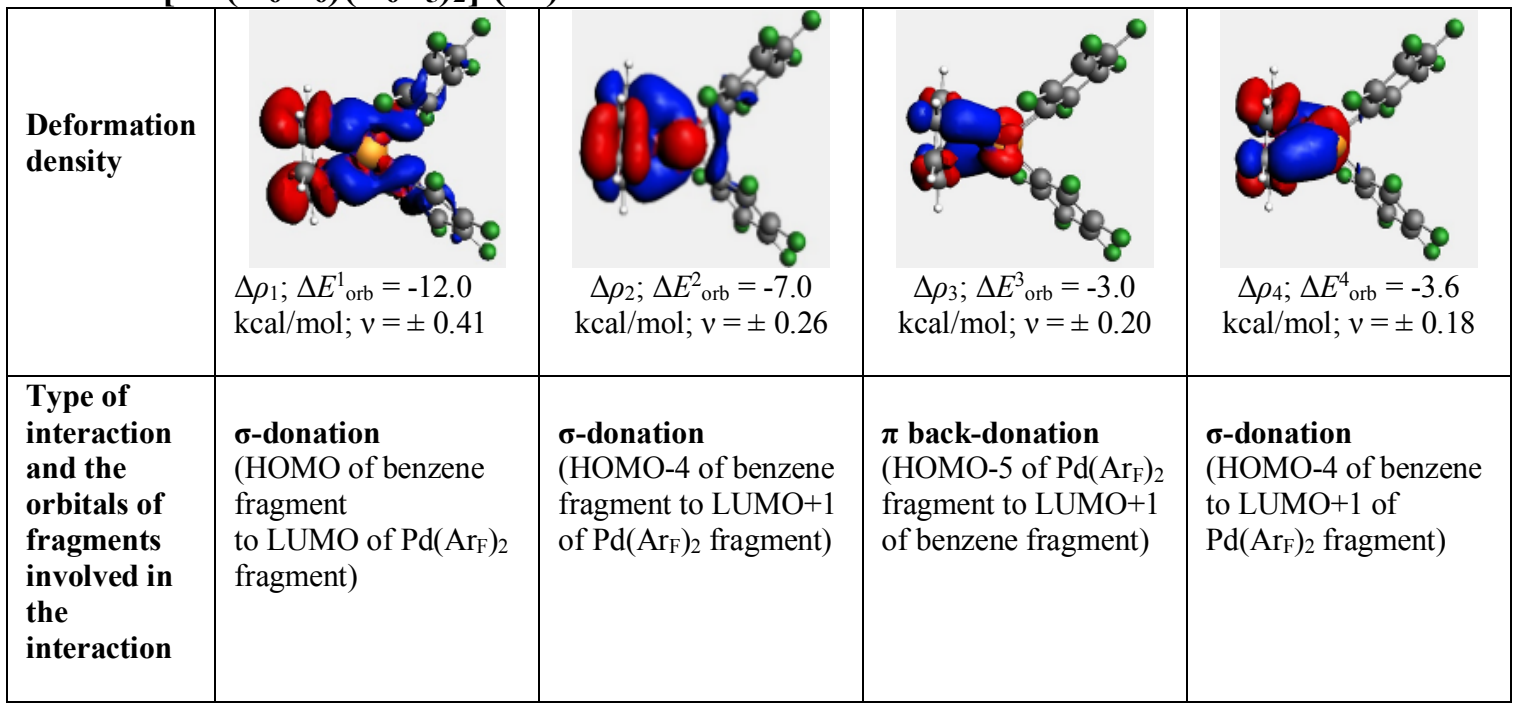

\section{For TS(20-21):}

\begin{tabular}{|c|c|c|c|c|}
\hline $\begin{array}{l}\text { Deformation } \\
\text { density }\end{array}$ & $\begin{array}{l}\text { kcal } / \mathrm{mol} ; v= \pm 0.33 \\
\Delta \rho_{1} ; \Delta E_{\text {orb }}=-9.8 \\
\text { afse }\end{array}$ & $\begin{array}{l}\Delta \rho_{2} ; \Delta E^{2} \text { orb }=-9.7 \\
\mathrm{kcal} / \mathrm{mol} ; \mathrm{v}= \pm 0.28\end{array}$ & $\begin{array}{c}\text { ofo } \\
\Delta \rho_{3} ; \Delta E_{\text {orb }}^{3}=-4.5 \\
\mathrm{kcal} / \mathrm{mol} ; \mathrm{v}= \pm 0.22\end{array}$ & $\begin{array}{c}\Delta \rho_{4} ; \Delta E^{4}{ }_{\mathrm{orb}}=-2.6 \\
\mathrm{kcal} / \mathrm{mol} ; \mathrm{v}= \pm 0.19\end{array}$ \\
\hline $\begin{array}{l}\text { Type of } \\
\text { interaction } \\
\text { and the } \\
\text { orbitals of } \\
\text { fragments } \\
\text { involved in } \\
\text { the } \\
\text { interaction }\end{array}$ & $\begin{array}{l}\boldsymbol{\pi} \text { back-donation } \\
(\mathrm{HOMO}-3 \text { and } \\
\mathrm{HOMO}-6 \text { of } \mathrm{Pd}\left(\mathrm{Ar}_{\mathrm{F}}\right)_{2} \\
\text { fragment to LUMO of } \\
\text { benzene fragment) }\end{array}$ & $\begin{array}{l}\text { б-donation } \\
\text { (HOMO of benzene } \\
\text { fragment to LUMO of } \\
\operatorname{Pd}\left(\mathrm{Ar}_{\mathrm{F}}\right)_{2} \text { fragment) }\end{array}$ & $\begin{array}{l}\text { o-donation } \\
\text { (HOMO- } 1 \text { of benzene } \\
\text { fragment to LUMO+1 } \\
\text { of } \operatorname{Pd}\left(\mathrm{Ar}_{\mathrm{F}}\right)_{2} \text { fragment) }\end{array}$ & $\begin{array}{l}\boldsymbol{\pi} \text { back-donation } \\
\left(\mathrm{HOMO} \text { of } \mathrm{Pd}\left(\mathrm{Ar}_{\mathrm{F}}\right)_{2}\right. \\
\text { fragment to } \mathrm{LUMO}+1 \\
\text { of benzene })\end{array}$ \\
\hline
\end{tabular}


For cis- $\left[\mathrm{Pd}\left(\mathrm{C}_{7} \mathrm{H}_{8}\right)\left(\mathrm{C}_{6} \mathrm{~F}_{5}\right)_{2}\right](25)$ :

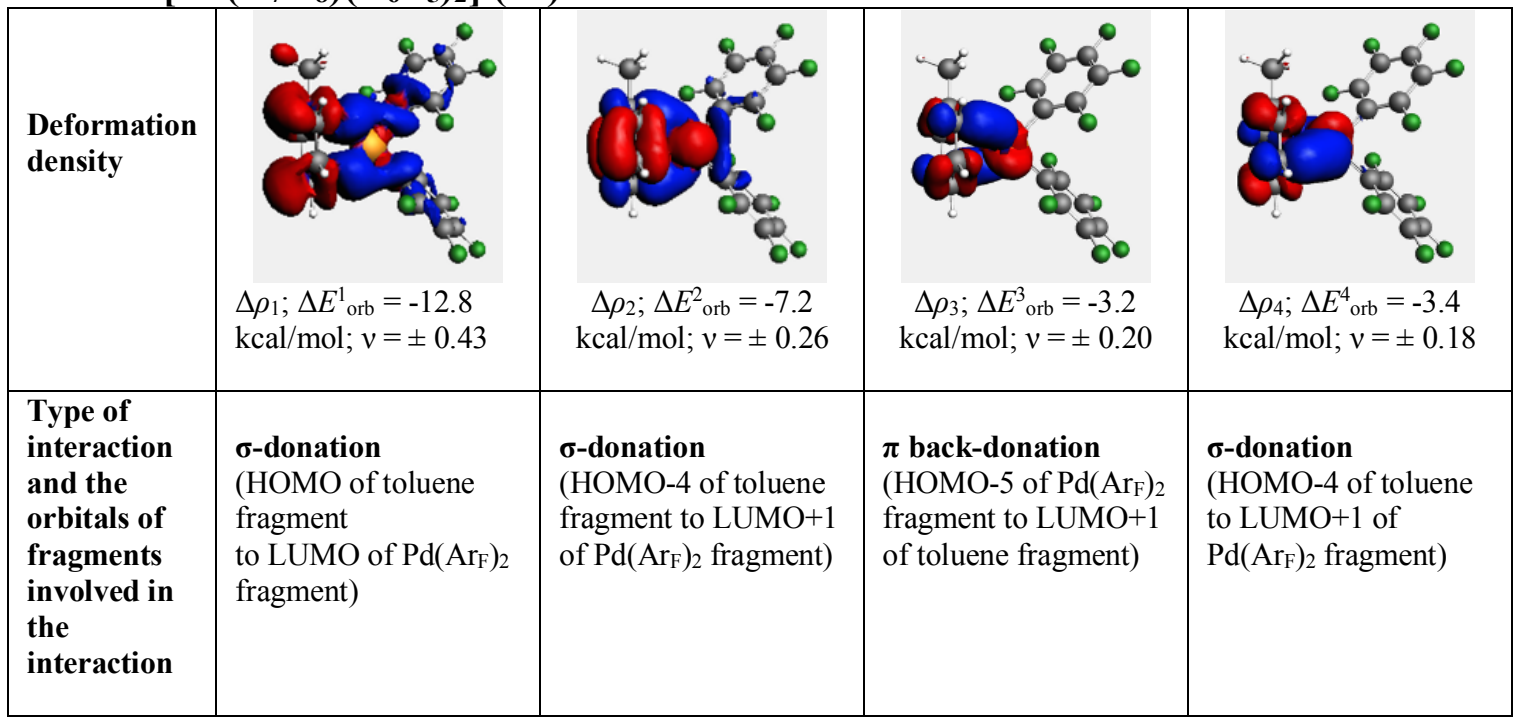

For TS(25-26):

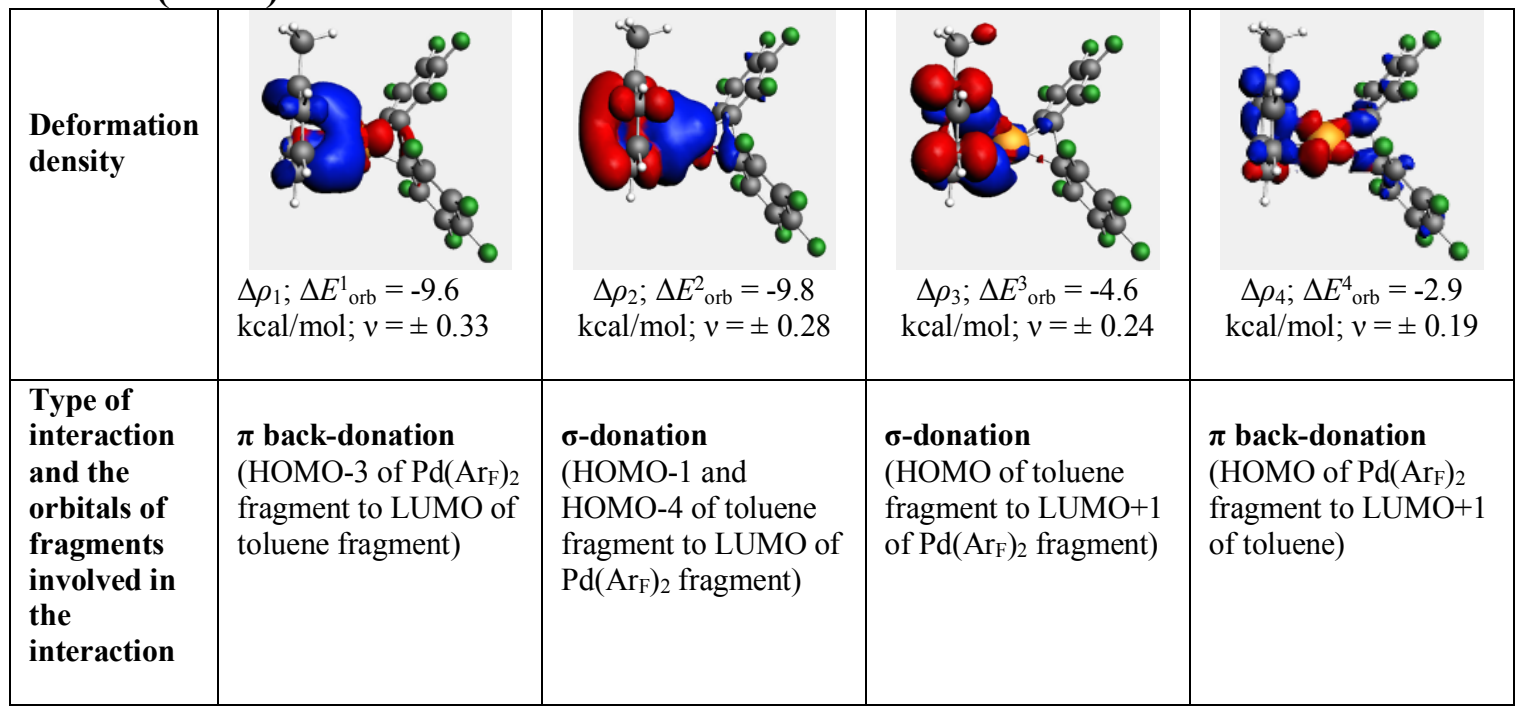


For cis- $\left[\mathrm{Pd}\left(\mathrm{C}_{8} \mathrm{H}_{10}\right)\left(\mathrm{C}_{6} \mathrm{~F}_{5}\right)_{2}\right](\mathbf{3 0})$ :

\begin{tabular}{|c|c|c|c|}
\hline $\begin{array}{l}\text { Deformation } \\
\text { density }\end{array}$ & $\begin{array}{l}\Delta \rho_{1} ; \Delta E_{\mathrm{orb}}^{1}=-13.4 \\
\mathrm{kcal} / \mathrm{mol} ; v= \pm 0.45\end{array}$ & $\begin{array}{c}\rho_{0} \\
\Delta \rho_{2} ; \Delta E_{\text {orb }}^{2}=-7.4 \\
\mathrm{kcal} / \mathrm{mol} ; v= \pm 0.26\end{array}$ & $\begin{array}{ccc}\Delta \rho_{3} ; \Delta E^{3} \text { orb }=-3.3 \\
\mathrm{kcal} / \mathrm{mol} ; v= \pm 0.19\end{array}$ \\
\hline $\begin{array}{l}\text { Type of } \\
\text { interaction } \\
\text { and the } \\
\text { orbitals of } \\
\text { fragments } \\
\text { involved in } \\
\text { the } \\
\text { interaction }\end{array}$ & $\begin{array}{l}\text { б-donation } \\
\text { (HOMO of } m \text {-xylene } \\
\text { fragment to LUMO of } \\
\left.\operatorname{Pd}\left(\mathrm{Ar}_{\mathrm{F}}\right)_{2} \text { fragment }\right)\end{array}$ & $\begin{array}{l}\text { б-donation } \\
\text { (HOMO- } 4 \text { of } m \text { - } \\
\text { xylene fragment to } \\
\text { LUMO+1 of } \mathrm{Pd}\left(\mathrm{Ar}_{\mathrm{F}}\right)_{2} \\
\text { fragment) }\end{array}$ & $\begin{array}{l}\boldsymbol{\pi} \text { back-donation } \\
\left(\mathrm{HOMO}-5 \text { of } \mathrm{Pd}\left(\mathrm{Ar}_{\mathrm{F}}\right)_{2}\right. \\
\text { fragment to } \mathrm{LUMO}+1 \\
\text { of } m \text {-xylene fragment) }\end{array}$ \\
\hline
\end{tabular}

For TS(30-31):

\begin{tabular}{|c|c|c|c|c|}
\hline $\begin{array}{l}\text { Deformation } \\
\text { density }\end{array}$ & i. & $0 \%$ कc & 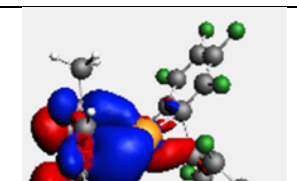 & ces \\
\hline & $\begin{array}{l}\Delta \rho_{1} ; \Delta E_{\text {orb }}^{1}=-8.6 \\
\mathrm{kcal} / \mathrm{mol} ; v= \pm 0.31\end{array}$ & $\begin{array}{c}\Delta \rho_{2} ; \Delta E^{2}{ }_{\text {orb }}=-9.7 \\
\mathrm{kcal} / \mathrm{mol} ; v= \pm 0.30\end{array}$ & $\begin{array}{c}\Delta \rho_{3} ; \Delta E_{\text {orb }}^{3}=-4.4 \\
\mathrm{kcal} / \mathrm{mol} ; v= \pm 0.22\end{array}$ & $\begin{array}{c}\Delta \rho_{4} ; \Delta E^{4}{ }_{\text {orb }}=-2.9 \\
\mathrm{kcal} / \mathrm{mol} ; v= \pm 0.19\end{array}$ \\
\hline $\begin{array}{l}\text { Type of } \\
\text { interaction } \\
\text { and the } \\
\text { orbitals of } \\
\text { fragments } \\
\text { involved in } \\
\text { the } \\
\text { interaction }\end{array}$ & $\begin{array}{l}\boldsymbol{\pi} \text { back-donation } \\
\left(\mathrm{HOMO}-3 \text { of } \mathrm{Pd}\left(\mathrm{Ar}_{\mathrm{F}}\right)_{2}\right. \\
\text { fragment to } \mathrm{LUMO}+1 \\
\text { of } m \text {-xylene fragment) }\end{array}$ & $\begin{array}{l}\text { б-donation } \\
\text { (HOMO of } m \text {-xylene } \\
\text { fragment to LUMO of } \\
\left.\operatorname{Pd}\left(\mathrm{Ar}_{\mathrm{F}}\right)_{2} \text { fragment }\right)\end{array}$ & $\begin{array}{l}\text { o-donation } \\
\text { (HOMO- } 1 \text { of } m \text { - } \\
\text { xylene fragment to } \\
\text { LUMO }+1 \text { of } \operatorname{Pd}\left(\mathrm{Ar}_{\mathrm{F}}\right)_{2} \\
\text { fragment) }\end{array}$ & $\begin{array}{l}\boldsymbol{\pi} \text { back-donation } \\
\left(\mathrm{HOMO} \text { of } \mathrm{Pd}\left(\mathrm{Ar}_{\mathrm{F}}\right)_{2}\right. \\
\text { fragment to } \mathrm{LUMO} \text { of } \\
m \text {-xylene })\end{array}$ \\
\hline
\end{tabular}


For cis- $\left[\mathrm{Pd}\left(\mathrm{C}_{6} \mathrm{~F}_{6}\right)\left(\mathrm{C}_{6} \mathrm{~F}_{5}\right)_{2}\right](\mathbf{3 5})$ :

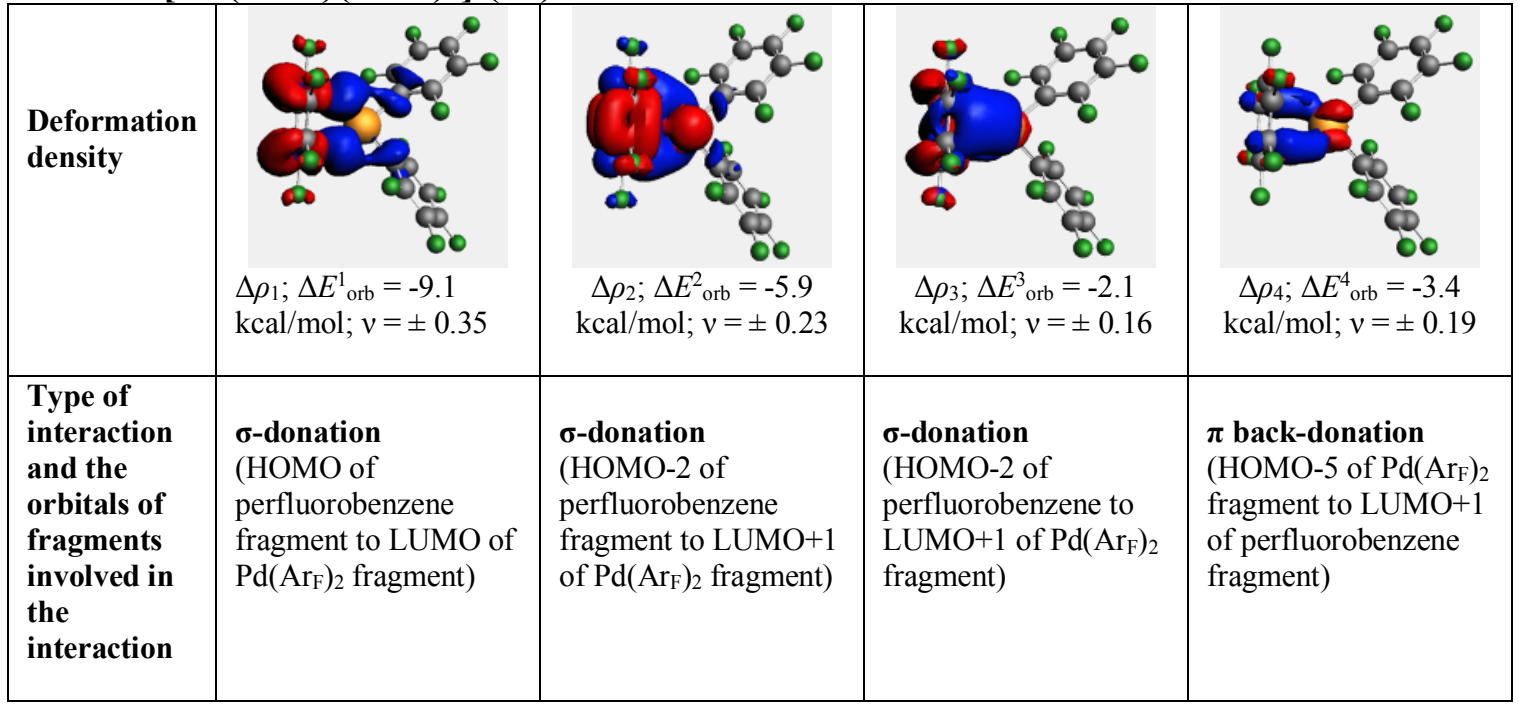

For TS(35-36):

\begin{tabular}{|c|c|c|c|c|}
\hline $\begin{array}{l}\text { Deformation } \\
\text { density }\end{array}$ & $\begin{array}{l}\qquad \rho_{1} ; \Delta E_{\mathrm{orb}}^{1}=-11.6 \\
\mathrm{kcal} / \mathrm{mol} ; \mathrm{v}= \pm 0.35\end{array}$ & 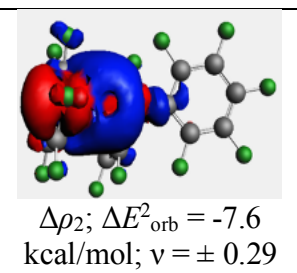 & $\begin{array}{l}\text { } \\
\begin{array}{l}\Delta \rho_{3} ; \Delta E^{3}{ }_{\mathrm{orb}}=-4.0 \\
\mathrm{kcal} / \mathrm{mol} ; \mathrm{v}= \pm 0.21\end{array}\end{array}$ & $\begin{array}{l}\text { } \\
\Delta \rho_{4} ; \Delta E_{\mathrm{orb}}^{4}=-2.0 \\
\mathrm{kcal} / \mathrm{mol} ; v= \pm 0.12\end{array}$ \\
\hline $\begin{array}{l}\text { Type of } \\
\text { interaction } \\
\text { and the } \\
\text { orbitals of } \\
\text { fragments } \\
\text { involved in } \\
\text { the } \\
\text { interaction }\end{array}$ & $\begin{array}{l}\boldsymbol{\pi} \text { back-donation } \\
\left(\mathrm{HOMO}-1 \text { of } \mathrm{Pd}\left(\mathrm{Ar}_{\mathrm{F}}\right)_{2}\right. \\
\text { fragment to LUMO of } \\
\text { perfluorobenzene } \\
\text { fragment) }\end{array}$ & $\begin{array}{l}\text { б-donation } \\
\text { (HOMO of } \\
\text { perfluorobenzene } \\
\text { fragment to LUMO of } \\
\operatorname{Pd}\left(\mathrm{Ar}_{\mathrm{F}}\right)_{2} \text { fragment) }\end{array}$ & $\begin{array}{l}\boldsymbol{\pi} \text { back-donation } \\
\left(\mathrm{HOMO}-6 \text { of } \mathrm{Pd}\left(\mathrm{Ar}_{\mathrm{F}}\right)_{2}\right. \\
\text { fragment to } \mathrm{LUMO}+1 \\
\text { of perfluorobenzene })\end{array}$ & $\begin{array}{l}\text { б-donation } \\
\text { (HOMO-2 of } \\
\text { perfluorobenzene } \\
\text { fragment to LUMO+1 } \\
\text { of } \operatorname{Pd}\left(\mathrm{Ar}_{\mathrm{F}}\right)_{2} \text { fragment) }\end{array}$ \\
\hline
\end{tabular}




\section{References}

(S1) Ishiyama, T.; Takagi, J.; Hartwig. J. F.; Miyaura, N. A stoichiometric aromatic C-H borylation catalyzed by iridium(I)2,2'-bypiridine complexes at room temperature. Angew. Chem., Int. Ed. 2002, 41, 3056-3058.

(S2) Tsoi, Y. T.; Zhou, Z.; Yu, W. Y. Rhodium-catalyzed cross-coupling reaction of arylboronates and diazoesters and tandem alkylation reaction for the synthesis of quaternary $\alpha, \alpha$-heterodiaryl carboxylic esters. Org. Lett. 2011, 13, 5370-5373.

(S3) Hofer, M.; Gomez-Bengoa, E.; Nevado, C. A neutral gold(III)-boron transmetalation. Organometallics 2014, 33, 1328-1332.

(S4) Deacon, G. B.; Phillips, R. J. Organothallium compounds. XIV. Thermal decomposition of some polyfluorobenzoatobis(polyfluorophenyl)thallium(III) compounds. Aust. J. Chem. 1978, 31, 1729-1724.

(S5) Kikushima, K.; Grellier, M.; Ohashi, M.; Ogoshi, M. Transition-metal-free catalytic hydrodefluorination of polyfluoroarenes by concerted nucleophilic aromatic substitution with a hydrosilicate. Angew. Chem., Int. Ed. 2017, 56, 16191-16196.

(S6) Truong, T.; Alvarado, J.; Tran, L. D.; Daugulis, O. Nickel, manganese, cobalt, and iron-catalyzed deprotonative arene dimerization. Org. Lett. 2010, 2, 1200-1203.

(S7) Deacon, G. B.; Koplick, A. J.; Ravertyand, W. D.; Vince, D. G. Organolanthanoids. II. Preparation and identification of some organolanthanoid species in solution. J. Org. Chem. 1979, 182, 121-141.

(S8) Maiti, B.; Wang, K.; Bhandari, S.; Bunge, S. D.; Twieg, R. J.; Dunietz, B. D. Enhancing charge mobilities in selectively fluorinated oligophenyl organic semiconductors: a design approach based on experimental and computational perspectives. J. Mater. Chem. C, 2019, 7, 3881-3888.

(S9) Mulla, S. A. R.; Chavan, S. S.; Pathan, M. Y.; Inamdar, S. M.; Shaikh, T. M. Y. Ligand-, base-, co-catalyst-free copper fluorapatite (CuFAP) as a versatile, ecofriendly, heterogeneous and reusable catalyst for an efficient homocoupling of arylboronic acid at ambient reaction conditions. $R S C A d v$. 2015, 5, 24675-24680. 
(S10) Macgregor, S. A.; Christopher Roe, D.; Marshall, W. J.; Bloch, K. M.; Bakhmutov, V. I.; Grushin, V. V. The F/Ph Rearrangement reaction of $\left[\left(\mathrm{Ph}_{3} \mathrm{P}\right)_{3} \mathrm{RhF}\right]$, the fluoride congener of Wilkinson's catalyst. J. Am. Chem. Soc. 2005, 127, 15304-15321.

(S11) Sheldrick, G. Acta Crystallogr. SHELXT - Integrated space-group and crystal-structure determination. 2015, $A 71,3-8$.

(S12) Sheldrick, G. Acta Crystallogr. A short history of SHELX. 2008, A64, 112-122.

(S13) Hübschle, C. B.; Sheldrick, G. M.; Dittrich, B. ShelXle: a Qt graphical user interface for SHELXL. J. Appl. Crystallogr. 2011, 44, 1281-1284.

(S14) Brandenburg, K. Diamond (version 4.4.0), Crystal and Molecular Structure Visualization, Crystal Impact H. Putz \& K. Brandenburg GbR, Bonn (Germany), 2017.

(S15) M. J. Frisch,; G. W. Trucks.; Schlegel, H. B.; Scuseria, G. E.; Robb, M. A.; Cheeseman, J. R.; Scalmani, G.; Barone, V.; Mennucci, B.; Petersson, G. A.; Nakatsuji, H.; Caricato, M.; Li, X.; Hratchian, H. P.; Izmaylov, A. F.; Bloino, J.; Zheng, G.; Sonnenberg, J. L.; Hada, M.; Ehara, M.; Toyota, K.; Fukuda, R.; Hasegawa, J.; Ishida, M.; Nakajima, T.; Honda, Y.; Kitao, O.; Nakai, H.; Vreven, T.; Montgomery, Jr. A.; Peralta, J. E.; Ogliaro, F.; Bearpark, M.; Heyd, J. J.; Brothers, E.; Kudin, K. N.; Staroverov, V. N.; Keith, T.; Kobayashi, R.; Normand, J.; Raghavachari, K.; Rendell, A.; Burant, J. C.; Iyengar, S. S.; Tomasi, J.; Cossi, M.; Rega, N.; Millam, J. M.; Klene, M.; Knox, J. E.; Cross, J. B.; Bakken, V.; Adamo, C.; Jaramillo, J.; Gomperts, R.; Stratmann, R. E.; Yazyev, O.; Austin, A. J.; Cammi, R.; Pomelli, C.; Ochterski, J. W.; Martin, R. L.; Morokuma, K.; Zakrzewski, V. G.; Voth, G. A.; Salvador, P.; Dannenberg, J. J.; Dapprich, S.; Daniels, A. D.; Farkas, O.; Foresman, J. B.; Ortiz, J. V.; Cioslowski, J.; Fox, D. J. Gaussian 09 (Rev. E.01), Gaussian, Inc.: Wallingford CT, 2010.

(S16)(a) Becke, A. D. Density-functional exchange-energy approximation with correct asymptotic behavior. Phys. Rev. A, 1988, 38, 3098-3100. (b) Becke, A. D. Densityfunctional thermochemistry. III. The role of exact exchange. J. Chem. Phys. 1993, 98, 5648-5652. (c) Lee, C.; Yang, W.; Parr, R. G. Development of the Colle-Salvetti correlation-energy formula into a functional of the electron density. Phys. Rev. B, 1988, 37, 785-789. 
(S17) Tomasi, J.; Mennucci, B.; Cammi, R. Quantum mechanical continuum solvation models, Chem. Rev. 2005, 105, 2999-3094.

(S18) (a) Grimme, S.; Antony, S.; Ehrlich, S.; Krieg, H. A consistent and accurate ab initio parameterization of density functional dispersion correction (DFT-D) for the 94 elements H-Pu. J. Chem. Phys., 2010, 132, 15414. (b) Grimme, S.; Ehrlich, S.; Goerigk, L. Effect of the damping function in dispersion corrected density functional theory. J. Comp. Chem. 2011, 32, 1456-1465.

(S19) Baker, J. An algorithm for the location of transition states. J. Comp. Chem. 1986, 7, 385-395.

(S20) (a) East, A. L. L.; Berner, G. M.; Morcom, A. D.; Mihichuk, L. Computational study of tungsten(II)-catalyzed rearrangements of norbornadiene. J. Chem. Theory Comput., 2008, 4, 1274-1282. (b) Jayaraman, A. A.; East, L. L. The mechanism of permanganate oxidation of sulfides and sulfoxides. J. Org. Chem. 2012, 77, 351-356. (c) Nilewar, S.; Jayaraman, A.; Sterenberg, B. T. Alkyne insertion into $\mathrm{P}-\mathrm{C}(\mathrm{sp} 2)$ bonds as a route to fused phospholes: transition-metal-like reactivity at phosphorus. Organometallics 2018, 37, 4699-4710. (d) Jayaraman, A.; Berner, G. M.; Mihichuk, L. M.; East, A. L. L. Tungsten(II)-catalyzed rearrangements of norbornadiene: Effects of alternative complexation stages. J. Mol. Catal. A. 2011, 351, 143-153. (e) Jayaraman, A.; PowellDavies, H.; Fontaine, F.-G. Revisiting the reduction of indoles by hydroboranes: A combined experimental and computational study. Tetrahedron 2019, 75, 2118-2127.

(S21) ADF 2017.207, SCM, Theoretical chemistry, Vrije Universiteit, Amsterdam, The Netherlands, http://www.scm.com.

(S22) (a) Morokuma, K. J. Molecular orbital studies of hydrogen bonds. III. C=O $\cdots \mathrm{H}-\mathrm{O}$ Hydrogen Bond in $\mathrm{H}_{2} \mathrm{CO} \cdots \mathrm{H}_{2} \mathrm{O}$ and $\mathrm{H}_{2} \mathrm{CO} \cdots 2 \mathrm{H}_{2} \mathrm{O}$. Chem. Phys. 1971, 55, 1236. (b) Ziegler, T.; Rauk, A. CO, CS, $\mathrm{N}_{2}, \mathrm{PF}_{3}$, and $\mathrm{CNCH}_{3}$ as $\sigma$ Donors and $\pi$ acceptors. A theoretical study by the Hartree-Fock-Slater transition-state method. Inorg. Chem. 1979, 18, 1755-1759.

(S23) (a) Mitoraj, M.; Michalak, A. Donor-acceptor properties of ligands from the natural orbitals for chemical valence. Organometallics 2007, 26, 6576-6580. (b) Mitoraj, M.; Michalak, A. Applications of natural orbitals for chemical valence in a description of bonding in conjugated molecules. J. Mol. Model 2008, 14, 681-687. 
(S24) Mitoraj. M. P.; Michalak, A.; Ziegler, T. A combined charge and energy decomposition scheme for bond analysis. J. Chem. Theory Comput. 2009, 5, 962-975.

(S25) (a) Zhao, L.; von Hopffgarten, M.; Andrada, D. M.; Frenking, G. Energy decomposition analysis. Wiley Interdiscip. Rev. Comput. Mol. Sci. 2018, 8, e1345. (b) Bickelhaupt, F. M.; Baerends, E. J. In Kohn-Sham Density Functional Theory: Predicting and Understanding Chemistry; Lipkowitz, K. B., Boyd, D. B., Eds.; Reviews in Computational Chemistry Volume 15; Wiley-VCH: New York, 2000; p 186. 